\title{
Development of a constrained piston method for compression testing of carbon foams
}

\author{
Stephen P. Carpenter \\ West Virginia University
}

Follow this and additional works at: https://researchrepository.wvu.edu/etd

\section{Recommended Citation}

Carpenter, Stephen P., "Development of a constrained piston method for compression testing of carbon foams" (2005). Graduate Theses, Dissertations, and Problem Reports. 1585.

https://researchrepository.wvu.edu/etd/1585

This Thesis is protected by copyright and/or related rights. It has been brought to you by the The Research Repository @ WVU with permission from the rights-holder(s). You are free to use this Thesis in any way that is permitted by the copyright and related rights legislation that applies to your use. For other uses you must obtain permission from the rights-holder(s) directly, unless additional rights are indicated by a Creative Commons license in the record and/ or on the work itself. This Thesis has been accepted for inclusion in WVU Graduate Theses, Dissertations, and Problem Reports collection by an authorized administrator of The Research Repository @ WVU. For more information, please contact researchrepository@mail.wvu.edu. 


\title{
Development of a Constrained Piston Method for Compression Testing of Carbon Foams
}

\author{
Stephen P. Carpenter \\ Thesis submitted to the \\ College of Engineering and Mineral Resources \\ at West Virginia University \\ in partial fulfillment of the requirements for the degree of \\ Master of Science \\ in \\ Chemical Engineering
}

John W. Zondlo, Ph.D., Chair

Peter G. Stansberry, Ph.D.

Liviu Magean, M.S.

Department of Chemical Engineering

Morgantown, WV

2005

Keywords: Carbon Foam, Compression Test, Compressive Strength

Copyright 2005 Stephen P. Carpenter 


\begin{abstract}
Development of a Constrained Piston Method for Compression Testing of Carbon Foams
\end{abstract}

\author{
Stephen P. Carpenter
}

The purpose of this study is to develop a standard compression test for gathering mechanical strength properties, namely yield stress, modulus, and energy absorption all during a single compression test. Several carbon foams were tested using both the novel constrained method (developed herein) and the conventional unconstrained technique to compare the results. Statistical analysis has been performed on compression tests from three different carbon foams. Several other foams have also been tested to demonstrate that this procedure can be performed on any type of carbon foam, regardless of its precursor material or manufacturing process. This study shows that the constrained test gives nearly identical values of both yield stress and modulus compared to those from the unconstrained test. Yield stress results have been gathered at a 95 percent confidence interval with less than 1.5 percent error, and modulus values at less than 4 percent error. In addition, energy absorption results have been successfully gathered using the same test. 


\section{Contents}

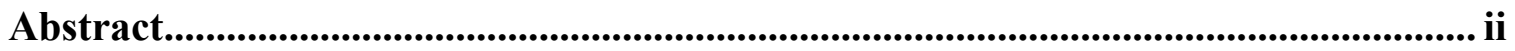

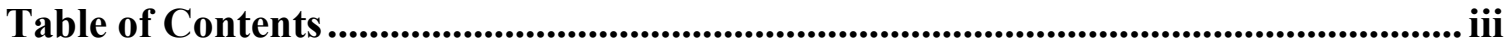

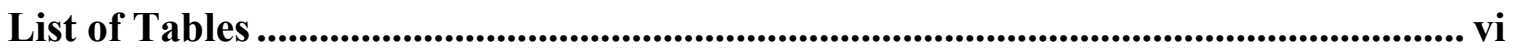

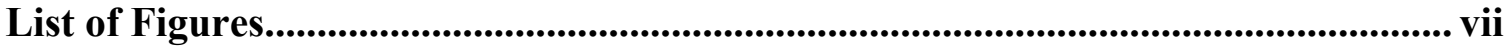

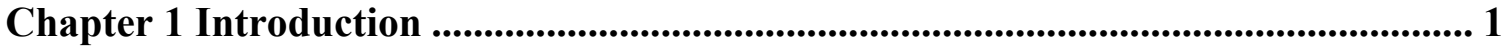

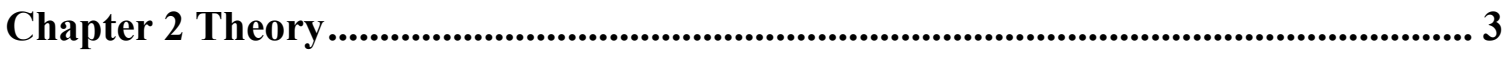

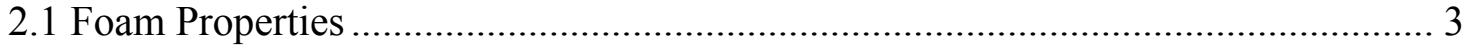

2.2 Foam and Cell-Wall Properties................................................................. 4

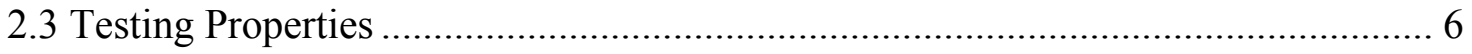

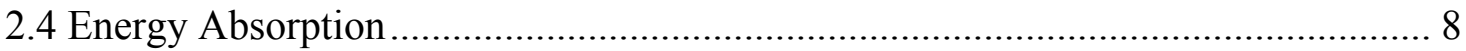

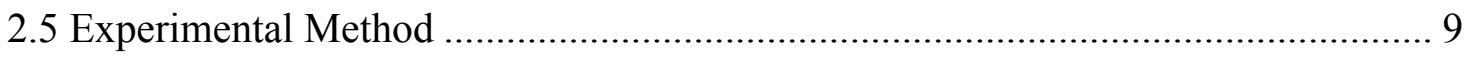

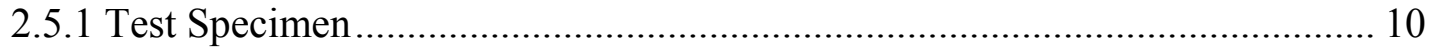

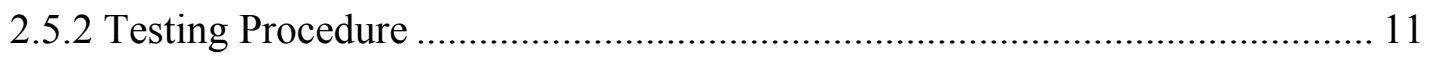

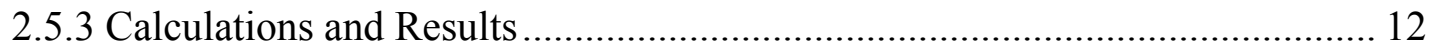

Chapter 3 Experimental Method................................................................................................. 14

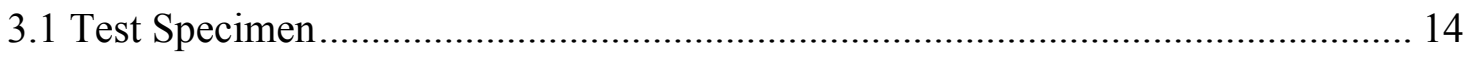

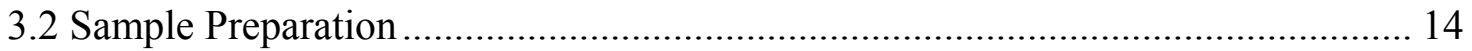

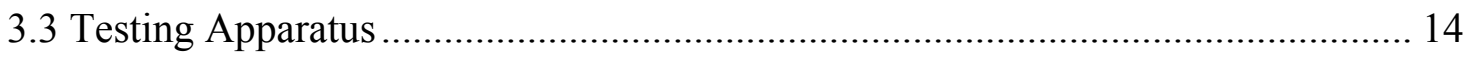

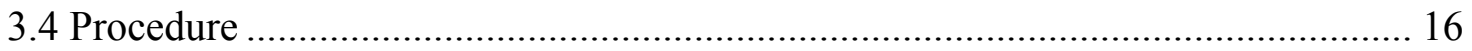

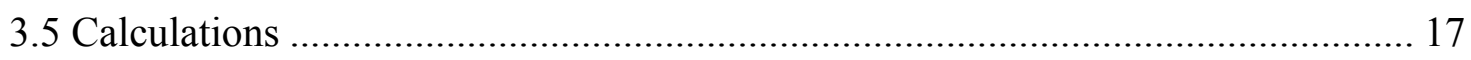

Chapter 4 Preliminary Results ................................................................................................ 19

Chapter 5 Project Objectives....................................................................................... 24 


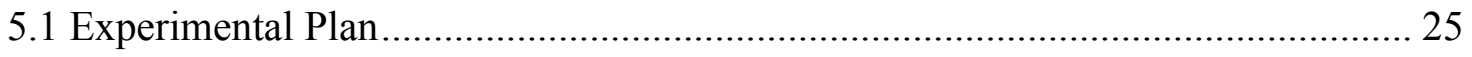

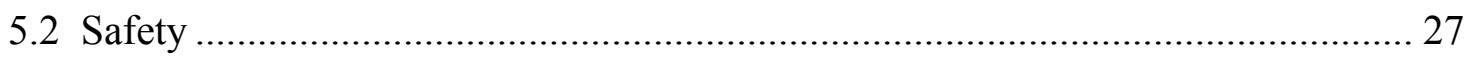

Chapter 6 Results and Discussion ...................................................................................... 29

6.1 GrafTech Foam Results ................................................................................. 30

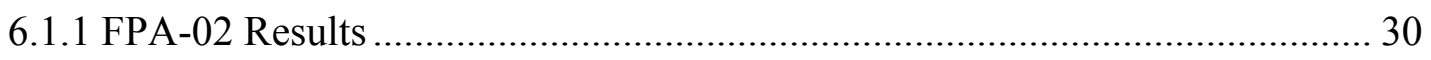

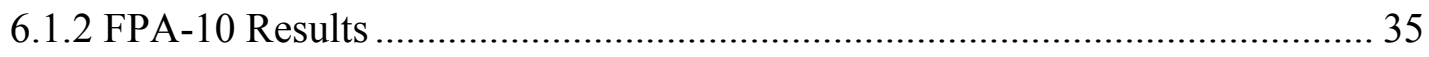

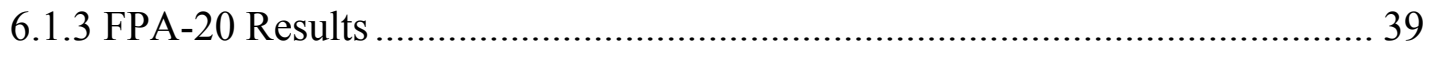

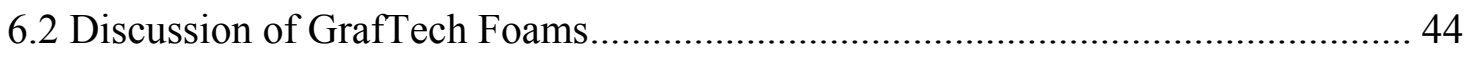

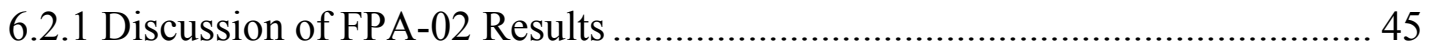

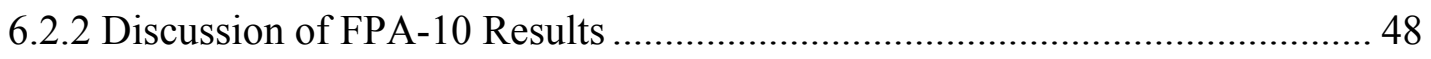

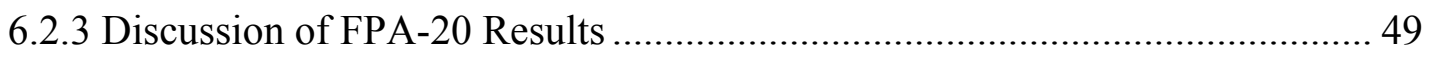

6.3 MER, WVU, and Ultramet Foam Results …………………………………..... 51

6.3.1 MER Foam Results ................................................................................... 52

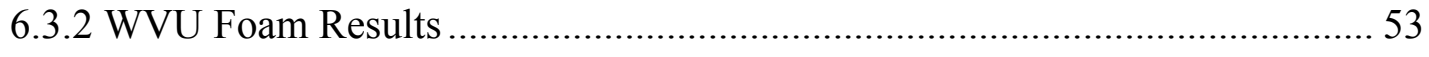

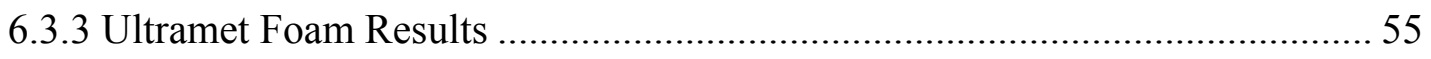

6.4 Discussion of MER, WVU, and Ultramet Foam Results ..................................... 56

6.4.1 Discussion of MER Foam Results …………………................................ 57

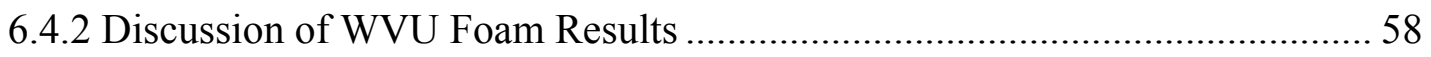

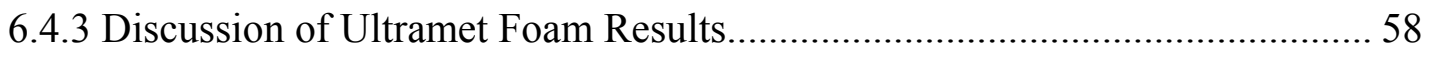

6.5 Observations from Results............................................................................ 59

Chapter 7 Summary and Conclusions ............................................................................. 63

Chapter 8 Recommendations.................................................................................................. 65

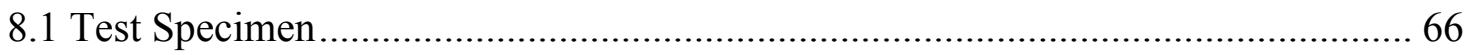




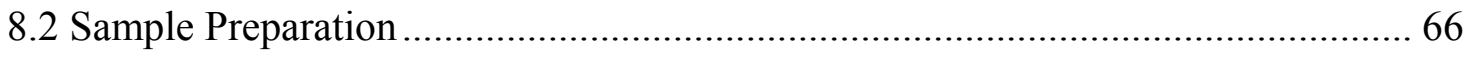

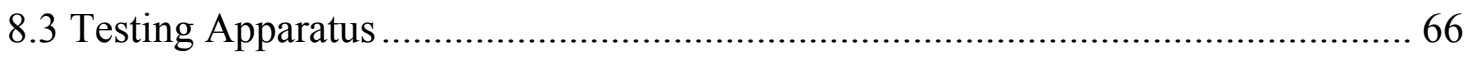

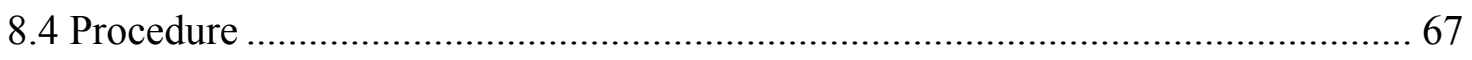

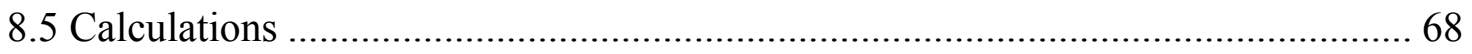

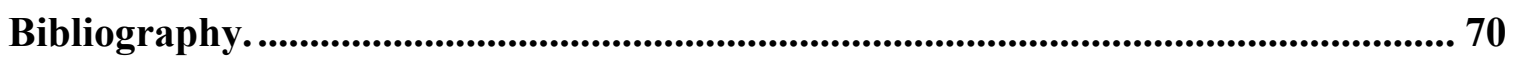

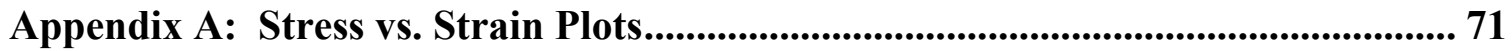

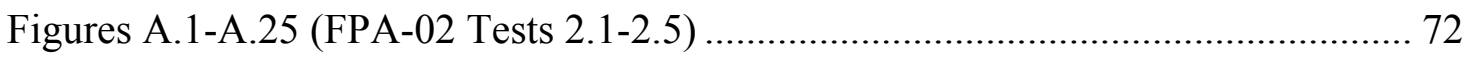

Figures A.26-A.40 (FPA-10 Tests 10.1-10.3) ….................................................. 97

Figures A.41-A.55 (FPA-20 Tests 20.1-20.3) .................................................... 112

Figures A.56-A.58 (MER Tests MER.1-MER.2) …........................................... 127

Figures A.59-A.63 (WVU Tests WVU.1-WVU.2) .............................................. 130

Figures A.64-A.72 (Ultramet Tests U.1-U.4) .................................................... 135 


\section{List of Tables}

Table 4.1 Results from First USAF Round Robin Test .............................................. 21

Table 4.2 Results from Second USAF Round Robin Test ............................................ 22

Table 6.1 Parameters for FPA-02 Compression Tests................................................. 31

Table 6.2 Mean Results for FPA-02 Carbon Foam ………………………….............. 33

Table 6.3 Confidence Intervals for FPA-02 Data ......................................................... 33

Table 6.4 Parameters for FPA-10 Compression Tests................................................. 37

Table 6.5 Mean Results for FPA-10 Carbon Foam …………..................................... 37

Table 6.6 Confidence Intervals for FPA-10 Data ........................................................ 39

Table 6.7 Parameters for FPA-20 Compression Tests.................................................. 41

Table 6.8 Mean Results for FPA-20 Carbon Foam ....................................................... 42

Table 6.9 Confidence Intervals for FPA-20 Data ..................................................... 42

Table 6.10 Confidence Intervals for Test 20.3 of FPA-20 ………………………........ 43

Table 6.11 Complete Results for Test 2.5 of FPA-02 ………………………............ 47

Table 6.12 Parameters for MER Foam Compression Tests............................................. 53

Table 6.13 Mean Results for MER Carbon Foam ………………………….............. 53

Table 6.14 Parameters for WVU Foam Compression Tests............................................ 54

Table 6.15 Mean Results for WVU Carbon Foam …………………………............... 54

Table 6.16 Parameters for Ultramet Foam Compression Tests ...................................... 56

Table 6.17 Mean Results for Ultramet Carbon Foam.................................................... 56 


\section{List of Figures}

Figure 2.1 Typical Stress vs. Strain Plot for Foam .................................................... 4

Figure 2.2 SEM Micrograph of Anisotropic Cell Structure of a Carbon Foam ............... 6

Figure 3.1 Photographs of Piston Assembly........................................................... 15

Figure 3.2 Schematic of Piston Assembly with Stress vs. Strain Plot........................... 16

Figure 4.1 Plot of Incomplete Stress vs. Strain Data of an Ultramet Sample................. 23

Figure 6.1 Photo and SEM Micrograph of FPA-02 Carbon Foam ............................... 31

Figure 6.2 Representative Histograms of Yield Stress and Energy Absorption.............. 32

Figure 6.3 Photos of Unconstrained Compression of FPA-02 Carbon Foam ................ 34

Figure 6.4 Photo and SEM Micrograph of FPA-10 Carbon Foam ............................... 36

Figure 6.5 Photos of Unconstrained Compression of FPA-10 Carbon Foam ................ 38

Figure 6.6 Photo and SEM Micrograph of FPA-20 Carbon Foam ............................... 40

Figure 6.7 Photos of Unconstrained Compression of FPA-20 Carbon Foam ................ 43

Figure 6.8 Photos of Constrained Compression of FPA-20 Carbon Foam .................... 44

Figure 6.9 SEM Micrograph of Carbon Foam from MER Corporation ......................... 52

Figure 6.10 SEM Micrograph of Carbon Foam from WVU .................................... 54

Figure 6.11 SEM Micrograph of Carbon Foam from Ultramet.................................... 55 


\section{Chapter 1}

\section{Introduction}

Man-made foams, manufactured on a large scale, are used for absorbing the energy of impacts and in lightweight structures. Their efficient use requires a detailed understanding of their mechanical behavior. Such is the case with the development of carbon foam. Carbon foam is a relatively new product, still being researched in all aspects. Researchers are searching for the most efficient uses of this product, as well as better ways to measure and understand it properties.

Some of the most sought after characteristics of carbon foams are their mechanical properties: yield strength, Young's Modulus, and energy absorption. These particular properties are essential to determine specific uses for different types of carbon foams. However, different carbon foam manufacturers are using different analytical techniques to obtain these properties. Compression and tensile testing are both done for stress analysis, different samples are prepared in different ways, and different instruments are used by different labs to test these mechanical properties on vastly different carbon foams. These variances in compression testing result in inconsistent properties reported by carbon foam manufacturers.

The purpose of this project is to develop a standard compression test that yields consistent results for different carbon foams. A standard test should be able to gather yield strength, Young's modulus, and energy absorption data on any type of carbon foam. It should be applicable to both low-density as well as high-density foams. This standard test should also be able to reproduce very reliable results for any lab that tests the same 
type of foam created at the same conditions[1]. The specific tasks proposed for the present research are as follows:

- develop the compression test protocol and a suitable, easily reproduced piston assembly

- gather carbon foams of different densities from different manufacturers

- prepare all compression samples for compression testing

- visually compare microstructures of different carbon foams using SEM micrographs

- test carbon foam samples using both the unconstrained and constrained methods

- observe compression of foam samples through an optically transparent Lexan ${ }^{\circledR}$ cylinder

- compare data from all tests for each sample including yield stress, modulus, and energy absorption (if applicable) and perform statistical analysis of the data 


\section{Chapter 2}

\section{Theory}

Several items must be addressed before the mechanical properties of carbon foam can be evaluated. The most important issue is to determine which properties can be measured and what data can be observed as a result of a particular test. Other issues include studying the influences from differences in the materials being tested, sample preparation, and experimental parameters of the test itself.

\subsection{Foam properties}

The crucial foam properties to be measured are bulk density, the Young's Modulus, the yield strength, and fracture strength. Young's Modulus is the initial slope of the stress-strain compression curve that is linear (see Figure 2.1). The yield strength corresponds to the plateau of the stress-strain curve during compressive loading and is associated with the collapse of the cells. This collapse can occur by elastic buckling (as in elastomeric foam such as rubber), by the formation of plastic hinges in a foam that yield (such as a metallic foam), and by brittle crushing of cells within a brittle foam (such as carbon foam). The stress at which the compressive load reaches a point where the cell walls begin to physically break apart and fracture is called the fracture strength. The fracture strength and the yield strength are basically the same for brittle foams in which the cell walls do not bend much, but instead fracture under a compressive load. When the cells have almost completely collapsed, opposing cell walls touch and further strain compresses the solid itself. This gives a final region at the end of the stress-strain plateau 
of rapidly increasing stress known as the densification region. The energy required to compress the foam and collapse the cell walls can be determined by integrating the area under the stress-strain plot. Energy absorption is discussed in further detail in Section 2.4. These properties and areas of an actual stress-strain plot can be seen in Figure 2.1.

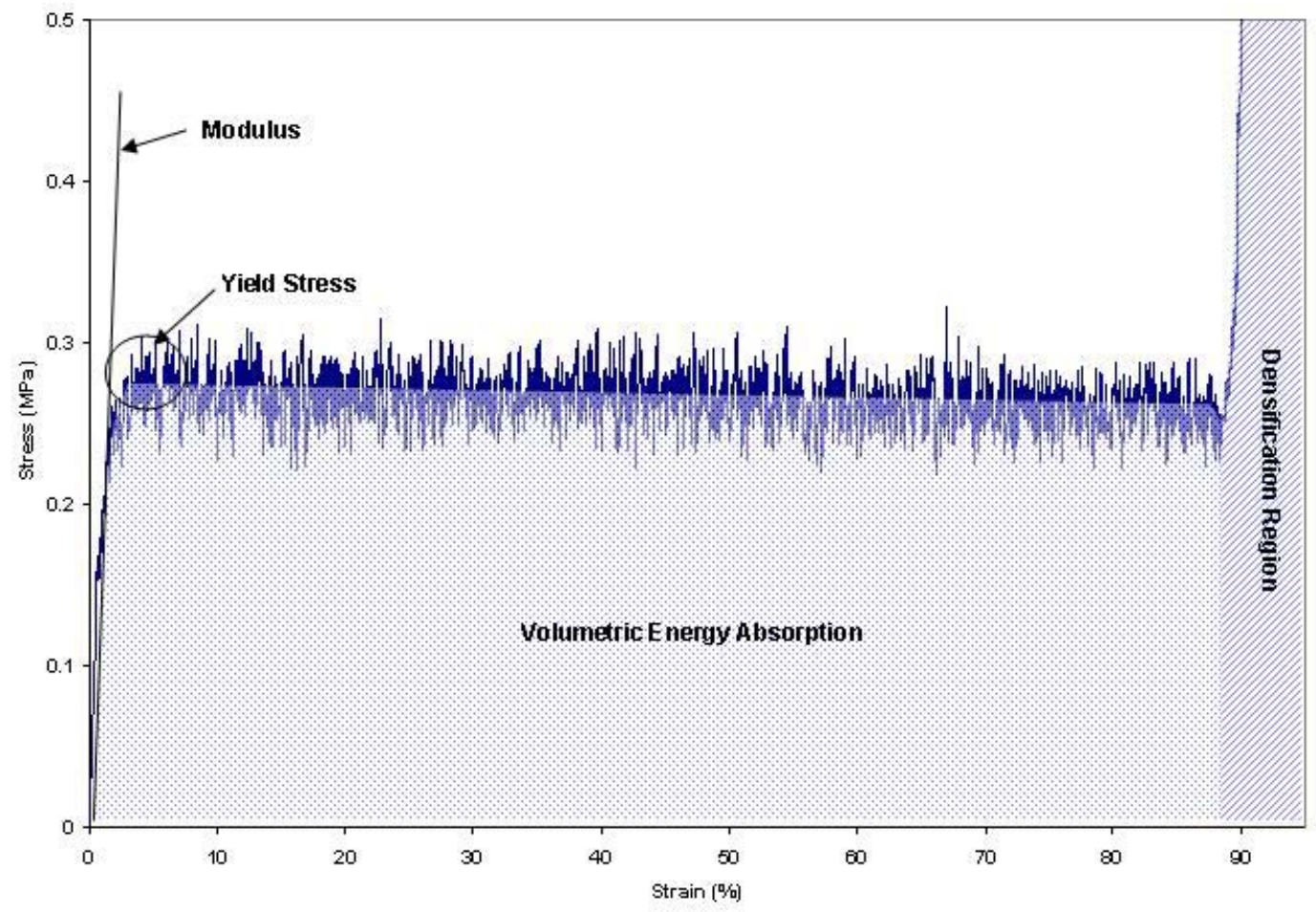

Figure 2.1. Typical Stress vs. Strain plot showing compressive behavior for a foam sample.

\subsection{Foam and Cell Wall Properties}

The most important structural features of any foam that can impact its mechanical properties are its relative density, the degree to which the cells are opened or closed, and 
the cells' shape anisotropy within the foam. The relative density is defined as the ratio of the bulk density of the foam divided by the density of the solid from which the foam was made. The higher a foam's relative density, the stronger the foam will be due to an increase of solid material within the foam matrix. Closed cell foams are generally stronger than open cell foams because of the increase of surface area of solid material surrounding each cell that must be fractured. Instead of having cells partially surrounded by solid material as is the case in open cells, the closed cells are completely encased in solid carbon material. However, surface tension usually forces the bulk of the material to accumulate in the outer edges or corners of the cells themselves, leaving little more than a thin membrane of solid material that closes the cell structures.

Cell anisotropy also has a large effect on foam strength. Most foams are anisotropic. Polymer foams, made by pouring the polymer plus a hardener and a foaming agent into an open mold, usually have cells that are elongated in the rise direction. Anisotropy can arise in two different ways. The more obvious is structural anisotropy. This is a directional dependence of the foam properties directly attributed to the shape of the cells, as in the open mold foaming mentioned. This directional cell shape can be seen in Figure 2.2. The other arises from material anisotropy in the properties of the cell walls. Anisotropy in the modulus, strength, and toughness of foams is a common observation. In polymer foams, there is a general agreement that the Young's Modulus is greatest in the rise direction; that is, the direction of greatest elongation of the cells, and an agreement that the plastic collapse strength of a rigid plastic foam, too, is greatest in this direction. However, despite the frequent reports of anisotropy, there are very few which 
contain enough information on cell shape and on properties in the three principle directions to allow a critical comparison with the theory[2].

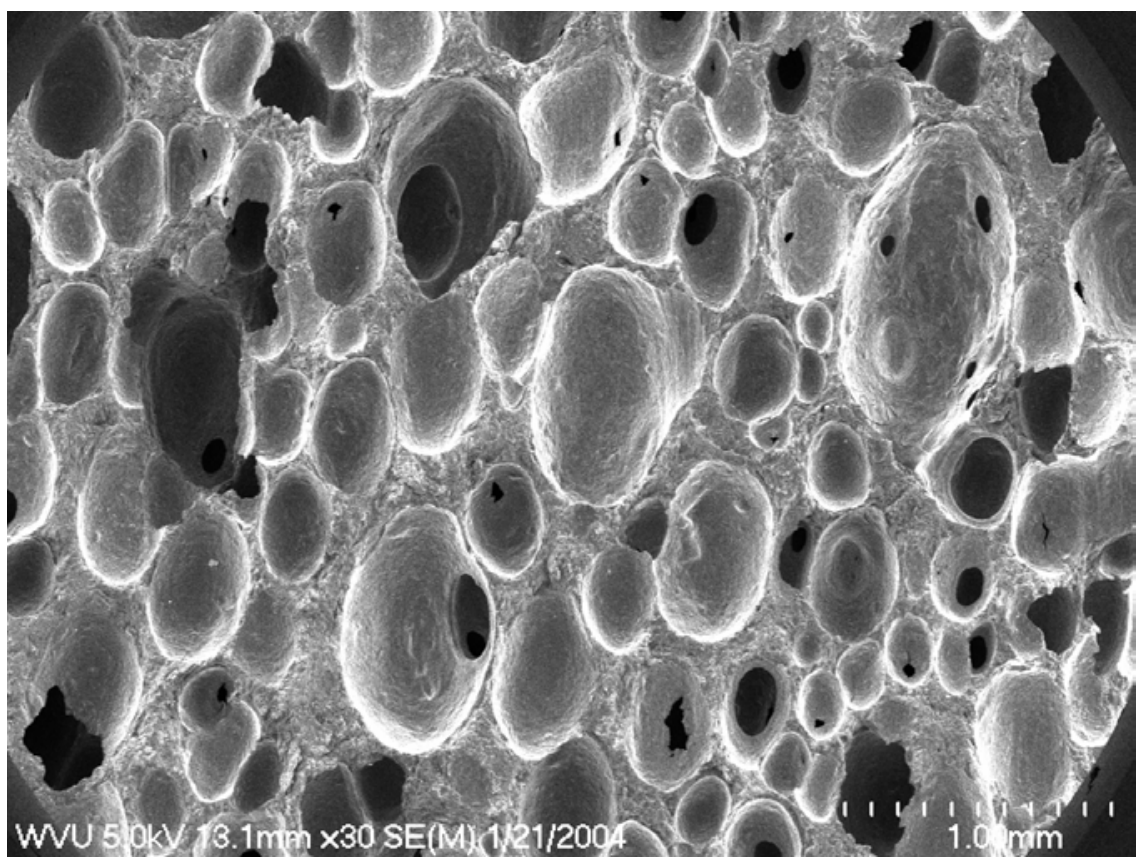

Figure 2.2. SEM micrograph of anisotropic cell structure of a carbon foam. (Foam sample courtesy of MER Corporation)

\subsection{Testing Properties}

There are a few controllable variables that have an impact on the results of a compression test. Temperature of the sample can be a major factor, especially when working with thermoplastic polymer-based foams. As the temperature rises and approaches the softening point of the material, the yield strength decreases due to the inherent softening of the material. However, for the case of carbon foams, this is not an issue since these foams are usually calcined to over $1000{ }^{\circ} \mathrm{C}$ and hence can withstand very high temperatures without softening. 
The ratio of the cross-sectional areas of the compressive device and the foam sample being compressed can also have an impact on test results for brittle foams such as carbon foam. Indentation tests of brittle foams with open cells, in which the compressive device has a smaller cross-section than the foam sample, show a surprising effect. The indentation pressure is not always equal to the compressive strength, but is strongly dependant on the size of the indenter face. It has been discovered that the maximum indentation pressure $P_{i}$ is inversely proportional to the square root of facial area of the indenter $A_{s}$, such that

$$
P_{i} \propto \frac{1}{\sqrt{A_{s}}}
$$

This size effect is valid for values of $A_{s}$ which are much greater than the area of a single cell[3]. Therefore, as the area of the compressive device gets smaller (compared to the area of the sample), the resultant compressive strength reading gets higher. However, if all the cell edges make contact with the indenter surface, the true yield stress can be determined from the test. That is to say, if the facial area of the indenter is equal to the apparent cross-sectional area of the foam sample, the resultant compressive strength reading is equal to the actual yield strength of the foam.

The strain rate of the compression test can influence the results as well. At high strain rates, inertial effects drive the compression strength upwards. There are three features of dynamic crushing that influence the overall force displacement response. Localization is the concentration of deformation at a given instant into a thin layer, often adjacent to the loading force. Caused by geometric softening, localization leads to local strain rates which are much larger than the apparent nominal strain-rate. Micro-inertia is associated with rotation and lateral motion of cell walls when they buckle. This tends to 
suppress the more compliant buckling modes, thus increasing the crushing stress. Densification causes the stress to rise steeply when cell walls come into contact. This leads to "shock" enhancement at very high strain rates because the cell collapse is overdriven during densification. These three phenomena will inflate the real results of yield strength for brittle foams thus causing increased energy absorption behavior.

\subsection{Energy Absorption}

Foams are especially good for energy adsorption. Compared to the solid from which it is made, the foam always generates a lower peak force for the same energy absorption. Energy is absorbed as cell walls bend plastically, buckle, or fracture, but the stress is limited by the long, flat plateau of the stress-strain curve.

When the foam is loaded, work is done by the forces applied to it. The work per unit volume in deforming the foam to a strain $E$ is simply the area under the stress-strain curve up to the strain $E$. Very little energy is absorbed initially in the short, linear-elastic portion of the curve. It is the long plateau of the curve, arising from cell collapse by buckling, yielding, or crushing, which allows large energy absorption at near-constant load. The level of this plateau depends on the material and density of the foam, and on the rate of strain. Ordinary laboratory compressive testing equipment provide low strain rates, $10^{-8}$ to $10^{-2} / \mathrm{s}$. Converted to an impact velocity on a compressible foam $100 \mathrm{~mm}$ thick is $10^{-9}$ to $10^{-3} \mathrm{~m} / \mathrm{s}$. Free fall from a height of 1 meter gives an impact-velocity of just over $4 \mathrm{~m} / \mathrm{s}$, with associated strain rates (same $100 \mathrm{~mm}$ thickness) of around $40 / \mathrm{s}$. These intermediate strain rates are reproduced in the laboratory with high-speed servo-hydraulic 
testing equipment and drop hammer tests. Automotive applications require protection against impacts up to $40 / \mathrm{s}$ and ballistic impacts can be 50 times faster than that, corresponding to strain rates up to $10^{4} / \mathrm{s}$. Dynamic loading devices, gas guns, and explosive loading equipment allow this high strain rate to be investigated. The upper end of this range lies above the sound velocity in all foams, and here inertial effects drive the plateau levels upward rapidly. However, dynamic loading of foams is rarely relevant for the kind of packaging applications in which foams excel[3].

\subsection{Experimental Methods}

In order to find the best testing method for compressing carbon foam, ASTM standard testing methods were researched for similar tests on similar materials. Five ASTM testing methods were referenced for sample preparation, testing apparatus and protocol, and for data acquisition and processing.

C 67-99a Standard Test Methods for Sampling and Testing Brick and Structural Clay Tile [4]

C 695-91(95) Standard Test Method for Compressive Strength of Carbon and Graphite $[5]$

D 1621-00 Standard Test Method for Compressive Properties of Rigid Cellular Plastics [6]

D 696-96 Standard Test Method for Compressive Properties of Rigid Plastics [7]

F 414-96 Standard Test Method for Energy Absorbed by a Tire When Deformed by a Slow-Moving Plunger [8] 
The first four methods deal directly with measuring the yield strength of the material. Each test is stopped when the yield stress has been reached and the sample being tested has failed. The final test (F 414-96) measures only the energy absorbed by a tire from a plunger that is forced into its treads.

\subsubsection{Test Specimen}

The four compressive testing methods each have very small variations in test specimen requirements. However, all agree that at least five specimens must be tested to yield reliable, statistical results.

All tests request a right-angled volume with a circular cross-section. Some of the tests leave the option for a square cross-section, but all tests mention a right cylinder. The measurements of each specimen depend on particle sizes or cell sizes within each material being tested. For example, the carbon and graphite test requests a diameter greater than ten times the size of the largest particle. The rigid cellular plastic foam test,

on the other hand, specifies a cross-section between $4-36 \mathrm{in}^{2}$. The specimen height requirement of each test varies slightly as well. Carbon and graphite characterization requires a height-to-diameter ratio between 1.9-2.1 to 1.0. The cellular plastic foam test specifies a minimum height of 1 inch and a maximum of no greater than the specimen diameter.

All tests also require some conditioning of the samples before they are tested. These instructions vary, but all agree that the specimens must be dry and well 
conditioned to the humidity and temperature of the room in which the specimen will be tested.

\subsubsection{Testing Procedure}

All ASTM methods described here dictate similar instructions. Specimen dimensions are to be recorded as an average of three measurements for each diameter and height by a caliper to \pm 0.001 in. Measurements should be taken when specimen and caliper faces come in contact. The caliper should not compress the specimen for any measurements. The specimen should be centered in the testing apparatus within $5 \%$ of the specimen diameter.

Each test describes platen movement differently, according to the requirements and expectations of each material being tested. Brick and clay tile testing defines the platen speed by applying half of the expected maximum load at any rate and the remainder of the load must be applied within 1-2 minutes. The carbon and graphite test requires continuous load to be applied so that specimen rupture time is greater than 30 seconds. Rigid cellular plastic foam testing specifies platen movement at $2.5 \pm 0.25$ $\mathrm{mm} / \mathrm{min}$ and the solid rigid plastic test specifies $1.3 \pm 0.3 \mathrm{~mm} / \mathrm{min}$. Finally, the energy absorption test for a tire requires a plunger speed of $50 \mathrm{~mm} / \mathrm{min}$.

The tests described above are all of an unconstrained method. That is to say that the sample being compressed is not contained within or constrained by any physical apparatus or sample holder. In the case of very dense foams, this usually results in multi- 
directional volume change. When failure occurs at the yield stress, the sample usually breaks apart causing the cross-section to change as pieces of foam break off. Most carbon foams are currently tested using some type of unconstrained method to gather modulus and yield stress. When failure occurs, the test is ended. However, the total energy absorption cannot be assessed by these techniques. Therefore, an alternate procedure must be developed so that the entire stress/strain curve (as shown in Figure 2.1) can be measured. The development of this new test is the focus of the present research proposal. Details of the test and the apparatus are described below.

\subsubsection{Calculations and Results}

Each of the current ASTM tests record the same information with respect to gathering and calculating the desired results. Compressive strength (also yield strength in the case of brittle foams) $C$ is defined as the load $F_{f}$ at the time of sample failure divided by the cross-sectional area $A$ of the sample.

$$
C=\frac{F_{f}}{A}
$$

Apparent modulus $E_{c}$ is defined as the change in stress divided by the change in strain for the linear portion of the stress-strain curve.

$$
\begin{gathered}
E_{c}=\frac{\Delta \text { stress }}{\Delta \text { strain }}=\frac{d S}{d x} \\
\text { stress }=\frac{F}{A}=S
\end{gathered}
$$




$$
\text { strain }=\frac{\Delta D}{H}(100 \%)=x
$$

where $\Delta D$ is the deformation of the specimen, $H$ is the initial height of the specimen, and $F$ is the load at any point during the test in the initial linear portion of the curve. Finally, the energy absorbed per unit volume $W$ by the specimen is defined as the area under the plateau region of the stress-strain curve $T(L)$ up to any desired strain $x$.

$$
W=\int_{0}^{x} T(L) d L
$$

Equation 2.6 can only be used if the cross-section of the sample being compressed stays constant throughout the compression test. In the event that the cross-section changes, Equation 2.6 can no longer be used because the area $A$ in Equation 2.2 is no longer constant. This results in false stress values in the stress-strain curve $T(L)$. 


\section{Chapter 3}

\section{Experimental Method}

After consulting the aformentioned ASTM methods and general theory of foam compression, it was noted that none of the methods are strictly applicable to carbon foam. Thus, the following experimental procedure has been proposed as a compression test method for carbon foams. This single test method provides results for yield strength, Young's Modulus, as well as energy absorption.

\subsection{Test Specimen}

Specimens shall be a right cylinder. Great care must be taken to insure that the faces of the right cylinder are parallel to each other and perpendicular to the cylindrical sides. The diameter of each cylinder shall be $25 \mathrm{~mm}$ (1in) and the height shall be between 25-50mm (1-2 inches).

\subsection{Sample Preparation}

The specimens should be conditioned for 24 hours in the room in which the test is performed.

\subsection{Testing Apparatus}

Compression tests on the carbon foam have been successfully performed using, for example, an Instron 5869 load frame fitted with a $50 \mathrm{kN}$ load cell. A piston assembly 
has been constructed out of stainless steel in order to control the sample cross-section during compression testing. This defines a one-dimensional volume change as the samples are compressed.

A hollow, stainless steel cylinder has been turned on a lathe with an inside diameter of $25.4254 \mathrm{~mm} \pm 0.0254 \mathrm{~mm}(1.001 \pm 0.001 \mathrm{in})$. An $88.9 \mathrm{~mm}$ (3.50in) long solid plunger and a $12.7 \mathrm{~mm}(0.50 \mathrm{in})$ long solid plug were also constructed with an outside diameter of $25.3746 \mathrm{~mm} \pm 0.0254 \mathrm{~mm}(0.999 \pm 0.001 \mathrm{in})$. The plug is employed as a false bottom for the sample chamber cavity to provide a stable base for the specimen and to ensure that the crushed specimen can be removed. A photograph of the complete assembly can be seen in Figure 3.1 and a schematic of the piston assembly crushing a carbon foam sample along with its resulting stress-strain plot can be seen in Figure 3.2. The foam is compressed between the solid plunger and the solid plug to ensure a onedimensional volume change between two parallel surfaces.

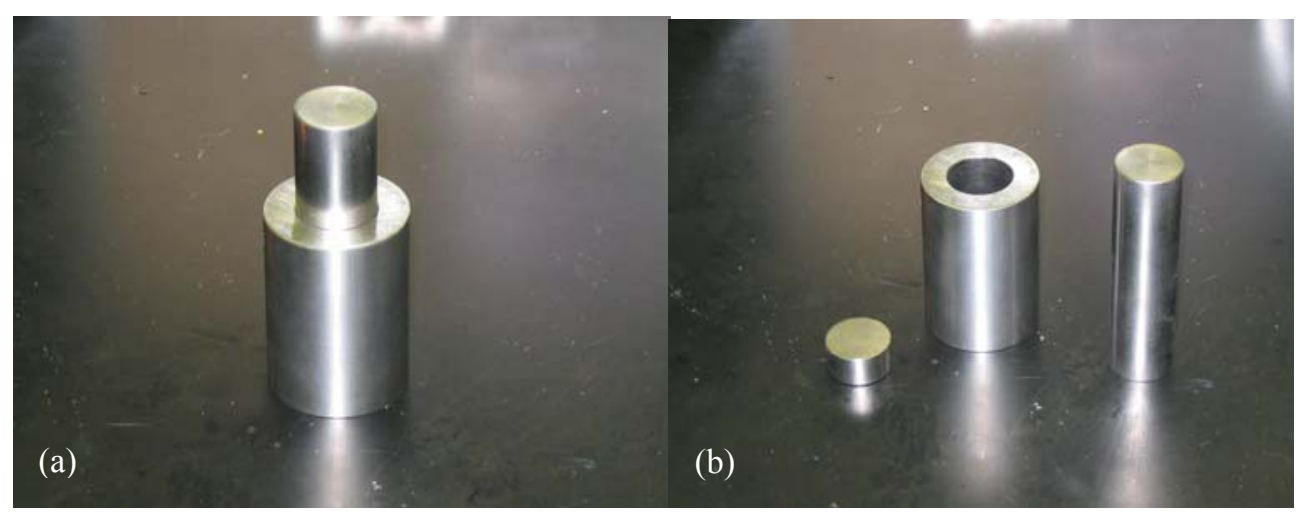

Figure 3.1. Photographs of the piston-assembly used in compression tests as a complete assembly (a), and its individual parts (b). 


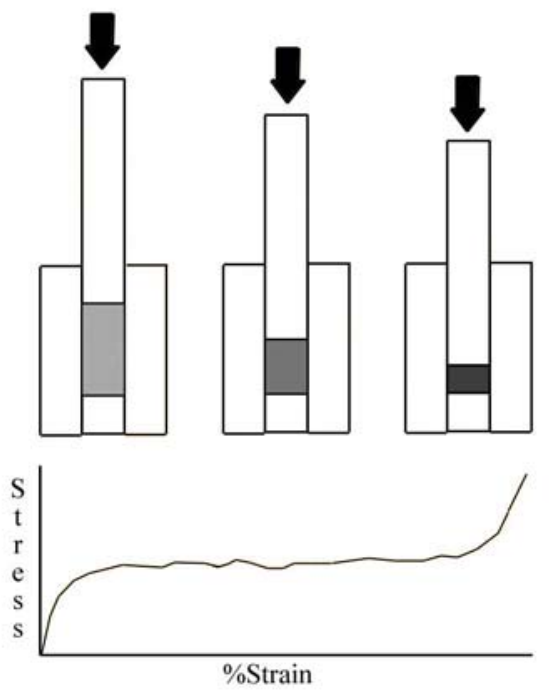

Figure 3.2. Schematic of piston assembly and corresponding Stress vs. Strain plot.

\subsection{Procedure}

The height and diameter of each specimen are recorded as an average of 3 different measurements using a caliper to the nearest \pm 0.001 in $( \pm 0.0254 \mathrm{~mm})$. The measurement shall be taken when the faces of the caliper first come into contact with the specimen as there should be no force from the caliper applied to the specimen. One diameter measurement is taken across the middle of cylinder, and the other two measurements are taken on opposite ends of the specimen. The height measurements are taken similarly, one across the center of the cylinder face, and two at opposite ends. The specimen weight $( \pm 0.01 \mathrm{~g})$ shall also be taken so that bulk density can be calculated. The specimen shall be placed in the piston assembly, assuring that the loaded ends are parallel and no binding can occur between the piston and the cylinder. The piston assembly is placed between the platens, being sure that the assembly is centered on the lower platen. 
The platens shall compress at $1 \mathrm{~mm} / \mathrm{min}$ to assure that there is no air resistance being recorded as air escapes the cylinder.

\subsection{Calculations}

The yield strength (or compressive strength) $C(\mathrm{MPa})$ is defined as the maximum load $F_{m}(\mathrm{~N})$ of the linear portion of the stress-strain curve divided by the apparent crosssectional area $A_{c}\left(\mathrm{~mm}^{2}\right)$ of the foam sample being tested.

$$
C=\frac{F_{m}}{A_{c}}
$$

The Young's Modulus $E_{c}(\mathrm{MPa})$ is the slope of the linear portion of the plot and is defined as the change in stress divided by the change in strain.

$$
E_{c}=\frac{y_{1}-y_{2}}{\left(\frac{x_{1}-x_{2}}{100}\right)}
$$

where $x_{1}, y_{1}$ is a set of data for strain (\% strain) and stress (MPa) at an instant, and $x_{2}, y_{2}$ is a set of data for strain and stress at another instant (both are on the linear portion of the curve).

The total energy absorbed per unit volume is found by integrating the area under the Stress vs. Strain plot. Integration can be carried out using the Newton-Cotes closed integration formula where: 


$$
\begin{gathered}
x_{i}=\frac{x_{n+1}-x_{n}}{100} \\
y_{i}=\frac{y_{n}+y_{n+1}}{2} \\
A=\sum_{1}^{i} x_{i} y_{i}
\end{gathered}
$$

The total area, $A$, under the stress-strain plot, which is the total energy absorbed by the foam, is calculated by the summation of the products of each individual piece of area. The individual pieces are defined as the product of $x_{i}$ and $y_{i}$. The term, $x_{i}$, is defined as the difference between two consecutive data points of strain (\%), where the $y_{i}$ term is the arithmetic mean of two consecutive data points of stress (MPa). The area under the plot is the total energy absorbed per unit volume expressed in $\mathrm{MJ} / \mathrm{m}^{3}$, and dividing the total energy by the sample density results in specific energy $(\mathrm{kJ} / \mathrm{kg})$. 


\section{Chapter 4}

\section{Preliminary Results}

Numerous tests have been successfully performed using the proposed compression test method for compressing carbon foams. However, nearly all of the tests were done in the early stage of development of this technique. Statistical analysis was never performed on any batch of similar samples to compare the consistency of the results because of the lack of availability of such samples.

Many tests have been performed on various types of carbon foams using this method. These foams have differed greatly in bulk density, cell size and shape, as well as the material from which the foam was produced. There have been many specific testing sessions in which the constrained test was used to obtain data for different carbon foams. Two of the most recent were done as part of a round robin test for the United States Air Force Research Laboratory. The first session was comprised of testing foams from Poco Graphite Incorporated; Touchstone Research Laboratories (TRL); and Materials and Electrochemical Research (MER) Corporation. The second such session included carbon foams from Ultramet and Fiber Materials Incorporated (FMI). No further information was supplied concerning the manufacturing processes used for any of these carbon foam samples. It should also be noted that complete information regarding other laboratories involved in the round robin test, their testing procedures, and their results are not available at the time of this writing.

The foam blocks, which varied in size from manufacturer to manufacturer, were taken from a large piece of foam for each individual foam sample. The large foam pieces were cut into smaller blocks and distributed by the Air Force to the different laboratories 
involved in the round robin test. Therefore all laboratories received a similar block of foam, which ultimately came from the same parent piece of carbon foam from each manufacturer. However, the blocks of foam received by WVU for testing were small and did not allow for more than two samples to be prepared and compressed due to physical size limitations.

The first session's results, shown in Table 4.1, reveal that the Poco graphitized carbon foam sample is much softer and pliable than that from MER or TRL, making it behave differently in the crushing test. The crushed Poco samples did not break apart, nor did they crack or fracture in any way. The volumes of the soft, graphitized samples were simply reduced, while their cross section remained intact. MER Corporation also included a graphitized carbon foam sample (labeled MERG) that behaved in a very similar manner.

The sample from Touchstone Research Laboratories was a much lighter, brittle foam and behaved as such. The stress-strain plot was very jagged, and not constant. This behavior suggests non-uniformity in the cell structure of the foam. The foam matrices of the samples were completely destroyed in the compression tests, leaving behind nothing more than carbon dust in the chamber.

The MER sample proved to be very difficult to cut, and as a consequence only one useful crush specimen could be obtained. However, the one sample that was compressed yielded a successful compression test. It also behaved as a brittle foam, in that the stress-strain curve was not smooth, and the foam matrix was completely destroyed in the test. The results of this compression testing session can be seen in Table 4.1. 
Table 4.1. Compression Performance of Carbon Foam Samples.

\begin{tabular}{|c|c|c|c|c|c|c|c|}
\hline Specimen & $\begin{array}{c}\text { Bulk } \\
\text { Density }\end{array}$ & $\begin{array}{l}\text { Volumetric } \\
\text { Energy } \\
\text { Absorption }\end{array}$ & $\begin{array}{c}\text { Specific } \\
\text { Energy } \\
\text { Absorption }\end{array}$ & Modulus & $\begin{array}{c}\text { Yield } \\
\text { Strength }\end{array}$ & $\begin{array}{l}\text { Specific } \\
\text { Modulus }\end{array}$ & $\begin{array}{c}\text { Specific } \\
\text { Yield } \\
\text { Strength } \\
\end{array}$ \\
\hline & $\left(\mathrm{g} / \mathrm{cm}^{3}\right)$ & $\left(\mathbf{M J} / \mathrm{m}^{3}\right)$ & $(\mathrm{kJ} / \mathrm{kg})$ & (MPa) & (MPa) & $\left(\mathrm{kPa}^{\prime} \mathrm{m}^{3} / \mathrm{kg}\right)$ & $\left(\mathrm{kPa}^{\prime} \mathrm{m}^{3} / \mathrm{kg}\right)$ \\
\hline POCO 1 & 0.62 & 1.31 & 2.1 & 160 & 2.25 & 260 & 3.6 \\
\hline $\mathrm{POCO} 2$ & 0.62 & 1.41 & 2.3 & 124 & 2.06 & 200 & 3.3 \\
\hline $\begin{array}{l}\text { POCO } \\
\text { Average } \\
\end{array}$ & 0.62 & 1.36 & 2.2 & 142 & 2.16 & 230 & 3.5 \\
\hline TRL 1 & 0.30 & 2.93 & 9.8 & 136 & 6.23 & 450 & 20.8 \\
\hline TRL 2 & 0.30 & 3.18 & 10.6 & 147 & 6.15 & 490 & 20.5 \\
\hline $\begin{array}{l}\text { TRL } \\
\text { Average } \\
\end{array}$ & 0.30 & 3.06 & 10.2 & 142 & 6.19 & 470 & 20.6 \\
\hline MER & 0.71 & 22.51 & 31.7 & 541 & 26.36 & 760 & 37.1 \\
\hline MERG 1 & 0.48 & 0.86 & 1.79 & 74 & 1.40 & 154 & 2.9 \\
\hline MERG 2 & 0.51 & 1.04 & 2.04 & 88 & 1.58 & 173 & 3.1 \\
\hline $\begin{array}{l}\text { MERG } \\
\text { Average }\end{array}$ & 0.50 & 0.95 & 1.92 & 81 & 1.49 & 164 & 3.0 \\
\hline
\end{tabular}

The Touchstone foam had the lowest apparent or bulk density, yet was surprisingly strong. The bulk density of the POCO, MER, and MERG foams were comparable, but the ungraphitized MER foam was much stronger and absorbed nearly 20 times more energy per unit volume. This is due, in part, to the fact that the graphitized POCO and MERG foams were soft and pliable.

During the second compression testing session, all three samples, from both FMI and Ultramet, behaved as brittle foams when crushed. The FMI foam was much stronger than that of Ultramet. These results are shown in Table 4.2. This was expected since the FMI had a bulk density over three times greater than the Ultramet foam.

Due to the small dimensions of the FMI foam block, limitations allowed only one sample to be cut from the original block, but the single compression test performed extremely well. Unfortunately some problems arose while testing both Ultramet samples. 
Neither of the Ultramet compression tests ran completely to the densification range of the stress-strain plot. The first test was not completed due to equipment failure caused by a power outage. The resulting plot can be seen in Figure 4.1. The second test was incomplete due to operator error in estimating the approximate strain for densification. These problems prevented the complete energy absorption calculations to be carried out for either sample. However, all three samples allowed for the determination of the modulus and yield stress as shown in Table 4.2.

Table 4.2. Compression Performance of Carbon Foam Samples.

\begin{tabular}{|c|c|c|c|c|c|c|c|}
\hline Specimen & $\begin{array}{c}\text { Bulk } \\
\text { Density }\end{array}$ & $\begin{array}{c}\text { Volumetric } \\
\text { Energy } \\
\text { Absorption }\end{array}$ & $\begin{array}{c}\text { Specific } \\
\text { Energy } \\
\text { Absorption }\end{array}$ & Modulus & $\begin{array}{c}\text { Yield } \\
\text { Stress }\end{array}$ & $\begin{array}{c}\text { Specific } \\
\text { Modulus }\end{array}$ & $\begin{array}{c}\text { Specific } \\
\text { Yield } \\
\text { Stress }\end{array}$ \\
\hline & $\mathbf{( g / \mathbf { c m } ^ { 3 } )}$ & $\left.\mathbf{( M J} / \mathbf{m}^{\mathbf{3}}\right)$ & $\mathbf{( k J / k g )}$ & $\mathbf{( M P a )}$ & $\mathbf{( M P a )}$ & $\mathbf{( k P a \cdot \mathbf { m } ^ { \mathbf { 3 } } \mathbf { / k g } )}$ & $\mathbf{( k P a \cdot \mathbf { m } ^ { \mathbf { 3 } } / \mathbf { k g } )}$ \\
\hline FMI & 0.50 & 4.04 & 8.08 & 326 & 4.32 & 652 & 8.64 \\
\hline & & & & & & & \\
\hline Ultramet 1 & 0.16 & - & - & 46.7 & 0.71 & 292 & 4.44 \\
\hline Ultramet 2 & 0.16 & - & - & 35.7 & 0.68 & 223 & 4.25 \\
\hline $\begin{array}{c}\text { Ultramet } \\
\text { Average }\end{array}$ & $\mathbf{0 . 1 6}$ & - & - & $\mathbf{4 1 . 2}$ & $\mathbf{0 . 7 0}$ & $\mathbf{2 5 8}$ & $\mathbf{4 . 3 5}$ \\
\hline
\end{tabular}

Due to the wide differences in bulk density of these two foams, it is not valid to compare them directly. However, all foam samples did behave as expected, with the FMI sample having a much higher yield stress and modulus. The total energy absorption was not calculated for either Ultramet foam sample because of the problems that occurred during the test, which did not allow either test to be completed. 


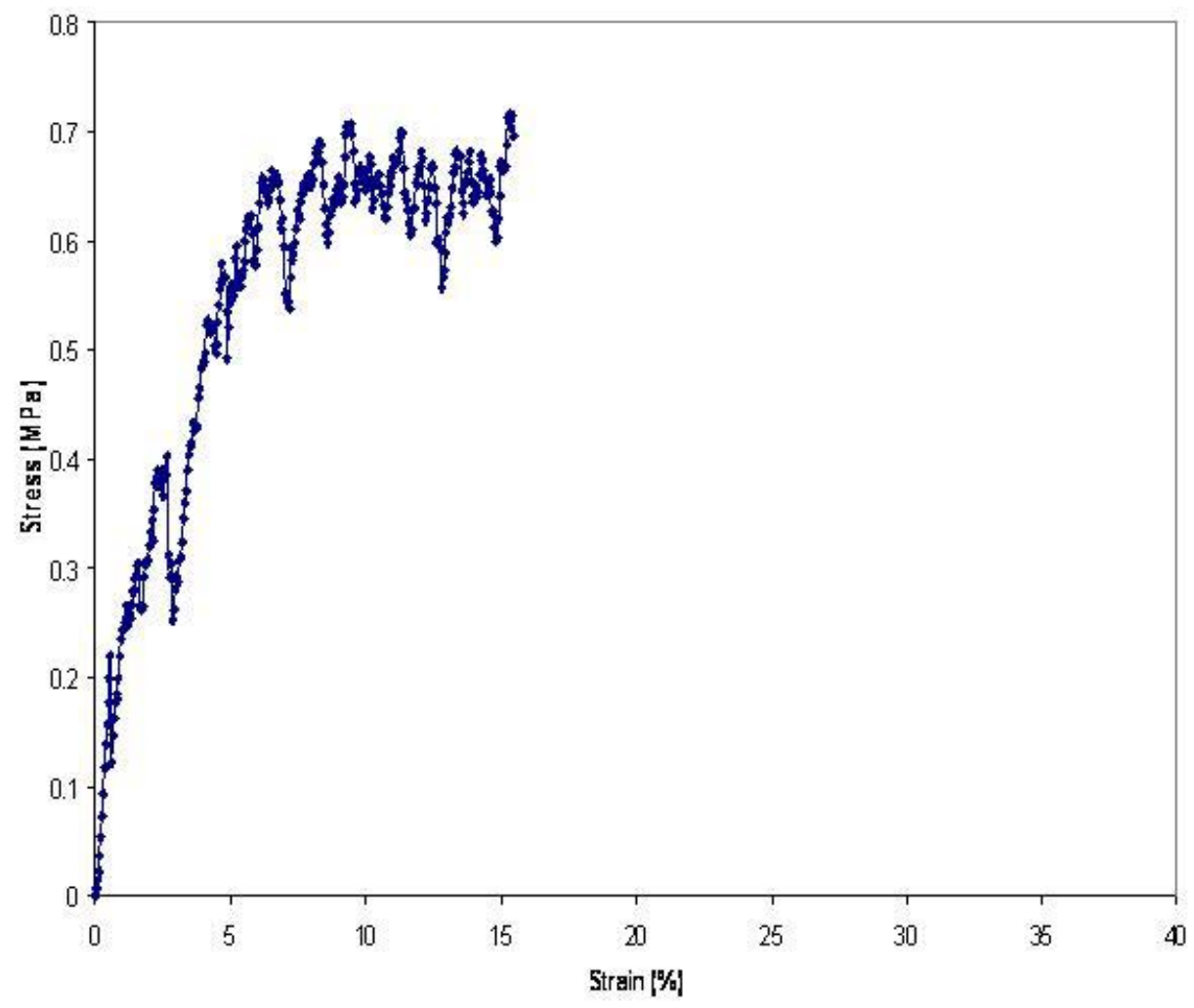

Figure 4.1. Plot Showing Incomplete Stress/Strain Data of Ultramet Foam Sample 1.

The data in Tables 4.1 and 4.2 show that, indeed, yield strength, modulus, and total absorbed energy can be determined using the one single compression testing method proposed. However, the amount of data is not sufficient to support a statistical analysis comprising of standard deviation, variance, or reproducibility due to the small number of samples that were tested. 


\section{Chapter 5}

\section{Project Objectives}

Statistical analysis must be done on this compression testing method in order to show that dependable and reproducible yield strength results can be aquired. Also, the results from the compression method described here must be compared to those from the more conventional unconstrained test. There will be a slight discrepancy in the numbers between any number of samples, even of the same foam, due to the anisotropy of both the matrix of the foam and the carbon material itself. Thus solid statistical evaluations must be completed. Finally, comparison between yield strength results from conventional unconstrained testing without the piston compression assembly and constrained testing using the piston compression assembly must be done in order to assess the utility of the constrained piston compression test.

Unconstrained compression tests will be performed to allow statistical information to be gathered about carbon foam compression testing. This step will provide information about the reproducibility, standard deviation, and averages of the properties being reported. The proper number of samples that must be tested during a single session can also be determined. In addition to statistical analysis, the constrained compression test will also be performed on the foam samples in order to determine what influences the piston assembly may have on the compression test results. It is imperative to determine if the piston compression assembly adds any significant forces that would in any way influence the results. The results of the unconstrained test will be compared to the results of the constrained test to determine if the piston assembly is influencing the results of the compression test. These tests will be performed using large pieces of foam 
that have been received from GrafTech International. The pieces are so large that dozens of test specimens can be cut from a single block. GrafTech has supplied foams with nominal densities between 2 and $35 \mathrm{lb} / \mathrm{ft}^{3}$.

In order to perform the unconstrained test, foam samples will be cut using the same sample preparation techniques as used for the constrained tests. These samples will be compressed alone, without the use of the piston compression assembly. The samples

will be centered on the lower platen of the Instron 5869 load frame and compressed. These results will not give total energy absorption for most samples because if a sample breaks, or its cross-sectional area changes, the resulting stress calculations will not correspond to the force applied to an already specified area. However, accurate yield strength and modulus results should be obtained.

These yield strength and modulus results will be compared to the same results of the constrained piston compression assembly test to determine if the constrained piston test is giving accurate results.

\subsection{Experimental Plan}

The primary objective of these experiments is to develop a standard compression test for all carbon foams and to perform statistical analysis of the resultant data. The tasks to be completed for these experiments are as follows:

- develop the compression test protocol and the experimental piston assembly

- gather carbon foams of different densities from different manufacturers

- prepare all compression samples for compression testing 
- visually compare microstructures of different carbon foams using SEM micrographs

- test carbon foam samples using both the unconstrained and constrained methods

- observe compression of foam samples through an optically transparent Lexan ${ }^{\circledR}$ cylinder

- compare data from all tests for each sample including yield stress, modulus, and energy absorption (if applicable) and perform statistical analysis of the data

Samples have been collected from several sources including GrafTech International, MER Corporation, Ultramet, and WVU. These samples vary in density and cell size. They are also manufactured from different precursor materials and are processed differently. These foams were chosen because of their density differences in order to collect data from a wide variety of carbon foams.

The foam from Ultramet will be tested specifically to gain information on energy absorption. The light-weight matrix of the Ultramet foam is such that its cross-section does not change during compression. Therefore, Ultramet foam can be compressed with or without the piston assembly. This information will provide proof that the constrained piston test can give an accurate measure of total energy absorbed in a piece of carbon foam, provided the foam does not shatter upon compression. The Ultramet foam is sized such that compression test on a specimen of different height can be done as well. The initial block height is 2 in. Therefore, two sets of samples will be cut at heights of 1 in and 2 in and will be compressed using the same testing conditions to determine if the height to diameter ratio has an obvious effect on the results of the compression test. 
The foams from GrafTech will be tested in order to provide statistical information regarding the compression method. The largest number of samples possible will be cut out of the original blocks of foam in order to determine accuracy and reproducibility. GrafTech has provided carbon foams ranging in density from 2-35 $\mathrm{lb} / \mathrm{ft}^{3}$. A light-weight

foam $\left(2 \mathrm{lb} / \mathrm{ft}^{3}\right)$, a medium-weight foam $\left(10 \mathrm{lb} / \mathrm{ft}^{3}\right)$ and a heavy-weight foam $\left(20 \mathrm{lb} / \mathrm{ft}^{3}\right)$ will be tested in order to gain statistical data from each extreme density.

The MER and WVU foams will be tested in order to substantiate the test's validity for higher density foams and to acquire the best reproducibility possible.

All of these foam specimens will be prepared for SEM analysis. This microscopic analysis will be used to inspect visually the matrices of each of the foams and compare them. These micrographs will allow general statements to be made regarding any connection between bulk matrix and apparent strength.

\subsection{Safety}

Safety glasses with side shields must always be worn throughout the entire compression testing process. During sample preparation, safety glasses with side shields should be worn since pieces that break from carbon foam blocks using a hole-saw can become projectiles. Black, heavy, respiratable dust can be formed during hole-saw cutting as well. Breathing protection from fine-particles must be worn for this reason. All other safety guidelines for machine tools (drill press, band-saw...etc.) were followed according to each piece of equipment's guidelines (no loose clothing near moving parts, make sure all guides and shields are in place...etc.) 
No items (except those being compressed) will be near platens during compression testing. Safety glasses with side shields must be worn, especially during the unconstrained testing, because foam pieces can become projectiles. In case of emergency, a large, red, emergency stop button is located on the front of the Instron load frame. This emergency stop will cut power to the entire load frame. 


\section{Chapter 6}

\section{Results and Discussion}

Compression tests were performed on carbon foams from different manufacturers. Carbon foams from GrafTech International, Ltd., Materials and Electrochemical Research (MER) Corporation, Ultramet, and West Virginia University were tested in order to gather information and data from carbon foams of different precursor material, different manufacturing processes, and with different physical properties. Appendix A shows all experimental plots of stress/strain that were acquired during this study. A wide range of carbon foams were chosen in order to show that the proposed compression test can, in fact, be used on any type of carbon foam, regardless of bulk density or precursor material.

Statistical analyses were also successfully performed on three GrafTech foams with different bulk densities $\left(2,10\right.$, and $\left.20 \mathrm{lb} / \mathrm{ft}^{3}\right)$. These analyses were performed on the yield stress, modulus, and energy absorption results for each of the three foams. The group of GrafTech foams allowed enough material for 20 to 50 specimens to be tested at each density level. This insured that confidence intervals could be determined and reported. The other foams did not provide enough material for confidence intervals, however, sample means, and sample standard deviations were calculated. The results showed that the yield stress and modulus numbers for the foams followed a normal distribution and that the energy absorption followed a chi-square distribution. These results help to confirm that, indeed, the energy absorbed by carbon foams resembles more of a lower limit value instead of an average value supported by a normal distribution. 
The foams from Ultramet, MER Corporation, and WVU were tested simply to verify that the compression test could, in fact, be completed on foams with different densities that were manufactured from different precursor material. There was not enough material to complete an entire statistical analysis on the data, but a mean and standard deviation was calculated for each set of data.

\subsection{GrafTech Foam Results}

GrafTech provided several foams of different density to be used for a statistical analysis. The three foams chosen had densities of 2,10 , and $20 \mathrm{lb} / \mathrm{ft}^{3}$. Each of these foams was tested using both unconstrained and constrained methods in order to compare the results from each technique. Specifically, the yield stress and modulus were compared to determine if the piston assembly influenced the results of the compression test. The results of these tests are presented below.

\subsubsection{GrafTech FPA-02 Results}

The $2-\mathrm{lb} / \mathrm{ft}^{3}\left(0.032 \mathrm{~g} / \mathrm{cm}^{3}\right)$ bulk density GrafTech foam, also known as FPA-02, (Figure 6.1) was used the most frequently because the foam is extremely lightweight and very easy to prepare. Therefore, five different sets of compression tests (each set contains 10 samples) were performed using this foam. Variables that were compared included sample height, testing speed (speed of platens), and the difference between constrained and unconstrained tests. The five sets of compression tests are indicated in 
Table 6.1, and the results of the compression tests are shown in Table 6.2. As seen in Figure 6.1, the FPA-02 foam is an open-cell structure with relatively large cells of nearly uniform dimension. The foam shows slight cell elongation in the foaming direction.
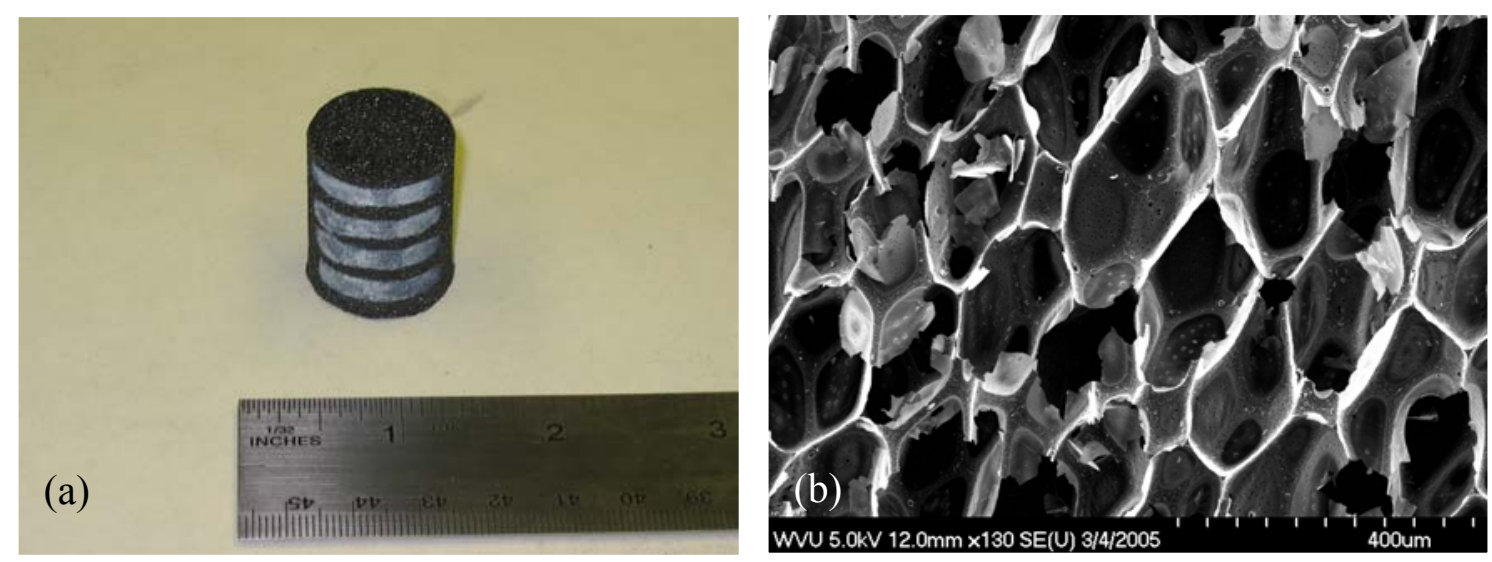

Figure 6.1. Image of FPA-02 with white lines drawn on for reference during testing (a), and (b) SEM micrograph of FPA-02.

Table 6.1. Parameters for Compression Tests of FPA-02 Carbon Foam

\begin{tabular}{|l|ccc|}
\hline & $\begin{array}{c}\text { Test } \\
\text { Type }\end{array}$ & $\begin{array}{c}\text { Sample } \\
\text { Height }(\mathbf{c m})\end{array}$ & $\begin{array}{c}\text { Platen Speed } \\
(\mathbf{m m} / \mathbf{m i n})\end{array}$ \\
\hline Test 2.1 & Unconstrained & 2.54 & 1 \\
Test 2.2 & Unconstrained & 2.54 & 2 \\
Test 2.3 & Constrained & 2.54 & 1 \\
Test 2.4 & Constrained & 2.54 & 2 \\
Test 2.5 & Constrained & 5.08 & 2 \\
\hline
\end{tabular}

A rough population mean, along with unbiased standard deviation and variance were calculated using all available data from FPA-02 since all values were extremely close together. These values are labeled in Table 6.2 under "combined." These 50 values were combined and provided a histogram (represented in Figure 6.2a) which followed a normal distribution. Using a normal distribution, various confidence intervals were 
calculated and are shown in Table 6.3. The same procedure was performed on modulus values as well.
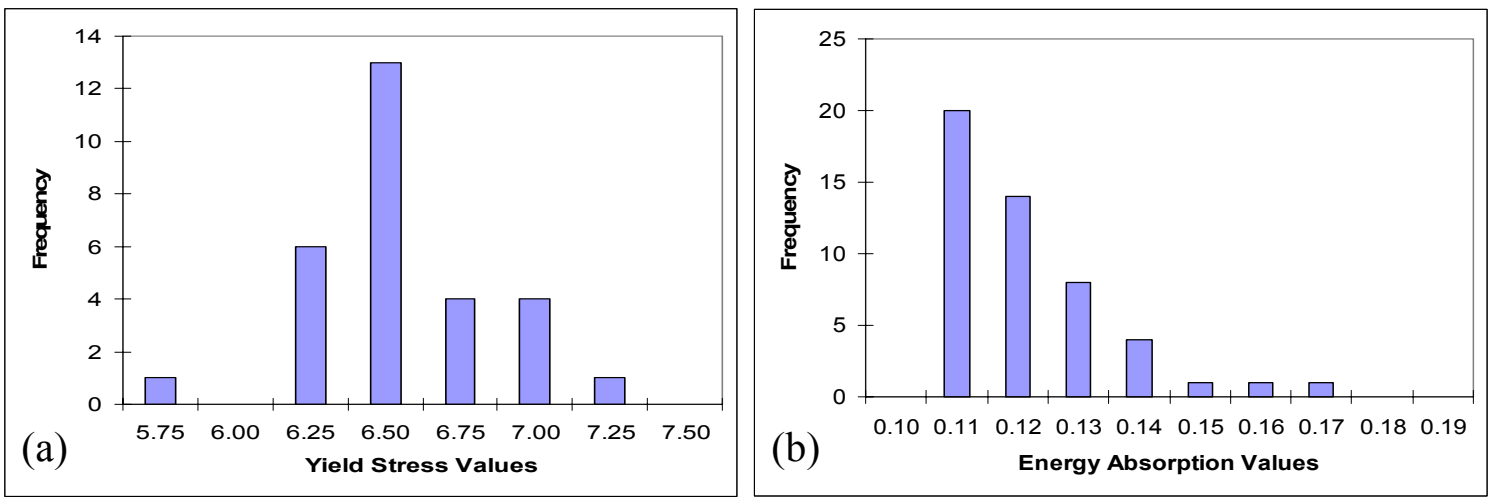

Figure 6.2. Histograms Showing Shape of Distributions for Yield Stress (a) and Energy Absorption Values(b) for all Foams Tested.

The histogram for energy absorption (represented in Figure 6.2b), however, did not follow a normal distribution, but resembled a chi-square distribution. The chi-square distribution gives confidence intervals about the variance of the set of samples, not the mean value. Therefore, the confidence interval was calculated and a range was found for the unbiased variance of the 50 samples. The confidence interval was found to be $50 \%$ and the variance, $\sigma^{2}$, was found to be between $0.00010 \leq \sigma^{2} \leq 0.00013\left(\mathrm{MJ} / \mathrm{m}^{3}\right)^{2}$. The standard deviation $(\sigma)$ is the square root of the variance. Both of these values give an indication of how much spread is in the data, in other words, how much the data vary from the mean value. The smaller these values, the closer the individual measurements are to each other and to the corresponding mean.

Compression tests were also documented using photos taken at normal intervals during unconstrained compression tests. These photos were taken to observe differences in failure between foams of different density. The photos from FPA-02 are shown in Figure 6.3. 
Table 6.2. Mean Results from Each Set of Compression Tests for FPA-02 Foam

\begin{tabular}{|c|cccc|}
\hline & $\begin{array}{c}\text { Measured Bulk } \\
\text { Density }\left(\mathbf{g} / \mathbf{c m}^{3}\right)\end{array}$ & $\begin{array}{c}\text { Yield } \\
\text { Stress }(\mathbf{M P a})\end{array}$ & $\begin{array}{c}\text { Modulus } \\
(\mathbf{M P a})\end{array}$ & $\begin{array}{c}\text { Energy } \\
\text { Absorption }\left(\mathbf{M J} / \mathbf{m}^{\mathbf{3}}\right)\end{array}$ \\
\hline Test 2.1 & $\mathbf{0 . 0 2 4}$ & $\mathbf{0 . 1 4 2}$ & $\mathbf{2 . 8 3}$ & $\mathbf{0 . 1 1 3}$ \\
Std Dev. & & 0.005 & 0.65 & 0.004 \\
Variance & & 0.000 & 0.42 & 0.000 \\
\hline Test 2.2 & $\mathbf{0 . 0 2 4}$ & $\mathbf{0 . 1 4 1}$ & $\mathbf{3 . 1 5}$ & $\mathbf{0 . 1 1 5}$ \\
Std Dev. & & 0.005 & 0.56 & 0.004 \\
Variance & & 0.000 & 0.32 & 0.000 \\
\hline Test 2.3 & $\mathbf{0 . 0 2 4}$ & $\mathbf{0 . 1 3 5}$ & $\mathbf{2 . 5 2}$ & $\mathbf{0 . 1 1 9}$ \\
Std Dev. & & 0.009 & 0.62 & 0.008 \\
Variance & & 0.000 & 0.38 & 0.000 \\
\hline Test 2.4 & $\mathbf{0 . 0 2 4}$ & $\mathbf{0 . 1 4 0}$ & $\mathbf{2 . 5 6}$ & $\mathbf{0 . 1 2 2}$ \\
Std Dev. & & 0.006 & 0.49 & 0.007 \\
Variance & & 0.000 & 0.24 & 0.000 \\
\hline Test 2.5 & $\mathbf{0 . 0 2 4}$ & $\mathbf{0 . 1 5 3}$ & $\mathbf{3 . 4 9}$ & $\mathbf{0 . 1 3 4}$ \\
Std Dev. & & 0.035 & 1.18 & 0.014 \\
Variance & & 0.001 & 1.38 & 0.000 \\
\hline Combined & & $\mathbf{0 . 1 4 2}$ & $\mathbf{2 . 9 1}$ & $\mathbf{0 . 1 2 1}$ \\
Std Dev. & & 0.017 & 0.79 & 0.011 \\
Variance & & 0.000 & 0.63 & 0.000 \\
\hline
\end{tabular}

Table 6.3. Overall Yield Stress, Modulus, and Energy Absorption Confidence Intervals for FPA-02

\begin{tabular}{|c|c|c|c|c|}
\hline & $\begin{array}{c}\text { Confidence } \\
\text { Interval }\end{array}$ & Mean & Error & $\%$ Error \\
\hline \multirow[t]{4}{*}{ Yield Stress (MPa) } & $95 \%$ & 0.142 & $\pm \mathbf{0 . 0 0 5}$ & 3.30 \\
\hline & $90 \%$ & 0.142 & \pm 0.004 & 2.76 \\
\hline & $80 \%$ & 0.142 & $\pm \mathbf{0 . 0 0 3}$ & 2.15 \\
\hline & $75 \%$ & 0.142 & $\pm \mathbf{0 . 0 0 2}$ & 1.94 \\
\hline \multirow[t]{4}{*}{ Modulus (MPa) } & $95 \%$ & 2.91 & \pm 0.22 & 7.55 \\
\hline & $90 \%$ & 2.91 & \pm 0.18 & 6.32 \\
\hline & $80 \%$ & 2.91 & \pm 0.14 & 4.93 \\
\hline & $75 \%$ & 2.91 & $\pm \mathbf{0 . 1 3}$ & 4.43 \\
\hline $\begin{array}{c}\text { Energy Absorption } \\
\left(\mathbf{M J} / \mathbf{m}^{3}\right)\end{array}$ & $50 \%$ & \multicolumn{3}{|c|}{$\begin{array}{c}\text { Variance }\left(\mathrm{MJ} / \mathrm{m}^{3}\right)^{2} \\
0.00010 \leq \sigma^{2} \leq 0.00013\end{array}$} \\
\hline
\end{tabular}


These photos suggest that FPA-02 fails one layer at a time from the edges towards the center of the sample and that the cross-sectional area remains constant. Knowing this fact, energy absorption numbers can be obtained and compared from both the constrained and unconstrained tests. Therefore the results in Table 6.2 can be used to compare yield stress, modulus, and energy absorption for both unconstrained and constrained piston tests since the cross-sectional area of the sample does not change during compression for either method.
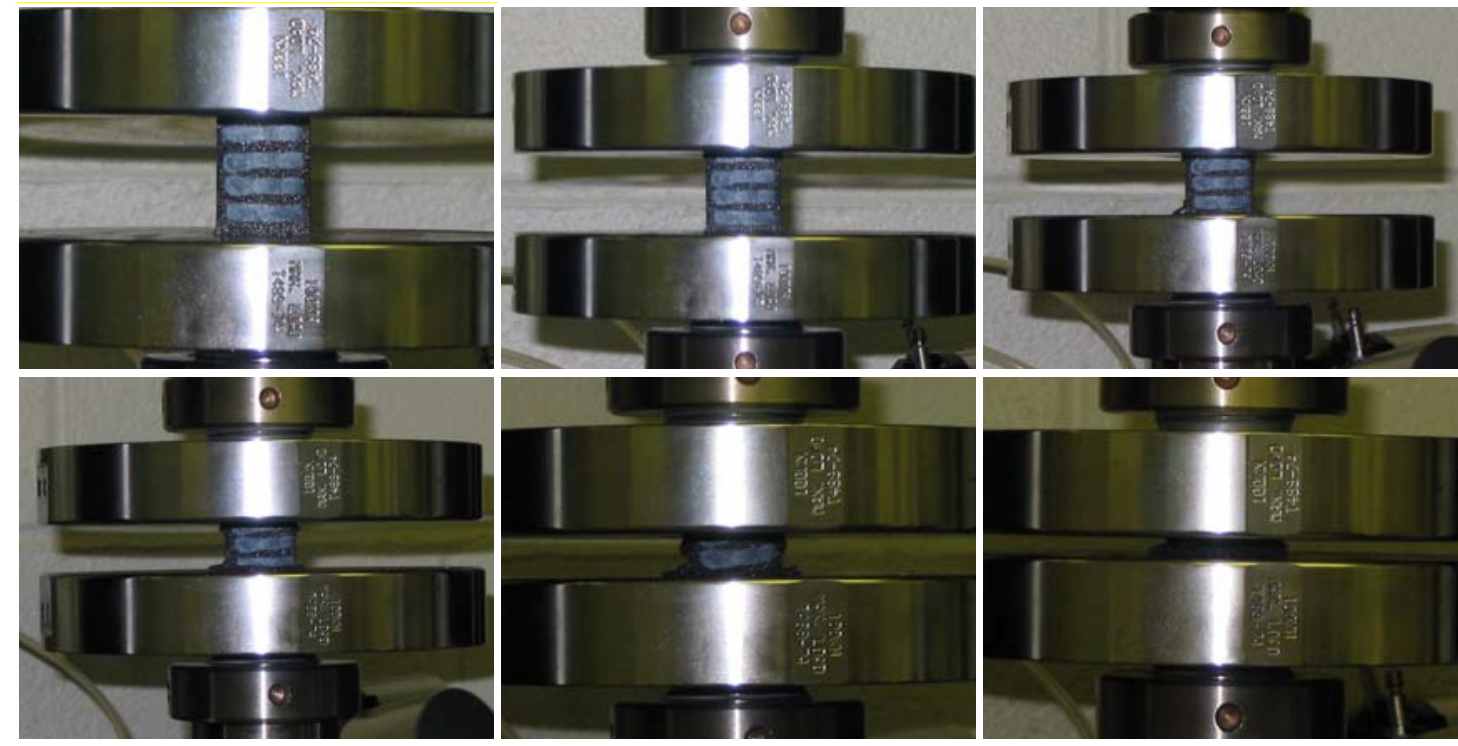

Figure 6.3. Photos taken at regular intervals (beginning at upper left) during an unconstrained compression test of FPA-02 carbon foam. (FPA-02 carbon foam courtesy of GrafTech International, Ltd.)

Comparing the micrograph in Figure $6.1 \mathrm{~b}$ with the compression results, it can be seen that this is the weakest foam of the group. The cell walls are extremely thin and thus, the yield stress is the lowest. Further investigation of the photo shows that the cells are open and interconnected, and the cell walls appear to contain smaller holes. These observations explain why FPA-02 is the weakest of the GrafTech foams tested. 
The data presented in Table 6.2 show some interesting results as well. There is very little to no difference in any of the results of Tests 2.1 and 2.3 (the constrained and unconstrained tests). This suggests that the piston assembly has no effect on the test. Moreover, all of the values for the runs that contain a 1-inch tall sample have smaller standard deviations than Test 2.5 whose sample height is 2 inches. Thus 1-inch sample heights produce a better cluster of data than 2 inch sample heights. It is also important to note that the yield stress and modulus values are not affected by the speed of the platen movement, at least over the speed range tested here.

\subsubsection{GrafTech FPA-10 Results}

GrafTech's $10-1 \mathrm{~b} / \mathrm{ft}^{3}\left(0.16 \mathrm{~g} / \mathrm{cm}^{3}\right)$ foam, known as FPA-10 was also evaluated for statistical analysis. However, there was not quite as much material initially as FPA-02. Therefore, only 30 samples were tested instead of 50 . The same statistical techniques as described above for FPA-02 were used to analyze the data from FPA-10. It was found that the yield stress and modulus again followed a normal distribution. However, a student-t distribution was used since the number of samples was close to thirty. In general, when the number of samples is less than or equal to 30 , a student-t distribution is used instead of the normal distribution in order to predict more accurately the amount of

error associated with having a relatively small amount of data. FPA-10 can be seen in Figure 6.4. 
The structure of FPA-10 varies slightly from FPA-02 in that the cell walls are thicker and the cells appear rounder. The cells are still open and interconnected, and the cell walls contain numerous smaller holes.
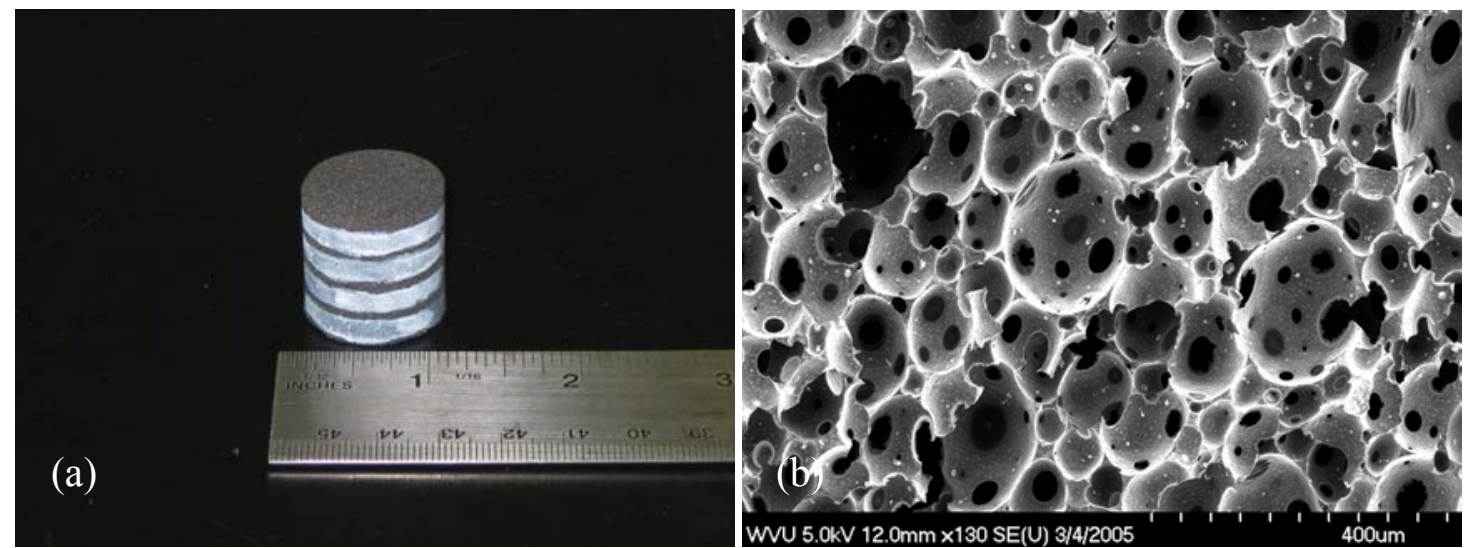

Figure 6.4. Image of FPA-10 with white lines drawn on for reference during testing (a), and (b) SEM micrograph of FPA-10.

The tests performed on FPA-10 are indicated in Table 6.4. All tests were performed using the same platen speed of $2 \mathrm{~mm} / \mathrm{min}$ and all samples were kept at a height of 1 inch. One set of samples was compressed without the piston assembly and the results were compared to two identical sets of tests using the piston assembly. Therefore, one constrained test was compared to two separate constrained tests to observe differences between the constrained and unconstrained techniques, and to observe any differences in the same test performed on two different occasions. The results from each test are shown in Table 6.5.

The series of photos taken during an unconstrained compression test of FPA-10, illustrated in Figure 6.5, shows that the higher-density foam fails in a different manner 
than FPA-02. The FPA-10 foam fails by the breaking off of large pieces and not from the gradual degradation of the outer surfaces that are in contact with the compression device.

Table 6.4. Parameters for Compression Tests of FPA-10 Carbon Foam

\begin{tabular}{|l|ccc|}
\hline & $\begin{array}{c}\text { Test } \\
\text { Type }\end{array}$ & $\begin{array}{c}\text { Sample } \\
\text { Height }(\mathbf{c m})\end{array}$ & $\begin{array}{c}\text { Platen Speed } \\
\text { (mm/min) }\end{array}$ \\
\hline Test 10.1 & Unconstrained & 2.54 & 2 \\
Test 10.2 & Constrained & 2.54 & 2 \\
Test 10.3 & Constrained & 2.54 & 2 \\
\hline
\end{tabular}

Table 6.5. Mean Results from Each Set of Compression Tests for FPA-10 Foam

\begin{tabular}{|rr|cccc|}
\hline & & $\begin{array}{c}\text { Measured Bulk } \\
\text { Density }\left(\mathbf{g} / \mathbf{c m}^{3}\right)\end{array}$ & $\begin{array}{c}\text { Yield } \\
\text { Stress }(\mathbf{M P a})\end{array}$ & $\begin{array}{c}\text { Modulus } \\
(\mathbf{M P a})\end{array}$ & $\begin{array}{c}\text { Energy } \\
\text { Absorption }\left(\mathbf{M J} / \mathbf{m}^{\mathbf{3}}\right)\end{array}$ \\
\hline Test 10.1 & & $\mathbf{0 . 1 6 9}$ & $\mathbf{6 . 4 6}$ & $\mathbf{1 8 2}$ & na \\
& Std Dev. & & 0.23 & 15 & na \\
& Variance & & 0.05 & 237 & na \\
\hline Test 10.2 & & $\mathbf{0 . 1 7 0}$ & $\mathbf{6 . 5 9}$ & $\mathbf{1 7 9}$ & $\mathbf{4 . 0 2}$ \\
& Std Dev. & & 0.35 & 28 & 0.29 \\
& Variance & & 0.12 & 784 & 0.08 \\
\hline Test 10.3 & & $\mathbf{0 . 1 6 6}$ & $\mathbf{6 . 2 4}$ & $\mathbf{1 5 5}$ & $\mathbf{3 . 8 2}$ \\
& Std Dev. & & 0.25 & 23 & 2.68 \\
& Variance & & 0.06 & 521 & 0.07 \\
\hline Combined & & $\mathbf{6 . 4 2}$ & $\mathbf{1 7 1}$ & $\mathbf{3 . 9 1}$ \\
& Std Dev. & & 0.30 & 23 & 0.29 \\
& Variance & & 0.09 & 620 & 0.08 \\
\hline
\end{tabular}

The photos show that the cross-sectional area of the foam sample is constantly changing. As the specimen is compressed, large pieces break off from the original sample. Thus the changing cross-sectional area does not allow for valid energy absorption results to be gathered from the unconstrained test. This is in contrast to the case of FPA-02, which failed by a different mechanism. Therefore, no energy absorption results are reported for the unconstrained test of FPA-10 in Table 6.5. A student-t 
distribution was used to gather confidence intervals for the yield stress and modulus, while the energy absorption followed the chi-square distribution. The confidence intervals are shown in Table 6.6.
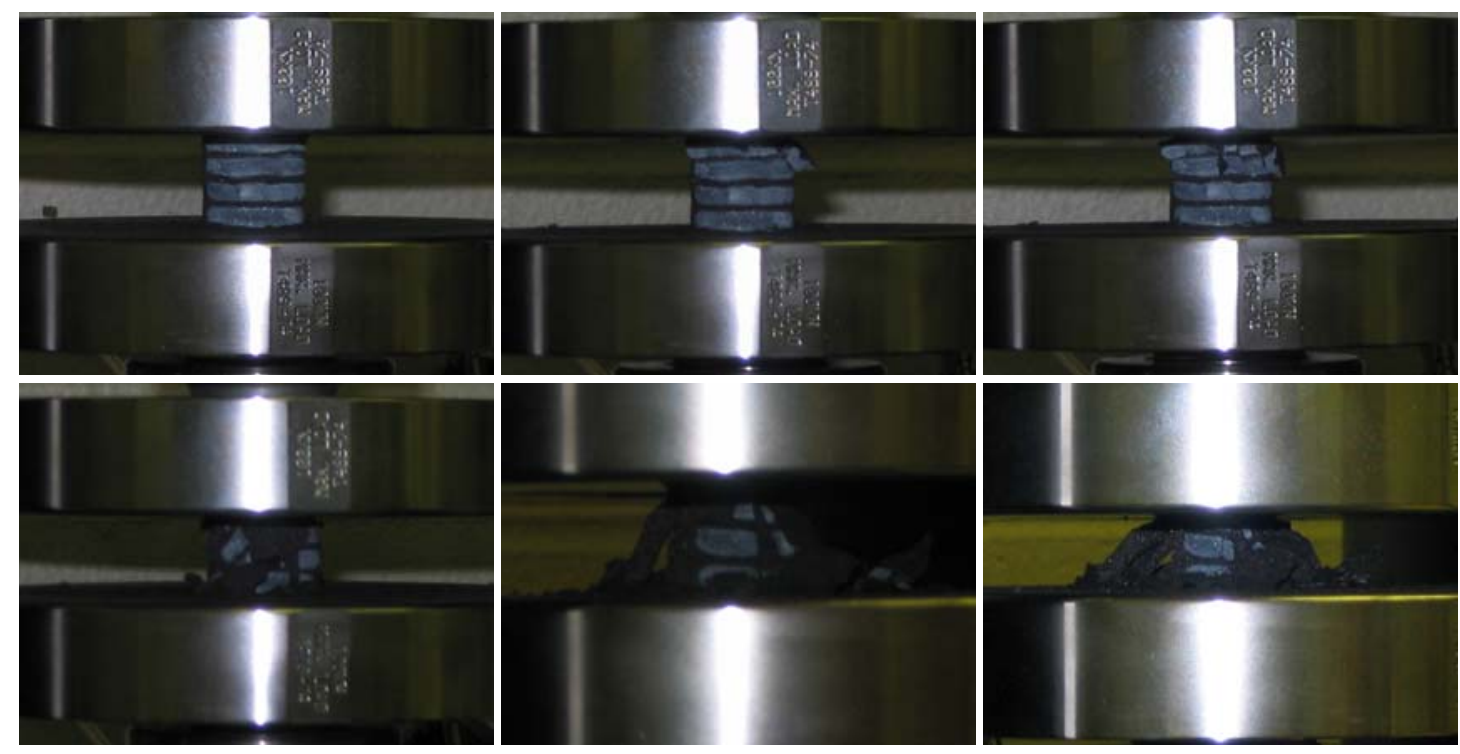

Figure 6.5. Photos taken at regular intervals (beginning at upper left) during an unconstrained compression test of FPA-10 carbon foam. (FPA-10 carbon foam courtesy of GrafTech International, Ltd.)

All three sets of data for yield stress and modulus are close to each other with the standard deviation of the yield stress being very low for all three. The two sets of data for energy absorption in the constrained tests are also very similar with low standard deviation. Low standard deviations are desired because it means the data are more precise. Good precision is important because it defines how well data can be reproduced. 
Table 6.6. Overall Yield Stress, Modulus, and Energy Absorption Confidence Intervals for FPA-10

\begin{tabular}{|c|c|c|c|c|}
\hline & $\begin{array}{c}\text { Confidence } \\
\text { Interval }\end{array}$ & Mean & Error & \% Error \\
\hline \multirow[t]{4}{*}{ Yield Stress (MPa) } & $95 \%$ & 6.42 & \pm 0.10 & 1.52 \\
\hline & $90 \%$ & 6.42 & $\pm \mathbf{0 . 0 8}$ & 1.17 \\
\hline & $80 \%$ & 6.42 & \pm 0.05 & 0.76 \\
\hline & $75 \%$ & 6.42 & \pm 0.04 & 0.61 \\
\hline \multirow[t]{4}{*}{ Modulus (MPa) } & $95 \%$ & 171 & \pm 7.99 & 4.66 \\
\hline & $90 \%$ & 171 & \pm 6.16 & 3.59 \\
\hline & $80 \%$ & 171 & \pm 4.02 & 2.35 \\
\hline & $75 \%$ & 171 & \pm 3.21 & 1.87 \\
\hline $\begin{array}{c}\text { Energy Absorption } \\
\left(\mathrm{MJ} / \mathrm{m}^{3}\right)\end{array}$ & $50 \%$ & \multicolumn{3}{|c|}{$\begin{array}{c}\text { Variance }\left(\mathrm{MJ} / \mathrm{m}^{3}\right)^{2} \\
0.06 \leq \sigma^{2} \leq \mathbf{0 . 1 0}\end{array}$} \\
\hline
\end{tabular}

\subsubsection{GrafTech FPA-20 Results}

Compression data for GrafTech's carbon foam, FPA-20, with bulk density of 20-

$\mathrm{lb} / \mathrm{ft}^{3}\left(0.32 \mathrm{~g} / \mathrm{cm}^{3}\right)$ were also evaluated for statistical analysis. The sample along with a photomicrograph of FPA-20 are shown in Figure 6.6. It can be seen that for the higherdensity foam, the cells are much smaller and the walls are thicker with far less holes. The three sets of compression tests for FPA-20 are outlined in Table 6.7. Two initial tests were performed on FPA-20 in order to compare the results from the unconstrained and constrained techniques. The third test was performed with the intent of gathering better results with closer statistics, especially to reduce the standard deviation of the modulus data and to compare a different sample height. The mean results from the tests are in Table 6.8. The confidence intervals were performed in a manner similar to those for FPA-10, in which the student-t distribution was used for yield stress and modulus, and 
the chi-square distribution was used for the energy absorption. These results are shown in Table 6.9. A comparison was performed after the Test 20.3 and better statistics were gathered due to the increase in the number of samples. The confidence intervals for the yield stress and modulus from Test 20.3 alone are shown in Table 6.10.

The structure of FPA-20 is similar to FPA-10. The main differences are that the cells are much smaller, the holes within those cell walls are much smaller, and the number of holes within the cell walls is much smaller. These observations suggest that there is more solid material in the foam matrix, which is indicated by the larger density of the foam. It should also be noted that the initial block of FPA-20 from which the samples were taken, was only 1.5 inches $(3.81 \mathrm{~cm})$ tall. The other GrafTech foams were 2 or more inches in height, allowing 2-inch tall samples to be used in the compression tests.
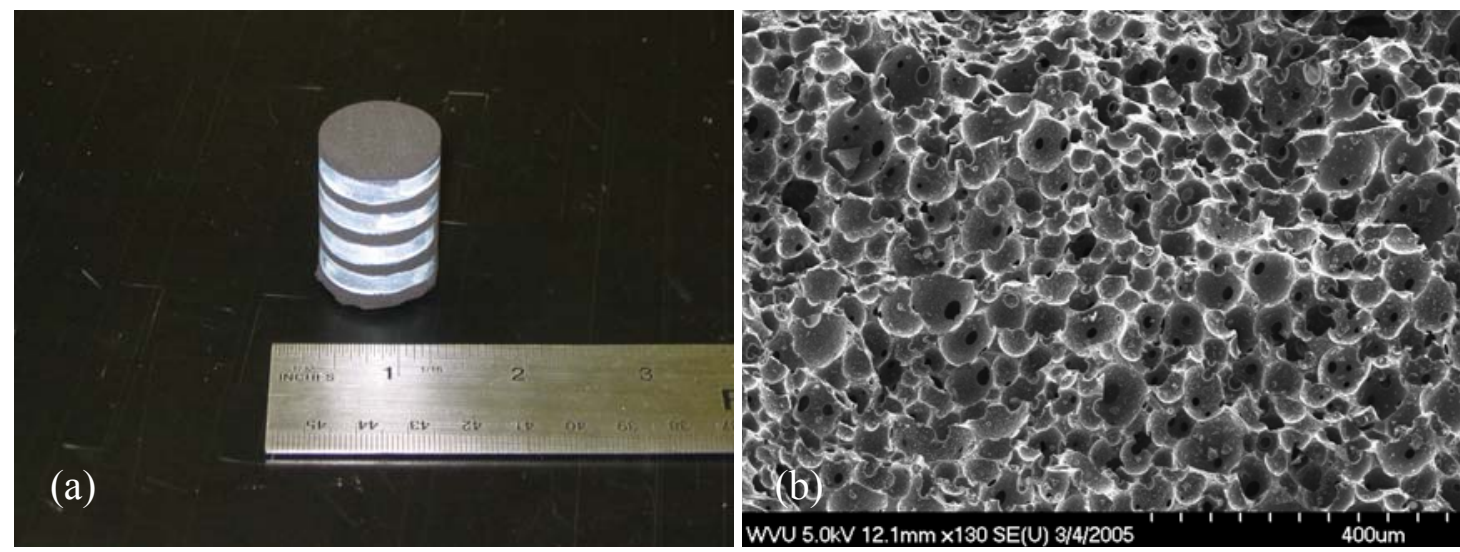

Figure 6.6. Image of FPA-20 with white lines drawn on for reference during testing (a), and (b) SEM micrograph of FPA-20.

Photos seen in Figure 6.7 were also taken during an unconstrained compression test so that failure could be visually observed. The failure of FPA-20 was much more instantaneous than that of either FPA-02 or FPA-10. The foam sample fails by allowing 
large breaks and cracks to form throughout the foam matrix. Pieces large and small fall off of the original foam sample, drastically changing its cross-sectional area. Thus the energy absorption could not be calculated from the unconstrained compression test. However, yield stress and modulus, again can be compared between all three sets of compression tests. Photos, shown in Figure 6.8, were taken of a constrained test as well. The foam was placed inside a transparent Lexan ${ }^{\circledR}$ cylinder instead of the stainless steel cylinder and compressed in a manner similar to the other constrained compressive tests. This was done to observe visually the sample within the constrained piston assembly. The observation shows that the piston is always in contact with the sample faces, and that the piston assembly does not alter or interfere with the failure mechanism of the foam. The foam is allowed to fail normally, the cylinder simply maintains the cross-section of the sample to keep it constant.

Table 6.7. Parameters for Compression Tests of FPA-20 Carbon Foam

\begin{tabular}{|l|ccc|}
\hline & $\begin{array}{c}\text { Test } \\
\text { Type }\end{array}$ & $\begin{array}{c}\text { Sample } \\
\text { Height }(\mathbf{c m})\end{array}$ & $\begin{array}{c}\text { Platen Speed } \\
(\mathbf{m m} / \mathbf{m i n})\end{array}$ \\
\hline Test 20.1 & Unconstrained & 3.81 & 2 \\
Test 20.2 & Constrained & 3.81 & 2 \\
Test 20.3 & Constrained & 2.54 & 2 \\
\hline
\end{tabular}


Table 6.8. Mean Results from Each Set of Compression Tests for FPA-20 Foam

\begin{tabular}{|rr|cccc|}
\hline & & $\begin{array}{c}\text { Measured Bulk } \\
\text { Density }\left(\mathbf{g} / \mathbf{c m}^{\mathbf{3}}\right)\end{array}$ & $\begin{array}{c}\text { Yield } \\
\text { Stress }(\mathbf{M P a})\end{array}$ & $\begin{array}{c}\text { Modulus } \\
(\mathbf{M P a})\end{array}$ & $\begin{array}{c}\text { Energy } \\
\text { Absorption }\left(\mathbf{M J} / \mathbf{m}^{\mathbf{3}}\right)\end{array}$ \\
\hline Test 20.1 & & $\mathbf{0 . 3 1 8}$ & $\mathbf{1 6 . 4}$ & $\mathbf{1 1 0 0}$ & $\mathbf{n a}$ \\
& Std Dev. & & 2.8 & 141 & na \\
& Variance & & 7.9 & 19881 & na \\
\hline Test 20.2 & & $\mathbf{0 . 3 1 7}$ & $\mathbf{1 6 . 6}$ & $\mathbf{9 1 5}$ & $\mathbf{1 1 . 0 3}$ \\
& Std Dev. & & 3.9 & 137 & 0.96 \\
& Variance & & 15.2 & 18769 & 0.92 \\
\hline Test 20.3 & & $\mathbf{0 . 3 1 5}$ & $\mathbf{1 6 . 5}$ & $\mathbf{6 9 8}$ & $\mathbf{9 . 1 5}$ \\
& Std Dev. & & 1.3 & 45 & 0.78 \\
& Variance & & 1.7 & 2025 & 0.62 \\
\hline Combined & & $\mathbf{1 6 . 5}$ & $\mathbf{9 0 3}$ & $\mathbf{1 0 . 0 5}$ \\
& & 2.7 & 203 & 1.29 \\
Std Dev. & & 7.4 & 41209 & 1.65 \\
\hline Variance & & & & \\
\hline
\end{tabular}

Table 6.9. Overall Yield Stress, Modulus, and Energy Absorption Confidence Intervals for FPA-20

\begin{tabular}{|c|c|c|c|c|}
\hline & $\begin{array}{l}\text { Confidence } \\
\text { Interval }\end{array}$ & Mean & Error & $\%$ Error \\
\hline \multirow[t]{4}{*}{ Yield Stress (MPa) } & $95 \%$ & 16.5 & \pm 0.87 & 5.27 \\
\hline & $90 \%$ & 16.5 & $\pm \mathbf{0 . 6 7}$ & 4.06 \\
\hline & $80 \%$ & 16.5 & \pm 0.44 & 2.66 \\
\hline & $75 \%$ & 16.5 & \pm 0.35 & 2.12 \\
\hline \multirow[t]{4}{*}{ Modulus (MPa) } & $95 \%$ & 903 & \pm 65.1 & 7.21 \\
\hline & $90 \%$ & 903 & $\pm \mathbf{5 0 . 2}$ & 5.56 \\
\hline & $80 \%$ & 903 & $\pm \mathbf{3 2 . 7}$ & 3.63 \\
\hline & $75 \%$ & 903 & \pm 26.2 & 2.90 \\
\hline $\begin{array}{c}\text { Energy Absorption } \\
\left(\mathrm{MJ} / \mathrm{m}^{3}\right)\end{array}$ & $50 \%$ & \multicolumn{3}{|c|}{$\begin{array}{c}\text { Variance }\left(\mathrm{MJ} / \mathrm{m}^{3}\right)^{2} \\
1.30 \leq \sigma^{2} \leq \mathbf{2 . 0 3}\end{array}$} \\
\hline
\end{tabular}



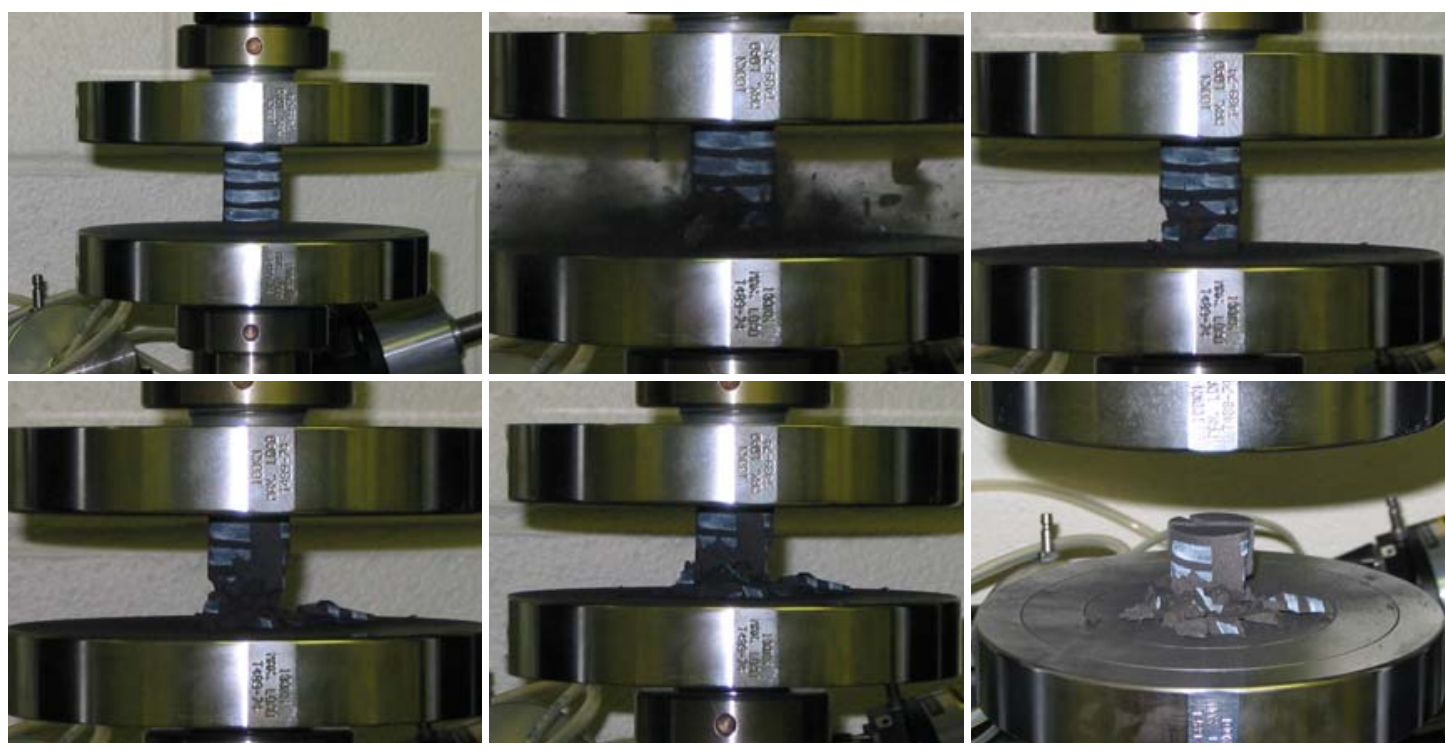

Figure 6.7. Photos taken at regular intervals (beginning at upper left) during an unconstrained compression test of FPA-20 carbon foam.

(FPA-20 carbon foam courtesy of GrafTech International, Ltd.)

Table 6.10. Yield Stress and Modulus Confidence Intervals for Test 20.3 of FPA-20

\begin{tabular}{|c|c|c|c|c|}
\hline & $\begin{array}{c}\text { Confidence } \\
\text { Interval }\end{array}$ & Mean & Error & $\%$ Error \\
\hline \multirow[t]{4}{*}{ Yield Stress (MPa) } & $95 \%$ & 16.5 & \pm 0.74 & 4.48 \\
\hline & $90 \%$ & 16.5 & $\pm \mathbf{0 . 5 7}$ & 3.45 \\
\hline & $80 \%$ & 16.5 & $\pm \mathbf{0 . 3 7}$ & 2.24 \\
\hline & $75 \%$ & 16.5 & $\pm \mathbf{0 . 3 0}$ & 1.81 \\
\hline \multirow[t]{4}{*}{ Modulus (MPa) } & $95 \%$ & 698 & \pm 27.1 & 3.88 \\
\hline & $90 \%$ & 698 & \pm 20.5 & 2.94 \\
\hline & $80 \%$ & 698 & \pm 13.2 & 1.89 \\
\hline & $75 \%$ & 698 & \pm 10.5 & 1.50 \\
\hline
\end{tabular}



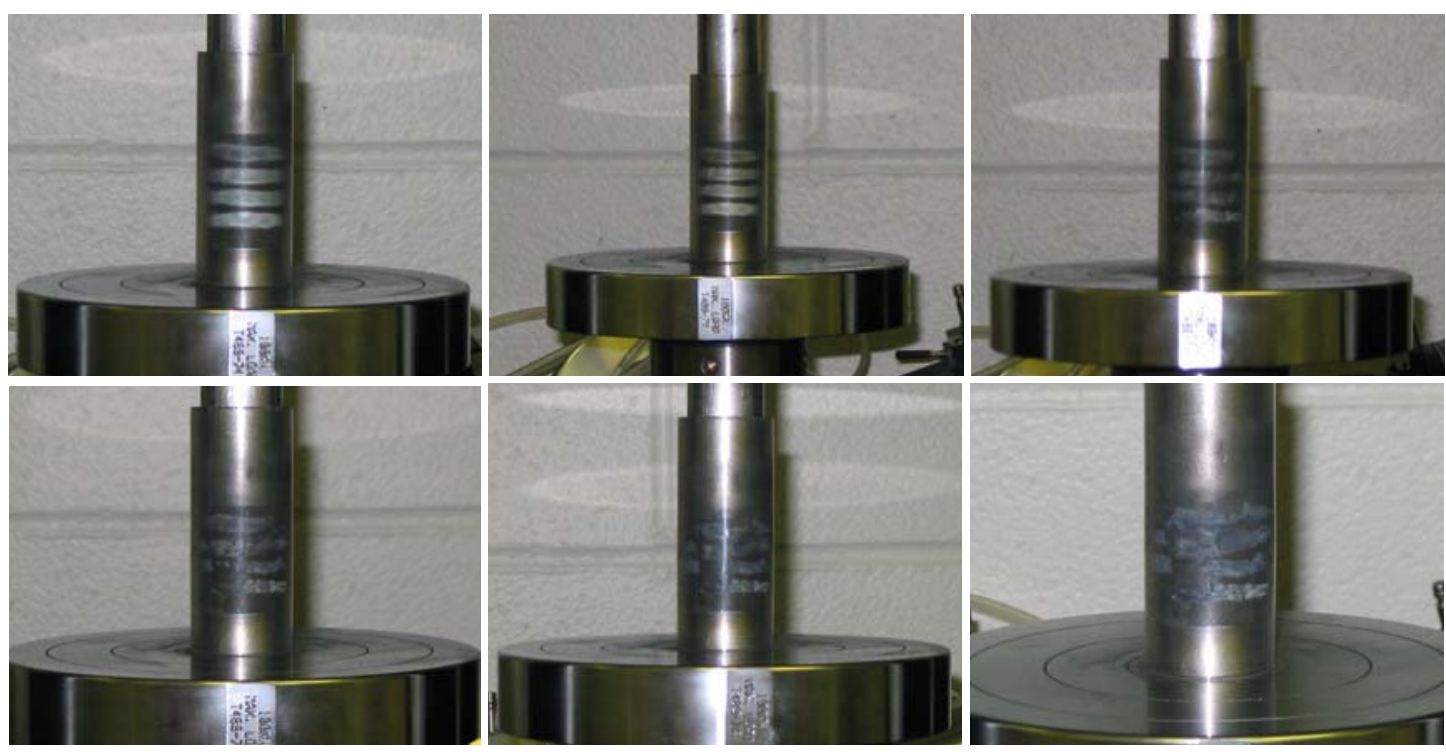

Figure 6.8. Photos taken at regular intervals (beginning at upper left) during a constrained compression test of FPA-20 carbon foam. (FPA-20 carbon foam courtesy of GrafTech International, Ltd.)

\subsection{Discussion on GrafTech Foams}

The three GrafTech foams were evaluated for compression behavior in order to perform statistical analysis and verify the operation of the constrained method of testing. The experimental protocol for the GrafTech foams was designed so as to gather as much data as possible while controlling certain parameters that could affect the testing results.

FPA-02 was used most frequently because it was the lightest, easiest foam to work with and because it was provided in the largest quantity. It is also noted that FPA02 fails in a manner different than FPA-10 or FPA-20. This failure mechanism allows for easier observation and comparison with the unconstrained test.

The constrained piston test and the unconstrained compression tests were performed to determine if the piston assembly influenced the values of the yield stress or modulus. The main reason for utilizing the piston assembly is to ensure a one- 
dimensional volume change over the entire compression of the carbon foam sample so that energy absorption values can be gathered. The piston assembly should not influence the yield stress or the modulus. Unconstrained tests easily give results for yield stress and modulus. However, the changing cross-section (as is the case with FPA-10 and FPA20) causes the stress calculations to be flawed after the initial failure occurs. Therefore, the area under the curve is meaningless if the cross-section of the sample changes and hence the absorption energy can not be determined. The yield stress and modulus for the constrained technique are compared to those from the unconstrained technique to ensure that the initial portion of the stress vs. strain plots is not altered by the chosen technique. The constrained piston technique is shown to be more beneficial since it has the added capability of gathering energy absorption data regardless of the failure mode of the sample.

FPA-02 is extremely beneficial in this study because its failure mechanism allows its cross-sectional area to remain constant even during unconstrained testing. Therefore, the energy absorption results from the unconstrained tests can be compared directly with the constrained results in addition to the comparisons of yield stress and modulus. Indeed the results herein indicate a very favorable agreement between the two techniques provided the sample fails in a well-defined manner.

\subsubsection{Discussion of FPA-02 Results}

The results from each set of compression tests for the FPA-02 foam were compared together to determine if the differences between constrained and unconstrained 
testing, sample height, and platen speed affected the results. The yield stress data between Tests 2.1 and 2.2 are nearly identical. This indicates that there is little to no effect on results between platen movement of $1 \mathrm{~mm} / \mathrm{min}$ and $2 \mathrm{~mm} / \mathrm{min}$. Therefore tests can be performed in half the time originally expected by setting the platen speed at $2 \mathrm{~mm} / \mathrm{min}$. The overall platen movement is still restricted to lower rates, however, in order to allow air to escape from the piston assembly without pressurizing the inside of the chamber. It is important to note that air resistance has no affect whatsoever on the tests at these low strain rates. Low platen speeds are maintained to eliminate any type of effects from dynamic compression and air resistance.

Yield stress numbers from Test 2.4 also show that the same results at $2 \mathrm{~mm} / \mathrm{min}$ can be achieved with the piston assembly. Test 2.5 results (Table 6.11 ) are actually closer than the mean values show. One particular sample in Test 2.5 provided a high value for yield stress, which drove the mean value above 1.40 MPa. Otherwise, yield stress results from Test 2.5 are extremely close to those from Tests 2.1, 2.2, and 2.4. Comparing these results to the confidence intervals in Table 6.3, most of the mean yield stress values from the 5 sets of tests fall within the $95 \%$ confidence interval for yield stress, and most of the energy absorption variances are better than the calculated $50 \%$ confidence interval. However, nearly all mean modulus values fall just outside the $95 \%$ confidence interval. (This observation is probably due to Test 2.5 having larger mean values.) The confidence interval for the yield stress claims there is a $95 \%$ chance that the yield stress of FPA-02 is between 0.137 and $0.147 \mathrm{MPa}(0.142 \pm 0.005 \mathrm{MPa})$. The modulus results and confidence interval suggest that the mean modulus values of Tests 2.1-2.5 have been shifted. 
Table 6.11. Results of Compression Test 2.5 of FPA-02

\begin{tabular}{|c|cccc|}
\hline Sample Number & $\begin{array}{c}\text { Measured Bulk } \\
\text { Density }\left(\mathbf{g} / \mathbf{c m}^{\mathbf{3}}\right)\end{array}$ & $\begin{array}{c}\text { Yield } \\
\text { Stress (MPa) }\end{array}$ & $\begin{array}{c}\text { Modulus } \\
(\mathbf{M P a})\end{array}$ & $\begin{array}{c}\text { Energy } \\
\text { Absorption }\left(\mathbf{M J} / \mathbf{m}^{\mathbf{3}}\right)\end{array}$ \\
\hline $\mathbf{2 . 5 . 1}$ & 0.024 & 0.227 & 3.45 & 0.161 \\
$\mathbf{2 . 5 . 2}$ & 0.025 & 0.206 & 3.18 & 0.153 \\
$\mathbf{2 . 5 . 3}$ & 0.024 & 0.124 & 1.60 & 0.124 \\
$\mathbf{2 . 5 . 4}$ & 0.024 & 0.134 & 2.82 & 0.127 \\
$\mathbf{2 . 5 . 5}$ & 0.025 & 0.140 & 2.24 & 0.133 \\
$\mathbf{2 . 5 . 6}$ & 0.024 & 0.159 & 3.02 & 0.142 \\
$\mathbf{2 . 5 . 7}$ & 0.024 & 0.140 & 4.87 & 0.125 \\
$\mathbf{2 . 5 . 8}$ & 0.024 & 0.127 & 4.07 & 0.118 \\
$\mathbf{2 . 5 . 9}$ & 0.025 & 0.130 & 4.21 & 0.124 \\
$\mathbf{2 . 5 . 1 0}$ & 0.025 & 0.138 & 5.44 & 0.137 \\
\hline Mean & $\mathbf{0 . 0 2 4}$ & $\mathbf{0 . 1 5 3}$ & $\mathbf{3 . 4 9}$ & $\mathbf{0 . 1 3 4}$ \\
Std Dev & $\mathbf{0 . 0 0 0}$ & $\mathbf{0 . 0 3 5}$ & $\mathbf{1 . 1 8}$ & $\mathbf{0 . 0 1 4}$ \\
Variance & $\mathbf{0 . 0 0 0}$ & $\mathbf{0 . 0 0 1}$ & $\mathbf{1 . 3 9}$ & $\mathbf{0 . 0 0 0}$ \\
\hline
\end{tabular}

A closer comparison of specific data sets can give a better idea on how variables may affect the test. Comparison of the data suggests that the piston assembly does not increase the yield stress nor the modulus, but it may slightly increase the energy absorption. The height difference between 1 and 2 inches $(2.54$ and $5.08 \mathrm{~cm})$ in Tests 2.4 and 2.5 appears to affect all three parameters, inflating the results for yield stress $(0.140$ to $0.153 \mathrm{MPa}$ ), modulus (2.56 to $3.49 \mathrm{MPa})$, and energy absorption $(0.122$ to 0.134 $\mathrm{MJ} / \mathrm{m}^{3}$ ) when the sample height is increased to 2 inches. However, considering the fact that Sample 2.5.1 provided a yield stress result that was higher than all others in the FPA02 series of tests, this observed difference in the mean values of Test 2.5 might not be significant. Although the differences in the mean values may not be real, it is significant that Test 2.5 has the largest standard deviations for yield stress, modulus, and energy absorption (regardless of the values provided by Sample 2.5.1). This suggests that the 2inch tall samples have the largest range of values, and thus are less repeatable than the 
other four tests. Finally, the platen speed does not seem to have a significant affect on any of the measured quantities. The platen speed was limited to $2 \mathrm{~mm} / \mathrm{min}$ in order to ensure than no air resistance would be a factor as air escapes from the sample chamber between the piston and the cylinder. The data presented in Table 6.2 suggest that Test 2.4 provides results that have the lowest standard deviation for modulus, a yield stress result that falls within the $95 \%$ confidence interval, and a fairly low variance for energy absorption with an average value very close to the rough population mean.

\subsubsection{Discussion of FPA-10 Results}

A comparison of Test 10.1 and Test 10.2 indicates that the piston assembly has no affect on the yield stress or modulus. In Test 10.2 the yield stress and modulus are larger than in the unconstrained test. However, these values for Test 10.3 are lower. The mean bulk density of the samples in Test 10.3 was actually a bit lower than the bulk densities in Tests 10.1 and 10.2. This explains why the results are the lowest. Despite this finding, the yield stress results are still very close to each other suggesting that the piston has little to no effect on the results of the compression tests.

Looking at confidence intervals, the range of the yield stress is quite good. In the $95 \%$ confidence interval, the error of nearly $\pm 1.5 \%$ is the best of any other value in any set of data. The individual sample standard deviations for each set of samples are low and are very comparable to that of the unbiased standard deviation calculated for all combined data. However, the range of the modulus confidence interval is a little larger at just over $\pm 4.5 \%$, and the standard deviations are higher than anticipated. This suggests 
that the range of modulus readings has the highest spread compared to the results of yield stress and energy absorption. This problem can be the result of operator error, sample preparation, or equipment failure and will be addressed in the next section.

\subsubsection{Discussion of FPA-20 Results}

FPA-20 was tested in the same way as FPA-02 and FPA-10 in the fact that constrained and unconstrained results were compared for yield stress and modulus. Again, between the three sets of tests, there seems to be little to no effect from the piston assembly on the yield stress values. However, after Test 20.2, the sample standard deviations of the modulus data were, again, quite high. Noticing that the modulus standard deviations were becoming larger and larger with the increase in density of the foams, an effort was made to gather results with a lower standard deviation. Since a similar trend was not observed from the yield stress or energy absorption, it was assumed that the problem was not due to equipment issues or the testing technique itself. Therefore, sample preparation issues were addressed as a possible cause for the large spread of modulus results.

Test 20.3 is the result of that effort. It was discovered that modulus is highly dependant on the surface structure of the samples being compressed. All previous samples were cut using stationary power tools (drill press, band-saw, etc.) with the best precision possible at the time. However, it was noted that the surfaces in contact with the compression device (whether the piston assembly or the platens directly) were not always parallel. Therefore, the faces of the samples were not always in full contact with the 
faces of the compression devices. Since the modulus is calculated using the part of the stress vs. strain plot before the sample fails, the initial dimensions and shape of the sample greatly influence the results. Thus, Test 20.3 was performed with the sole purpose of investigating the effects of sample preparation and reducing the standard deviation of the modulus.

Samples for Test 20.3 were cut out using the same 1.25-in OD hole-saw as all other samples. However, the ends were cut with the aid of a jig that was not previously available. This jig was clamped to the band-saw's table and used as a straightedge guide to allow all 10 samples to be cut identically with better precision. During the previous tests, samples were cut as precisely as possible by hand without the help of a guide. However, this test shows that the use of the guide greatly improves the repeatability of the results, and lowers the scatter of the data.

This test was performed successfully. The standard deviation of the yield stress was reduced from 3.9 down to 1.3 , and more impressively, the standard deviation of the modulus was reduced from over 136 down to just below 45 . This result shows that more precise machining of the sample can reduce these deviations, giving results that are very reproducible as has been shown by the difference between Test 20.2 and Test 20.3.

The photos from the constrained test were taken for observation. The stainless steel cylinder prohibits visual observation of the samples during compression and so very little was known about the physical interaction between the piston and the sample contained within the assembly during such a test. Therefore, the Lexan ${ }^{\circledR}$ cylinder was employed to allow such an observation. The photos using the Lexan ${ }^{\circledR}$ cylinder show that the piston remains in constant contact with the sample surface. They also show that the 
piston assembly does not prohibit the samples from failing normally as shown in the unconstrained testing photos, yet it does prohibit the sample's cross-sectional area from changing. Figure 6.7 shows that the foam still cracks through the middle, as it does during the unconstrained test (Figure 6.6). However, instead of breaking and falling apart into several pieces, the foam's initial cross-section is retained. It can then be concluded that the piston does not negatively interfere with the failure mechanism of the sample during the compression test, while keeping the cross-section of the sample constant. Maintaining a constant cross-section throughout the compression test allows meaningful energy absorption result to be attained.

\subsection{MER, WVU, and Ultramet Foam Results}

The purpose of testing this group of foams is that these foams are very different from the GrafTech series. The MER foam and WVU foam are denser than FPA-20, and the Ultramet foam has a much different internal structure than any of the other foams. There was not enough material to perform statistical analysis on any of these foams, other than a mean value with standard deviation. There was not enough material to make any type of changes to the tests or samples after the initial tests were performed, so the standard deviations are not as low as those from the GrafTech series. In spite of this fact, this series of tests was still successful because the main goal of them was to show that the compression test described here can successfully be performed on any foam with any density. 


\subsubsection{MER Foam Results}

The tests on MER foam (structure shown in Figure 6.9) were performed because it was the foam with the highest density tested for this work measured at $37-1 \mathrm{~b} / \mathrm{ft}^{3}(0.59$ $\mathrm{g} / \mathrm{cm}^{3}$ ). The main intent was to show that foams of a higher density than FPA-20 or foams with different bulk structure could be successfully tested using this method. There was very little material so only two sets of three samples were run for this particular study. However, successful tests did return results for yield stress, modulus, and energy absorption (for the constrained technique only). The tests performed are described in Table 6.12 and the results are shown in Table 6.13 .

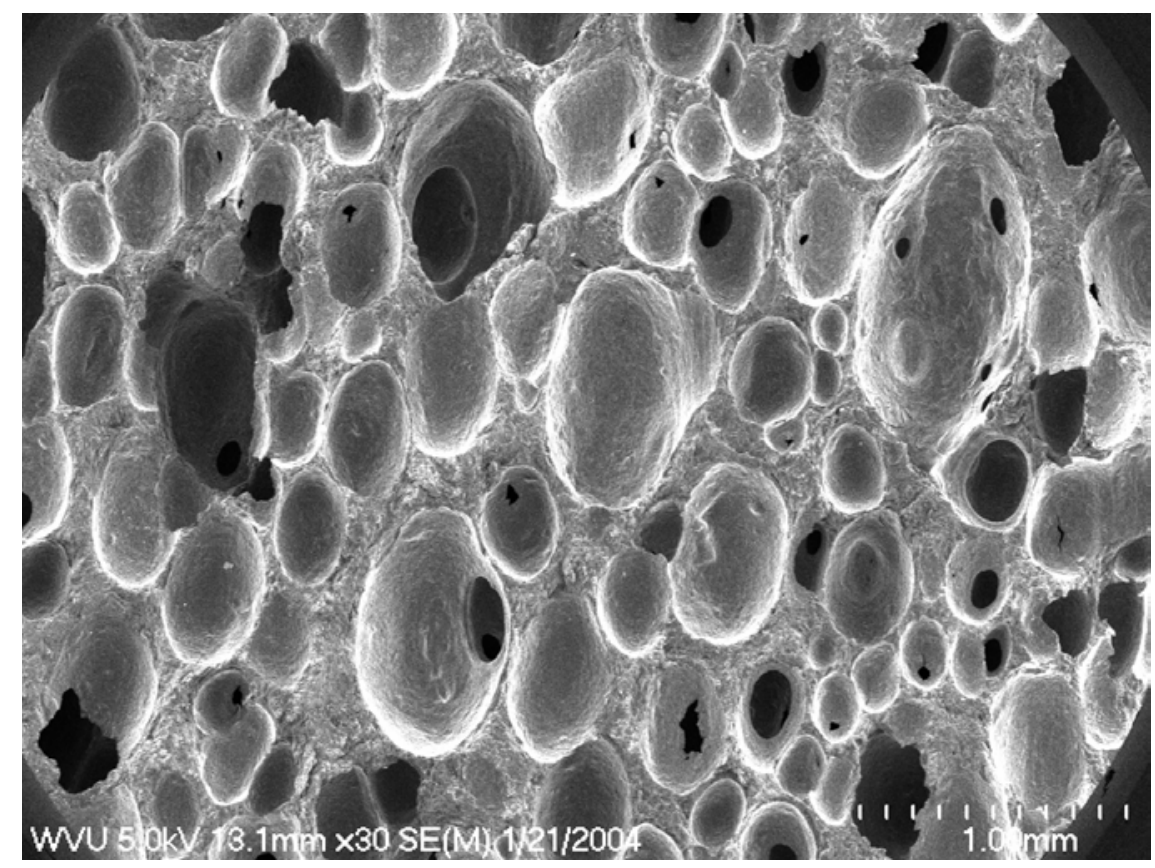

Figure 6.9. SEM Micrograph of Carbon Foam from MER Corporation. 
Table 6.12. Parameters for Compression Tests of Carbon Foam from MER Corp.

\begin{tabular}{|l|ccc|}
\hline & $\begin{array}{c}\text { Test } \\
\text { Type }\end{array}$ & $\begin{array}{c}\text { Sample } \\
\text { Height }(\mathbf{c m})\end{array}$ & $\begin{array}{c}\text { Platen Speed } \\
(\mathbf{m m} / \mathbf{m i n})\end{array}$ \\
\hline Test MER.1 & Unconstrained & 2.54 & 1 \\
Test MER.2 & Constrained & 2.54 & 1 \\
\hline
\end{tabular}

Table 6.13. Mean Results from Each Set of Compression Tests for MER Foam

\begin{tabular}{|r|cccc|}
\hline & $\begin{array}{c}\text { Measured Bulk } \\
\text { Density }\left(\mathbf{g} / \mathbf{c m}^{3}\right)\end{array}$ & $\begin{array}{c}\text { Yield } \\
\text { Stress }(\mathbf{M P a})\end{array}$ & $\begin{array}{c}\text { Modulus } \\
(\mathbf{M P a})\end{array}$ & $\begin{array}{c}\text { Energy } \\
\text { Absorption }\left(\mathbf{M J} / \mathbf{m}^{3}\right)\end{array}$ \\
\hline Test MER.1 & $\mathbf{0 . 5 9 1}$ & $\mathbf{2 2 . 8}$ & $\mathbf{1 1 6 0}$ & $\mathbf{n a}$ \\
Std Dev. & & 3.4 & 522 & na \\
Variance & & 11.5 & 272484 & na \\
\hline Test MER.2 & $\mathbf{0 . 5 9 6}$ & $\mathbf{2 0 . 8}$ & $\mathbf{9 1 0}$ & $\mathbf{1 2 . 3}$ \\
Std Dev. & & 2.0 & 20 & 0.5 \\
Variance & & 4.1 & 400 & 0.3 \\
\hline
\end{tabular}

\subsubsection{WVU Foam Results}

The foam produced at West Virginia University (Figure 6.10) is a coal-based carbon foam, so the foam in general is different from the others with respect to the bulk matrix, precursor material, and the manufacturing process. The foam from WVU also has a larger bulk density of $34 \mathrm{lb} / \mathrm{ft}^{3}\left(0.54 \mathrm{~g} / \mathrm{cm}^{3}\right)$ than those foams from GrafTech. Therefore, because of these differences, the foam from WVU was tested, much like the MER foam, to show that this technique can successfully be used on any type of carbon foam, regardless of bulk density, precursor material, or manufacturing process. The tests are outlined in Table 6.14 and results are shown in Table 6.15. The initial foam piece, like that of FPA-20, was not 2 inches in height initially. Therefore, sample heights of 1.5 inches $(3.81 \mathrm{~cm})$ were tested instead of 2-inch samples. 


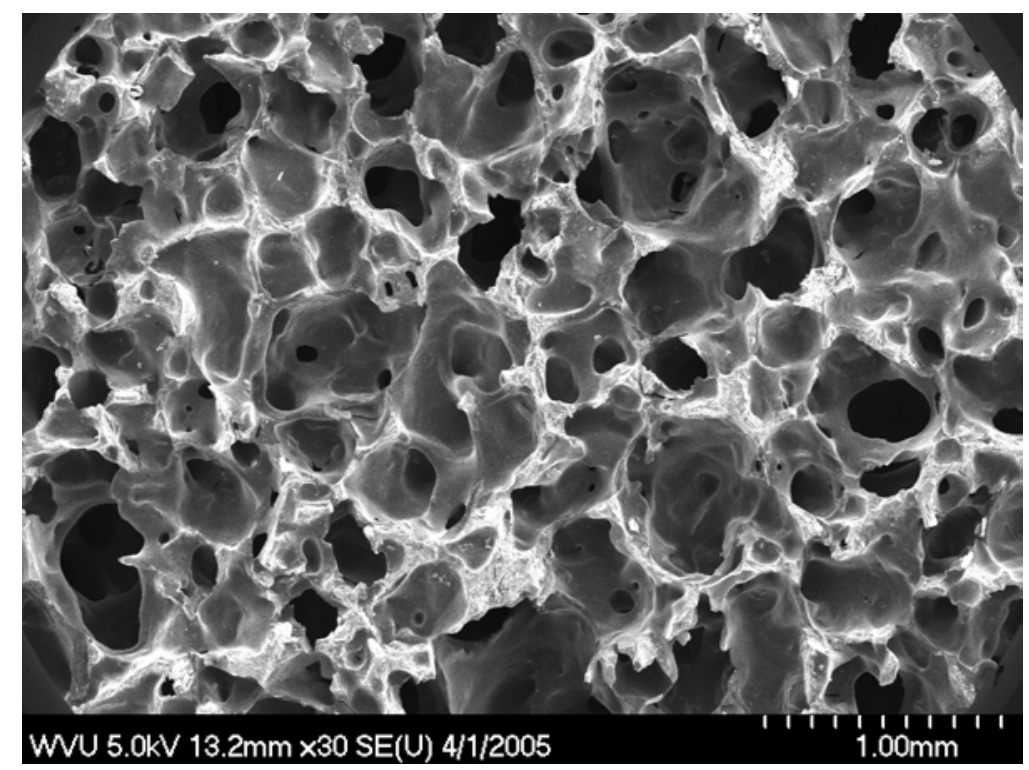

Figure 6.10. SEM Micrograph of Carbon Foam from WVU.

Table 6.14. Parameters for Compression Tests of Carbon Foam from WVU

\begin{tabular}{|l|ccc|}
\hline & $\begin{array}{c}\text { Test } \\
\text { Type }\end{array}$ & $\begin{array}{c}\text { Sample } \\
\text { Height }(\mathbf{c m})\end{array}$ & $\begin{array}{c}\text { Platen Speed } \\
(\mathbf{m m} / \mathbf{m i n})\end{array}$ \\
\hline Test WVU.1 & Unconstrained & 3.81 & 1 \\
Test WVU.2 & Constrained & 3.81 & 1 \\
\hline
\end{tabular}

Table 6.15. Mean Results from Each Set of Compression Tests for WVU Foam

\begin{tabular}{|r|cccc|}
\hline & $\begin{array}{c}\text { Measured Bulk } \\
\text { Density }\left(\mathbf{g} / \mathbf{c m}^{\mathbf{3}}\right)\end{array}$ & $\begin{array}{c}\text { Yield } \\
\text { Stress }(\mathbf{M P a})\end{array}$ & $\begin{array}{c}\text { Modulus } \\
(\mathbf{M P a})\end{array}$ & $\begin{array}{c}\text { Energy } \\
\left.\text { Absorption } \mathbf{( M J} / \mathbf{m}^{\mathbf{3}}\right)\end{array}$ \\
\hline Test WVU.1 & $\mathbf{0 . 5 5 8}$ & $\mathbf{8 . 8 8}$ & $\mathbf{3 4 7}$ & $\mathbf{\text { na }}$ \\
Std Dev. & & 0.14 & 36 & na \\
Variance & & 0.02 & 1296 & na \\
\hline Test WVU.2 & $\mathbf{0 . 5 3 6}$ & $\mathbf{6 . 0 8}$ & $\mathbf{3 5 0}$ & $\mathbf{6 . 0 8}$ \\
Std Dev. & & 0.36 & 49 & 0.36 \\
Variance & & 0.13 & 2401 & 0.13 \\
\hline
\end{tabular}




\subsubsection{Ultramet Foam Results}

A foam from Ultramet (shown in Figure 6.11) was also evaluated. This particular foam fails much like FPA-02. The cross-sectional area of this lightweight foam (3.87 $\mathrm{lb} / \mathrm{ft}^{3}, 0.062 \mathrm{~g} / \mathrm{cm}^{3}$ ) does not change during compression, which allows energy absorption to be calculated for both constrained and unconstrained tests. The amount of material allowed for more sets of tests to be performed, but since there were only five samples per test, statistical analysis was not performed. However, the standard deviation and the mean were calculated for all the tests to compare differences in sample height and to observe any differences that the piston assembly may have had on the compression results. The tests are outlined in Table 6.16 and the results are shown in Table 6.17.

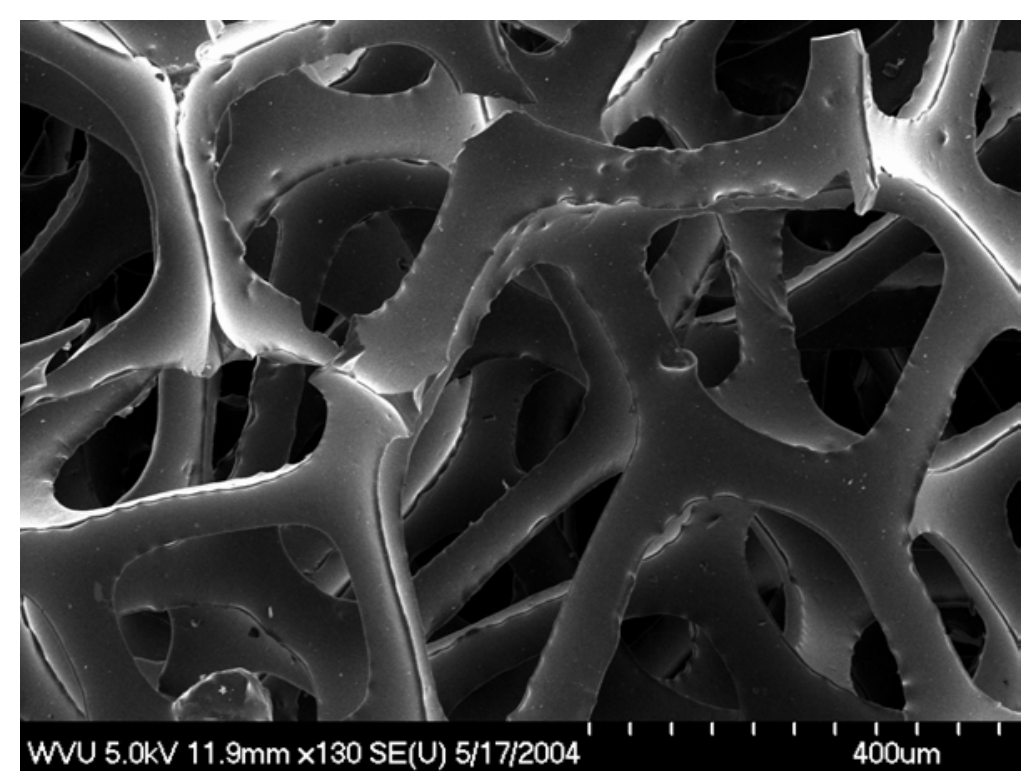

Figure 6.11. SEM micrograph of Carbon Foam from Ultramet. 
Table 6.16. Parameters for Compression Tests of Carbon Foam from Ultramet

\begin{tabular}{|l|ccc|}
\hline & $\begin{array}{c}\text { Test } \\
\text { Type }\end{array}$ & $\begin{array}{c}\text { Sample } \\
\text { Height }(\mathbf{c m})\end{array}$ & $\begin{array}{c}\text { Platen Speed } \\
(\mathbf{m m} / \mathbf{m i n})\end{array}$ \\
\hline Test U.1 & Unconstrained & 5.08 & 1 \\
Test U.2 & Unconstrained & 2.54 & 1 \\
Test U.3 & Constrained & 5.08 & 1 \\
Test U.4 & Constrained & 2.54 & 1 \\
\hline
\end{tabular}

Table 6.17. Mean Results from Each Set of Compression Tests for Ultramet Foam

\begin{tabular}{|c|c|c|c|c|c|}
\hline & & $\begin{array}{l}\text { Measured Bulk } \\
\text { Density }\left(\mathrm{g} / \mathrm{cm}^{3}\right)\end{array}$ & $\begin{array}{c}\text { Yield } \\
\text { Stress (MPa) }\end{array}$ & $\begin{array}{c}\text { Modulus } \\
\text { (MPa) }\end{array}$ & $\begin{array}{c}\text { Energy } \\
\text { Absorption }\left(\mathrm{MJ} / \mathrm{m}^{3}\right)\end{array}$ \\
\hline \multirow[t]{3}{*}{ Test U.1 } & & - & 0.316 & 31.5 & 0.222 \\
\hline & Std Dev. & & 0.021 & 2.81 & 0.040 \\
\hline & Variance & & 0.000 & 7.90 & 0.002 \\
\hline \multirow[t]{3}{*}{ Test U.2 } & & 0.062 & 0.342 & 21.0 & 0.255 \\
\hline & Std Dev. & & 0.058 & 8.31 & 0.057 \\
\hline & Variance & & 0.003 & 69.1 & 0.003 \\
\hline \multirow[t]{3}{*}{ Test U.3 } & & 0.052 & 0.351 & 28.8 & 0.269 \\
\hline & Std Dev. & & 0.033 & 2.77 & 0.071 \\
\hline & Variance & & 0.001 & 7.67 & 0.005 \\
\hline \multirow[t]{3}{*}{ Test U.4 } & & 0.063 & 0.344 & 14.3 & 0.306 \\
\hline & Std Dev. & & 0.019 & 1.99 & 0.040 \\
\hline & Variance & & 0.000 & 3.96 & 0.002 \\
\hline
\end{tabular}

\subsection{Discussion of MER, WVU, and Ultramet Foam Results}

These foams were tested to show that the constrained piston compression test developed herein could successfully be performed on any carbon foam of any bulk density, precursor material, or manufacturing process. The small amount of material from each foam limited the number of samples and therefore statistical analysis could not be performed. However, the mean values for the constrained and unconstrained tests for each individual foam were compared. Despite the small number of samples, the results 
for yield stress are relatively close together regardless of constrained or unconstrained testing and the associated standard deviations are fairly low.

\subsubsection{Discussion of MER Foam Results}

The results from MER foam were surprisingly close considering only six total samples were tested. Figure 6.9 shows the anisotropy of the matrix of the foam, with elongated cells of different dimensions. Despite this fact, the results were still quite close to each other. The direct effect of anisotropy of the cell shapes was not part of the focus of this study and thus, was not investigated. The foam community, in general, agrees that foams are strongest in the foaming, or rise, direction (the direction of the cell elongation) and so all of these foams were tested in this direction.

The piston assembly does not seem to have any effect as far as altering the values of the yield stress and modulus. It is important to note that the relation between modulus and sample preparation (as described for Test 20.3) was made after these tests (as well as Ultramet and WVU) were performed. The initial sample heights were just below 1 inch and it was quite difficult and unsafe to handle the samples so close to the saw-blade in order to cut the edges and ensure they were parallel. Therefore the large standard deviations of the unconstrained test are most likely due to poor sample preparation, not the actual compression test itself. In addition, only three samples were available for each set of tests, which also will increase standard deviation. However, the constrained technique successfully gathered yield stress values that were in good agreement with those of the unconstrained test, the cluster of results for the modulus is much closer for 
the constrained test, and energy absorption was successfully calculated with a standard deviation below 0.5 .

\subsubsection{Discussion of WVU Foam Results}

The foam from West Virginia University is similar to MER in that the amount of data that could be gathered was small and yet the results were extremely close to each other. The average values of yield stress and modulus changed very little between the constrained and unconstrained test. Due to the small amount of data gathered, it is not possible to say with any certainty that the piston assembly added any resistance to cause the constrained values to increase slightly. In fact, the data from all other foams vary slightly up and down, seemingly independent of whether or not the test was constrained or unconstrained.

\subsubsection{Discussion of Ultramet Foam Results}

The foam from Ultramet behaved very much like FPA-02 in the sense that energy absorption could be gathered using both the unconstrained test as well as the piston assembly. This type of failure allows the energy absorption values of the constrained tests to be compared directly to those from the unconstrained tests. The same is true for the yield stress and modulus so that any effect of the piston assembly can be observed. The difference between 1 and 2 inch sample heights does not seem to affect the results. All yield stress and energy absorption values are nearly within standard deviation limits 
of each other which implies that value differences are not significant. It does appear that the constrained test increases all of the results. However, when this trend is compared to the other tests (from GrafTech, WVU, MER foams), there does not seem to be any similar tendencies to support this theory. That is to say, none of the other results from this study seems to provide adequate evidence to suggest that the constrained technique influences the testing results. Since there were only five samples per set of tests, it is difficult to make any absolute conclusions in this regard.

\subsection{Observations from Results}

Based on the results from all of the compressive tests performed in this study, a few general observations can be made. One observation is based on the density relation with yield stress. Studying the SEM micrographs and comparing them with the bulk densities of the foams, it is apparent that, in general, if a foam has a higher bulk density, the yield stress, modulus, and energy absorption are higher. This is as expected because the higher the density of the foam, the more solid material it will contain. It was also observed that density is not the only determining factor for foam strength. WVU foam is nearly twice as dense as FPA-20, but the yield stress results are higher for FPA-20. The SEM micrographs show that the two foams have vastly different structure. For example, the cell sizes are different, the distribution of the cell sizes is different, and the cell walls have different thicknesses. Hence, the physical structure of the foam also has some effect on foam strength. 
Several further observations can be made from the test results. The data show that the speed of the platen can be increased from 1 to $2 \mathrm{~mm} / \mathrm{min}$. The speed of the test is kept low for a few reasons. Dynamic crushing drives the resulting yield stress upwards due to localization of deformation and densification. These results drive up local strain rates and do not allow force to be evenly displaced over the entire volume of the sample. Other speeds were not evaluated (such as $3 \mathrm{~mm} / \mathrm{min}$ or $5 \mathrm{~mm} / \mathrm{min}$ ) due to an overall lack of material. Testing was limited to 1 and $2 \mathrm{~mm} / \mathrm{min}$ in order to gain more data from these two speeds so that useful comparisons could be made. Another reason is to avoid completely any influence on results from air resistance as air escapes the piston assembly. It is observed that the weight of the piston pushes out the air from the sample chamber if there is no foam sample in the piston assembly. The piston falls much faster than $2 \mathrm{~mm} / \mathrm{min}$. Therefore, it is determined that, in the platen speed range of 1 to $2 \mathrm{~mm} / \mathrm{min}$, air resistance is not a factor in the constrained piston assembly compression test.

The piston itself may also have its own influence on the values of the tests. The piston weighs $343 \mathrm{~g}(0.756 \mathrm{lb})$ and has a cross-sectional area of $79.7 \mathrm{~mm}^{2}\left(0.124 \mathrm{in}^{2}\right)$. This results in an additional stress of $0.04 \mathrm{MPa}$ on the testing values. Since this value is so small compared to the yield stresses for most of the foams tested (FPA-10, FPA-20, MER, WVU), it has been ignored. It probably has some effect on FPA-02 and the Ultramet foam, however, none of the recorded differences between constrained and unconstrained (with and without piston) are 0.04 MPa. For example, the yield stress for Test U.2 (unconstrained) is $0.342 \mathrm{MPa}$ while the value for Test U.4 (constrained) is 0.344 $\mathrm{MPa}$, a difference of $0.002 \mathrm{MPa}$. Therefore, any stress addition due to the weight of the piston was ignored for these foams. 
The data also allowed comparison of 1, 1.5, and 2-inch $(2.54,3.81$, and $5.08 \mathrm{~cm})$ sample height. The results, in general, show that 1-inch tall samples yield results with much lower standard deviation than the 2-inch tall samples. If more material is trapped within the piston assembly during testing, there is a better chance that dust and particles will be available to interfere with the interaction between the piston face and the sample itself. Therefore, a 1-inch tall sample is preferred. No samples below 1 inch were evaluated because all current ASTM compression methods require samples with a height to diameter ratio of at least 1.0 .

Most importantly, the constrained tests provided measurements that were entirely consistent with those from the unconstrained test. There was very little to no statistically significant difference between any constrained or unconstrained test in any of the testing groups (FPA-02, FPA-10, MER, WVU, etc.). The yield stress and modulus values were all very comparable as expected, and the energy absorption data for FPA-02 and Ultramet showed very little variation between the two testing techniques. This suggests that the constrained technique is a more preferred way to gather yield stress, modulus, and volumetric energy absorption data in one test.

Due to the anisotropic structure of the foams, it is expected that there will be error in the results of any set of tests. No two pieces of the same foam will yield the exact same results because these foams do not have identical perfect structures. Therefore, differences in mean values, standard deviations, and confidence intervals are expected. The 95\% confidence intervals calculated for the three GrafTech samples have errors as low as $1.5 \%$ up to about $7.5 \%$. However with the completion of Test 20.3 (of FPA-20), it has been shown that the error can be reduced with more precise sample preparation. It is 
imperative that the sample surfaces are parallel to each other and to the compression faces. These surfaces must also be perpendicular to the sides to ensure that the forces applied to the sample are kept at a $90^{\circ}$ angle to the compression faces. Facing the specimens on a lathe or a surface grinder is recommended if it is possible to hold them firmly in the chuck without crushing them.

Currently, there are no theoretical or other experimental values to which the results of these tests can be compared. For this reason, the standard deviation of the data and the percent error of the confidence intervals are even more important to display the precision of this testing method. It has been shown herein that the newly-proposed constrained technique can consistently return reproducible results that are not only comparable to the conventional unconstrained technique, but also allow the energy absorption data to be obtained in the same test. 


\section{Chapter 7}

\section{Summary and Conclusions}

Several types of carbon foam were successfully tested using the proposed constrained piston method for compression of carbon foams. In addition to validating the technique for a wide variety of foam, several other parameters were investigated, including the platen speed (increased from 1 to $2 \mathrm{~mm} / \mathrm{min}$ ), and the defined sample height of 1 inch $(2.54 \mathrm{~cm})$. Statistical analysis was performed on the data from FPA-02, FPA-10, and FPA-20 in order to gather confidence intervals and some indication of the precision of the technique.

It was noted that improved sample preparation in the machining and measurement of the samples decreases the error in the confidence interval and the standard deviation of the data. Therefore it is imperative that the faces of the sample be parallel to each other and parallel to the face of the piston assembly. The entire area of the samples must be in complete contact with the compression faces of the piston assembly throughout the duration of the compression test. These faces must also be perpendicular to the circular sides in order to make sure that the forces exerted on the samples are not being diverted away from the center of the samples.

Finally, the technique was successfully demonstrated on carbon foams from Ultramet, MER, and WVU. These foams have various densities up to two times that of FPA-20. Statistical analysis was not performed on these foams due to the small number of samples that were available for testing.

This study has shown that the constrained piston technique can successfully be used to gather compression data from carbon foams. Yield stress, modulus, and energy 
absorption can all be obtained in one testing session. Thus it is a distinct improvement over the conventional unconstrained tests in which energy absorption data may not always be accessible. Finally, it has been shown that the constrained test gives nearly identical values of both yield stress and modulus compared to those from the unconstrained test. 


\section{Chapter 8}

\section{Recommendations}

These tests were designed to gather statistical analysis and information on the proposed constrained compression testing method for carbon foam. In order to gather the most reliable data with the smallest confidence intervals, more samples (well over 100) of each individual foam specimen should be tested.

Compression studies should be done in order to compare foam strength in other directions other than the foaming, or rise, direction. Although the foam community agrees that the foaming direction is the strongest, the effects on strength from the foam orientation within the compression test should be investigated.

Different materials should be used to construct the piston used in the constrained method to determine it any effects caused by the weight of the piston can be measured or identified.

Theoretical studies should be done on the foams tested in this study. There are no ideal, or preferred values at this time with which to compare absolute values of yield stress, modulus, or energy absorption. Therefore, modeling should be done in order to gain mathematical information with which to compare the experimental values. Moreover, where possible, experimental data from other researchers should be obtained on these same foams (i.e. GrafTech foams) so the results herein can be compared with data generated by an independent unbiased laboratory. 
It is recommended that all carbon foams be tested using the following proposed compression test method.

\subsection{Test Specimen}

Specimens shall be a right cylinder. Great care must be taken to insure that the faces of the right cylinder are parallel to each other and perpendicular to the cylindrical sides. The diameter of each cylinder shall be $25 \mathrm{~mm}$ and the height shall be $25 \mathrm{~mm}$ ( 1 inch). The sample are to be prepared such that compression is performed in the rise direction of the foam. That is, any cell elongation that may be present within the foam matrix should be aligned in the direction of compression.

\subsection{Sample Preparation}

The specimens should be conditioned for 24 hours in the room in which the test is performed.

\subsection{Testing Apparatus}

Compression tests on the carbon foam have been successfully performed using a load frame like the Instron 5869 fitted with a $50 \mathrm{kN}$ load cell (any other comparablyequipped apparatus should be suitable). In order to control the sample cross-section during compression testing, a piston assembly has been constructed out of stainless steel. 
A hollow, stainless steel cylinder has been turned on a lathe with an inside diameter of $25.42 \mathrm{~mm} \pm 0.0254 \mathrm{~mm}(1.001 \pm 0.001 \mathrm{in})$. An $88.9 \mathrm{~mm}$ (3.50in) long solid plunger and a $12.7 \mathrm{~mm}(0.50 \mathrm{in})$ long solid plug were also constructed with an outside diameter of $25.37 \mathrm{~mm} \pm 0.0254 \mathrm{~mm}(0.999 \pm 0.001 \mathrm{in})$. The plug is employed as a false bottom for the sample chamber cavity and provides a stable base for the specimen, while allowing easy removal of the crushed specimen. The foam is compressed between the solid plunger and the solid plug to ensure a one-dimensional volume change between the two parallel surfaces.

\subsection{Procedure}

The height and diameter of each specimen are recorded as an average of 3 different measurements using a caliper to the nearest \pm 0.001 in $( \pm 0.0254 \mathrm{~mm})$. The measurement shall be taken when the faces of the caliper first come into contact with the specimen and there should be no force from the caliper applied to the specimen. One diameter measurement is taken across the middle of cylinder, and the other two measurements are taken on opposite ends of the specimen. The height measurements are taken similarly, one across the center of the cylinder face, and two at opposite ends. The specimen weight $( \pm 0.01 \mathrm{~g})$ shall also be taken so that bulk density can be calculated. The specimen shall be placed in the piston assembly, assuring that the loaded ends are parallel and no binding can occur between the piston and the cylinder. The piston assembly is placed between the platens, being sure that the assembly is centered on the lower platen. The platens shall compress at no greater than $2 \mathrm{~mm} / \mathrm{min}$ to assure that there is no air 
resistance being recorded as air escapes the cylinder. Stress vs. strain data are taken until the densification region is reached, whereupon the test is terminated.

\subsection{Calculations}

The yield strength (or compressive strength) $C(\mathrm{MPa})$ is defined as the maximum load $F_{m}(\mathrm{~N})$ of the initial linear portion of the stress-strain curve divided by the apparent cross-sectional area $A_{c}\left(\mathrm{~mm}^{2}\right)$ of the foam sample being tested.

$$
C=\frac{F_{m}}{A_{c}}
$$

The Young's Modulus $E_{c}(\mathrm{MPa})$ is the slope of the linear portion of the plot and is defined as the change in stress divided by the change in strain.

$$
E_{c}=\frac{y_{1}-y_{2}}{\left(\frac{x_{1}-x_{2}}{100}\right)}
$$

where $x_{1}, y_{1}$ is a set of data for strain (\% strain) and stress (MPa) at an instant, and $x_{2}, y_{2}$ is a set of data for strain and stress at another instant (both are on the linear portion of the curve). A straight-edge against the linear portion of the stress-strain plot in order to assist in finding the correct $x$ and $y$ values. 
The total energy absorbed per unit volume is found by integrating the area under the Stress vs. Strain plot. Integration can be carried out using the Newton-Cotes closed integration formula where:

$$
\begin{gathered}
x_{i}=\frac{x_{n+1}-x_{n}}{100} \\
y_{i}=\frac{y_{n}+y_{n+1}}{2} \\
A=\sum_{1}^{i} x_{i} y_{i}
\end{gathered}
$$

The total area $A$ under the stress-strain plot, which is the total energy absorbed by the foam, is calculated by the summation of the products of each individual piece of area. The individual pieces are defined as the product of $x_{i}$ and $y_{i}$. The term, $x_{i}$, is defined as the difference between two consecutive data points of strain $(\%)$, where the $y_{i}$ term is the arithmetic mean of two consecutive data points of stress (MPa). The area under the plot is the total energy absorbed per unit volume expressed in $\mathrm{MJ} / \mathrm{m}^{3}$. Dividing the total energy by the sample density results in specific energy $(\mathrm{kJ} / \mathrm{kg})$. 


\section{Bibliography}

1) Gibson, Lorna J. and Ashby, Michael F., Cellular Solids Structure and Properties $2^{\text {nd }}$ ed. Cambridge University Press, 1997; Chapter 5

2) Gibson, Lorna J. and Ashby, Michael F., Cellular Solids Structure and Properties $2^{\text {nd }}$ ed. Cambridge University Press, 1997; Chapter 6

3) Gibson, Lorna J. and Ashby, Michael F., Cellular Solids Structure and Properties $2^{\text {nd }}$ ed. Cambridge University Press, 1997; Chapter 8

4) ASTM C67-99a, Standard Test Methods for Sampling and Testing Brick and Structural Clay Tile. Annual Book of ASTM Standards, 2000; Section 4 Construction Vol. 04.05

5) ASTM C695-91(95), Standard Test Method for Compressive Strength of Carbon and Graphite. Annual Book of ASTM Standards, 2000; Section 5 Petroleum Products, Lubricants, and Fossil Fuels Vol. 05.05

6) ASTM D1621-00, Standard Test Method for Compressive Properties of Rigid Cellular Plastics. Annual Book of ASTM Standards, 2000; Section 8 Plastics Vol. 08.01

7) ASTM D695-96, Standard Test Method for Compressive Properties of Rigid Plastics. Annual Book of ASTM Standards, 2000; Section 8 Plastics Vol. 08.01

8) ASTM F414-96, Standard Test Method for Energy Absorbed by a Tire When Deformed by a Slow-Moving Plunger. Annual Book of ASTM Standards, 2000; Section 9 Rubber Vol. 09.02 


\section{Appendix A}

\section{Stress vs. Strain Plots}


2.1.1

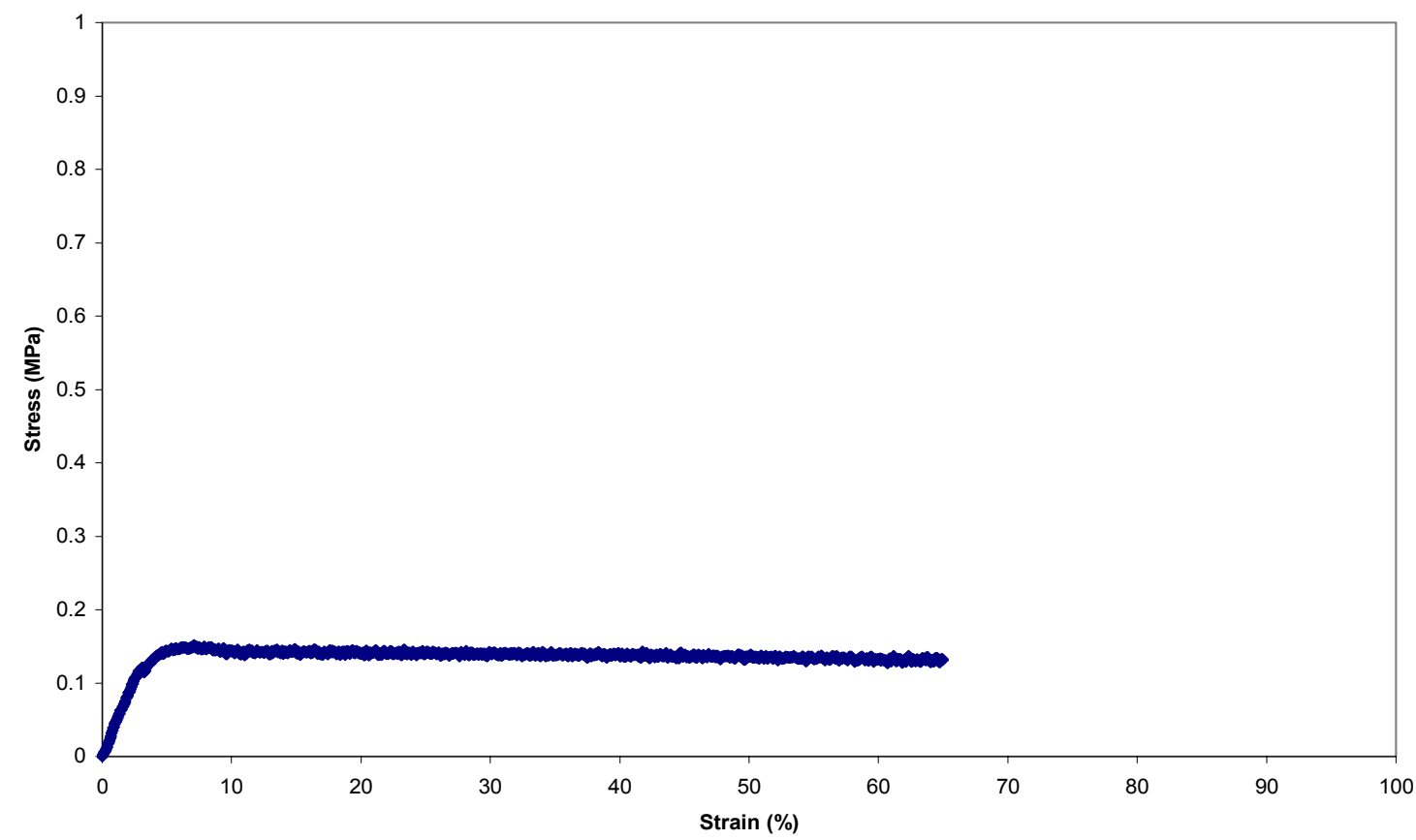

2.1.2

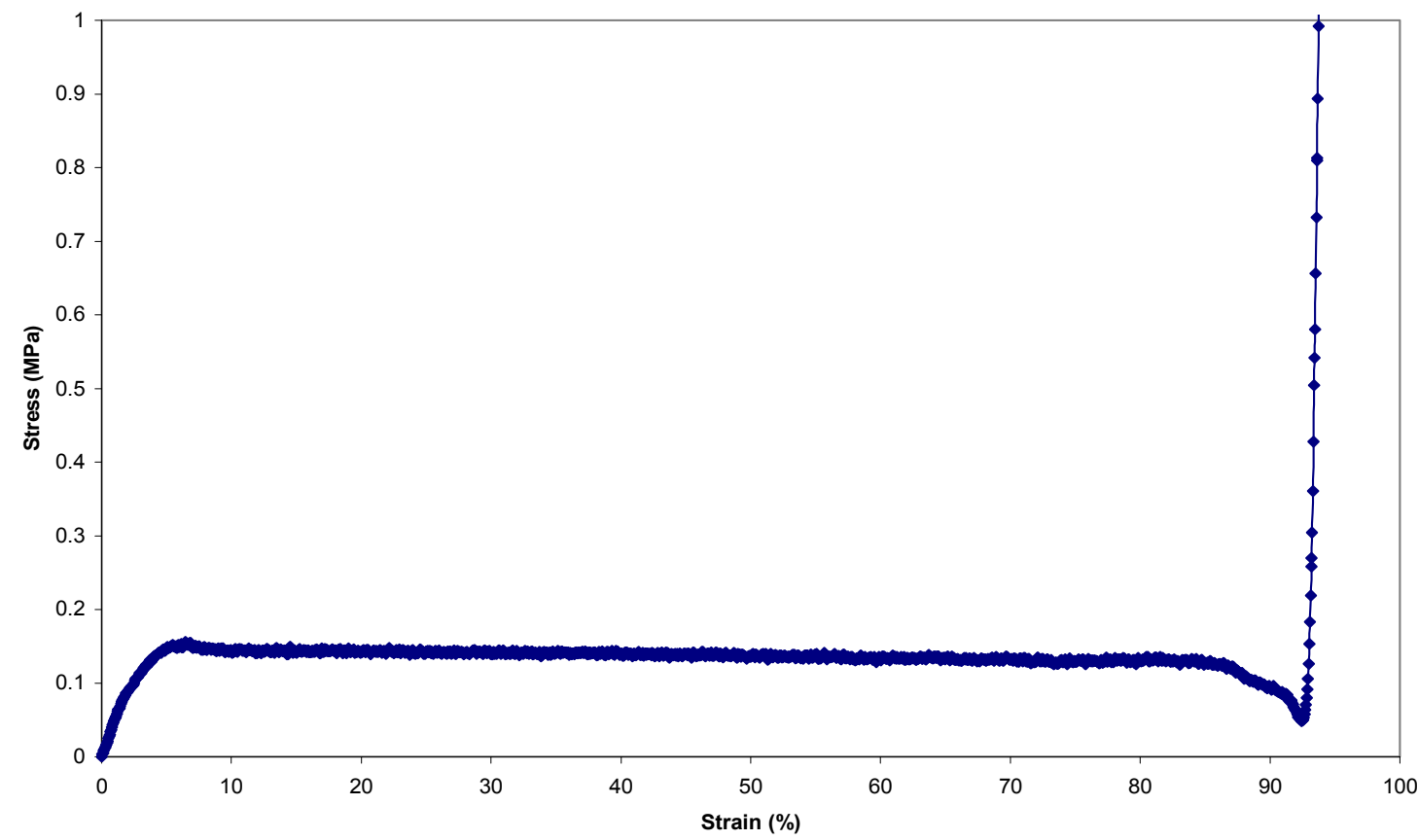

Figure A.1. Stress-Strain Plots for Samples 1 and 2 of Test 2.1. 
2.1.3

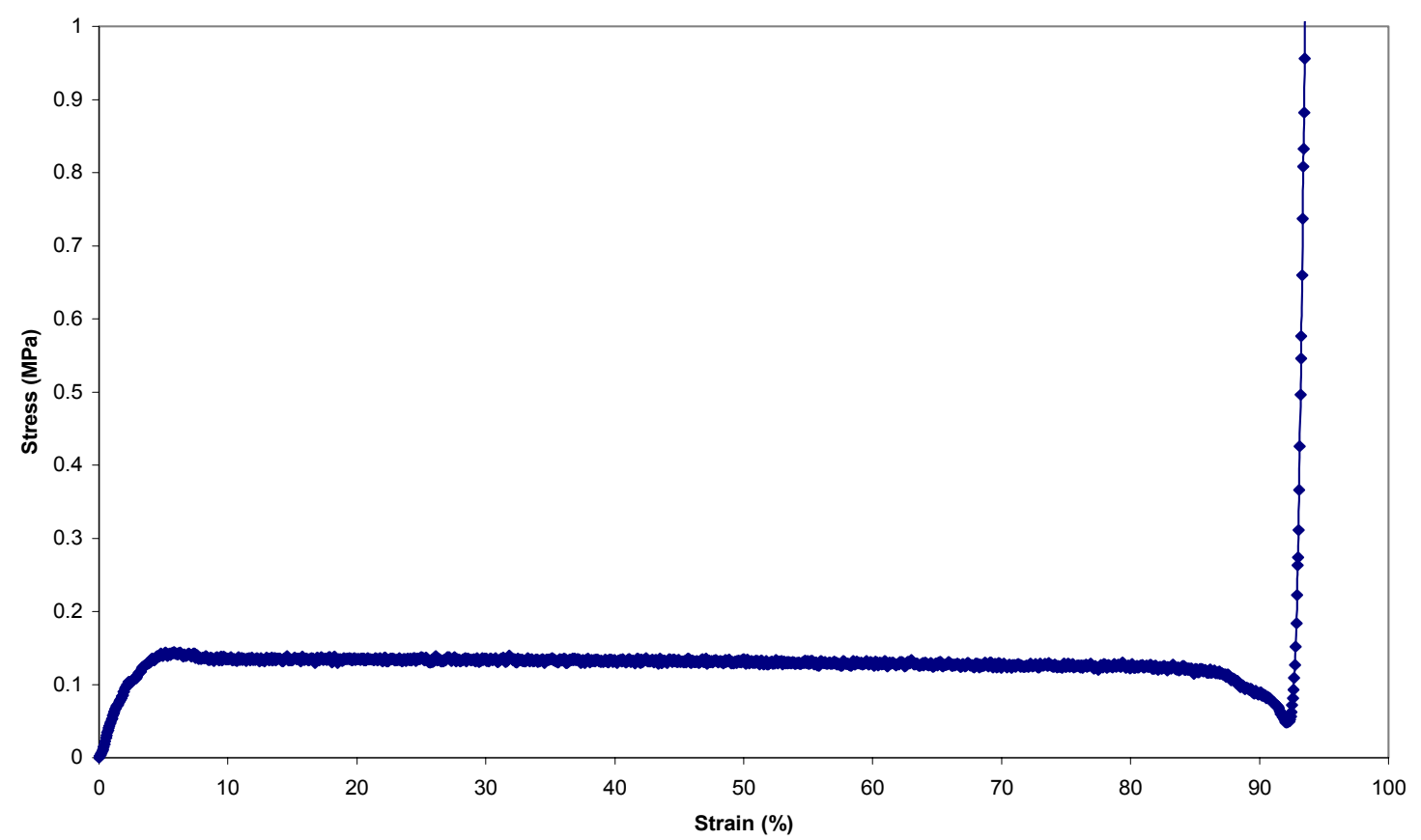

2.1.4

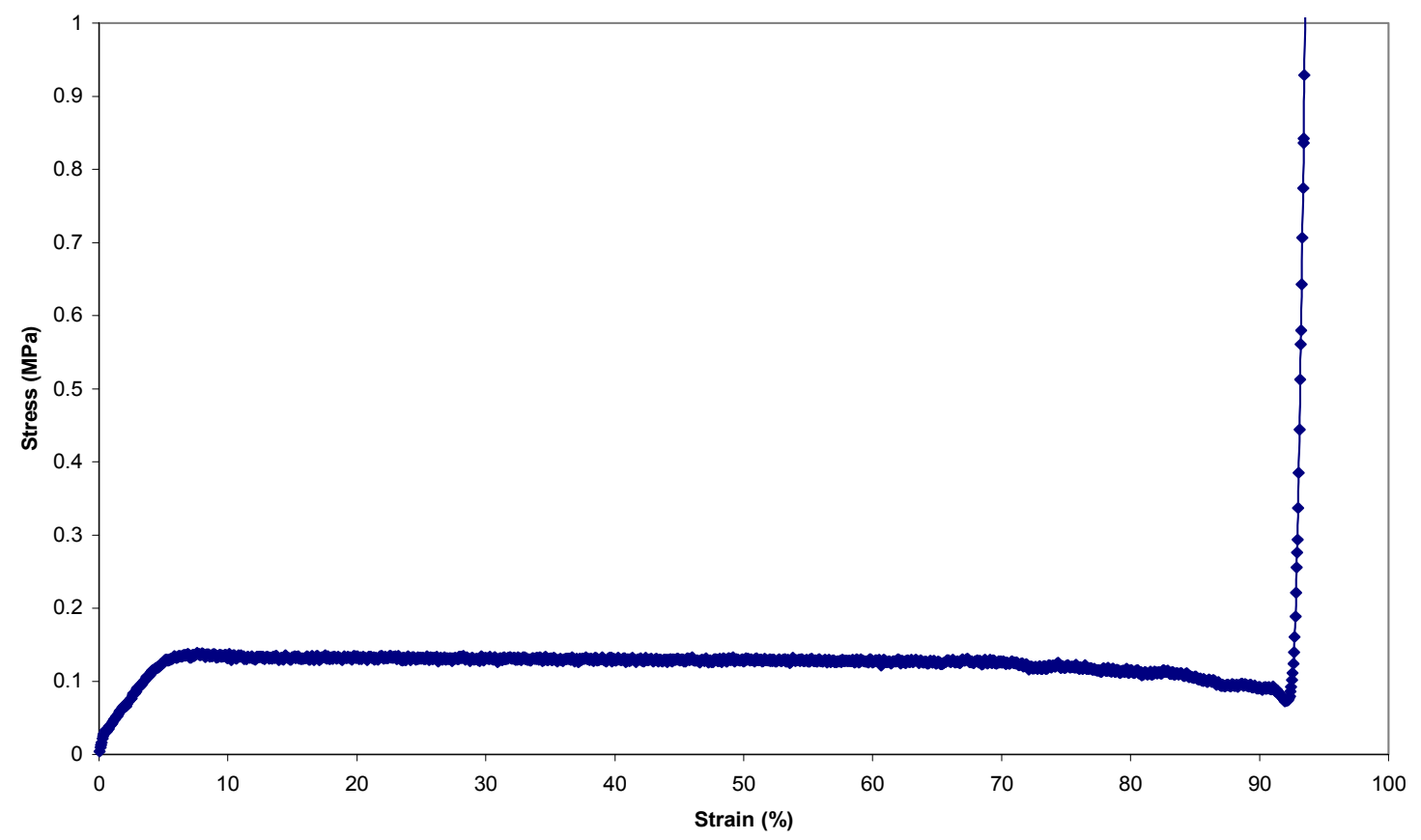

Figure A.2. Stress-Strain Plots for Samples 3 and 4 of Test 2.1. 


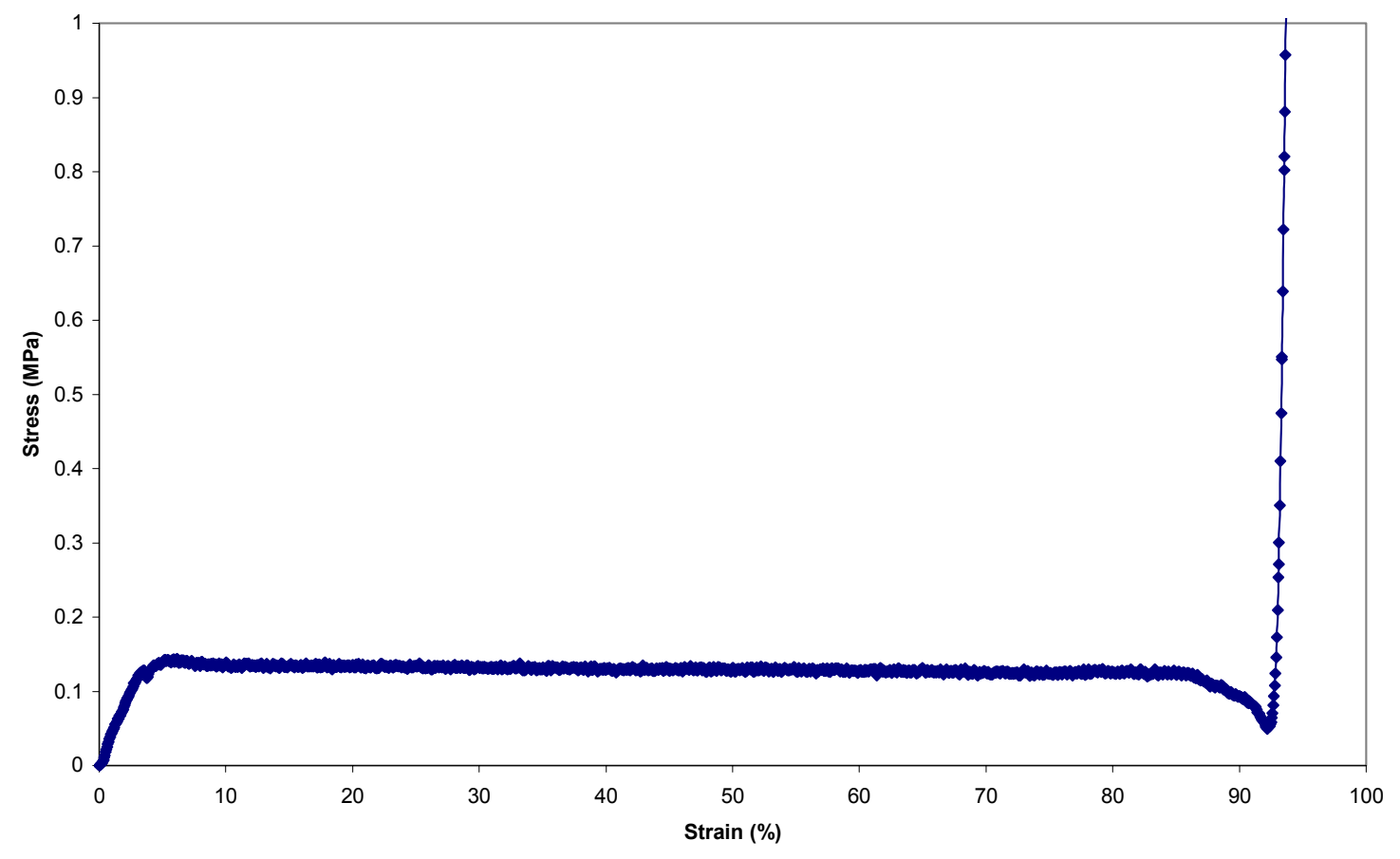

2.1.6

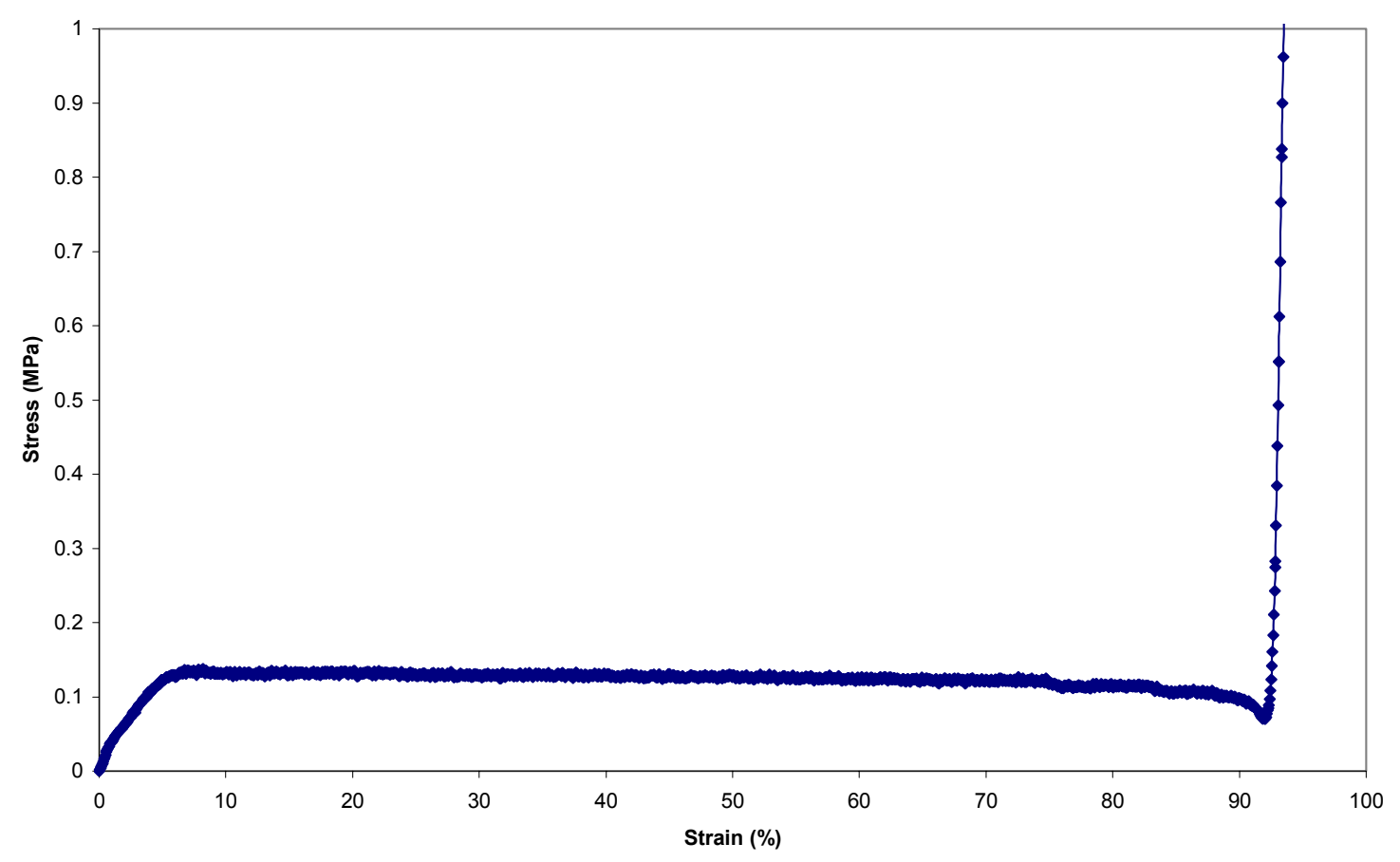

Figure A.3. Stress-Strain Plots for Samples 5 and 6 of Test 2.1. 
2.1.7

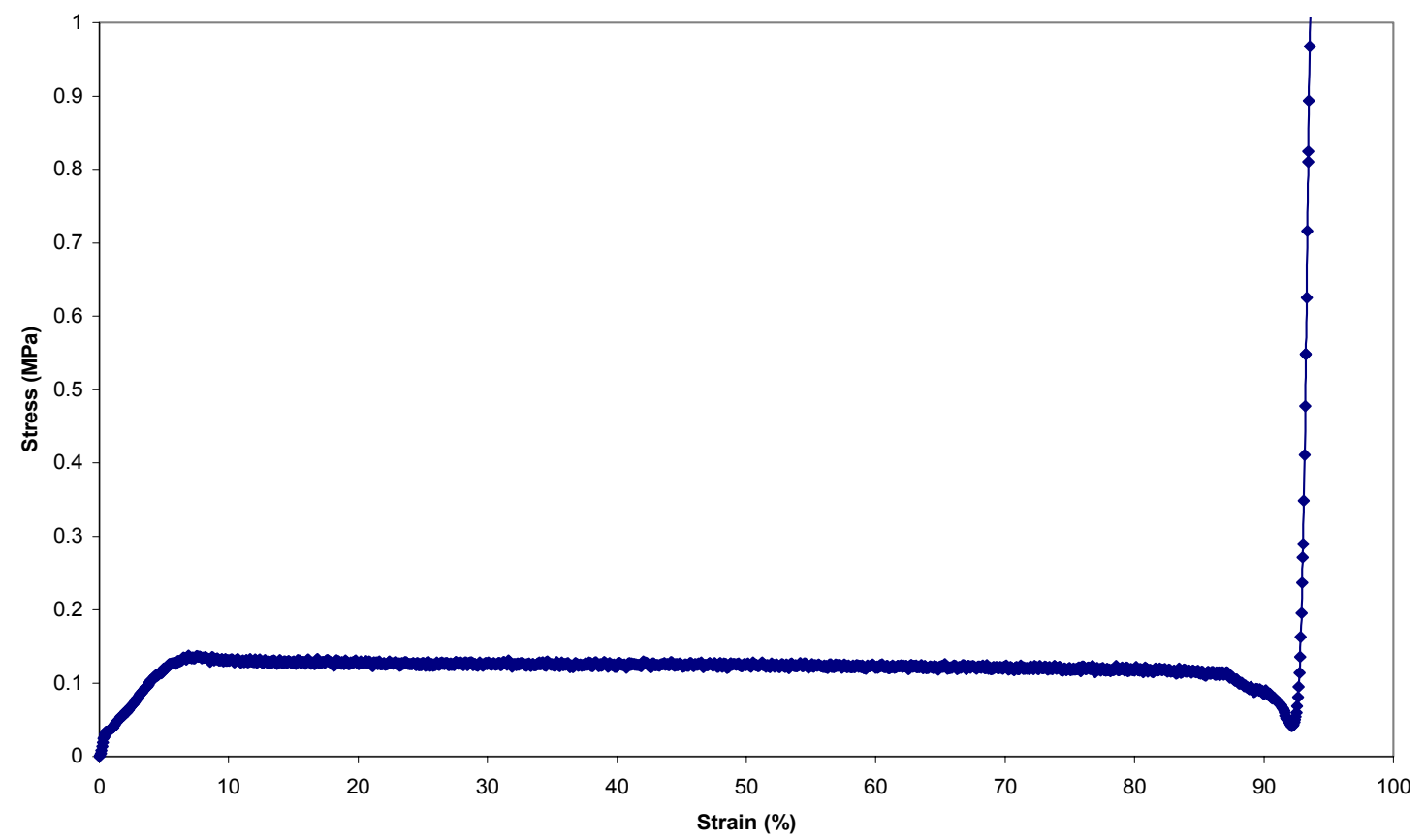

2.1.8

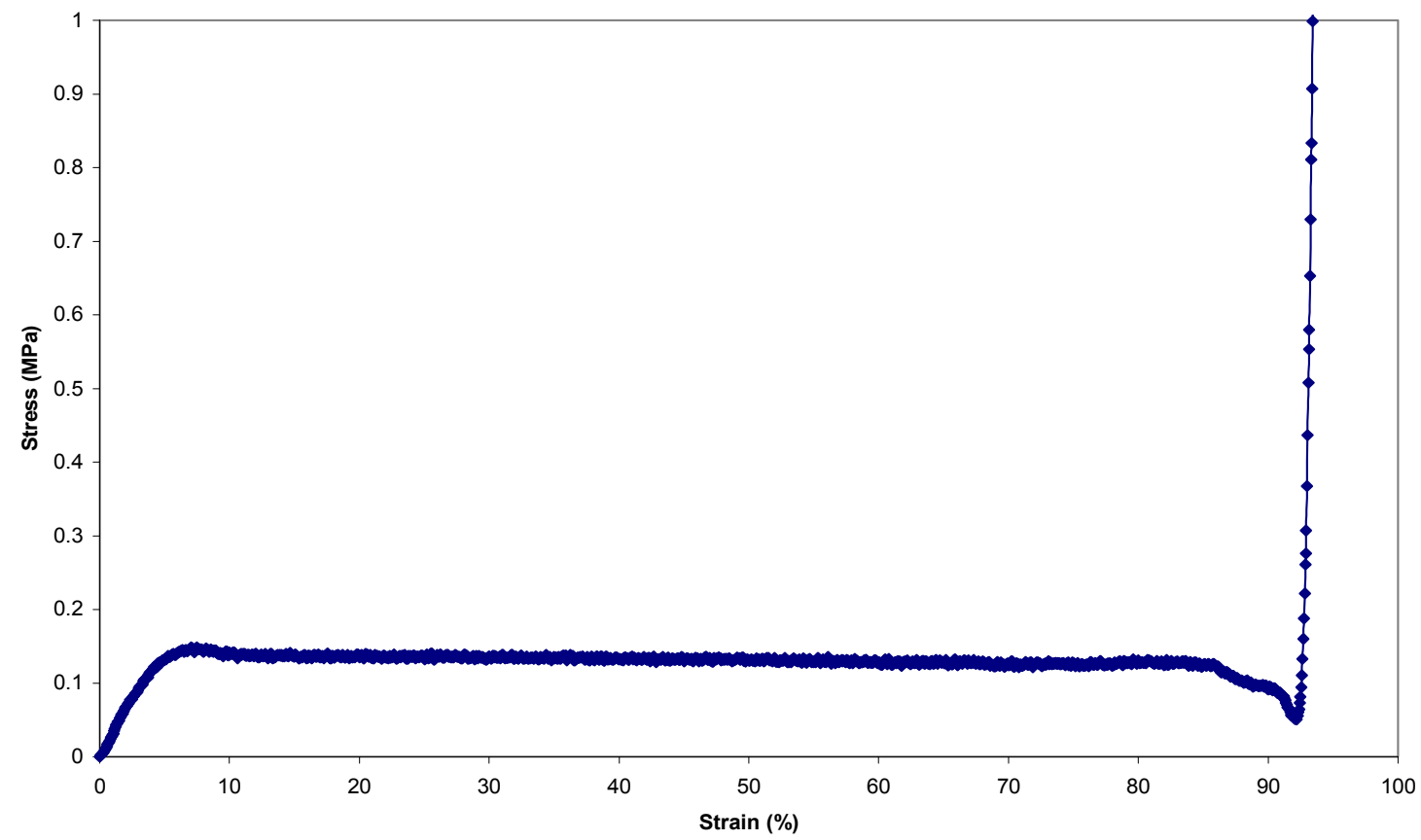

Figure A.4. Stress-Strain Plots for Samples 7 and 8 of Test 2.1. 
2.1.9

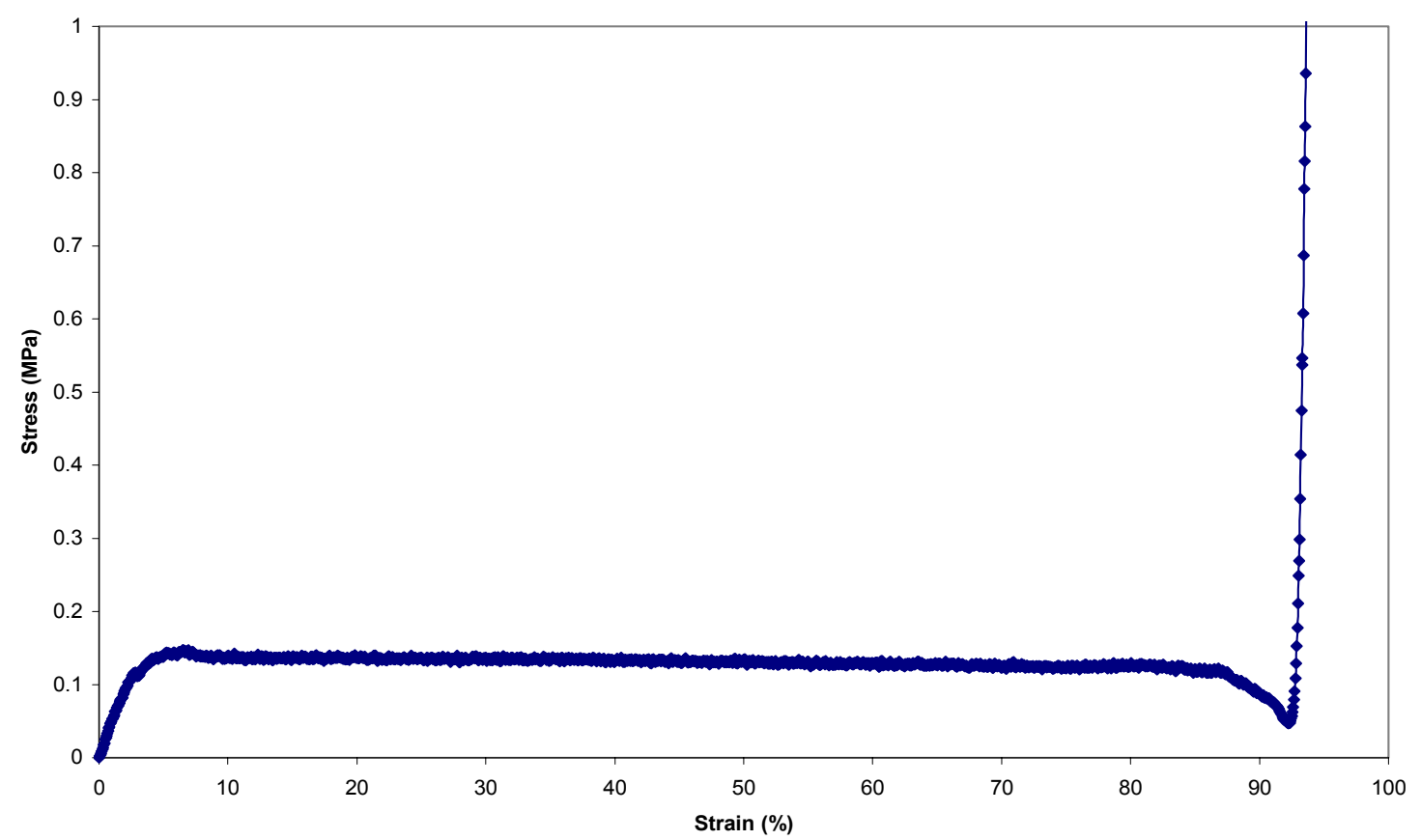

2.1.10

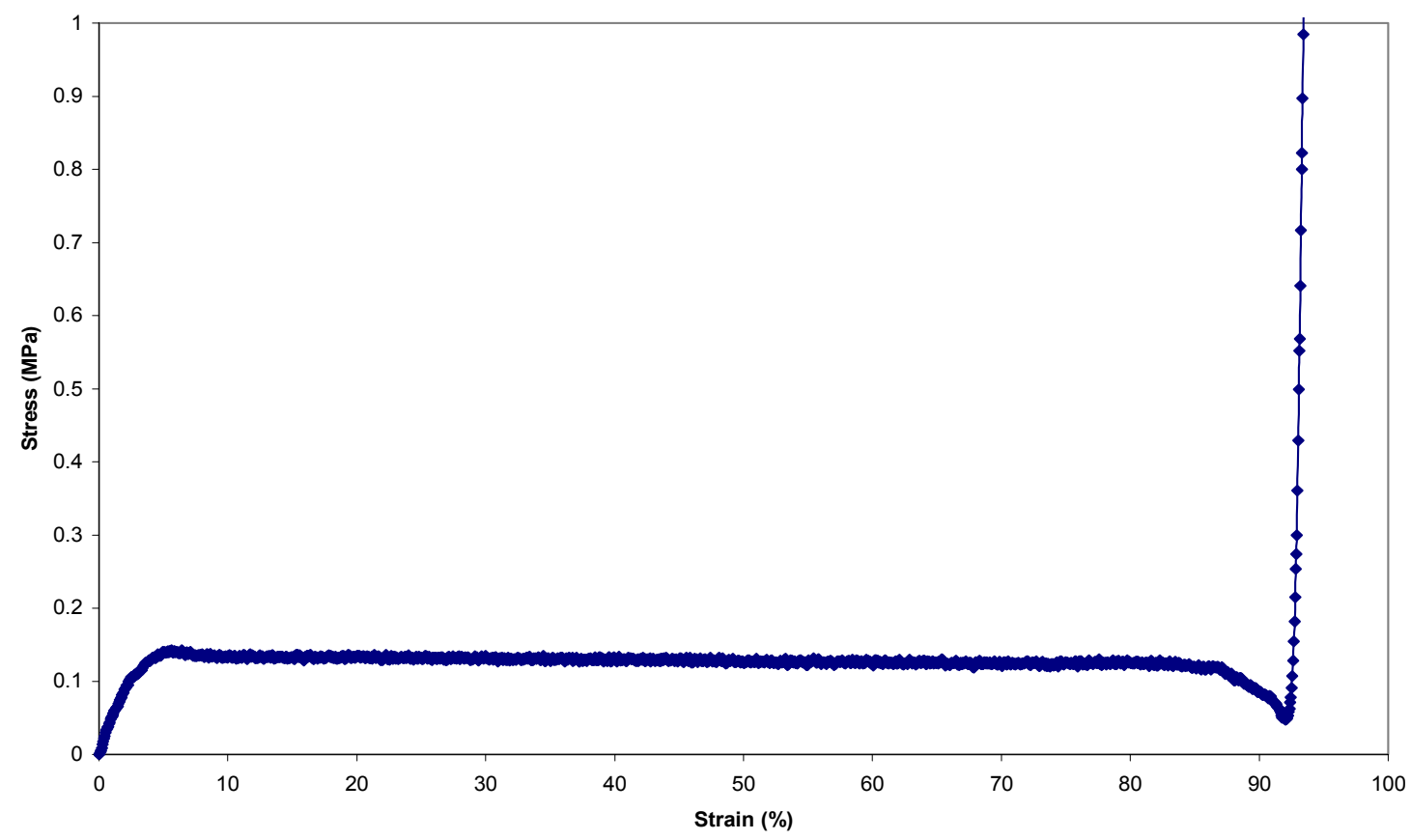

Figure A.5. Stress-Strain Plots for Samples 9 and 10 of Test 2.1. 


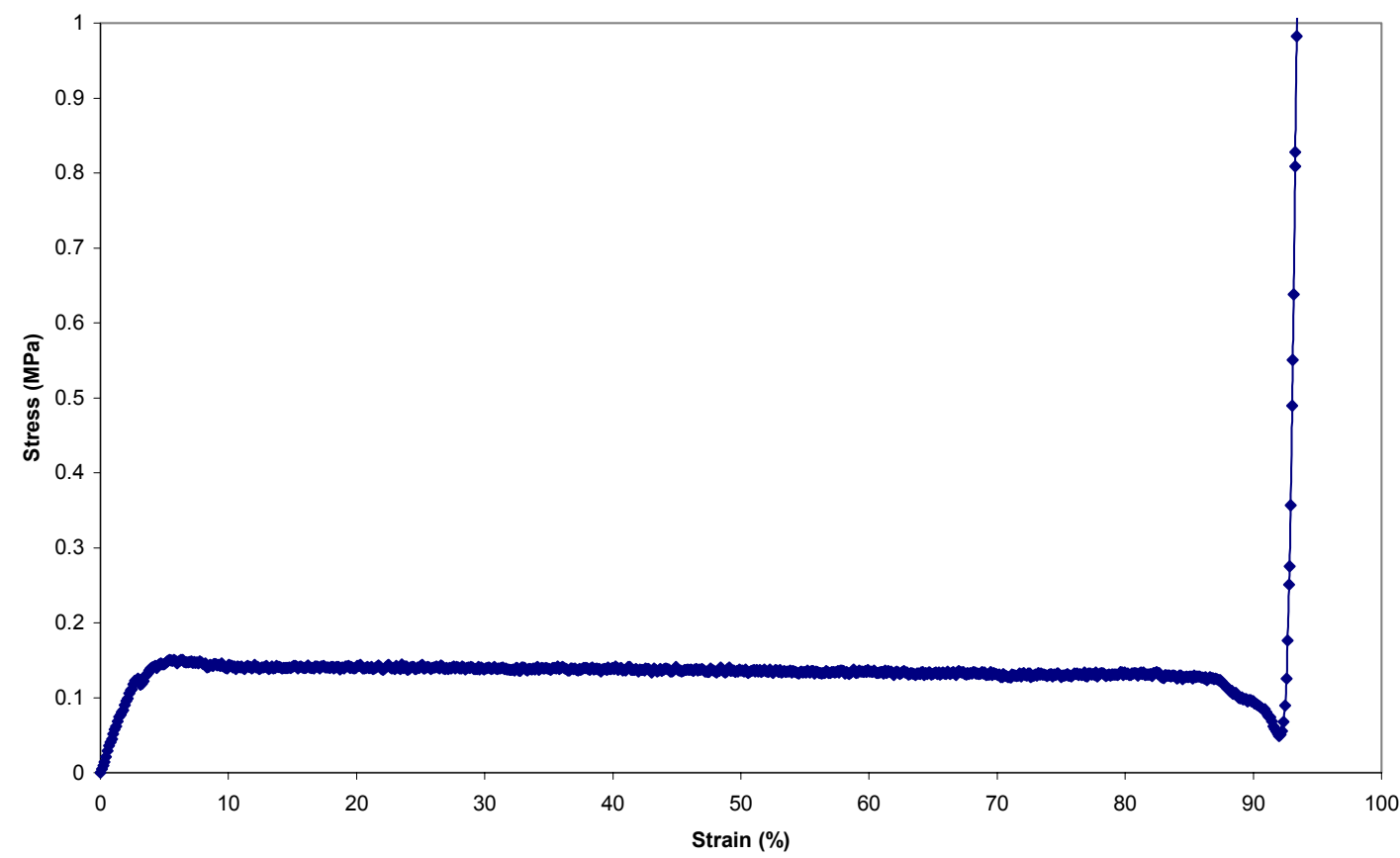

2.2.2

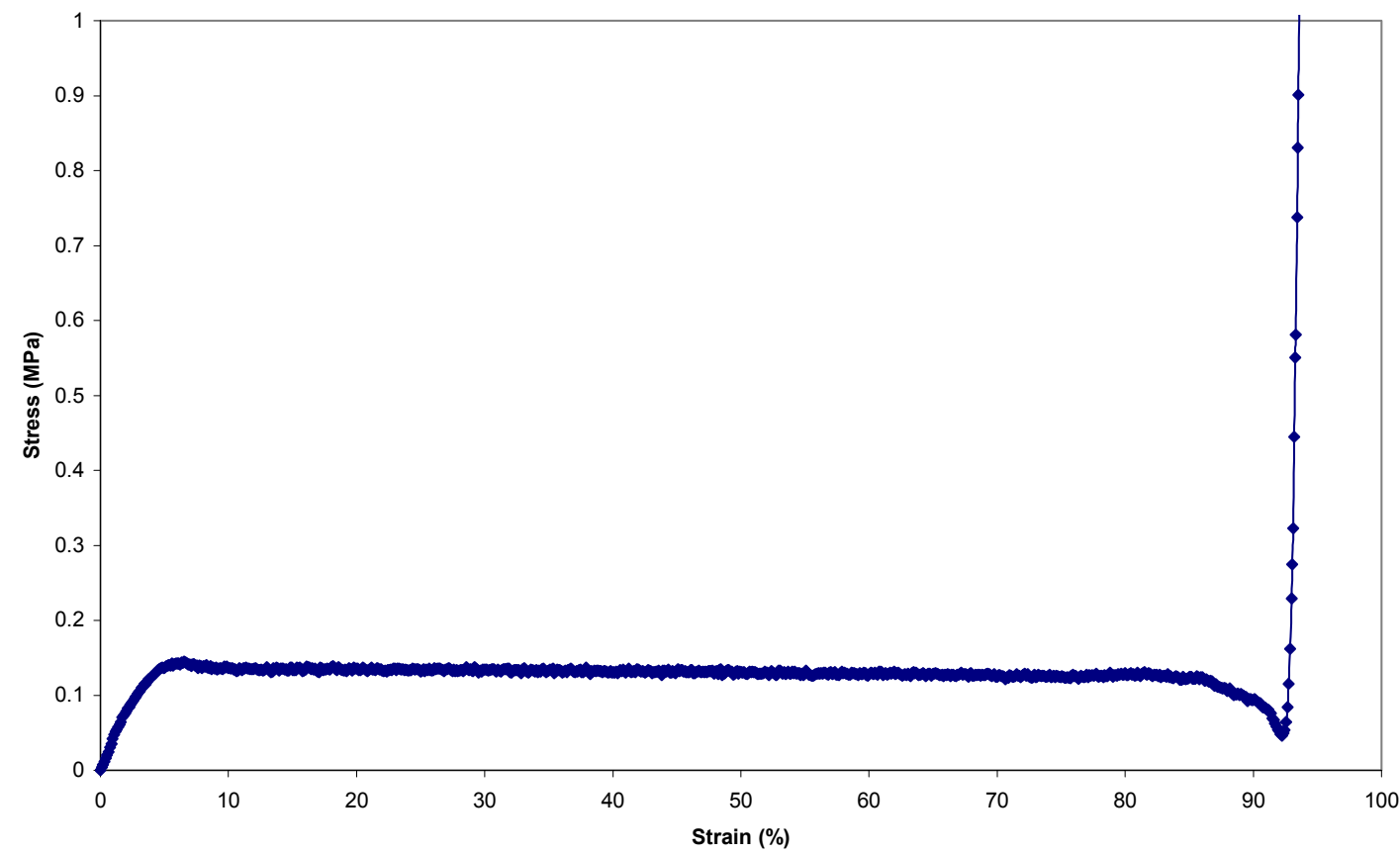

Figure A.6. Stress-Strain Plots for Samples 1 and 2 of Test 2.2. 


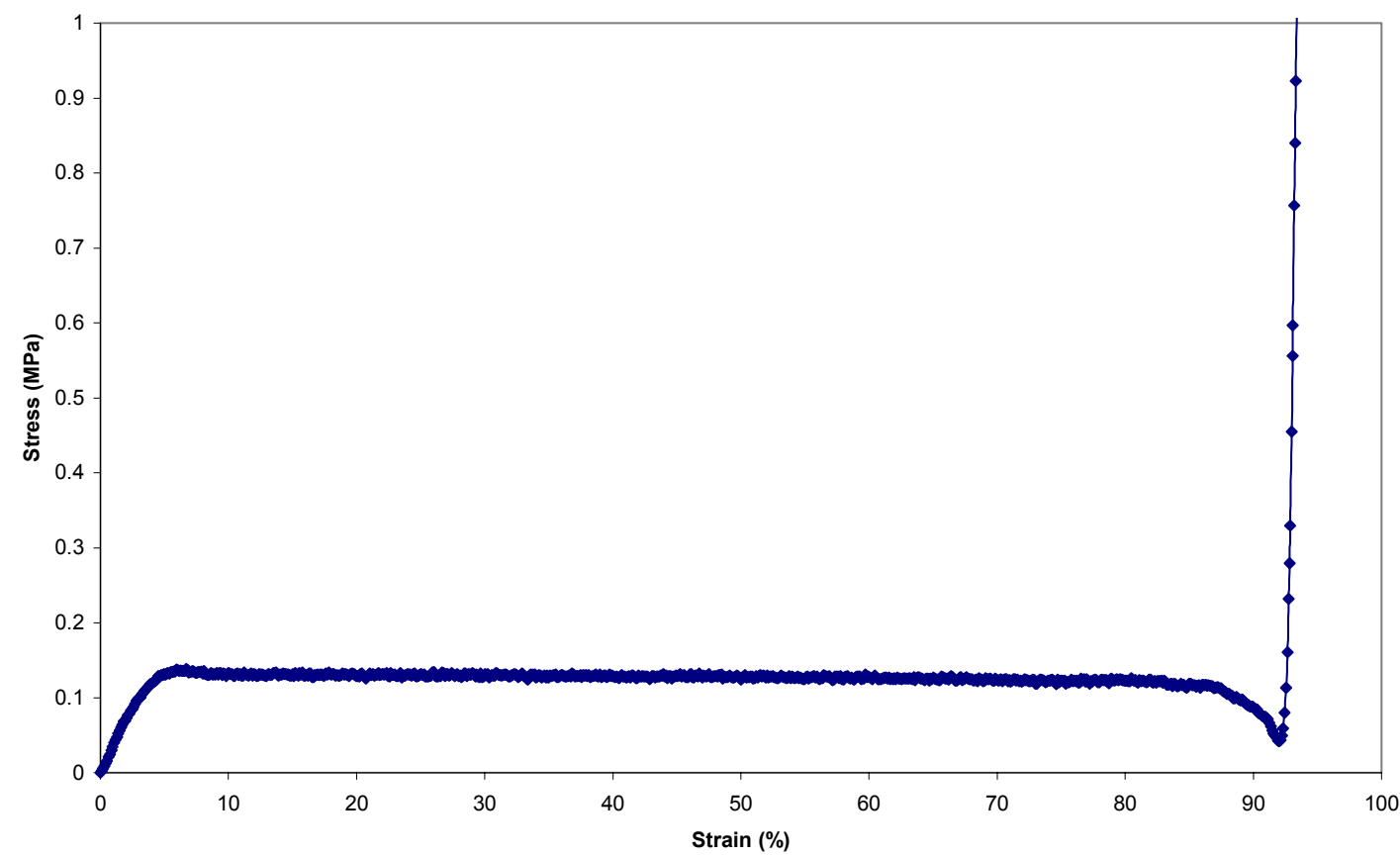

2.2.4

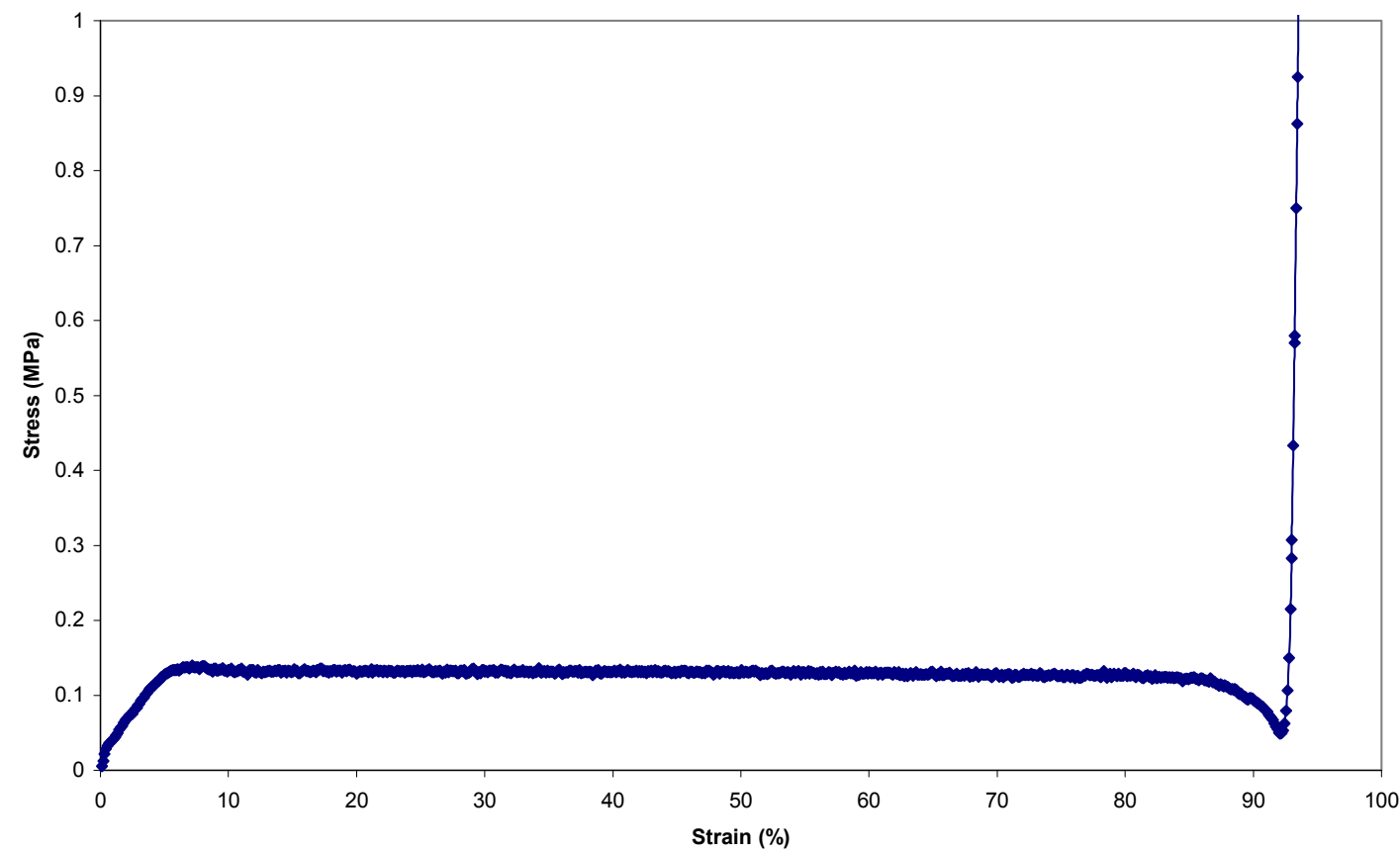

Figure A.7. Stress-Strain Plots for Samples 3 and 4 of Test 2.2. 
2.2.5

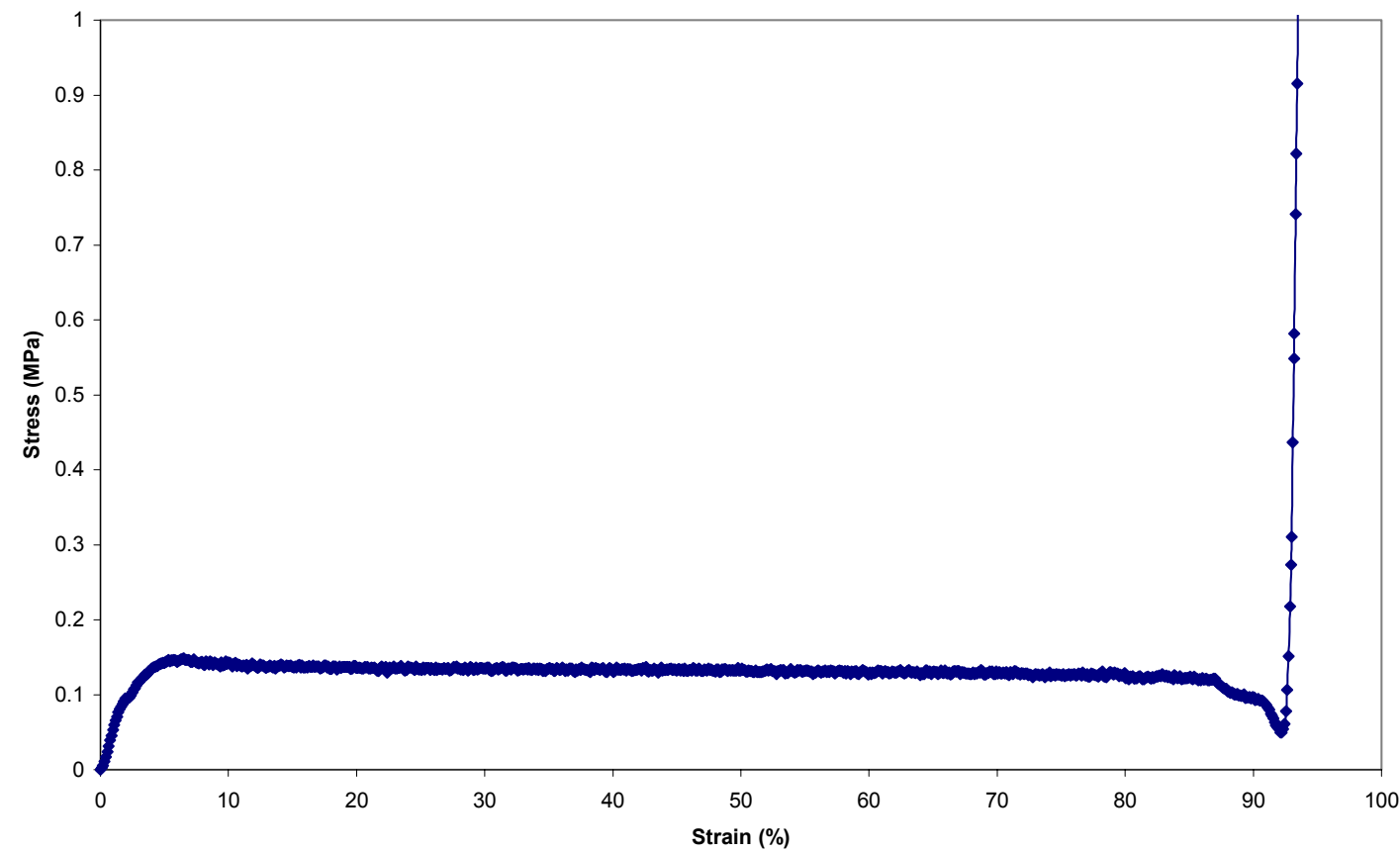

2.2.6

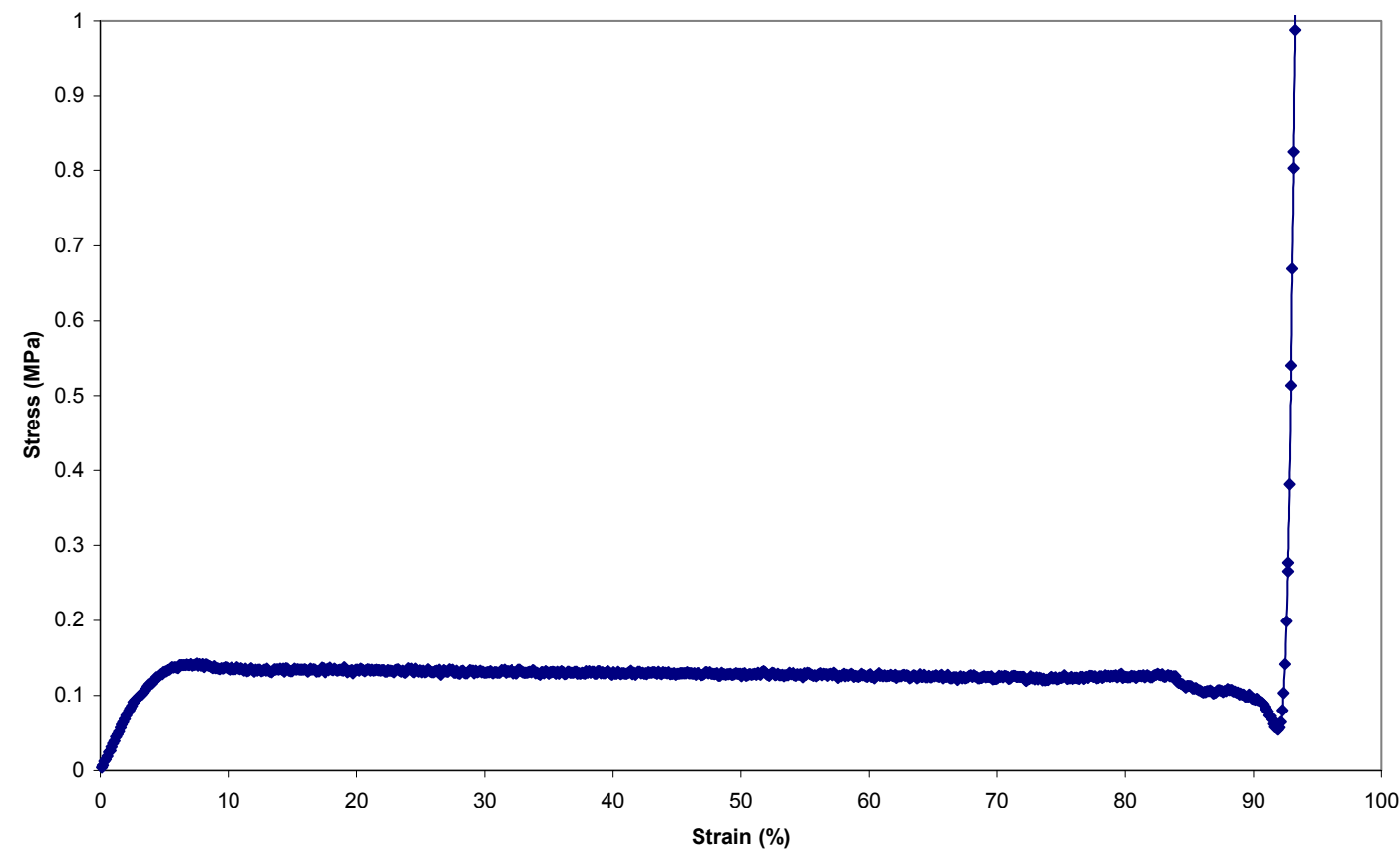

Figure A.8. Stress-Strain Plots for Samples 5 and 6 of Test 2.2. 
2.2.7

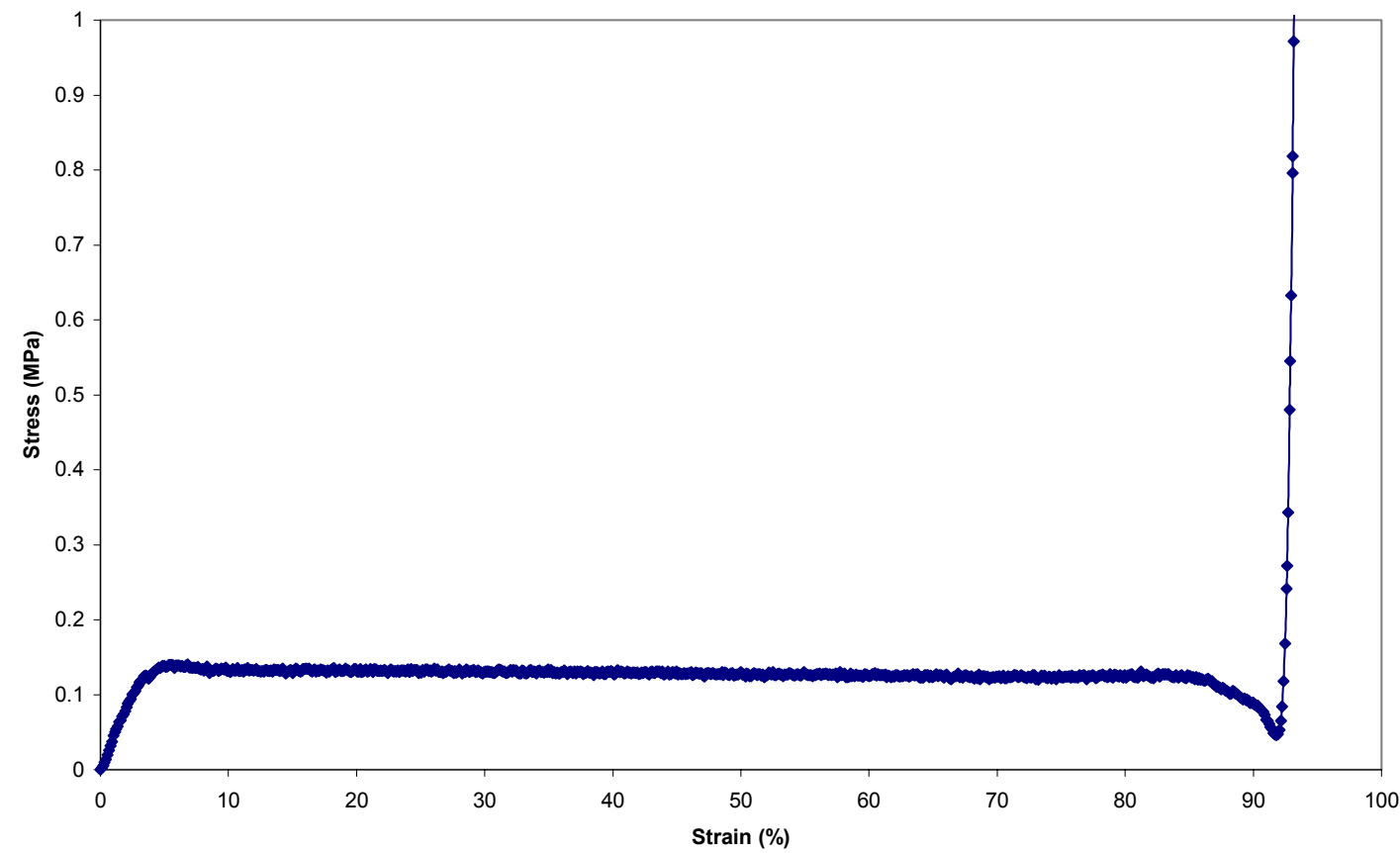

2.2.8

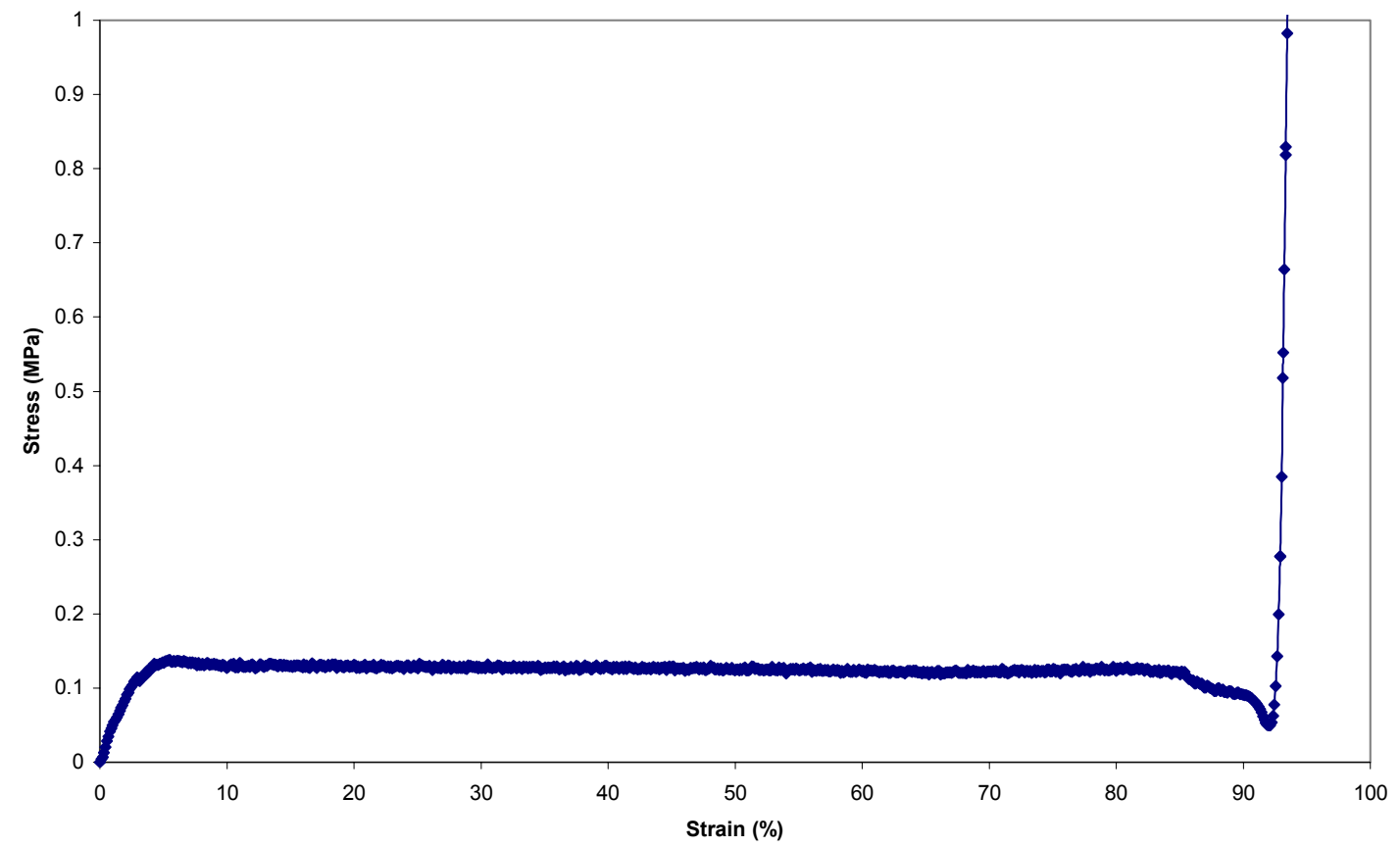

Figure A.9. Stress-Strain Plots for Samples 7 and 8 of Test 2.2. 
2.2.9

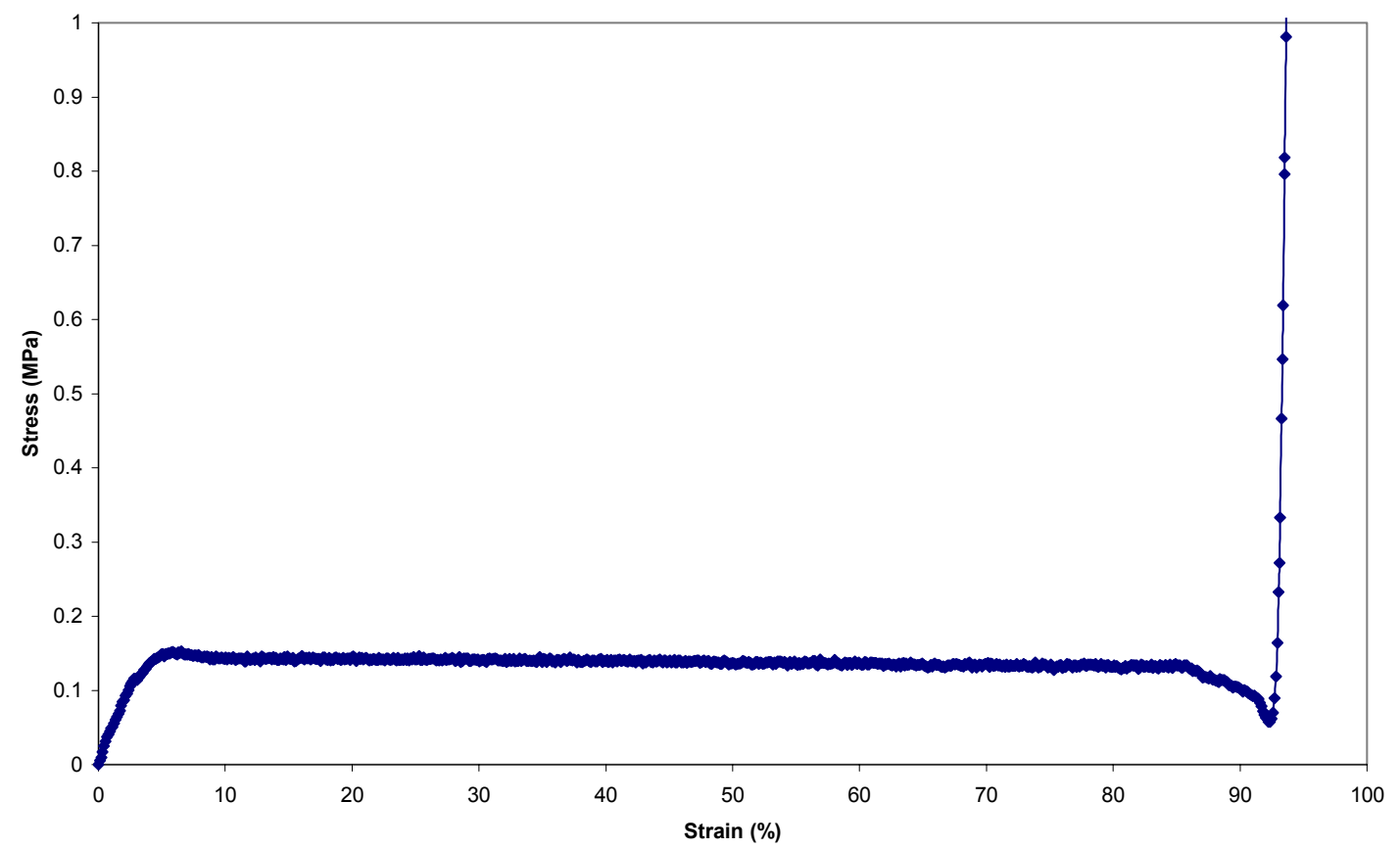

2.2.10

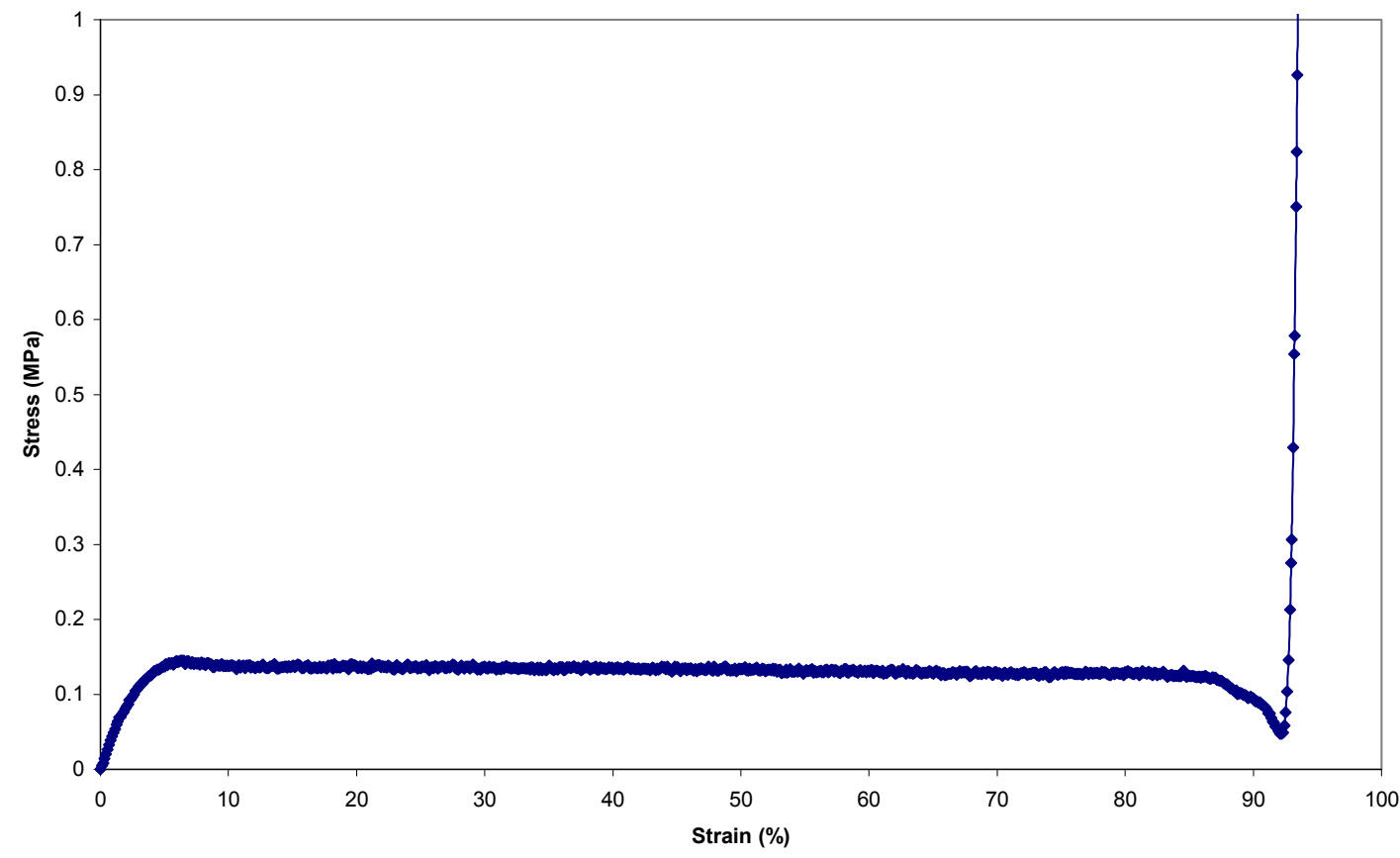

Figure A.10. Stress-Strain Plots for Samples 9 and 10 of Test 2.2. 


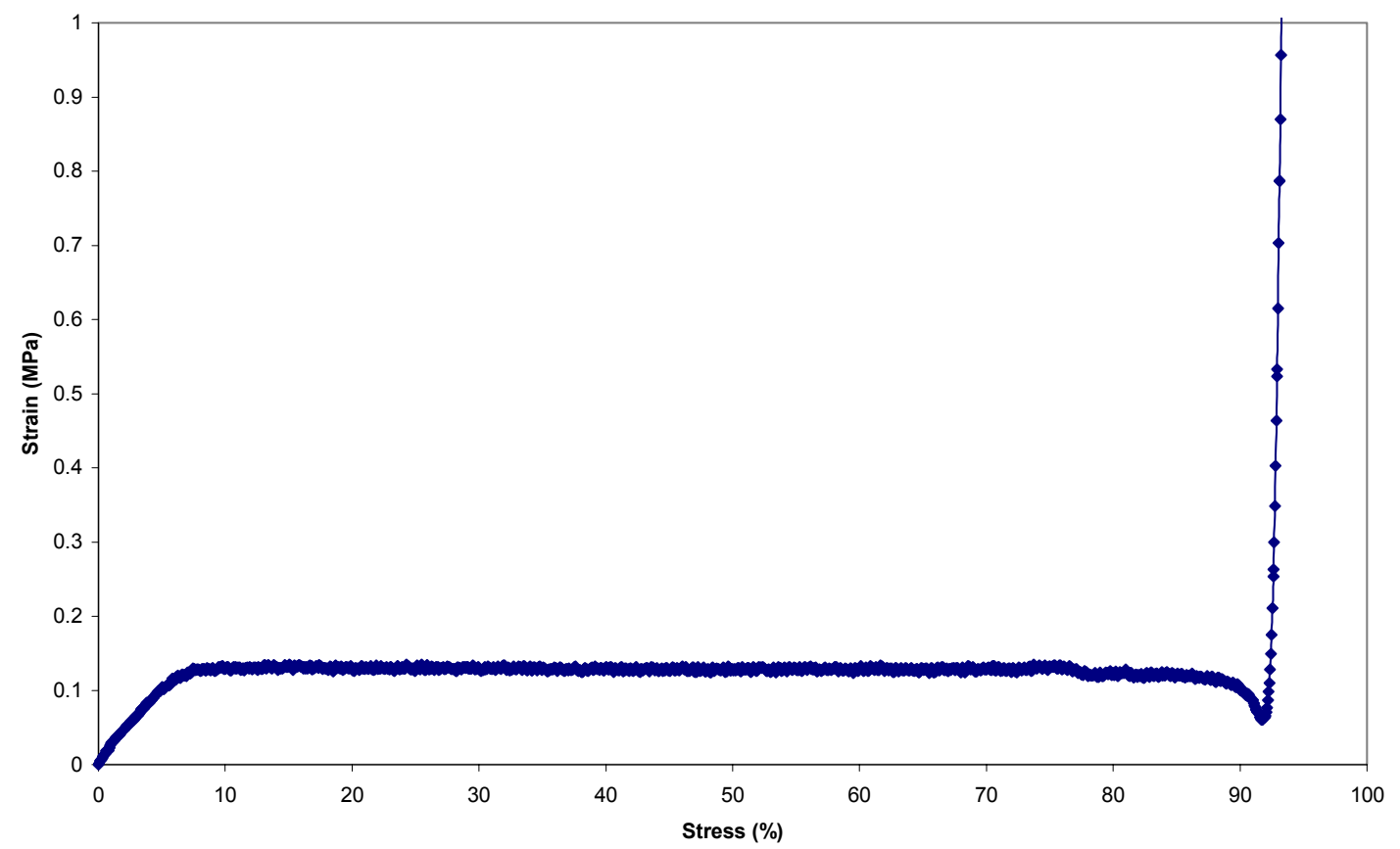

2.3.2

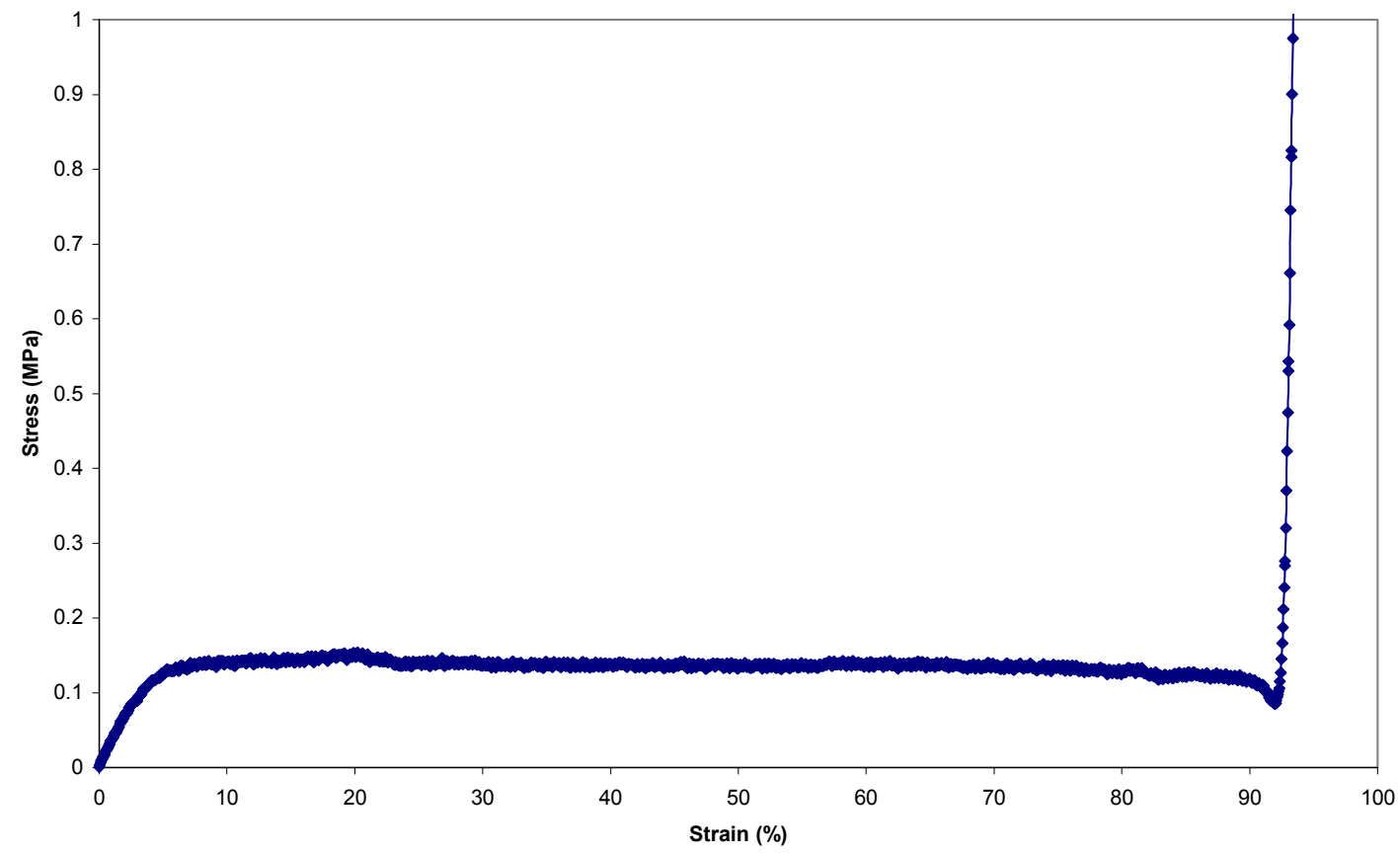

Figure A.11. Stress-Strain Plots for Samples 1 and 2 of Test 2.3. 
2.3.3

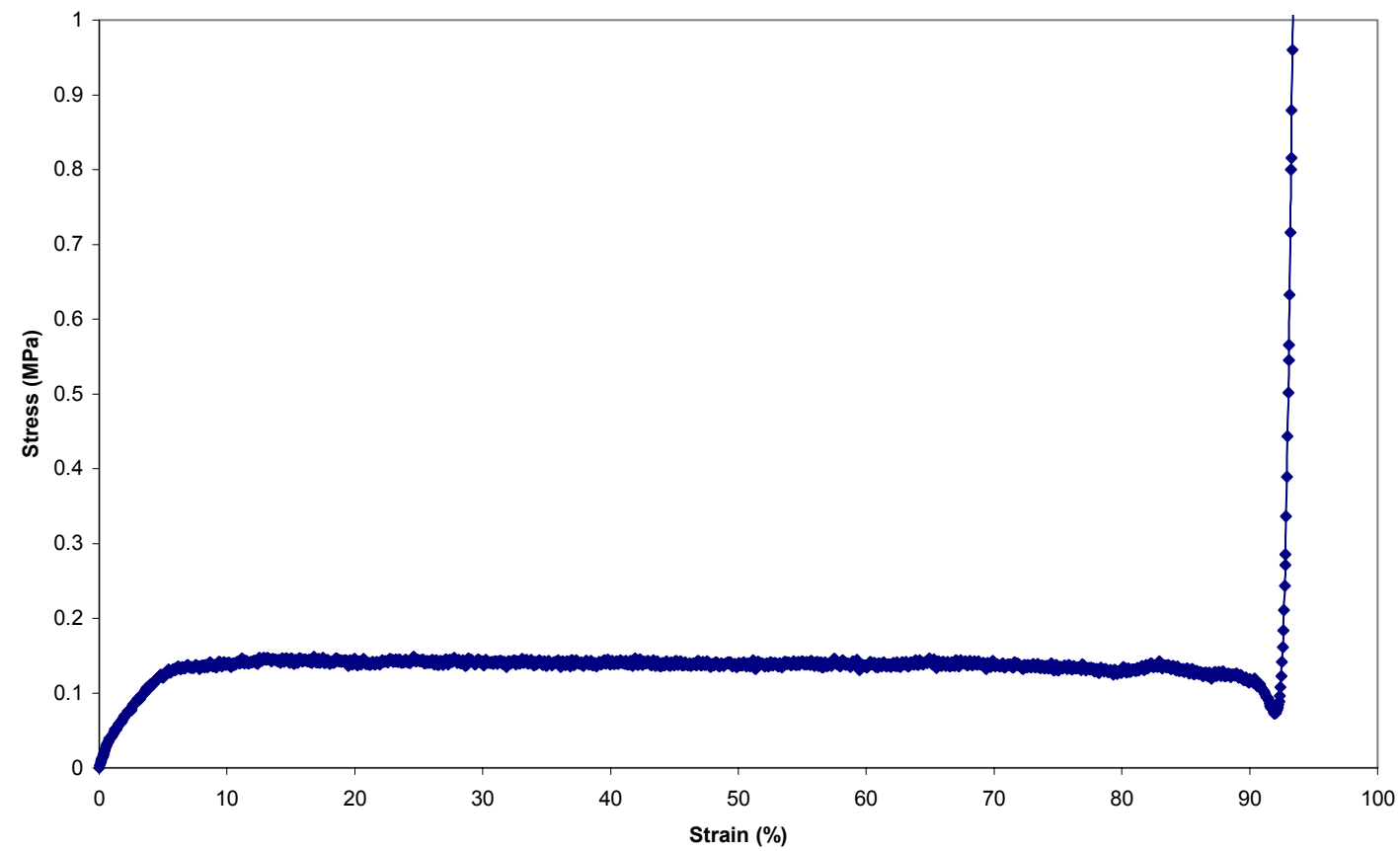

2.3.4

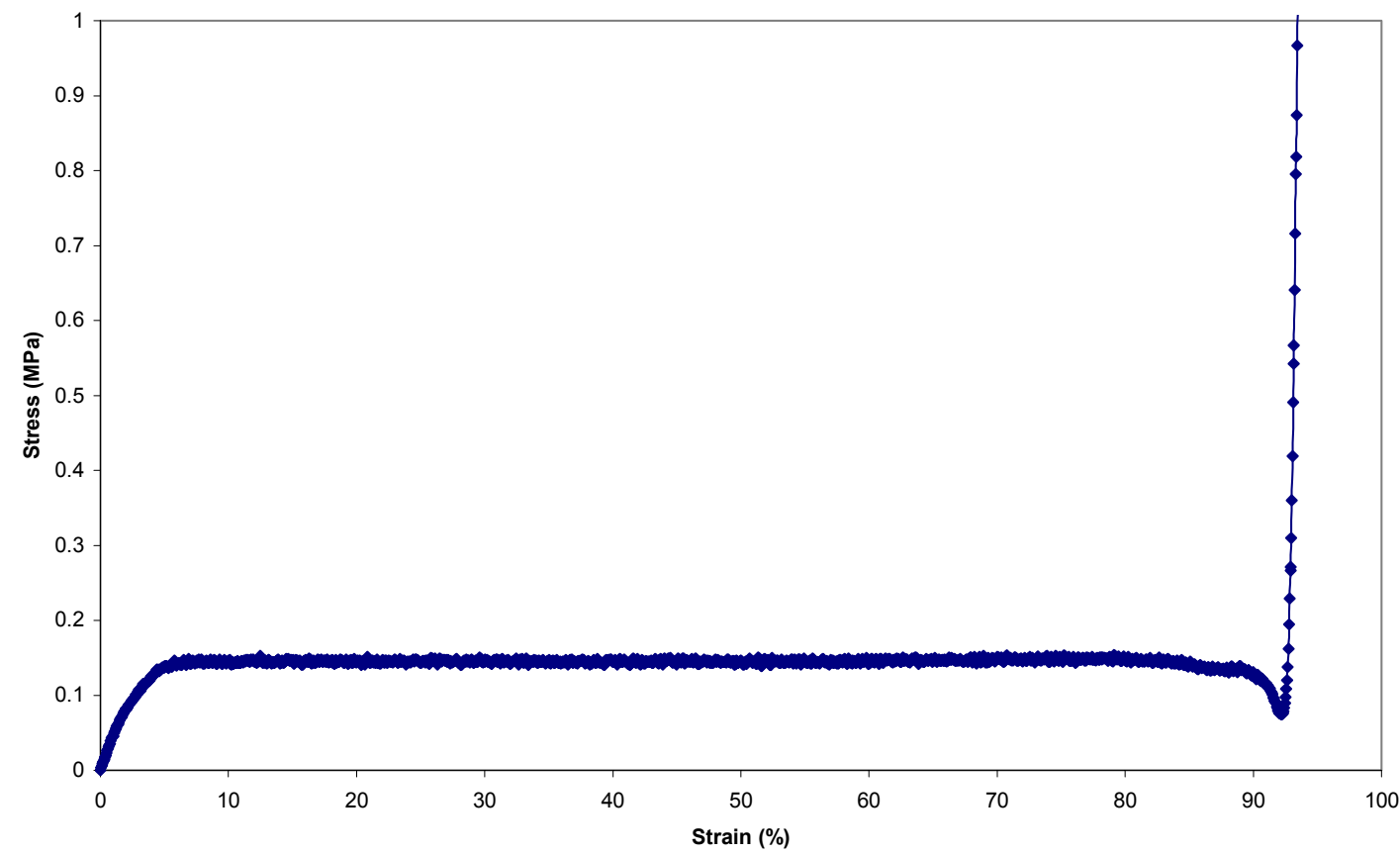

Figure A.12. Stress-Strain Plots for Samples 3 and 4 of Test 2.3. 
2.3.5

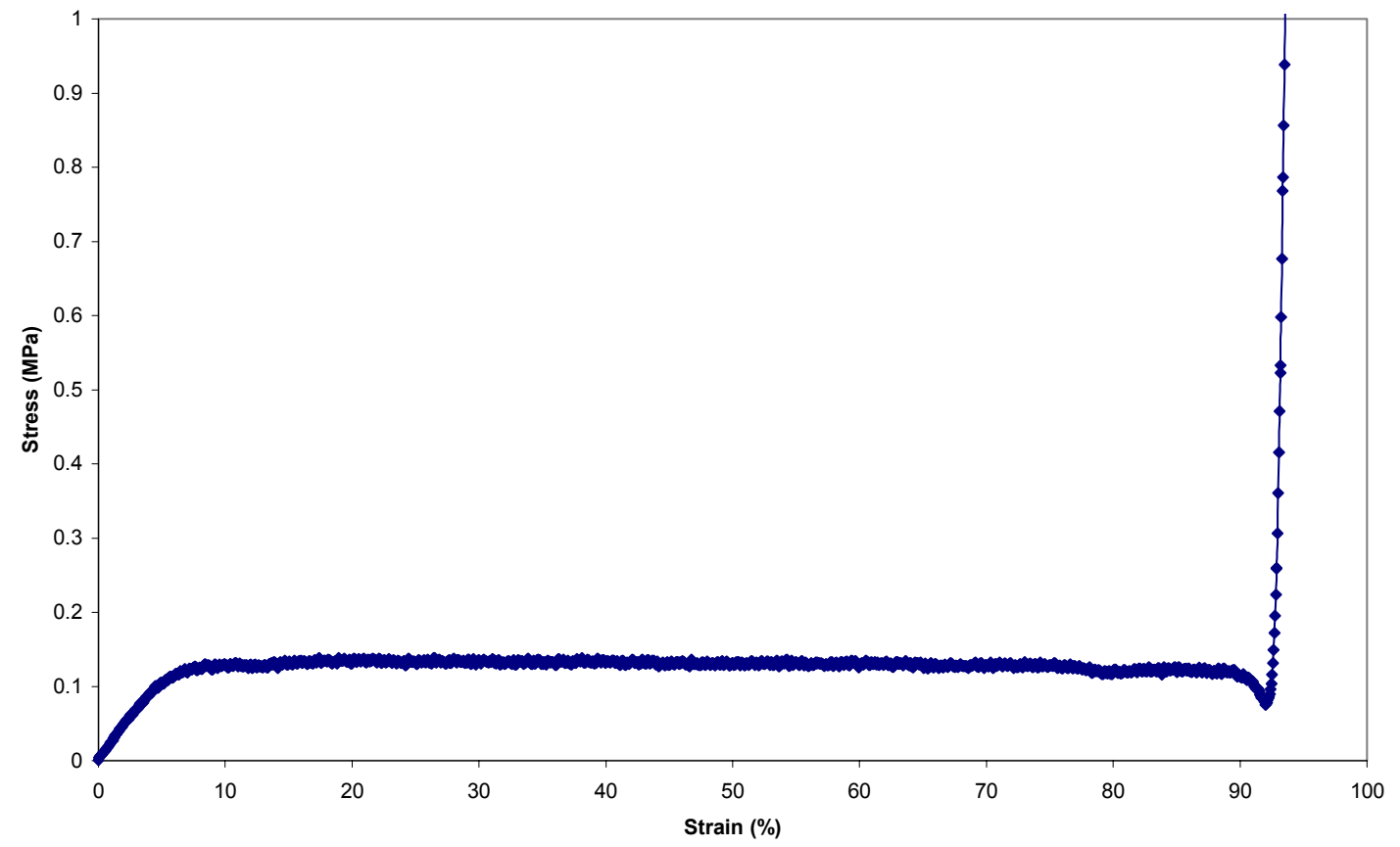

2.3.6

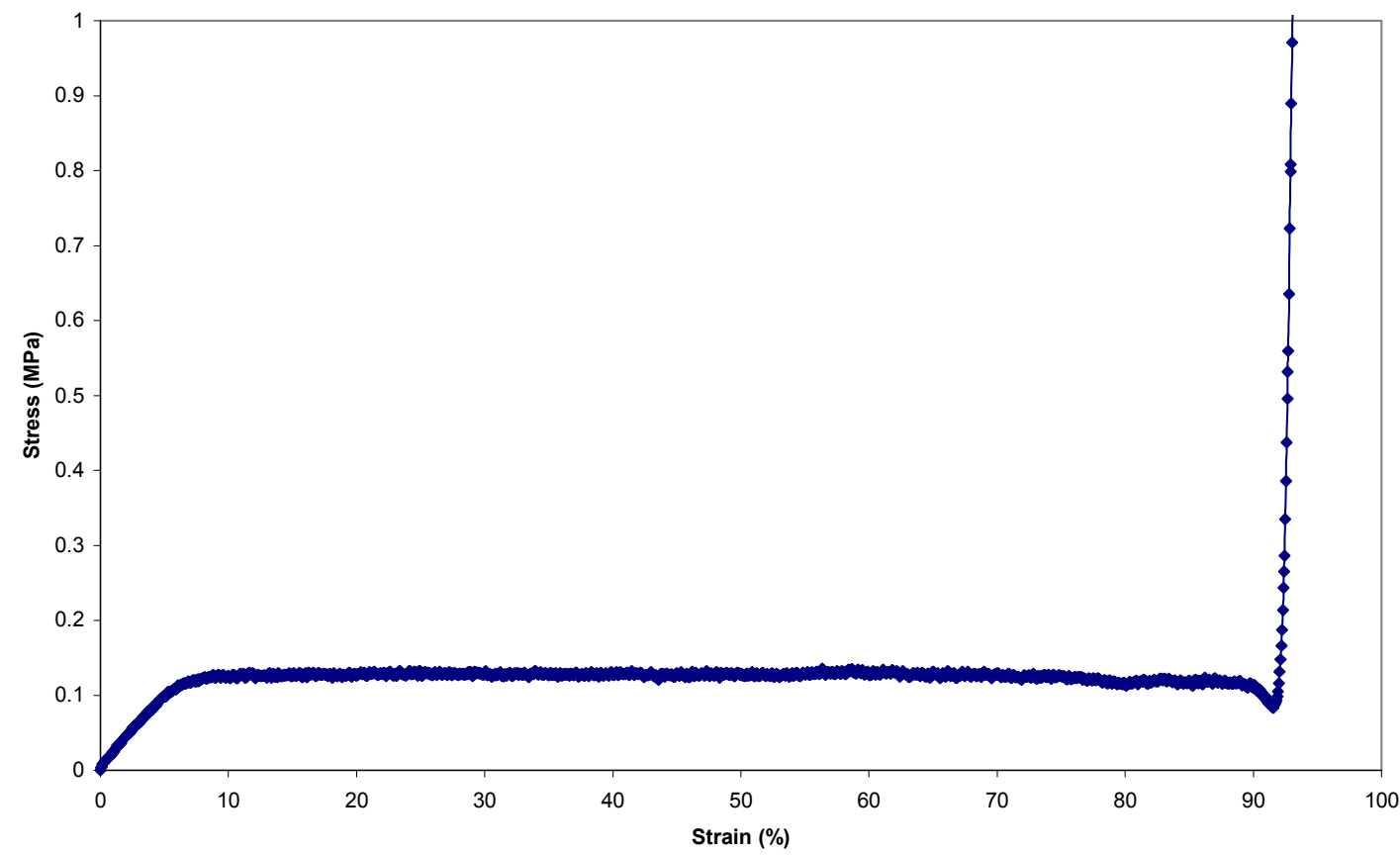

Figure A.13. Stress-Strain Plots for Samples 5 and 6 of Test 2.3. 
2.3.7

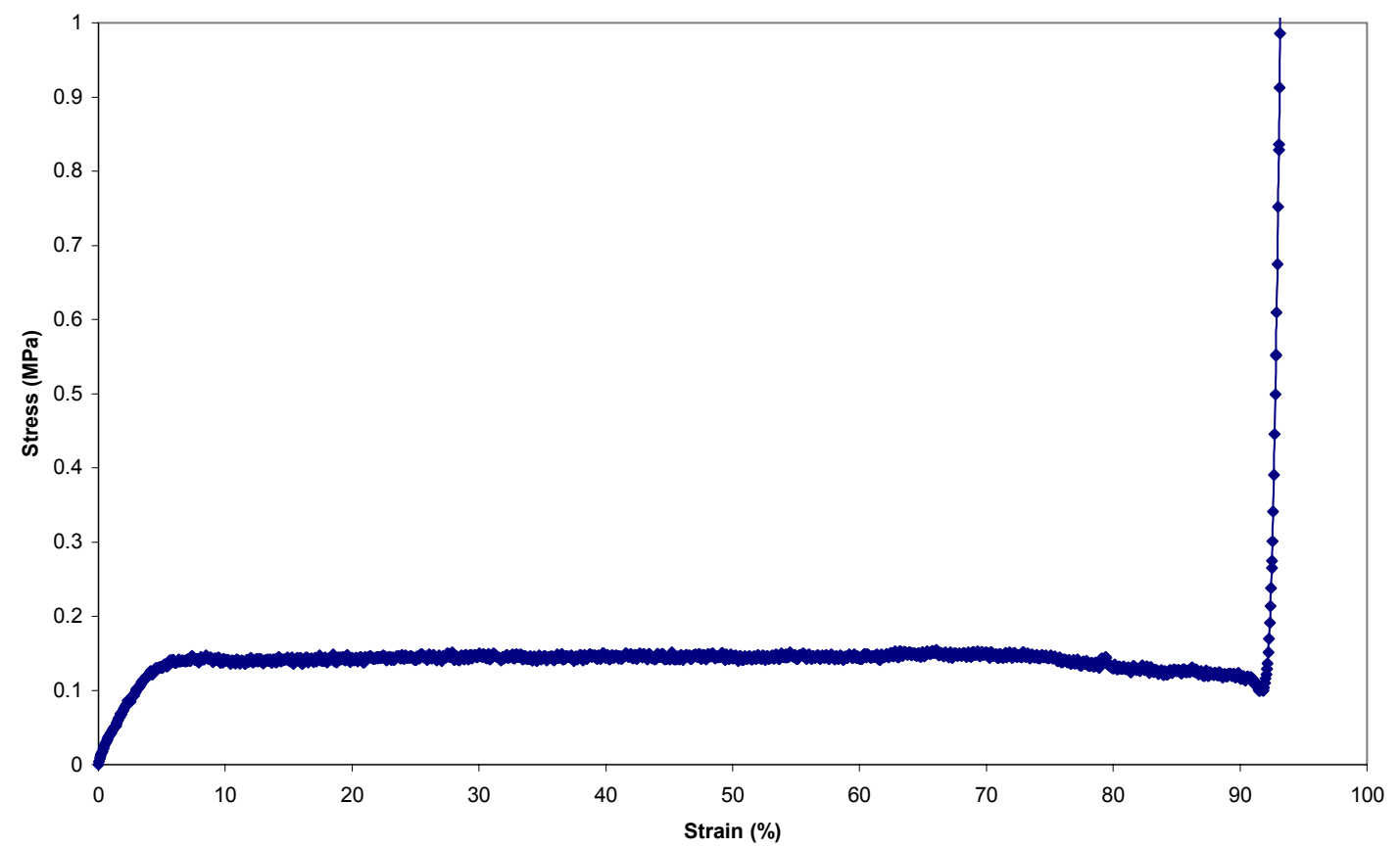

2.3.8

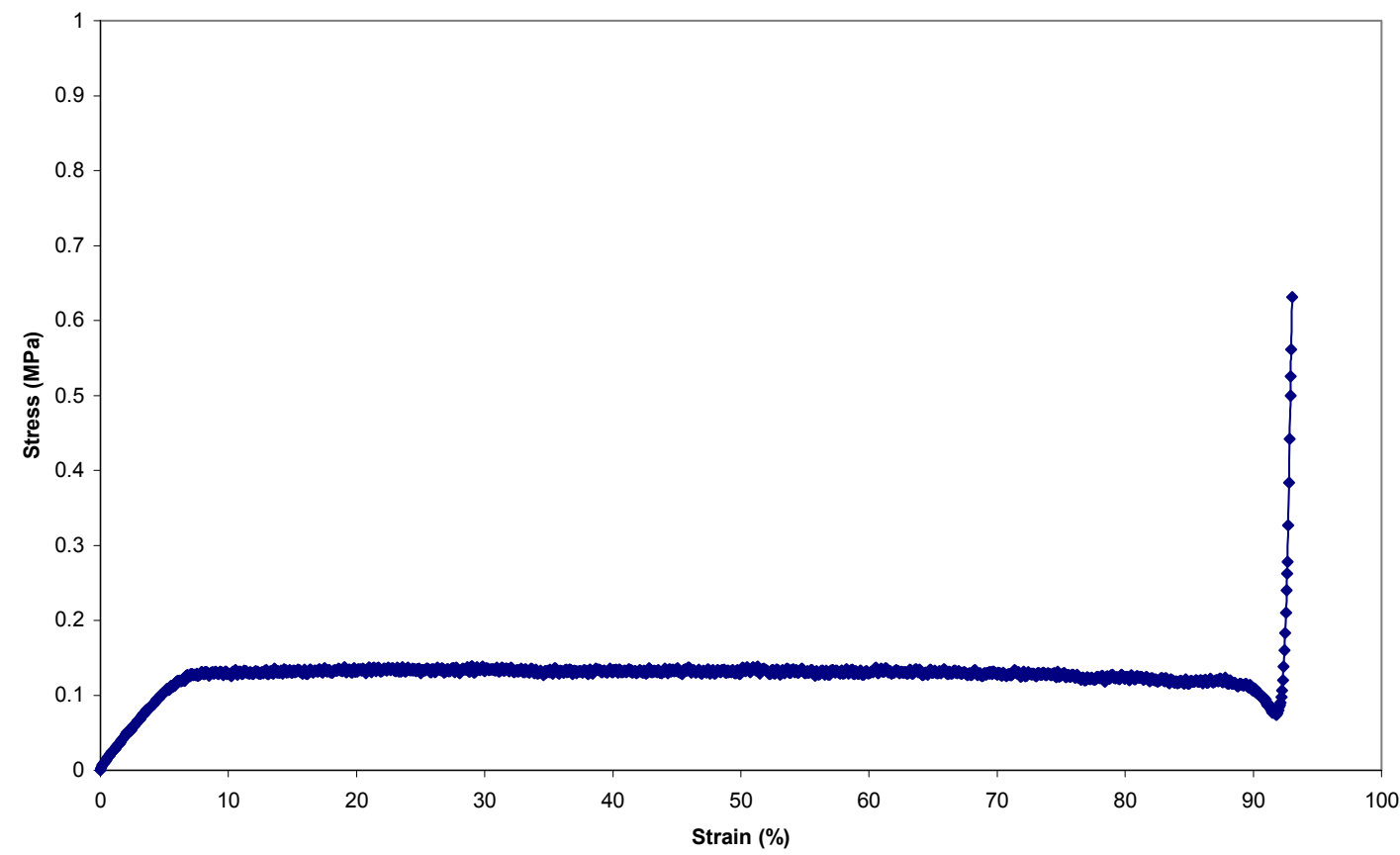

Figure A.14. Stress-Strain Plots for Samples 7 and 8 of Test 2.3. 
2.3.9

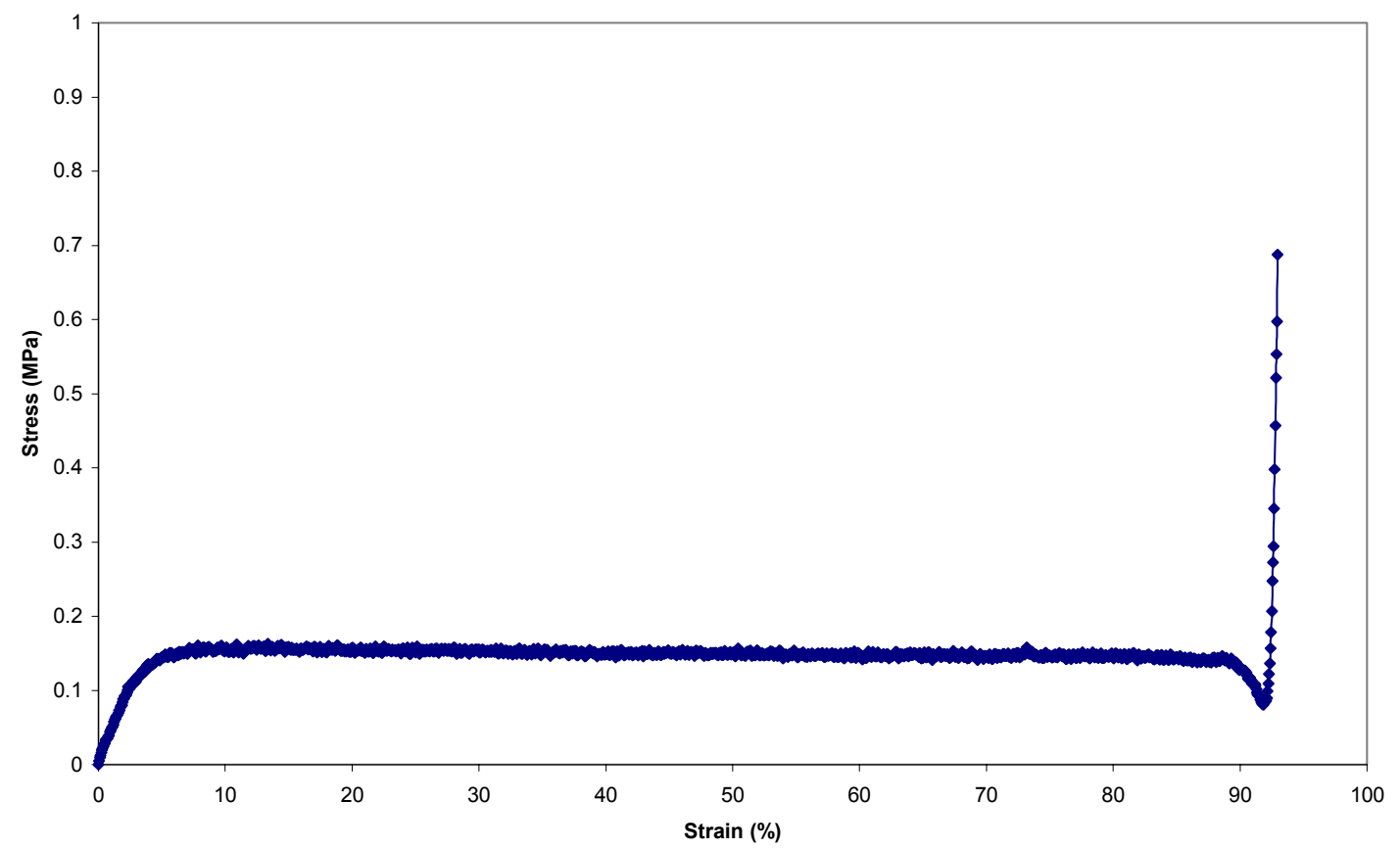

2.3.10

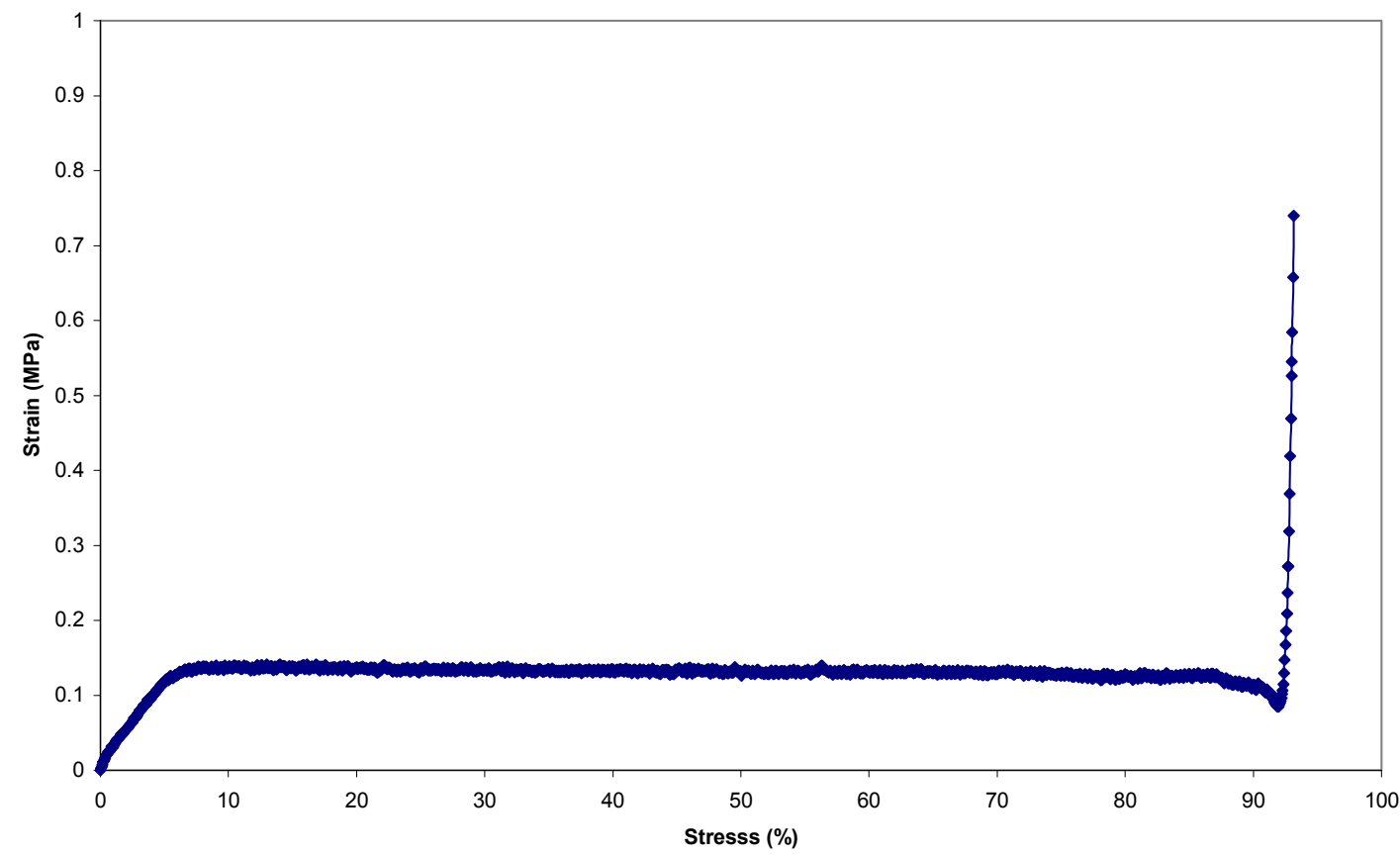

Figure A.15. Stress-Strain Plots for Samples 9 and 10 of Test 2.3. 


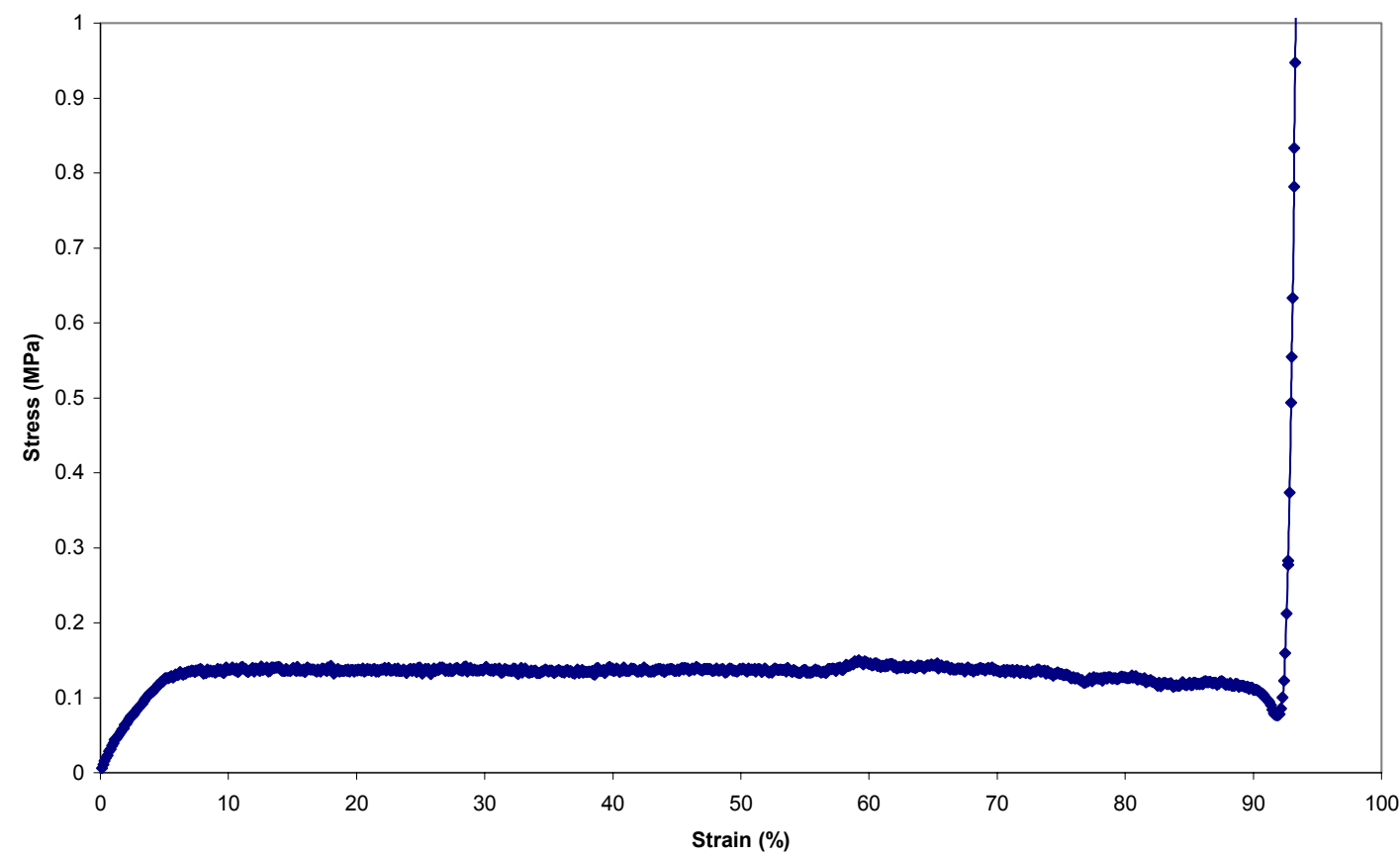

2.4 .2

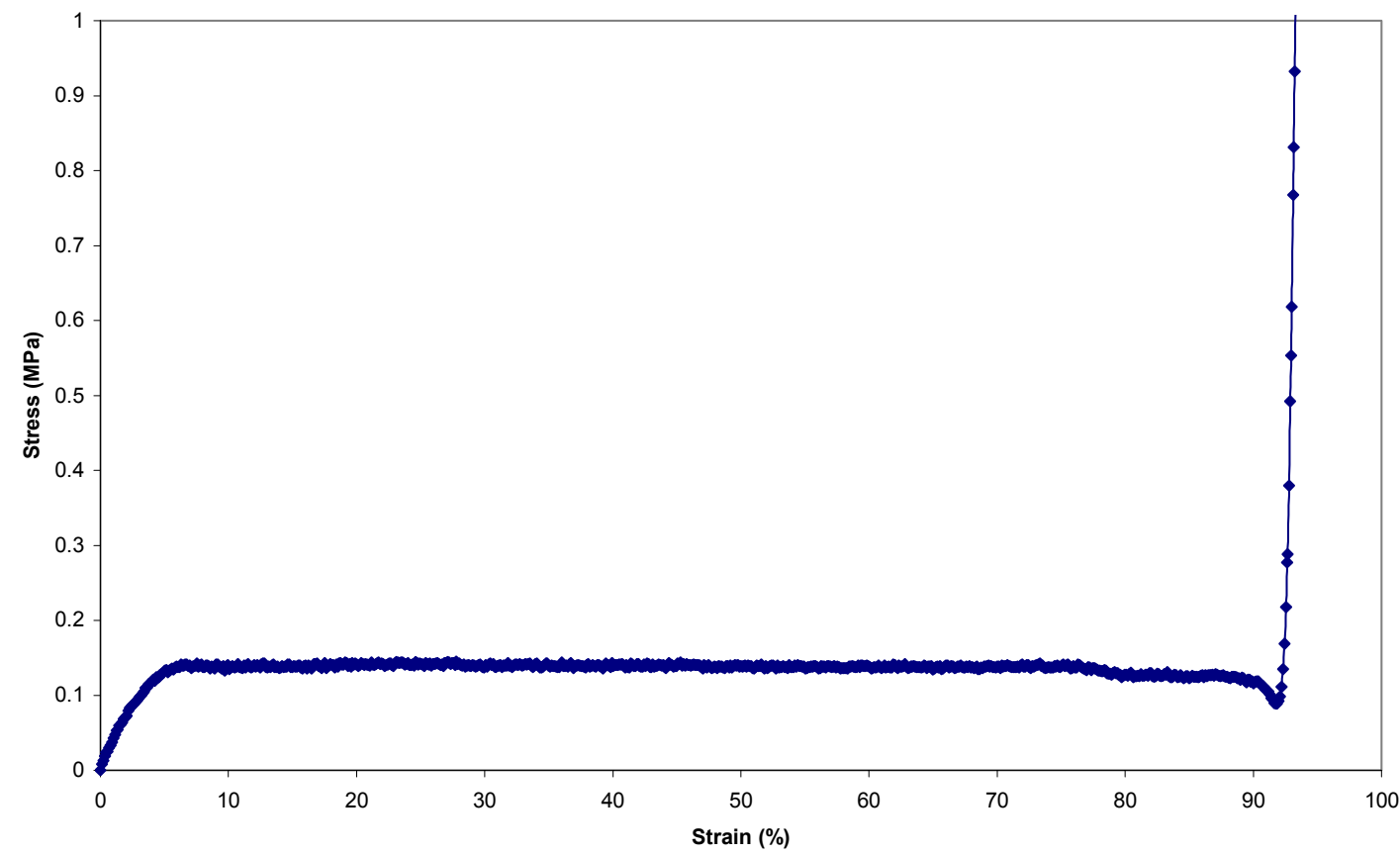

Figure A.16. Stress-Strain Plots for Samples 1 and 2 of Test 2.4. 


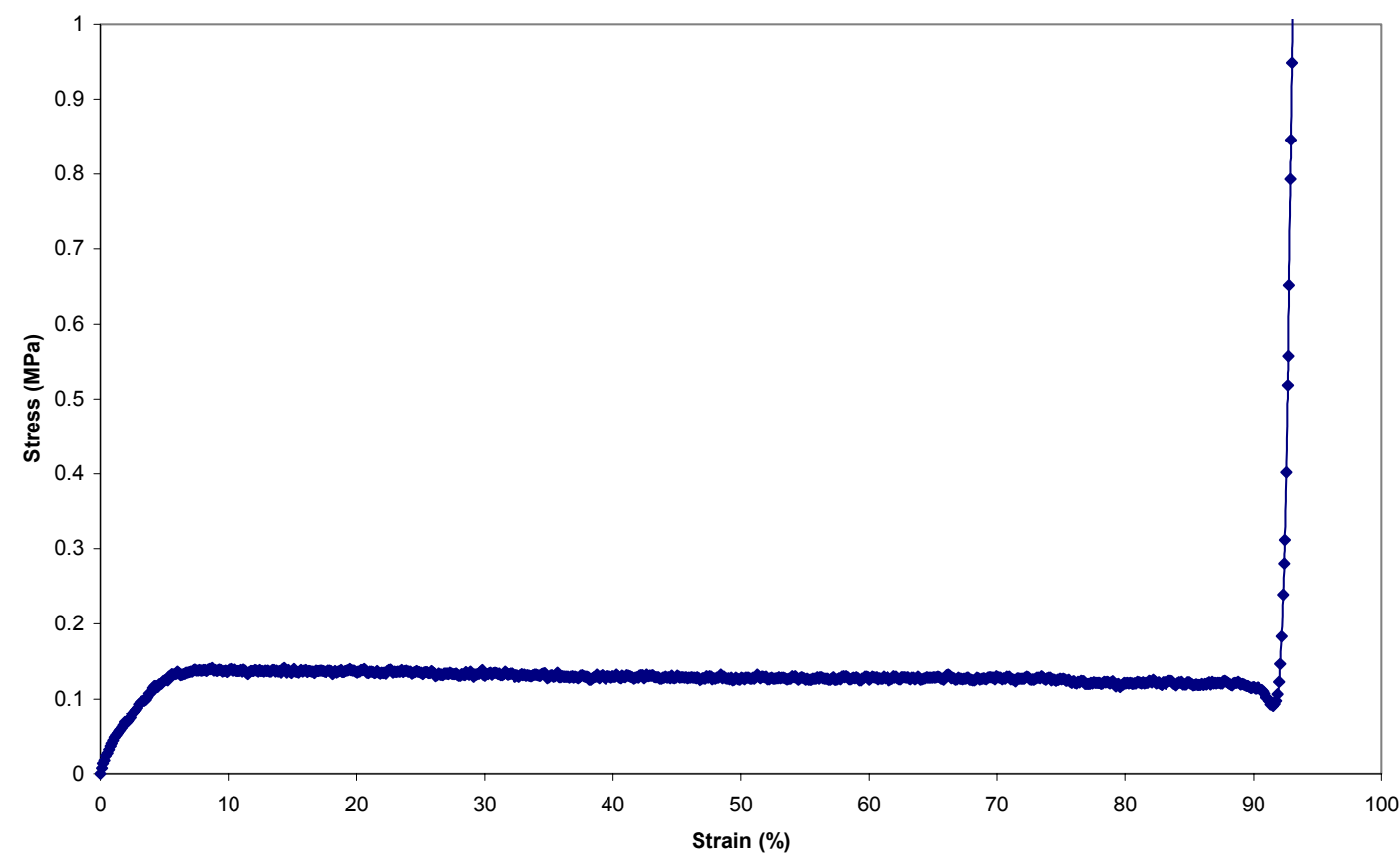

2.4.4

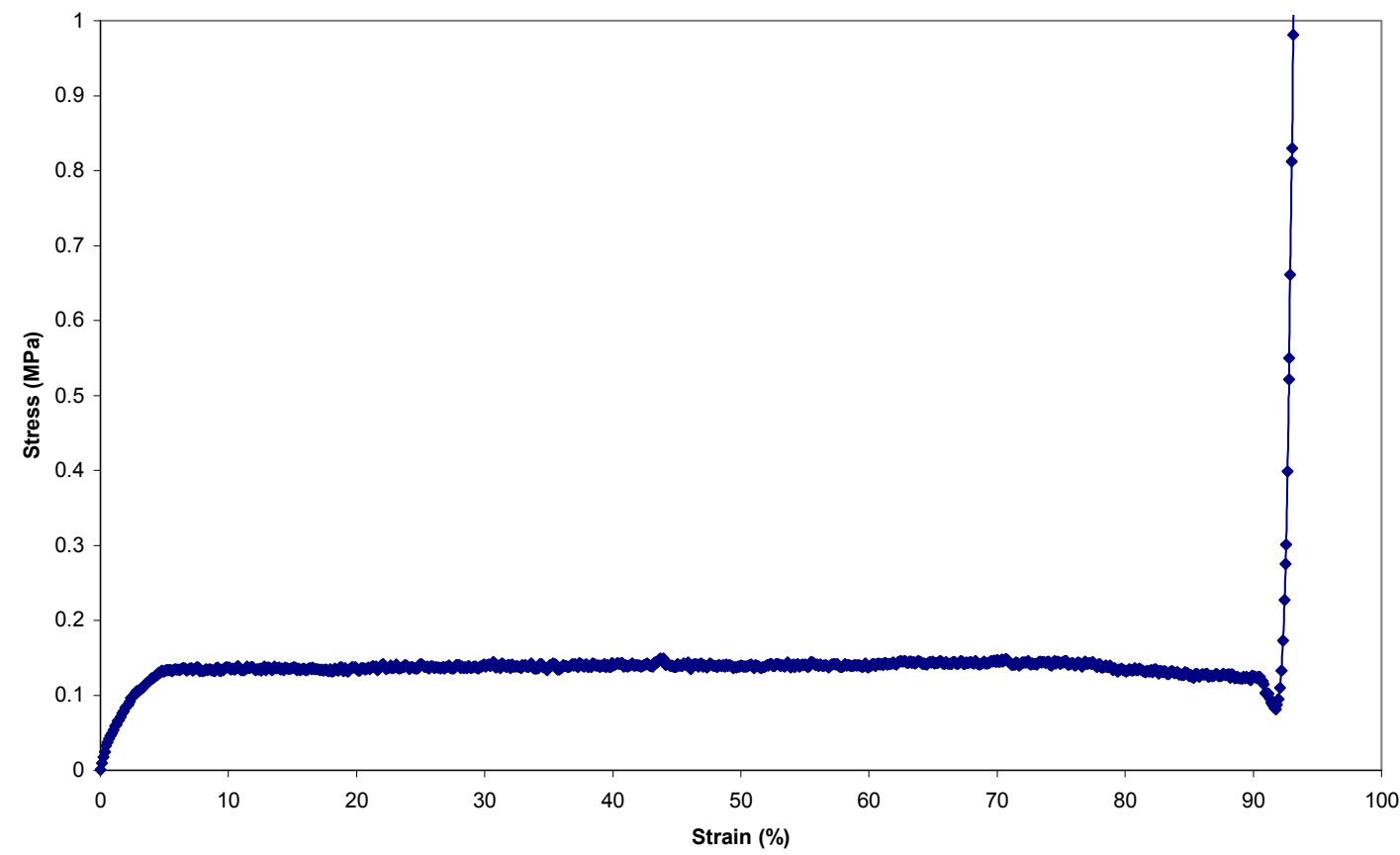

Figure A.17. Stress-Strain Plots for Samples 2 and 3 of Test 2.4. 


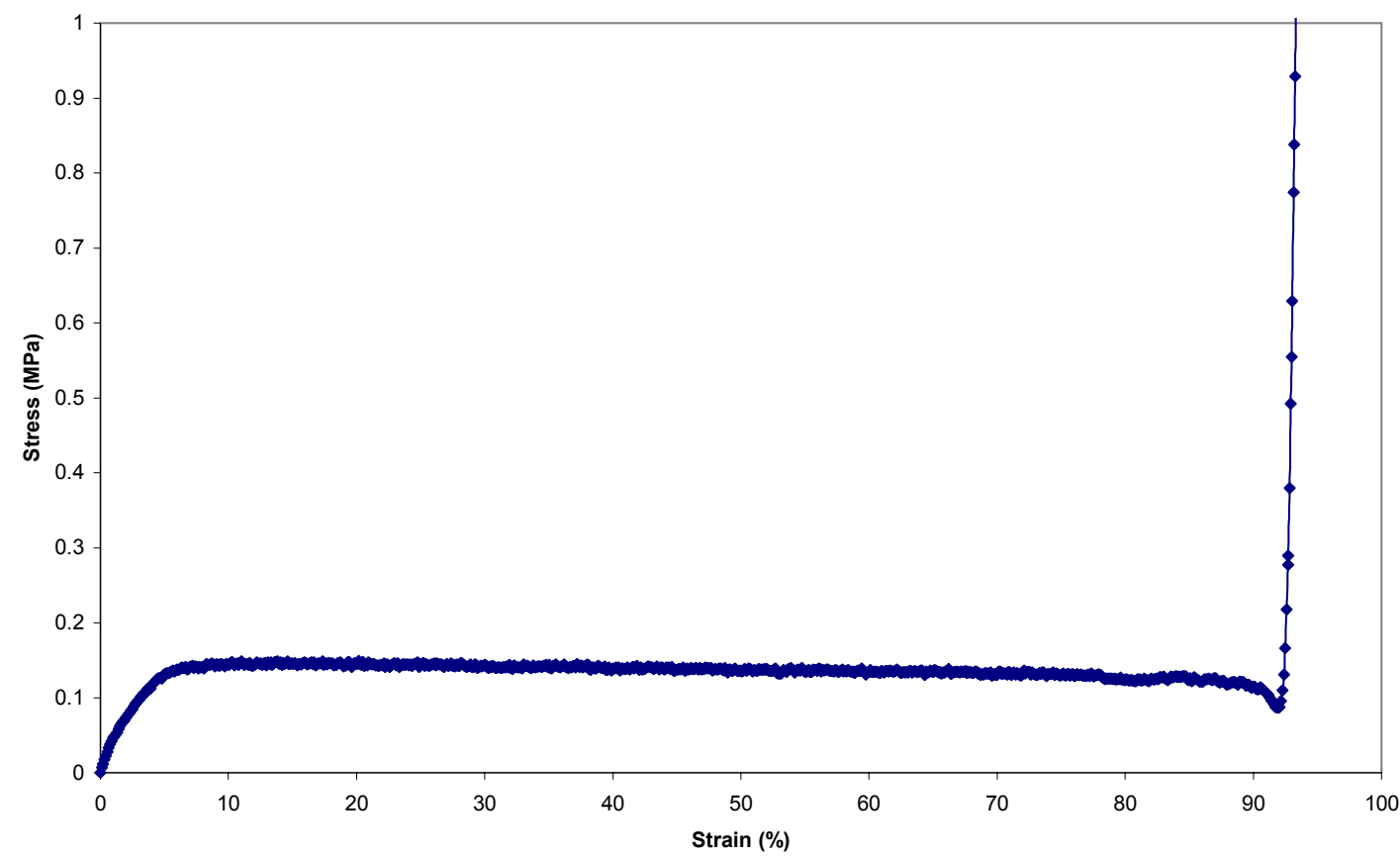

2.4.6

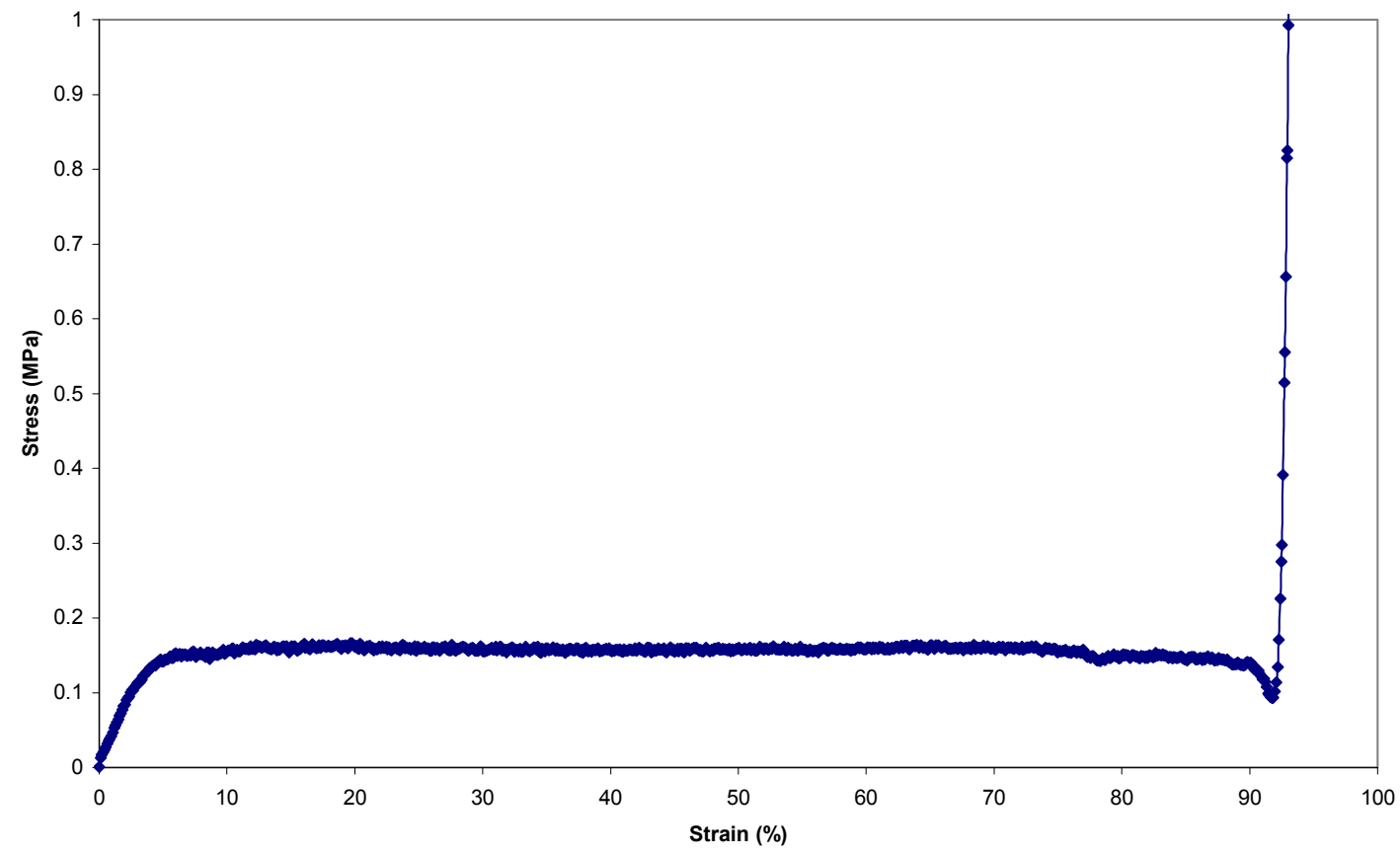

Figure A.18. Stress-Strain Plots for Samples 5 and 6 of Test 2.4. 


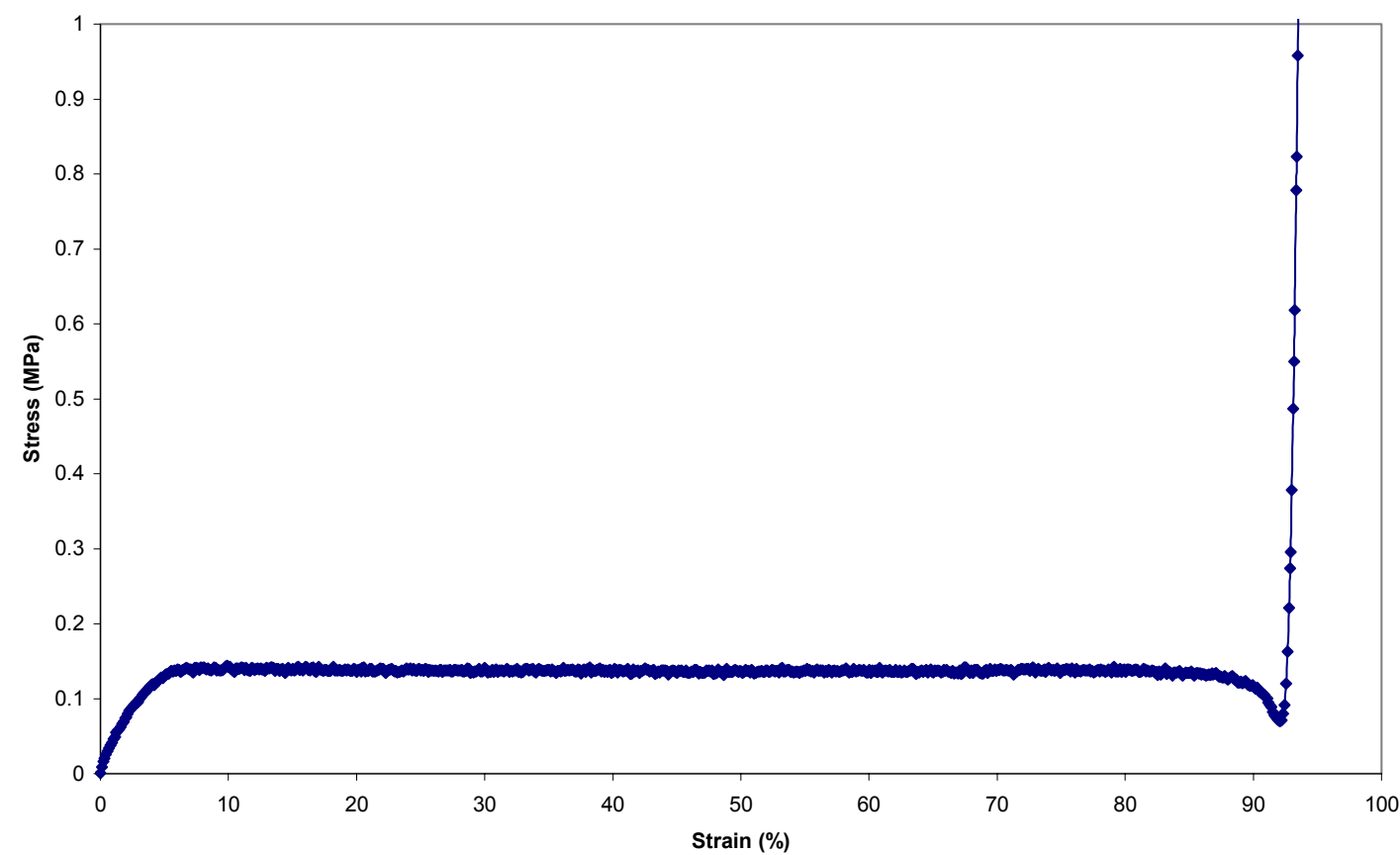

2.4.8

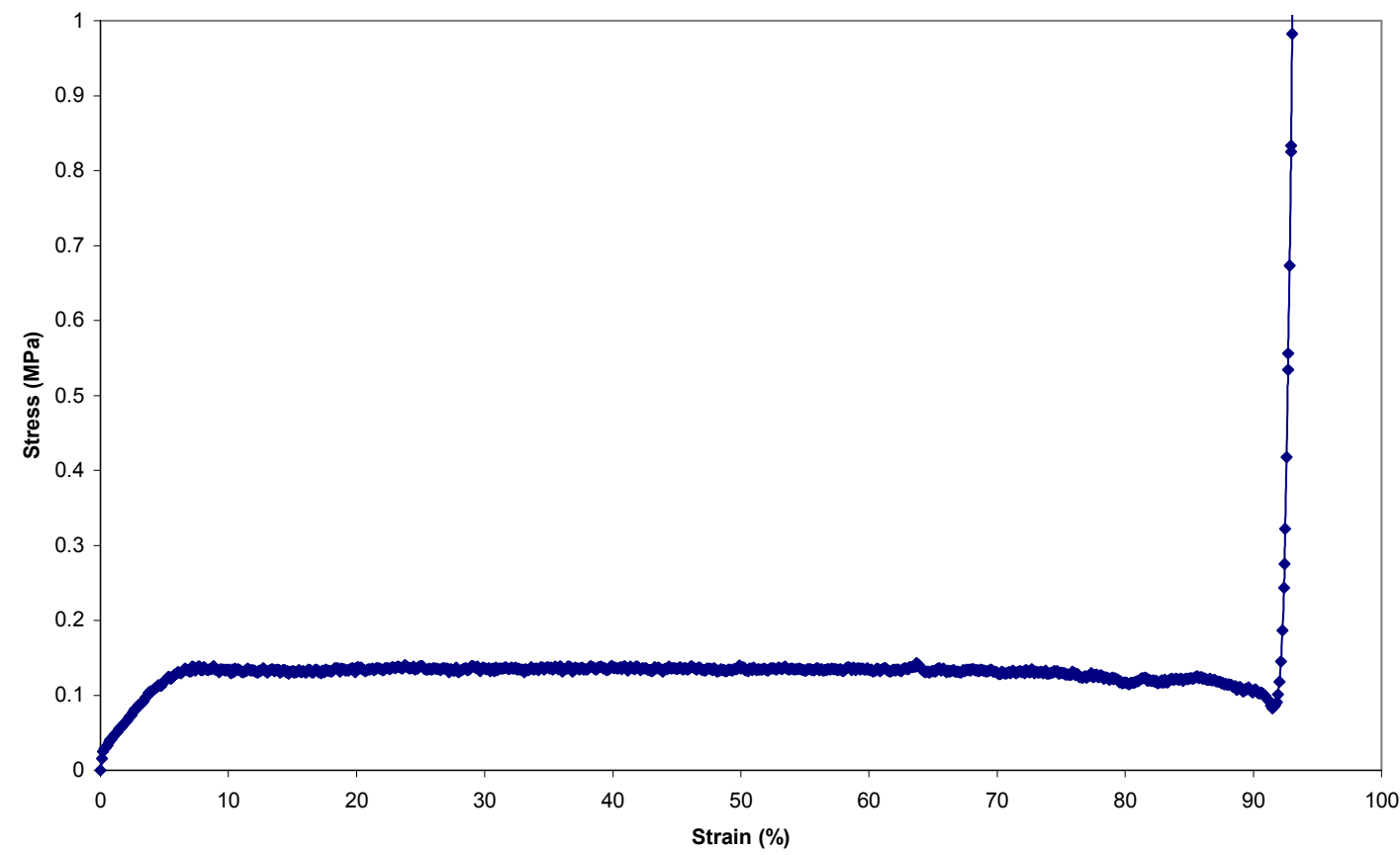

Figure A.19. Stress-Strain Plots for Samples 7 and 8 of Test 2.4. 
2.4.9

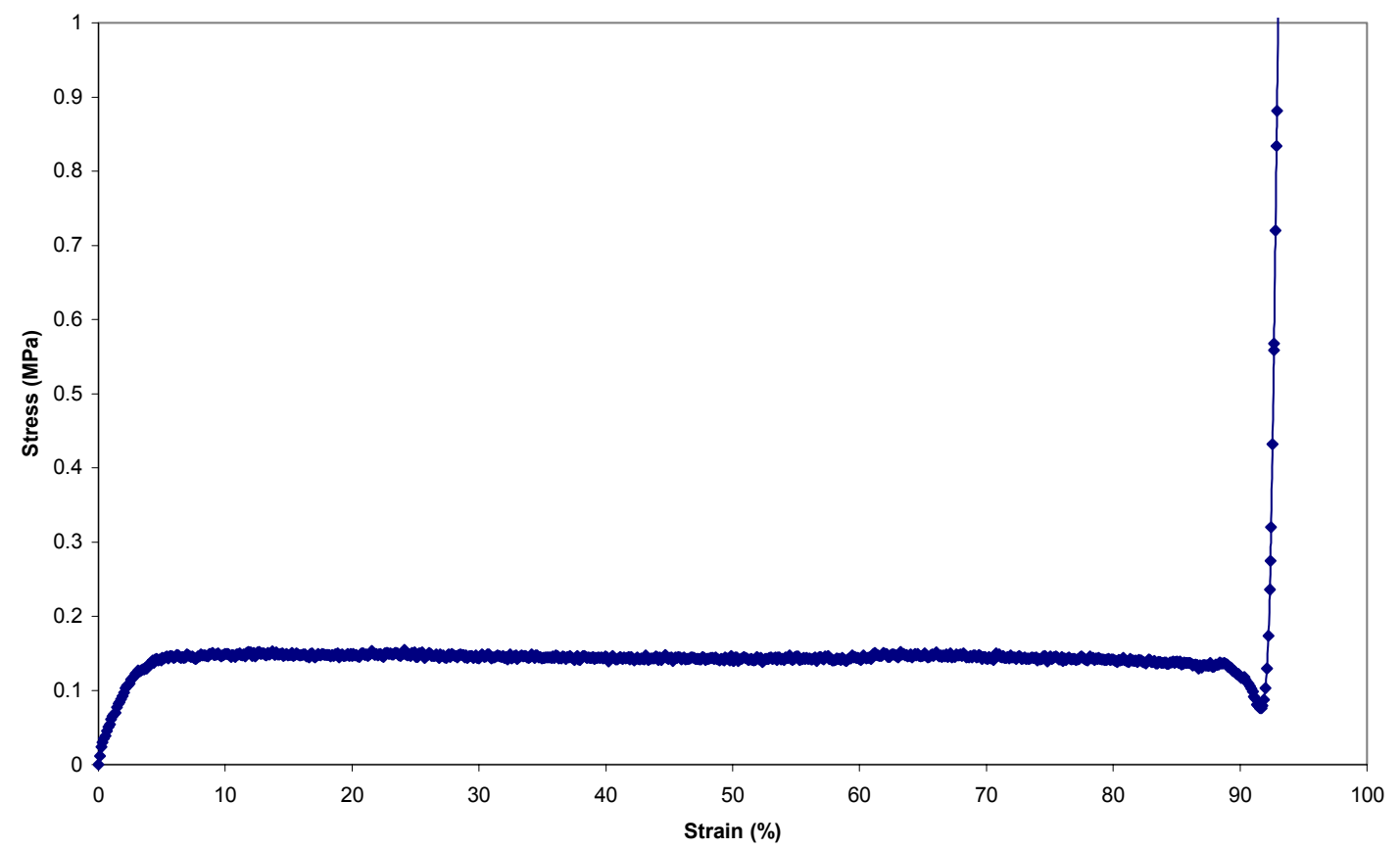

2.4.10

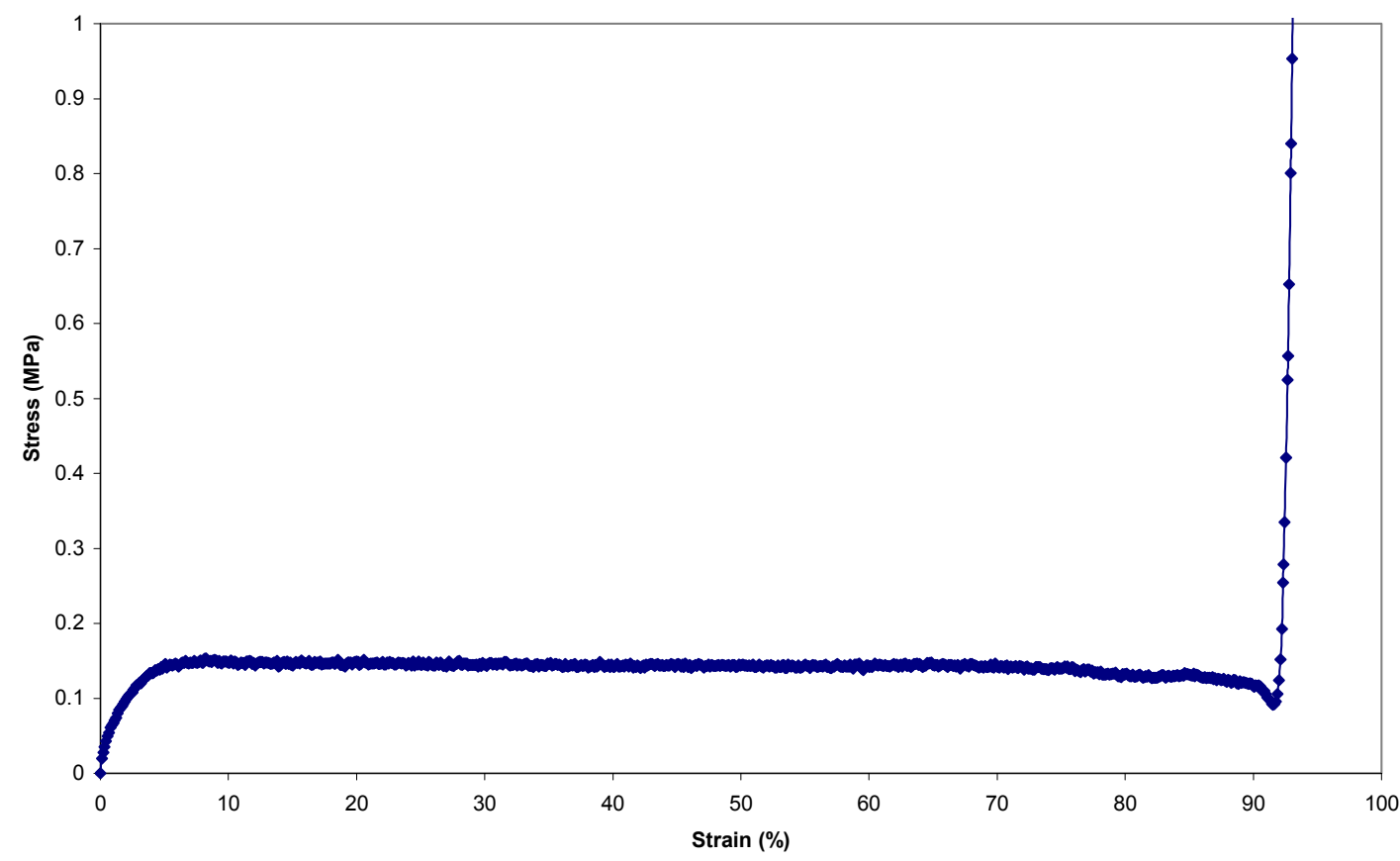

Figure A.20. Stress-Strain Plots for Samples 9 and 10 of Test 2.4. 


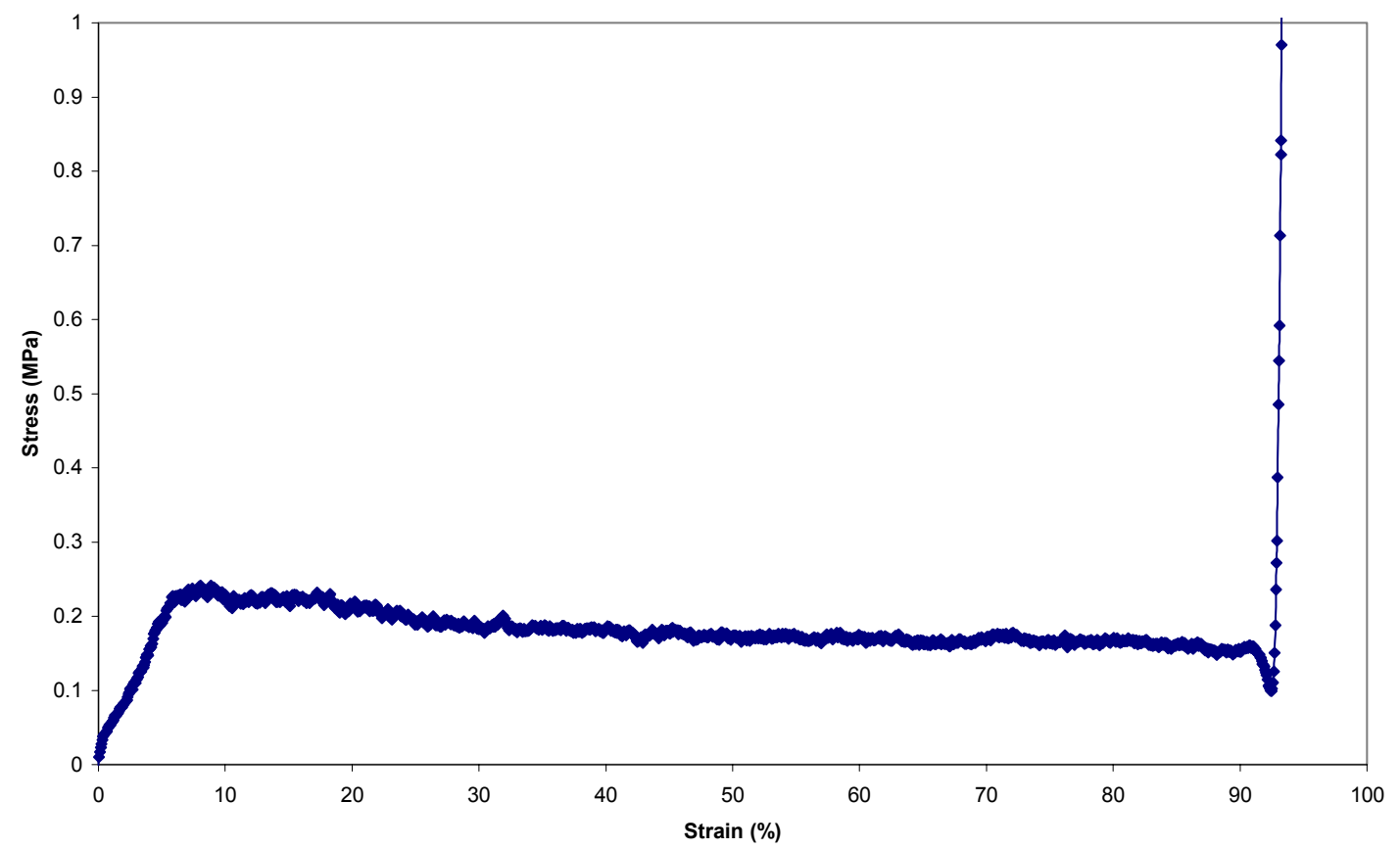

2.5.2

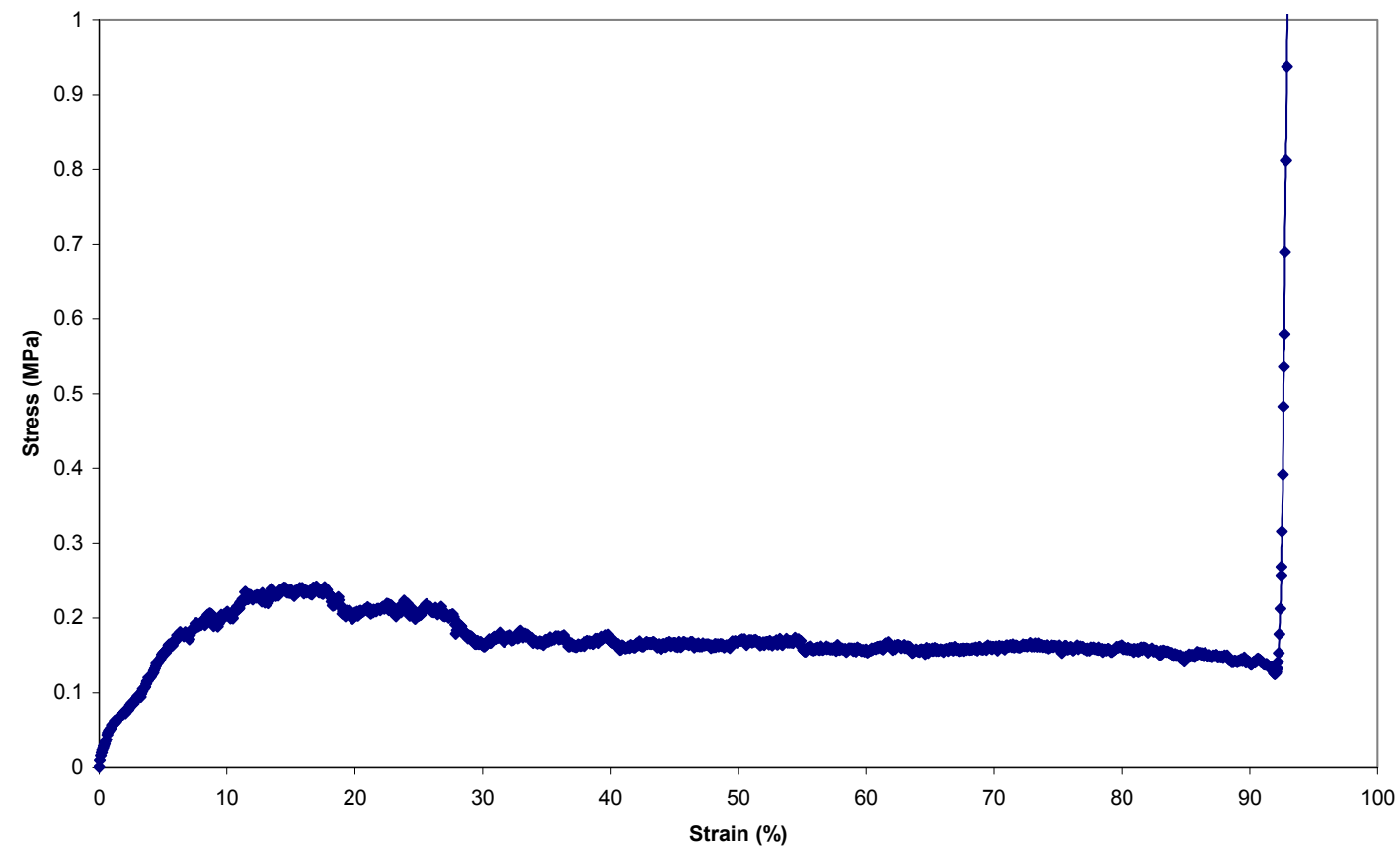

Figure A.21. Stress-Strain Plots for Samples 1 and 2 of Test 2.5. 


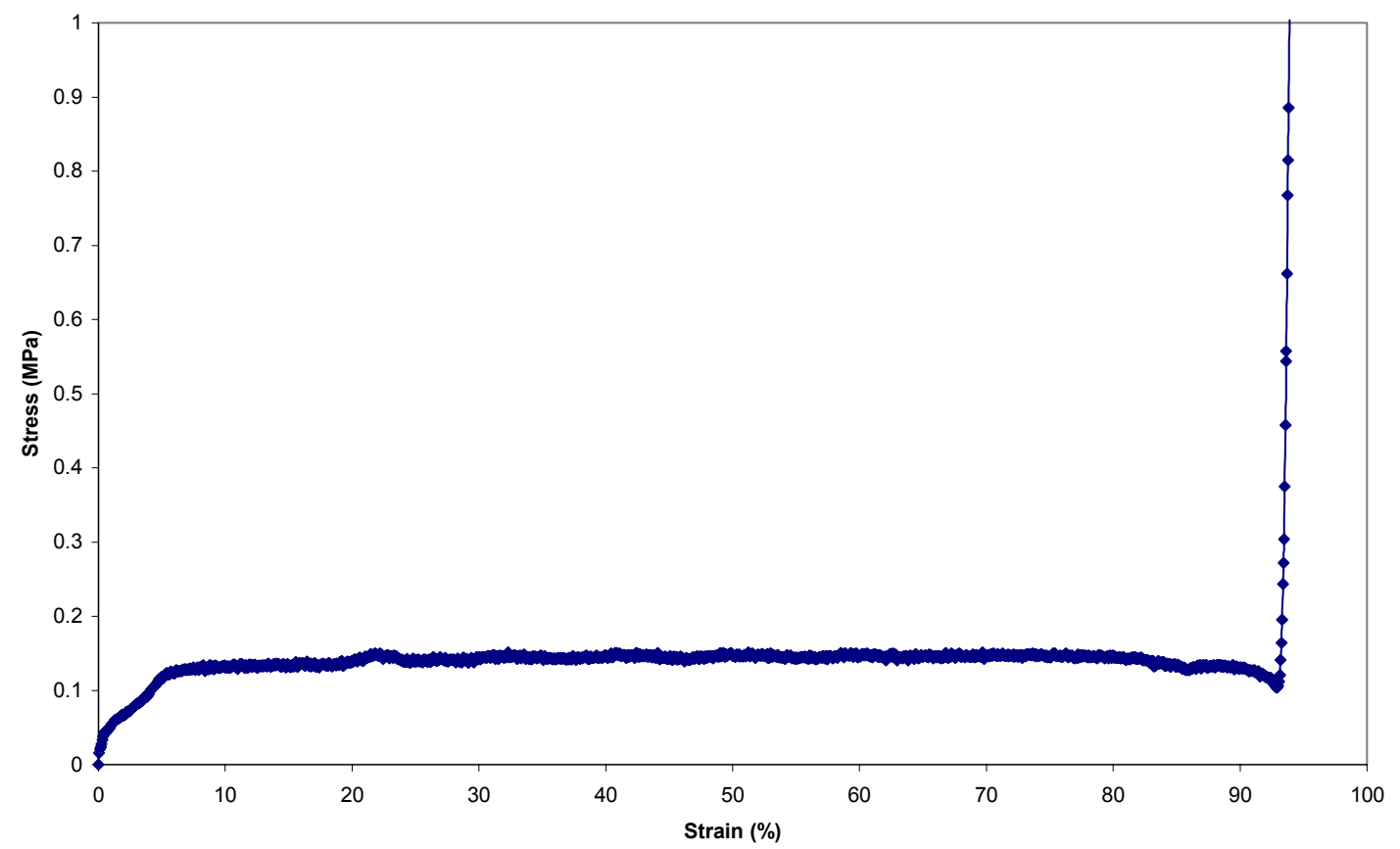

2.5 .4

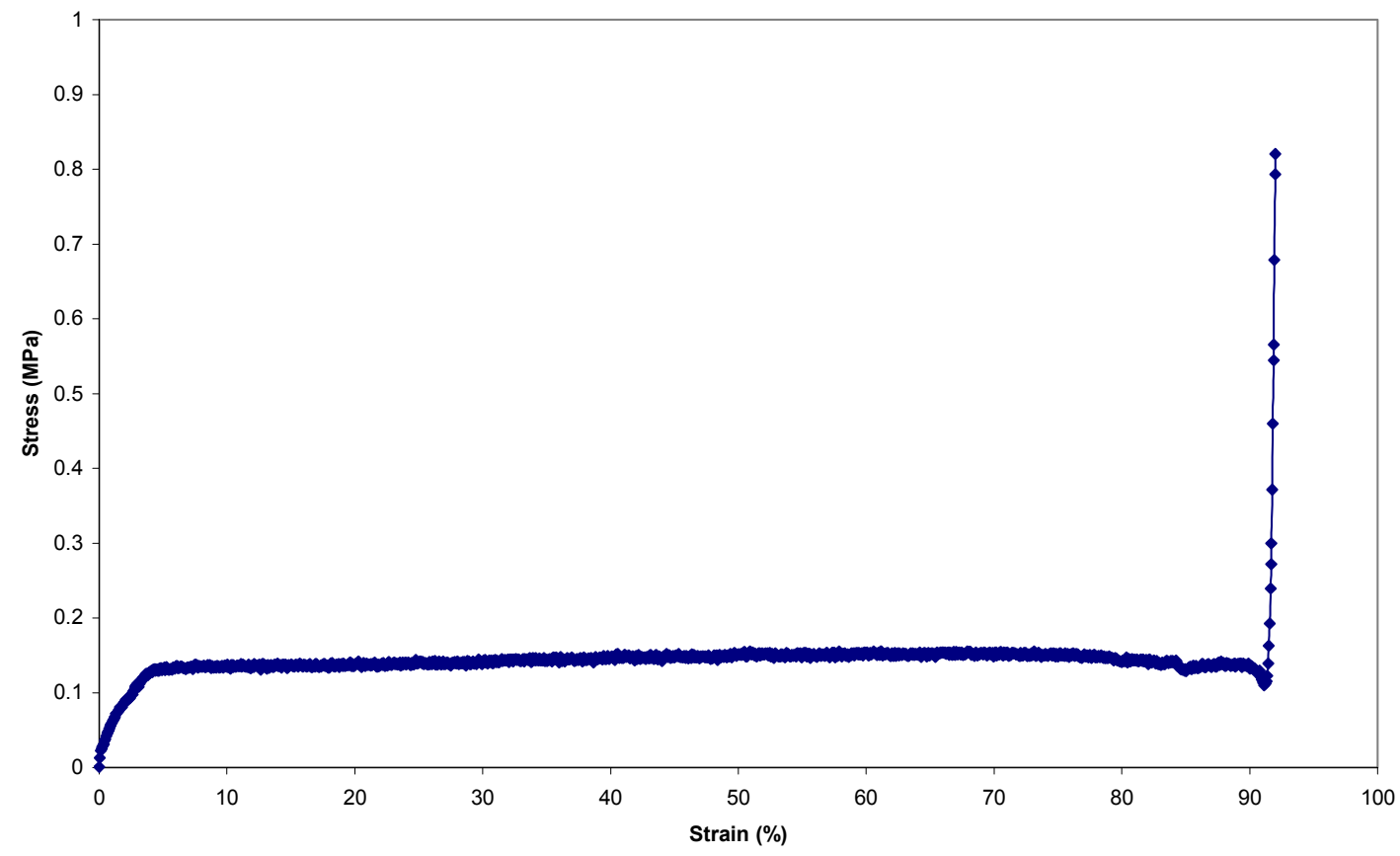

Figure A.22. Stress-Strain Plots for Samples 3 and 4 of Test 2.5. 


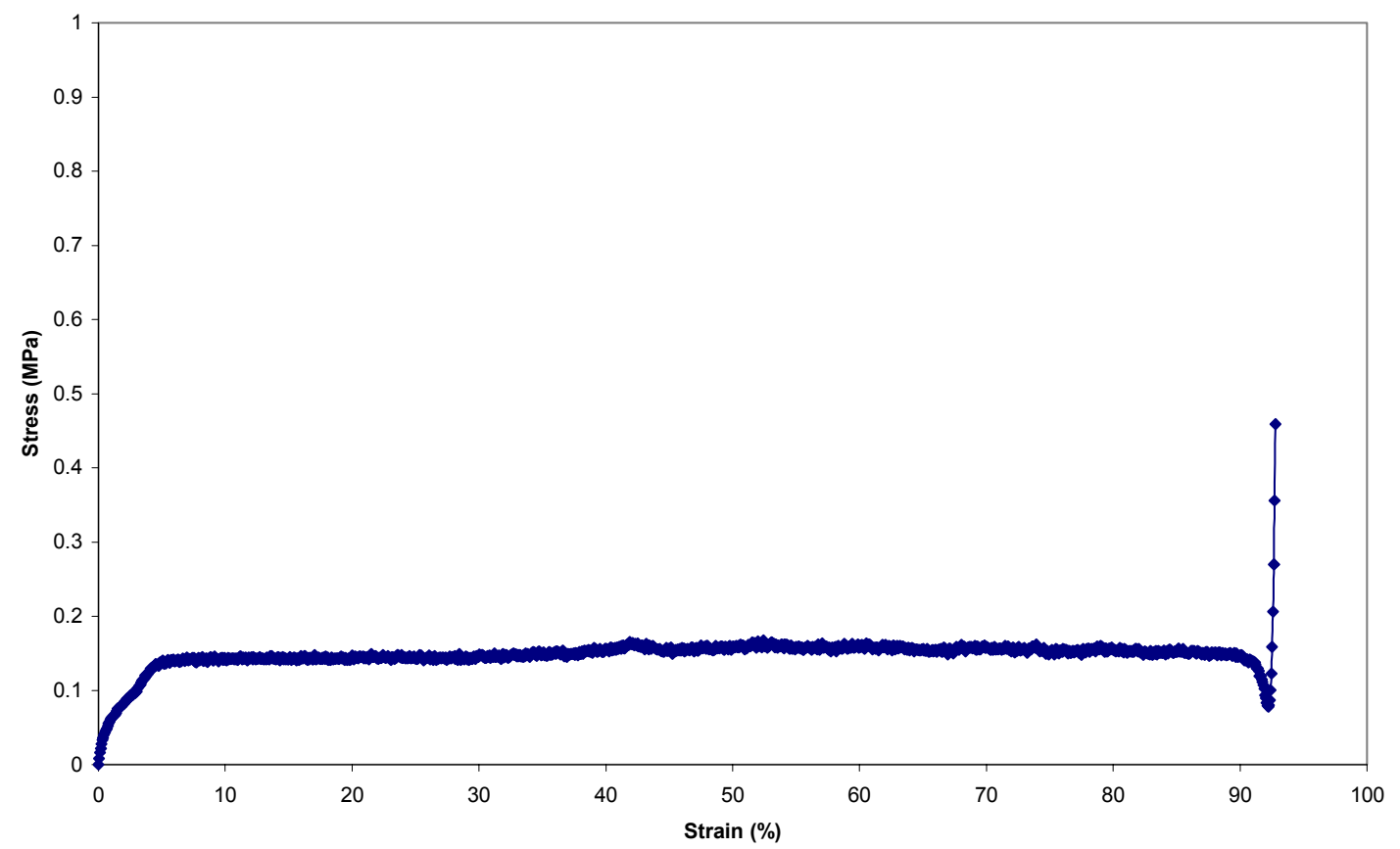

2.5 .6

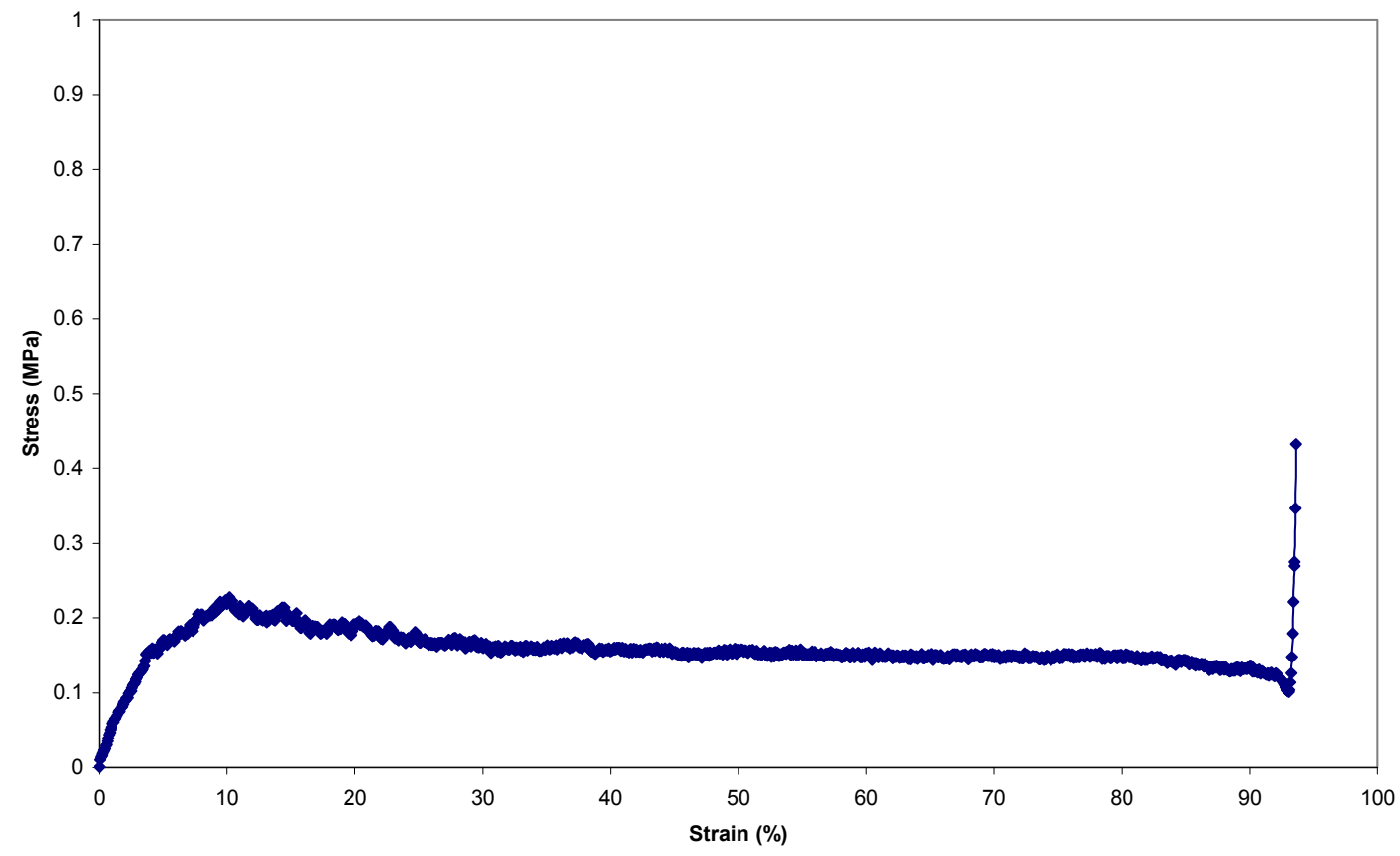

Figure A.23. Stress-Strain Plots for Samples 5 and 6 of Test 2.5. 
2.5.7

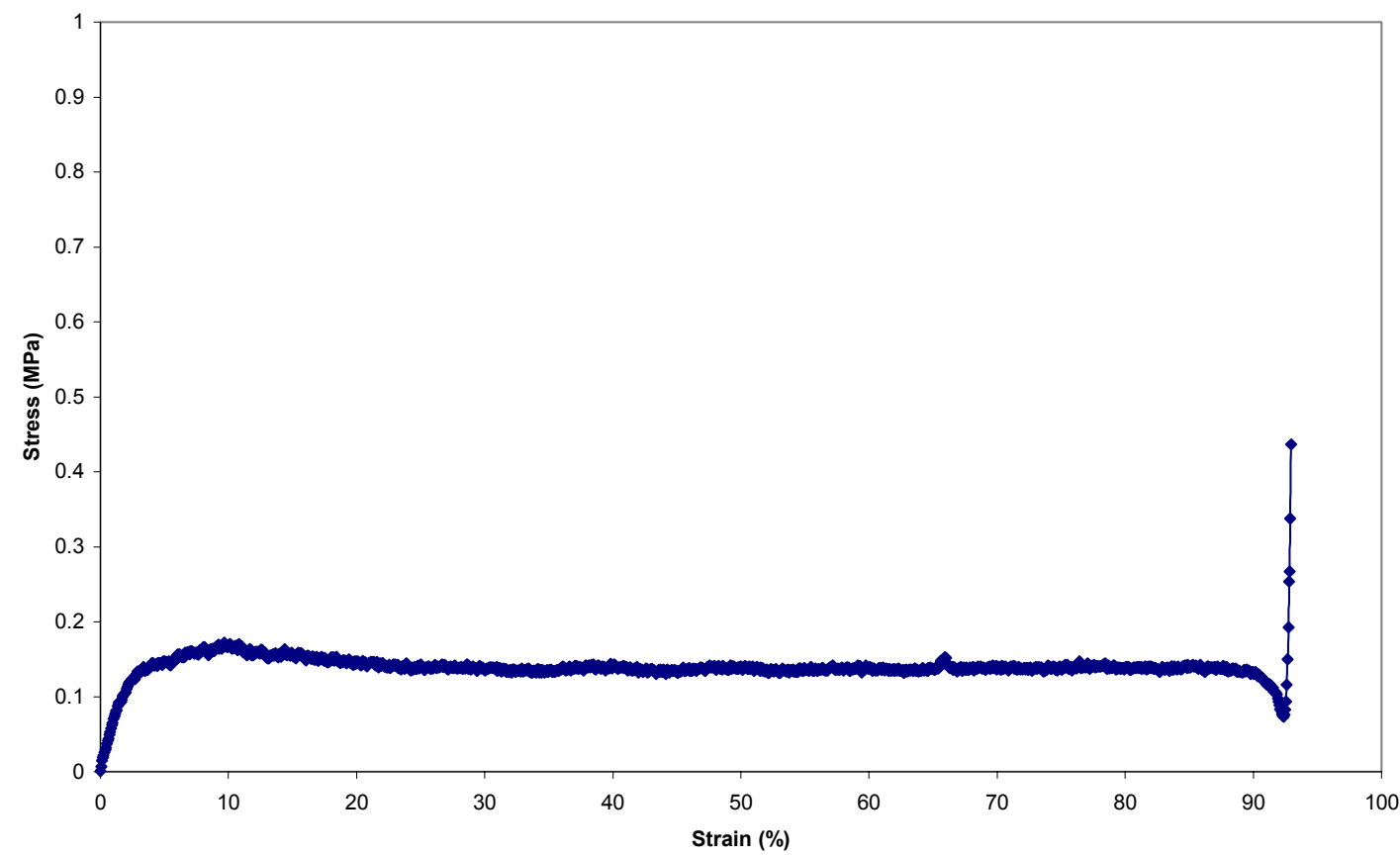

2.5.8

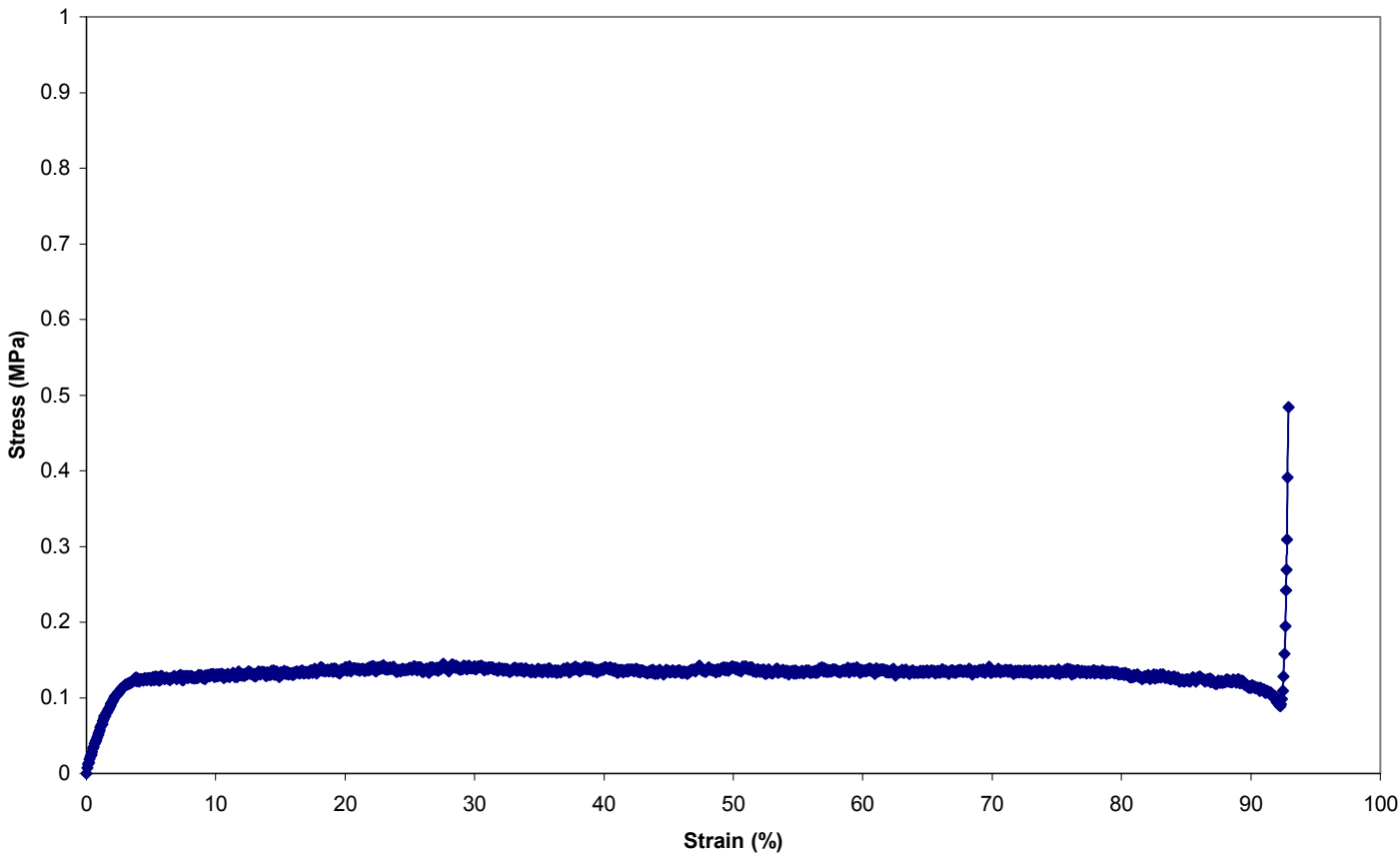

Figure A.24. Stress-Strain Plots for Samples 7 and 8 of Test 2.5. 


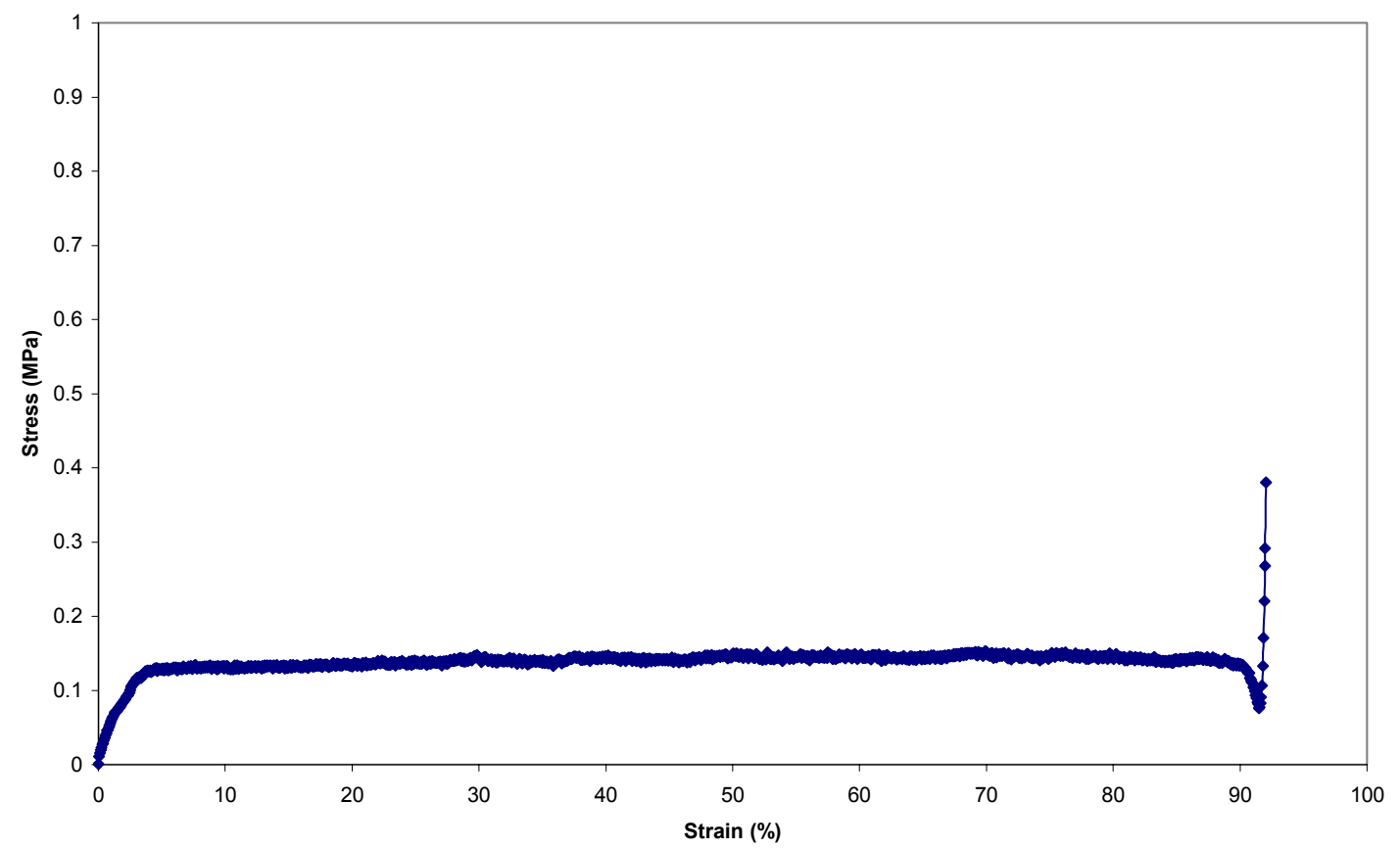

2.5.10

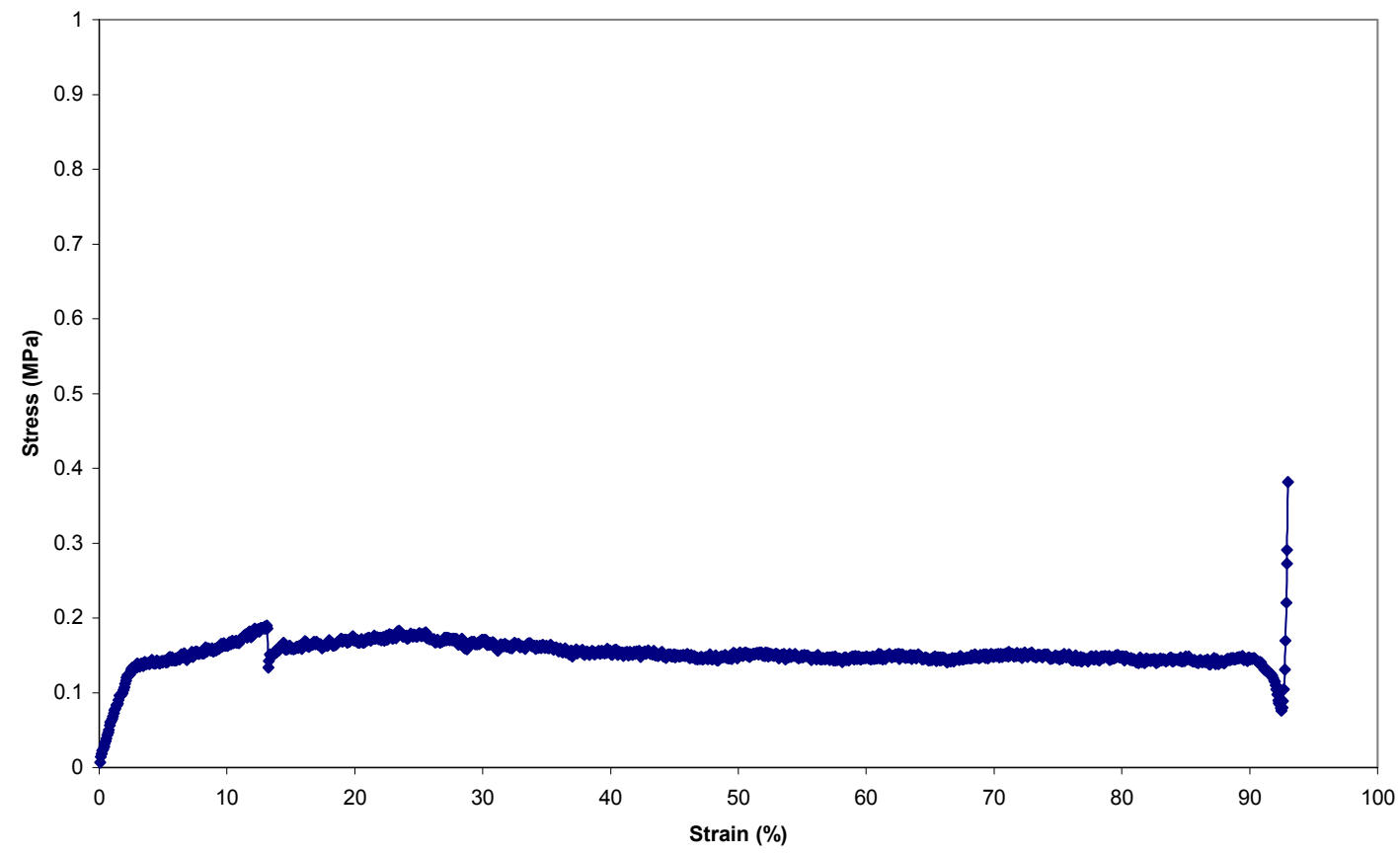

Figure A.25. Stress-Strain Plots for Samples 9 and 10 of Test 2.5. 


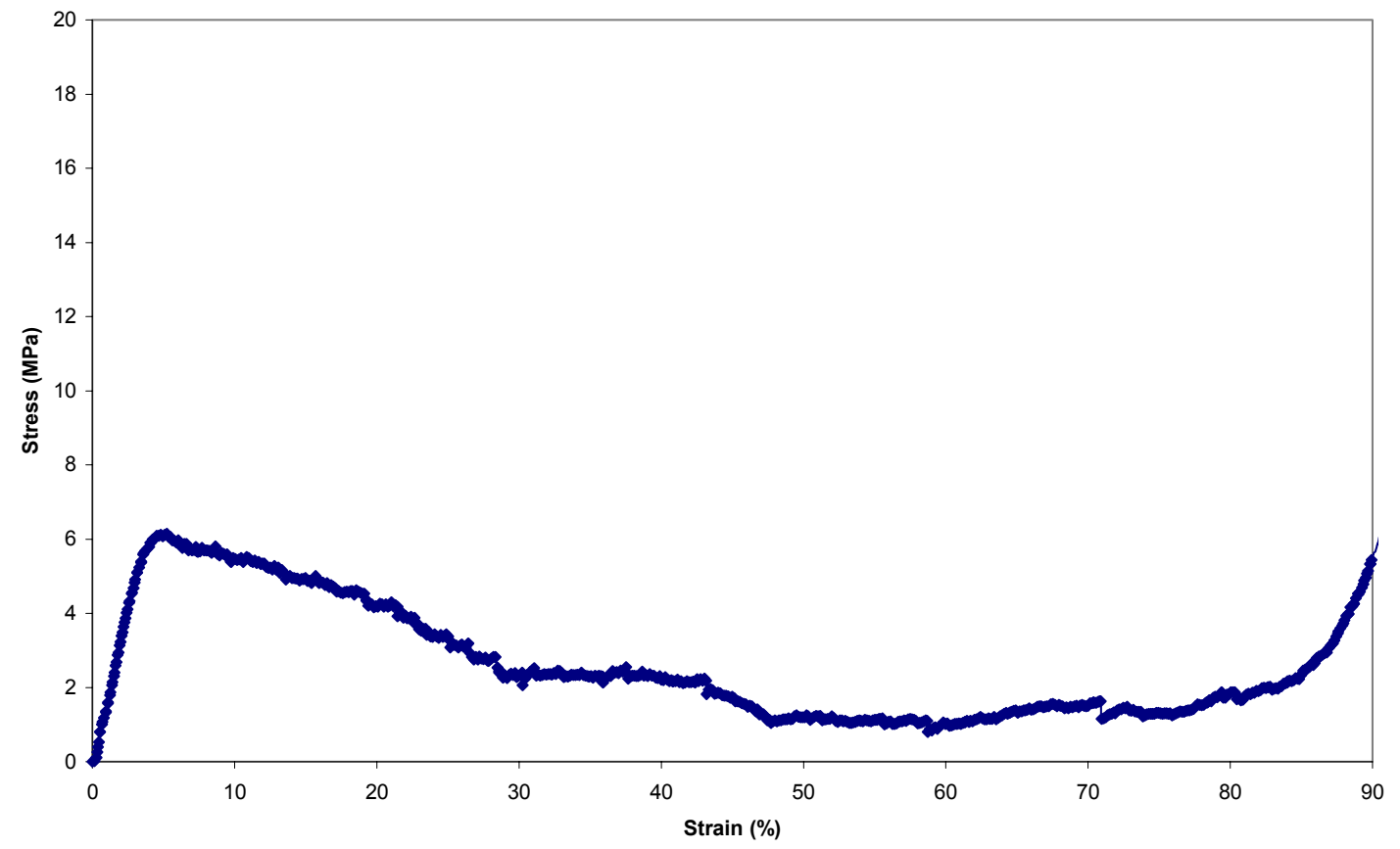

10.1.2

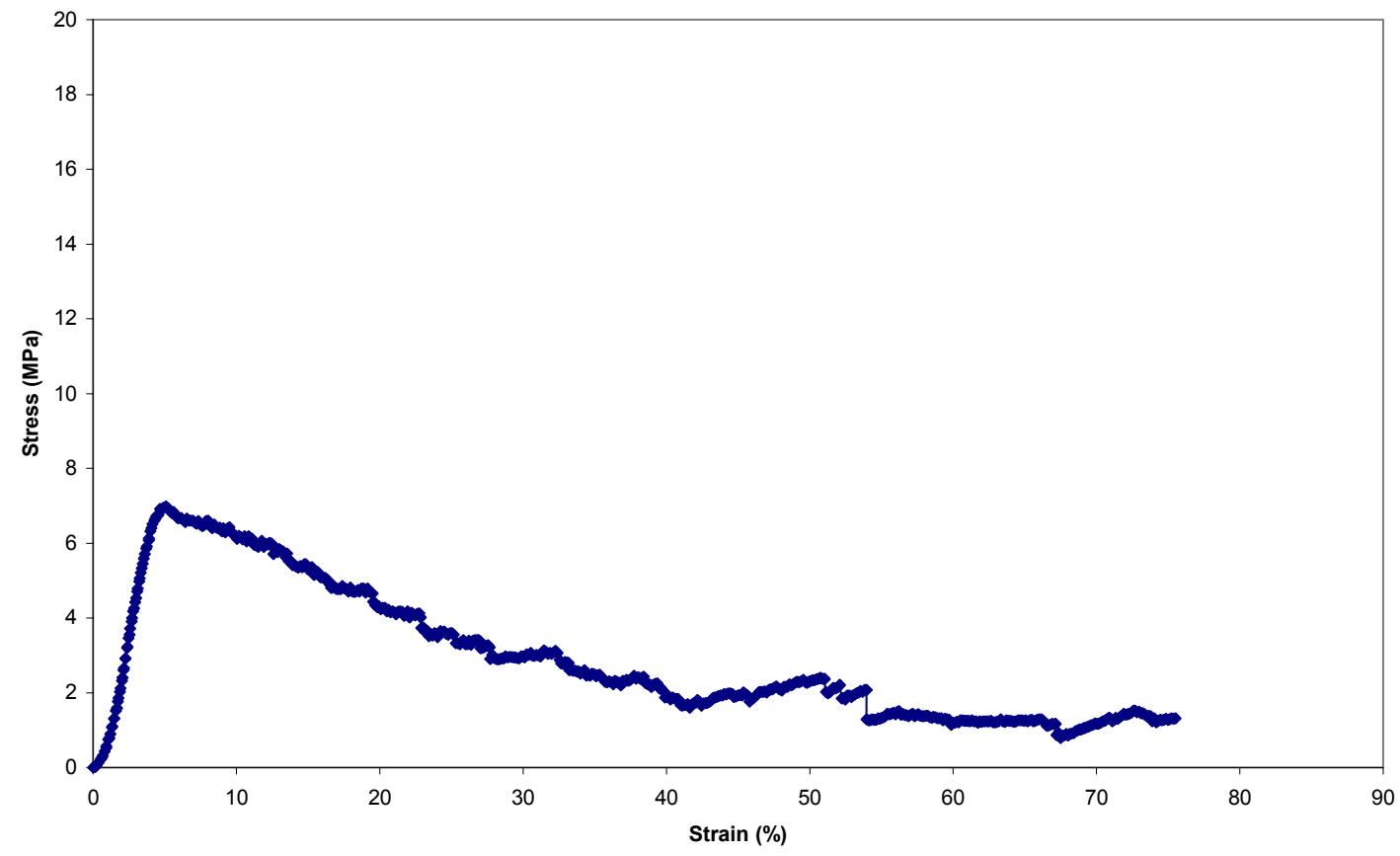

Figure A.26. Stress-Strain Plots for Samples 1 and 2 of Test 10.1. 


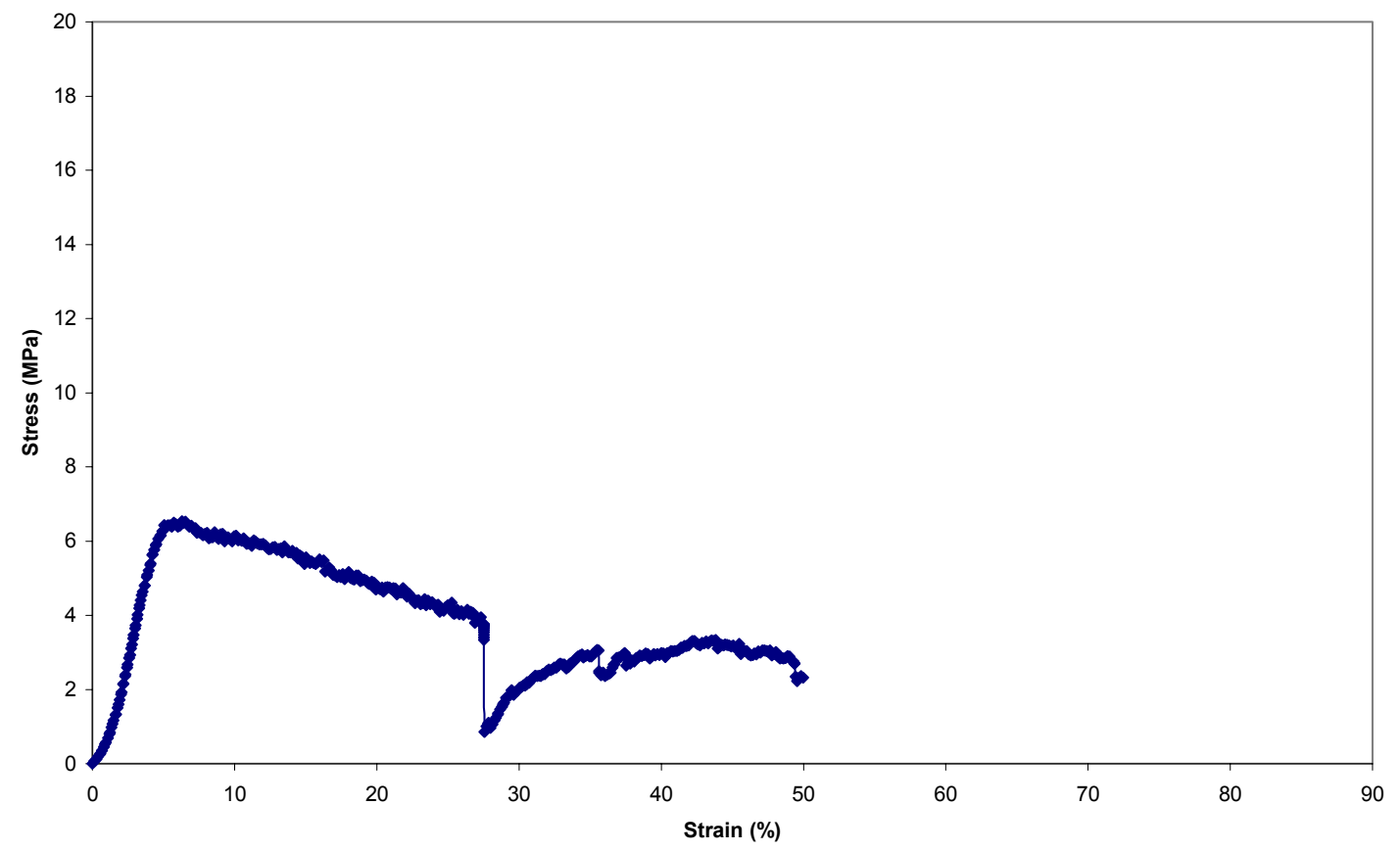

10.1.4

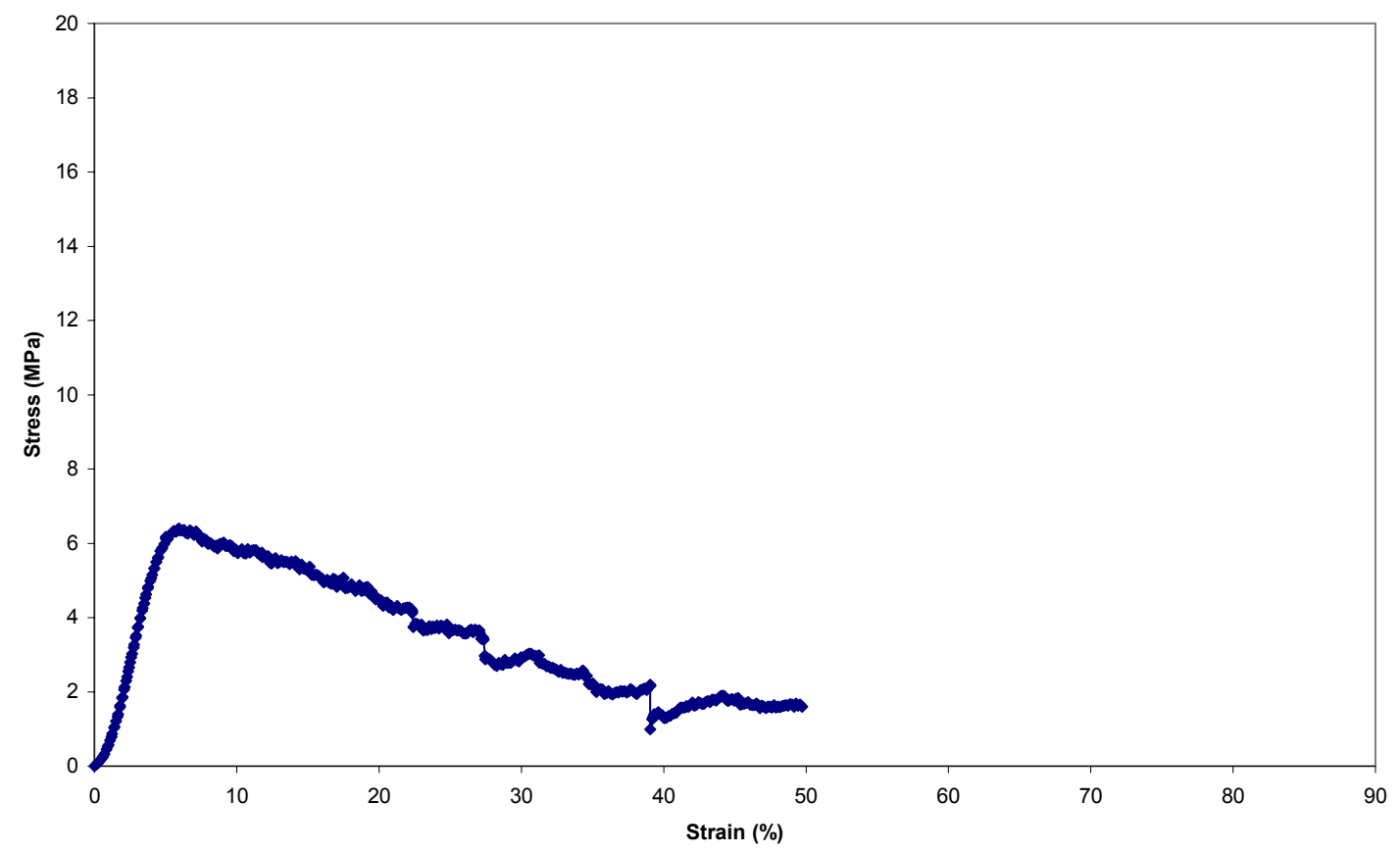

Figure A.27. Stress-Strain Plots for Samples 3 and 4 of Test 10.1. 


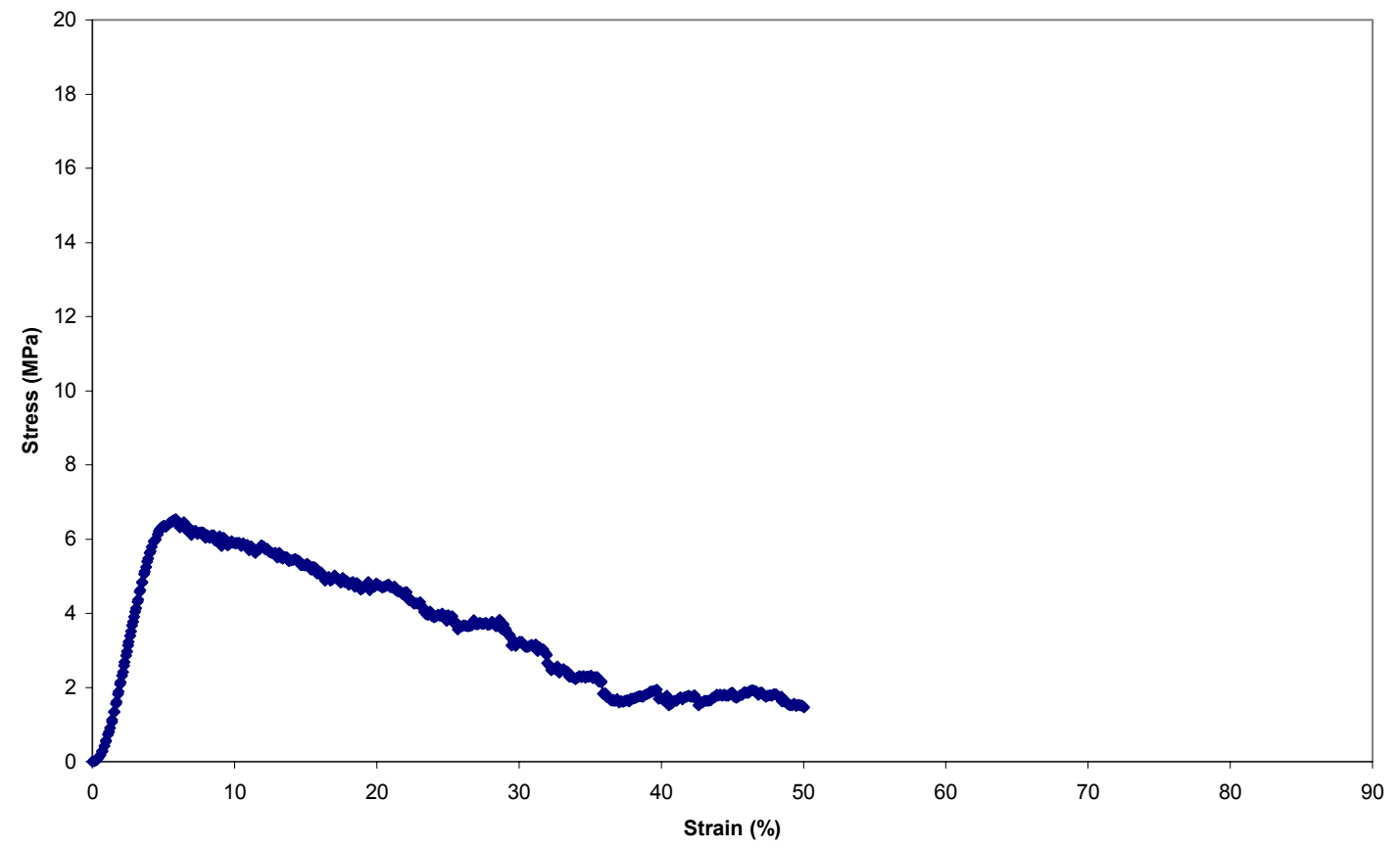

10.1.6

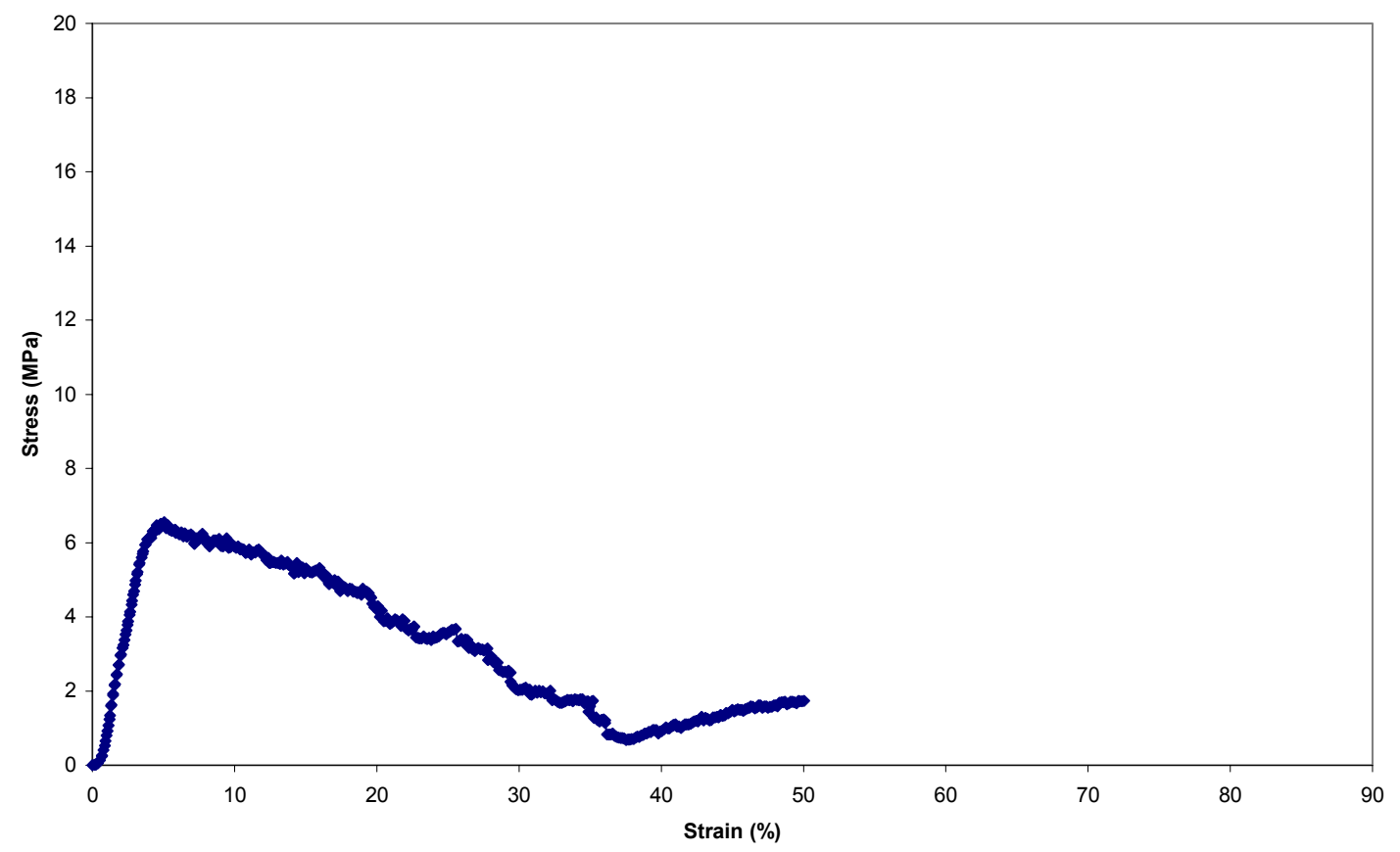

Figure A.28. Stress-Strain Plots for Samples 5 and 6 of Test 10.1. 


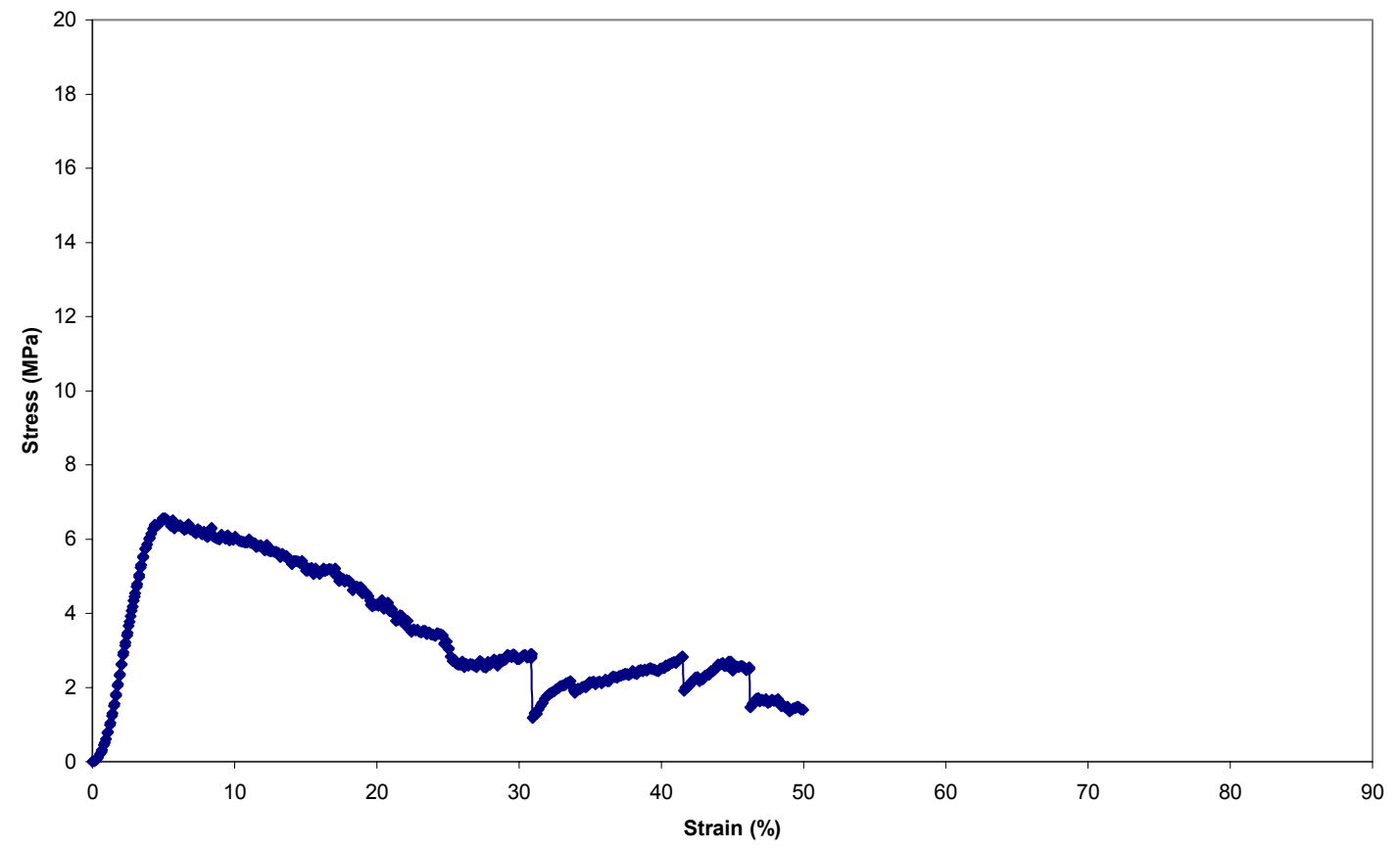

10.1 .8

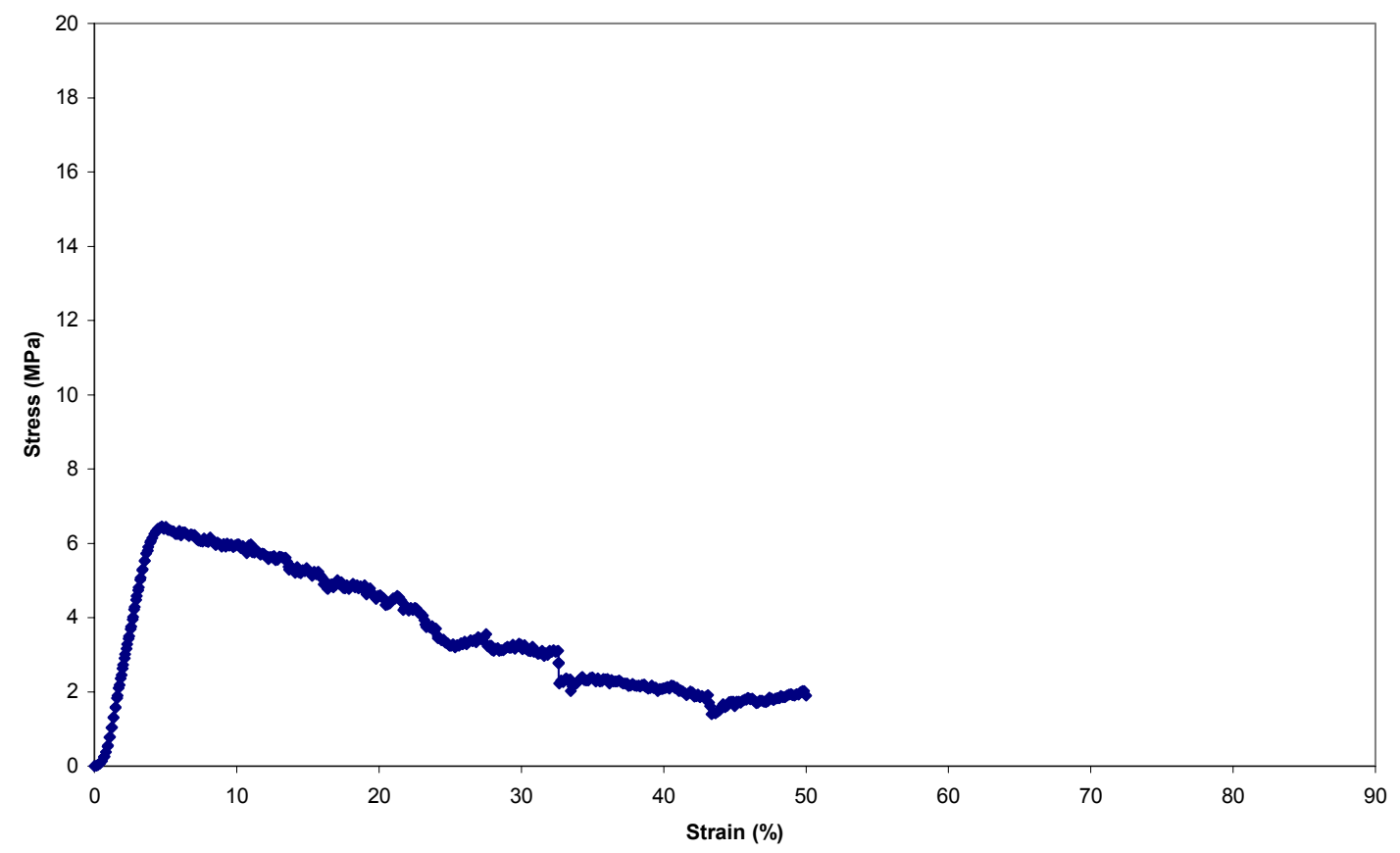

Figure A.29. Stress-Strain Plots for Samples 7 and 8 of Test 10.1. 


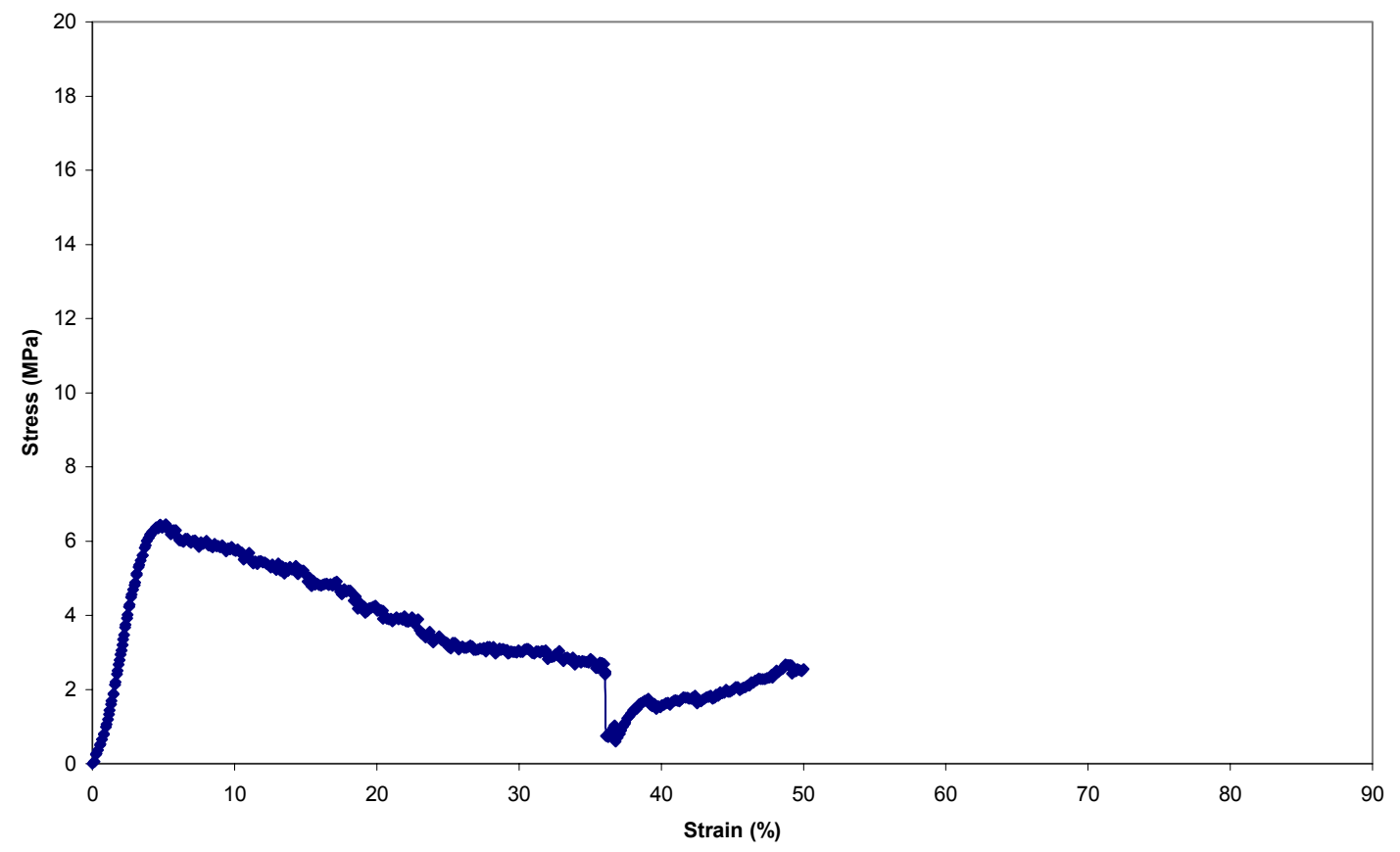

10.1.10

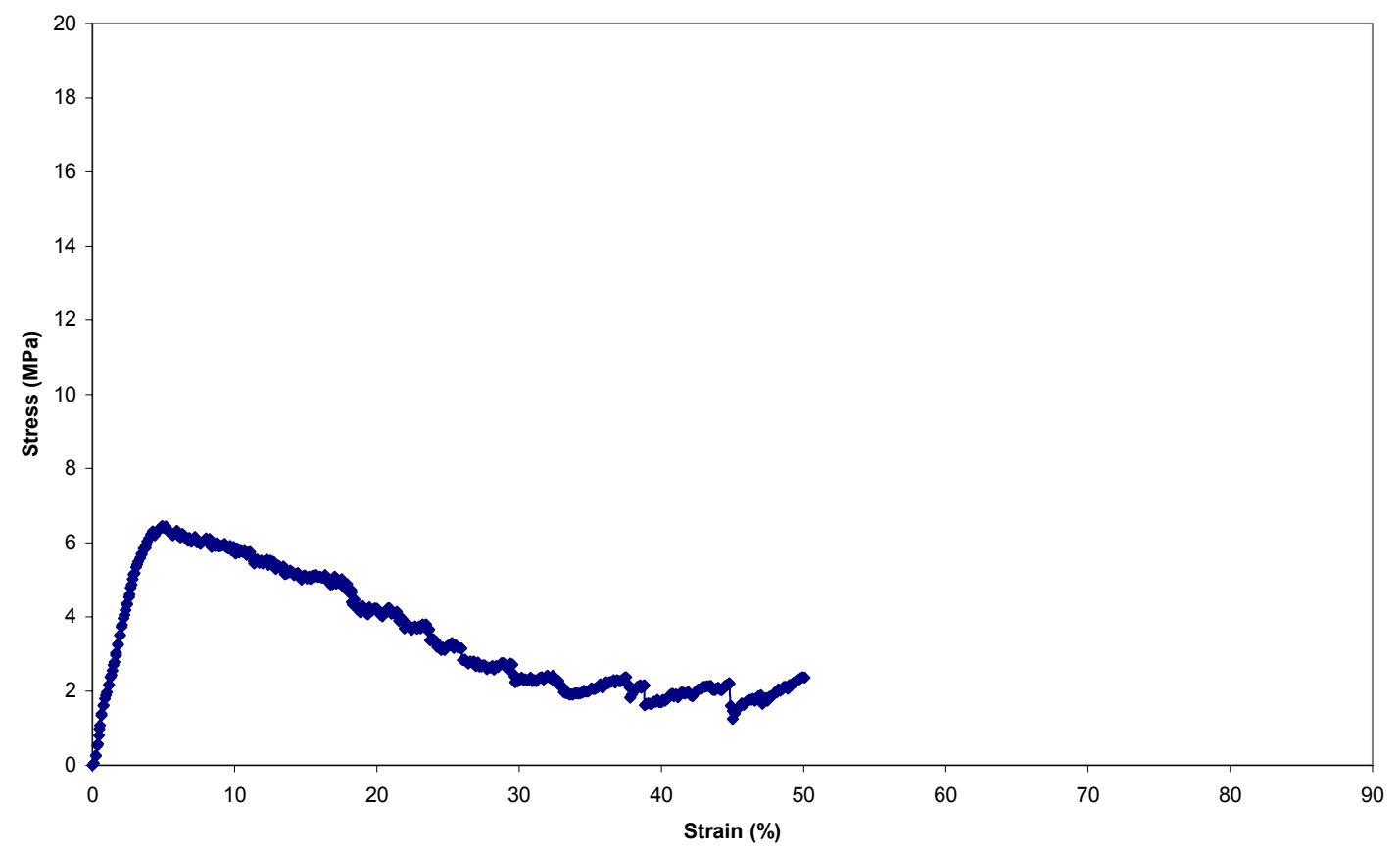

Figure A.30. Stress-Strain Plots for Samples 9 and 10 of Test 10.1 . 
10.2.1

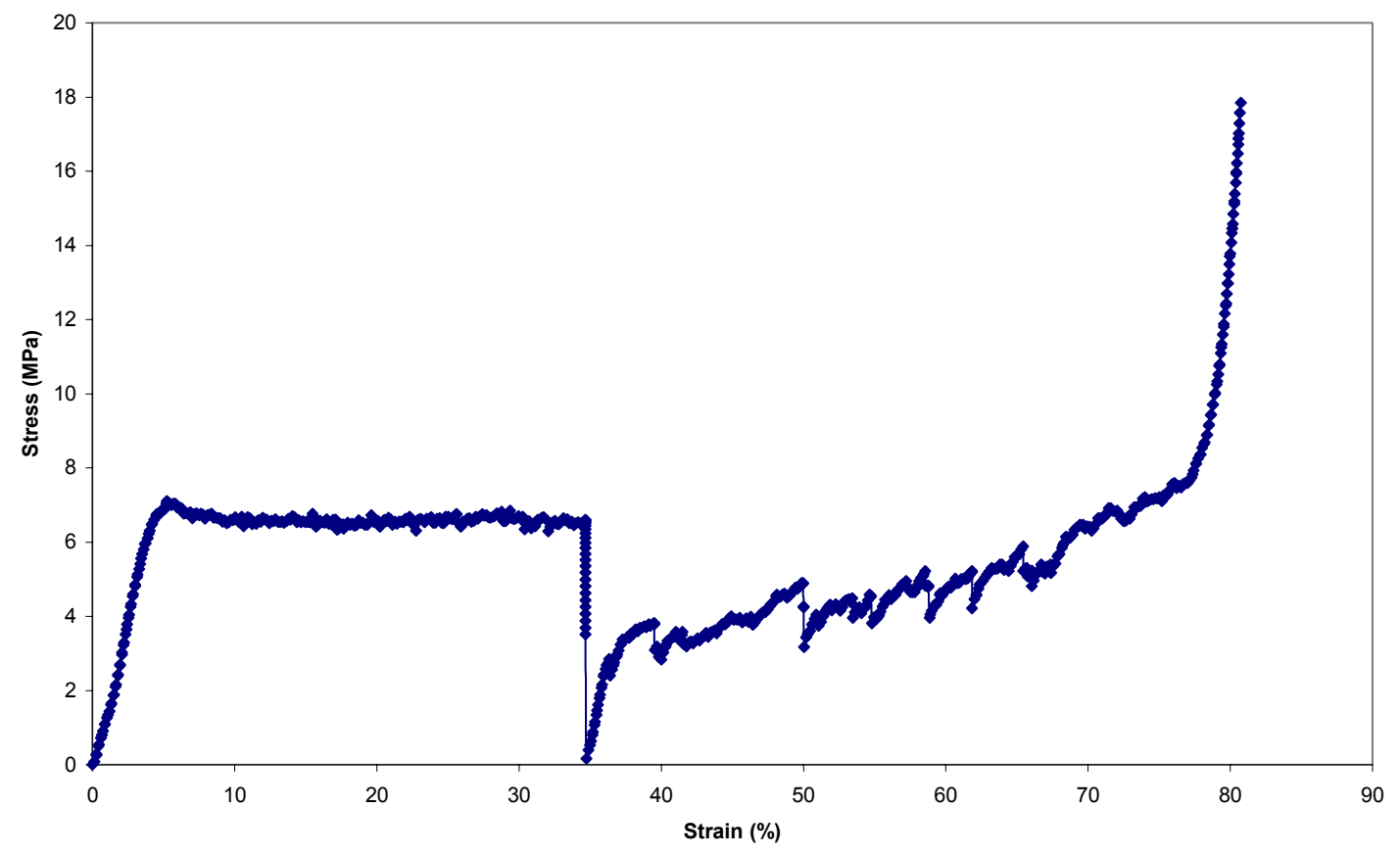

10.2.2

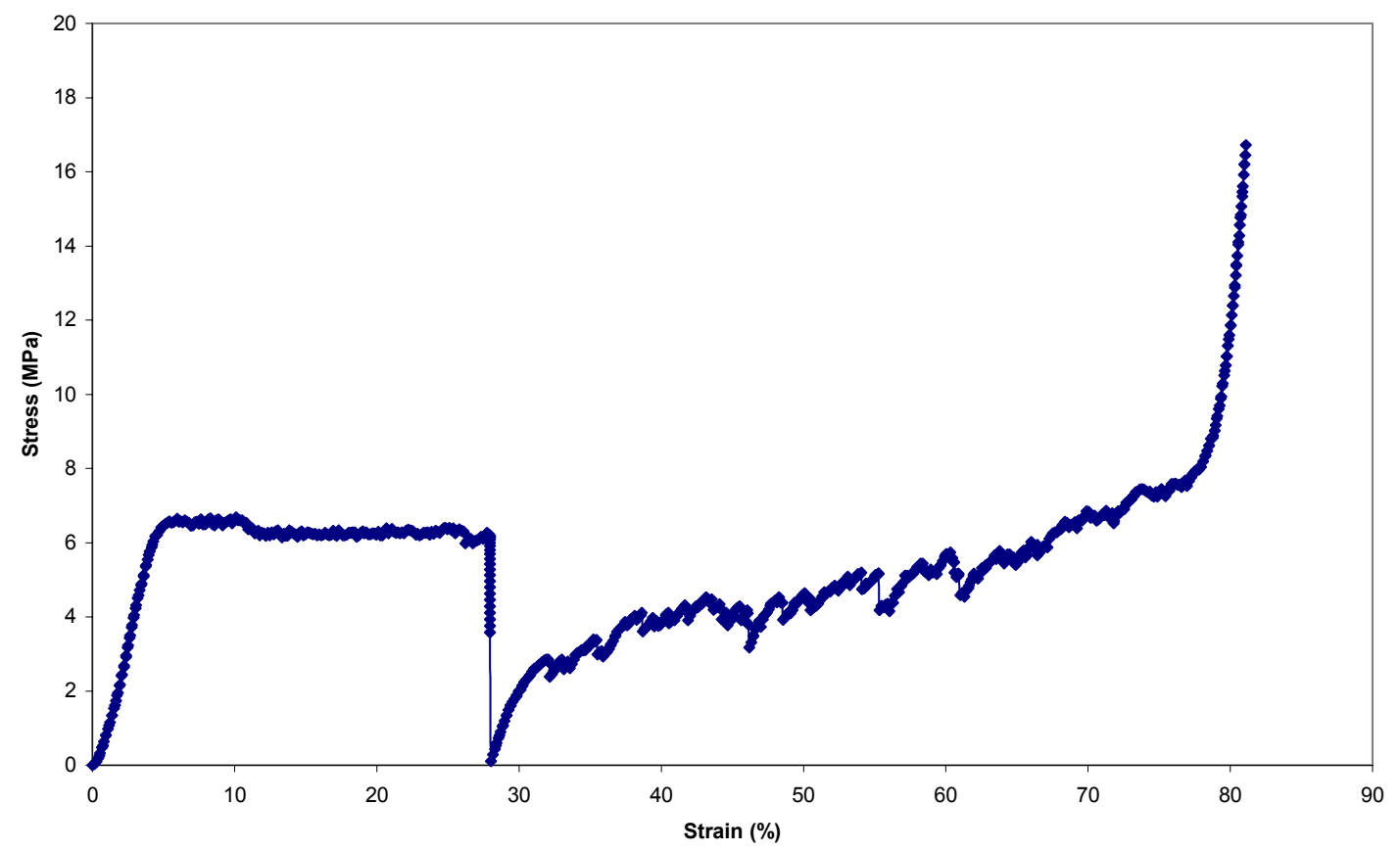

Figure A.31. Stress-Strain Plots for Samples 1 and 2 of Test 10.2. 


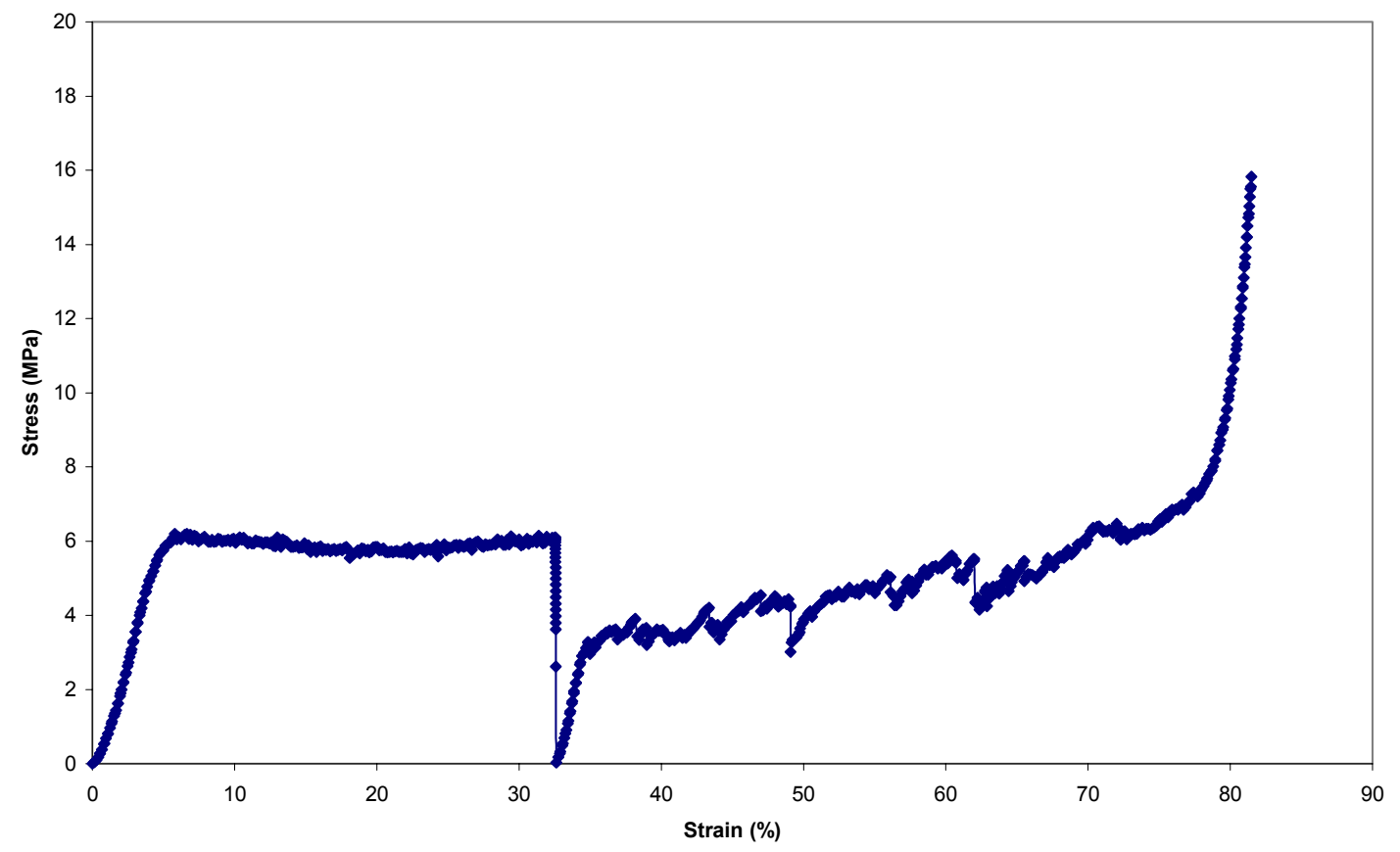

10.2.4

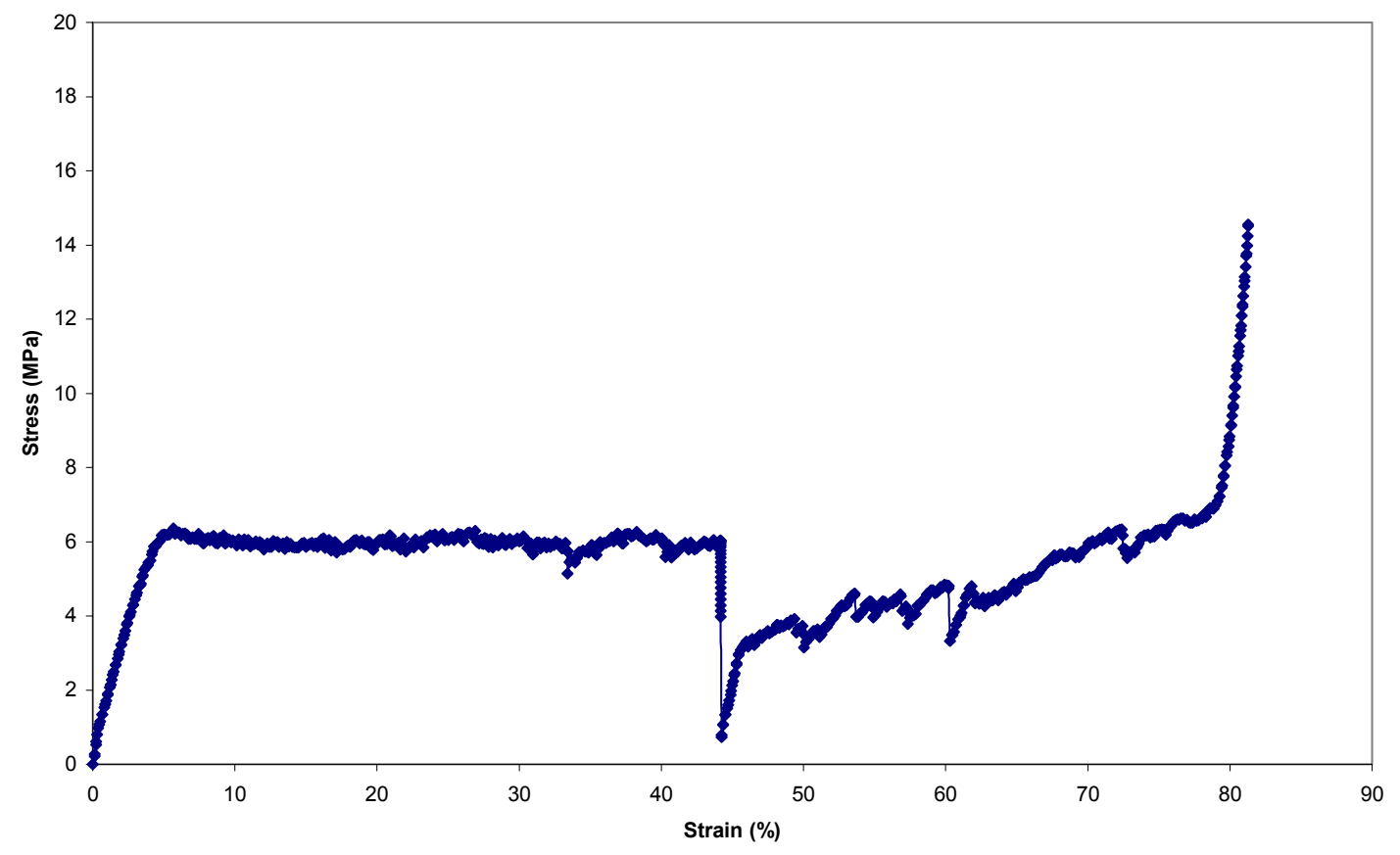

Figure A.32. Stress-Strain Plots for Samples 3 and 4 of Test 10.2. 


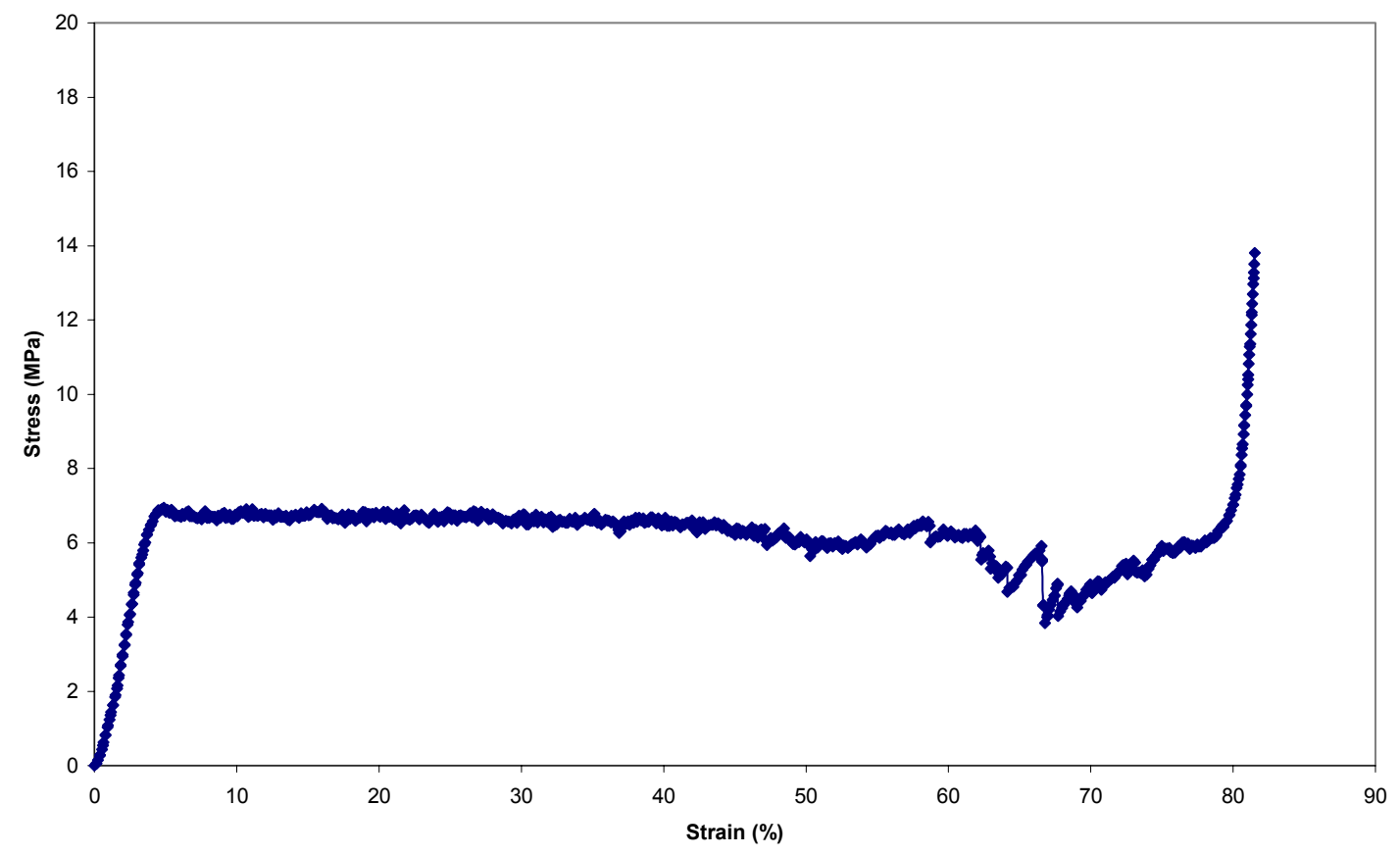

10.2.6

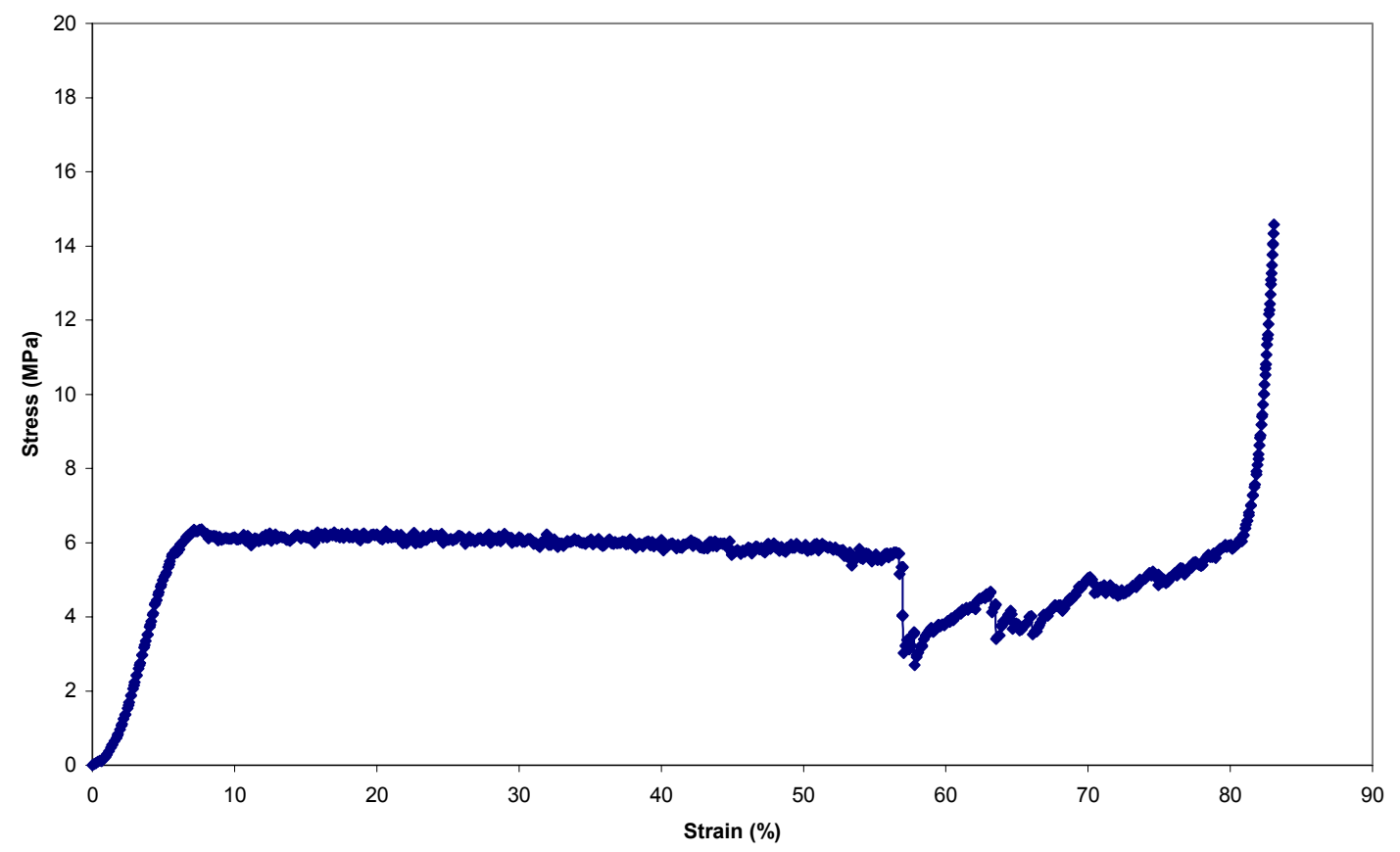

Figure A.33. Stress-Strain Plots for Samples 5 and 6 of Test 10.2. 


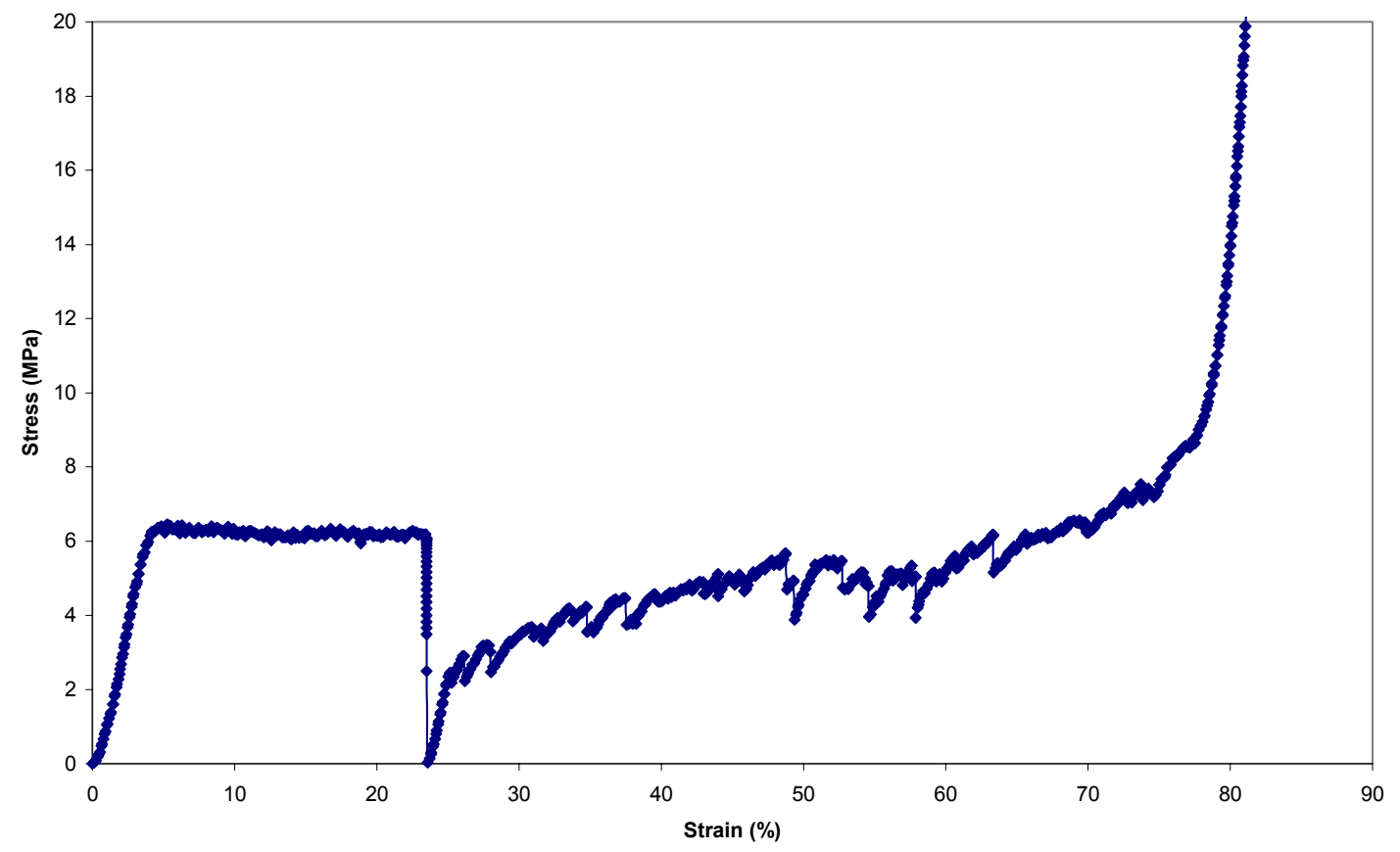

10.2 .8

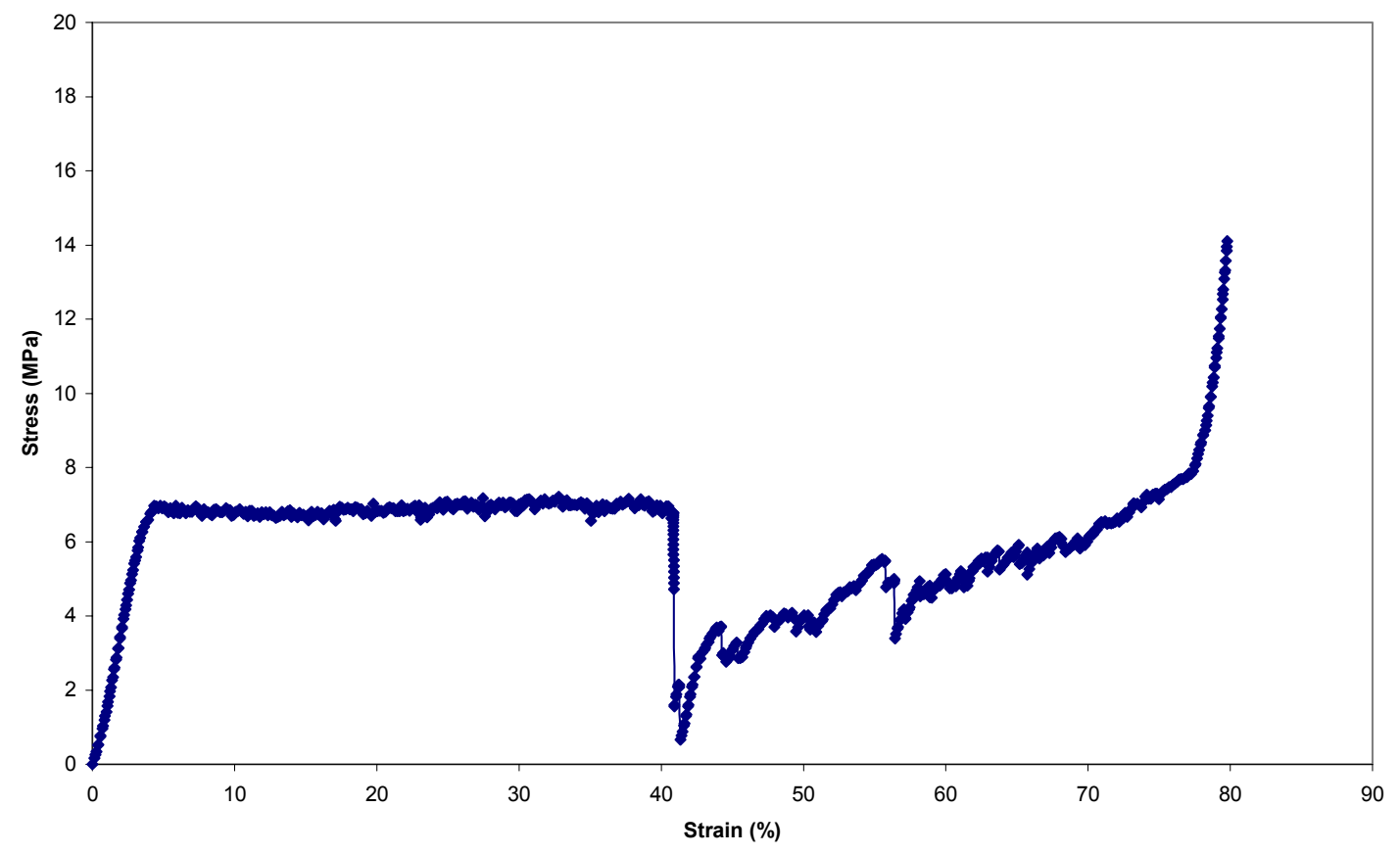

Figure A.34. Stress-Strain Plots for Samples 7 and 8 of Test 10.2. 


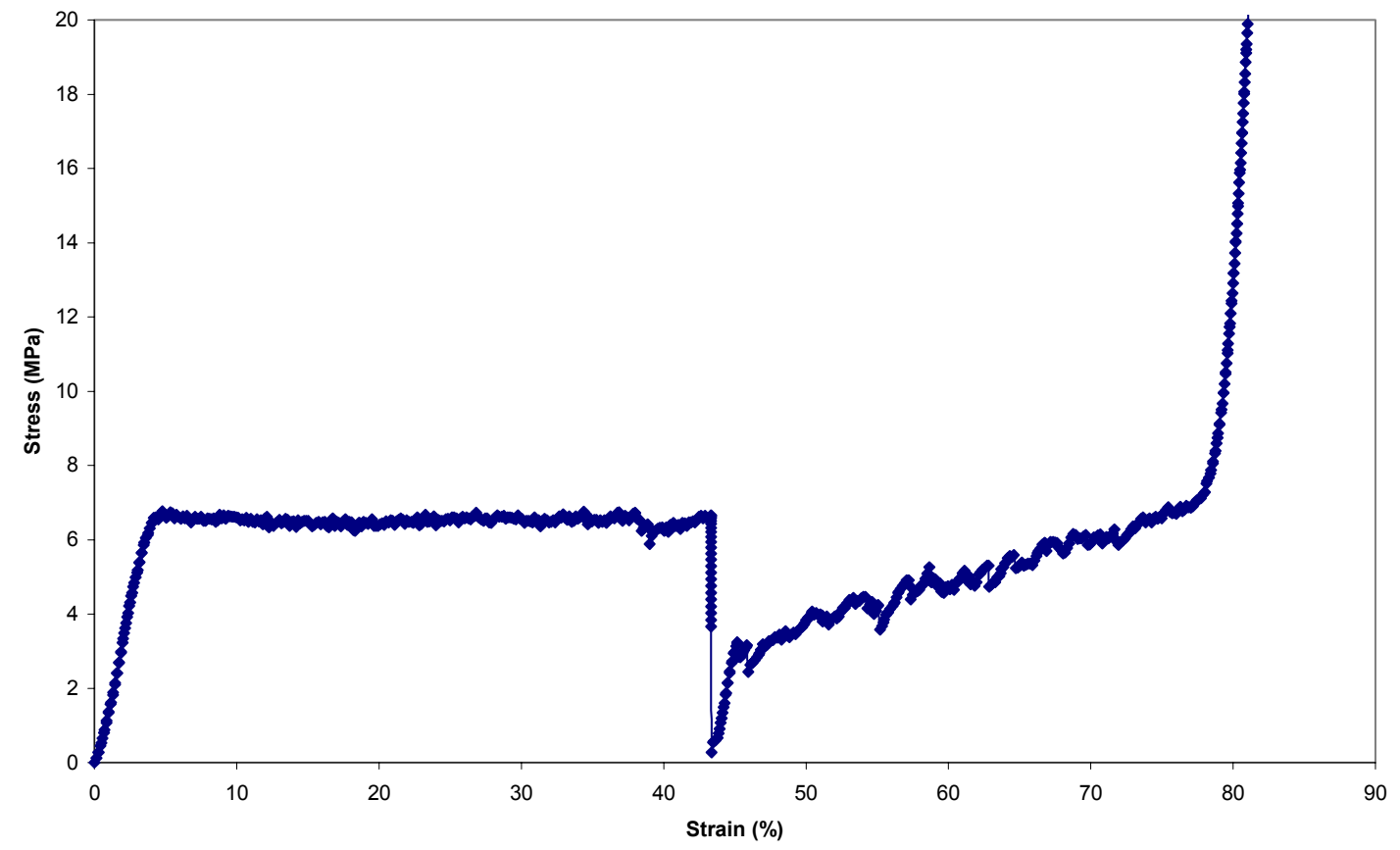

Figure A.35. Stress-Strain Plot for Sample 9 of Test 10.2. 


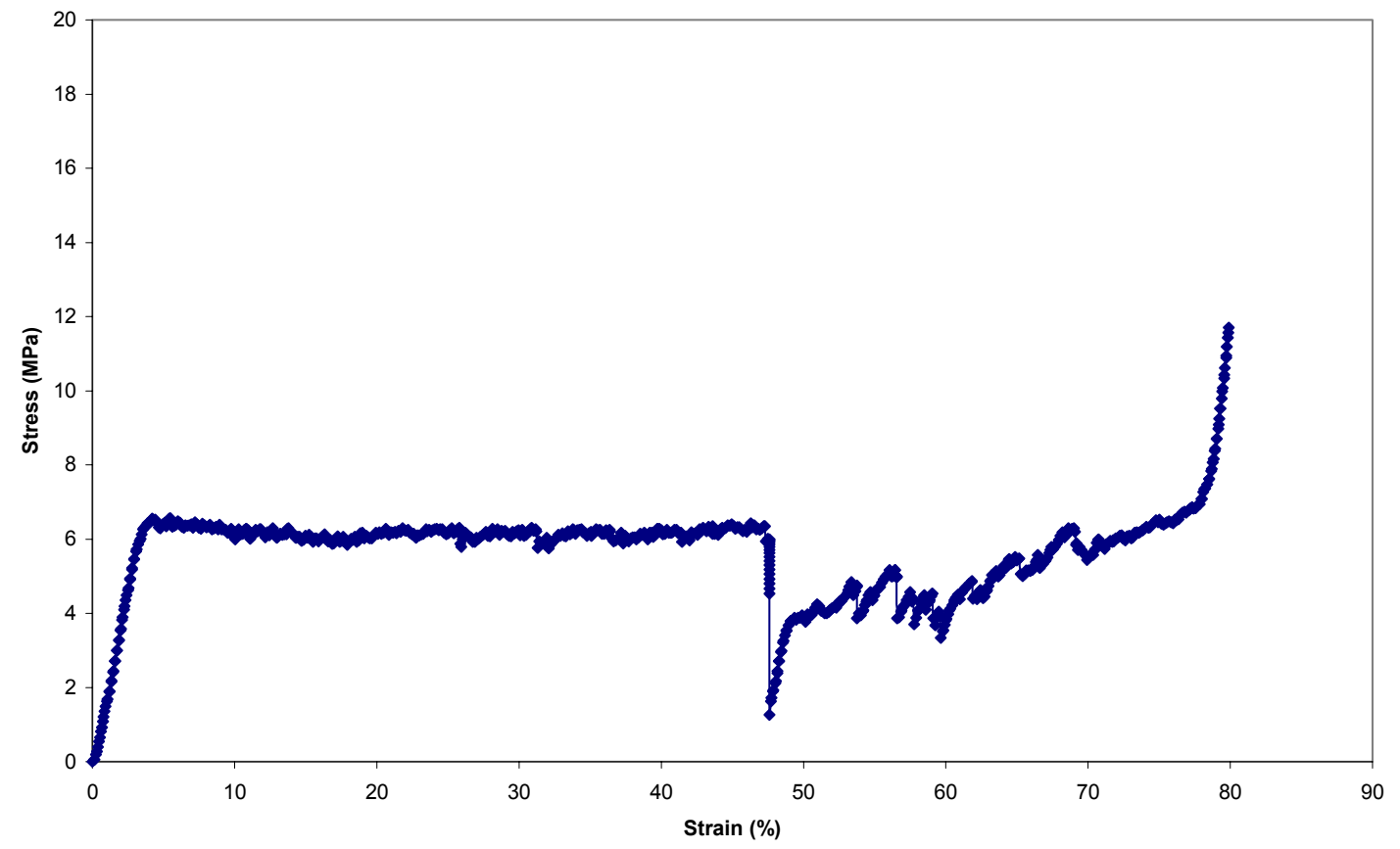

10.3.2

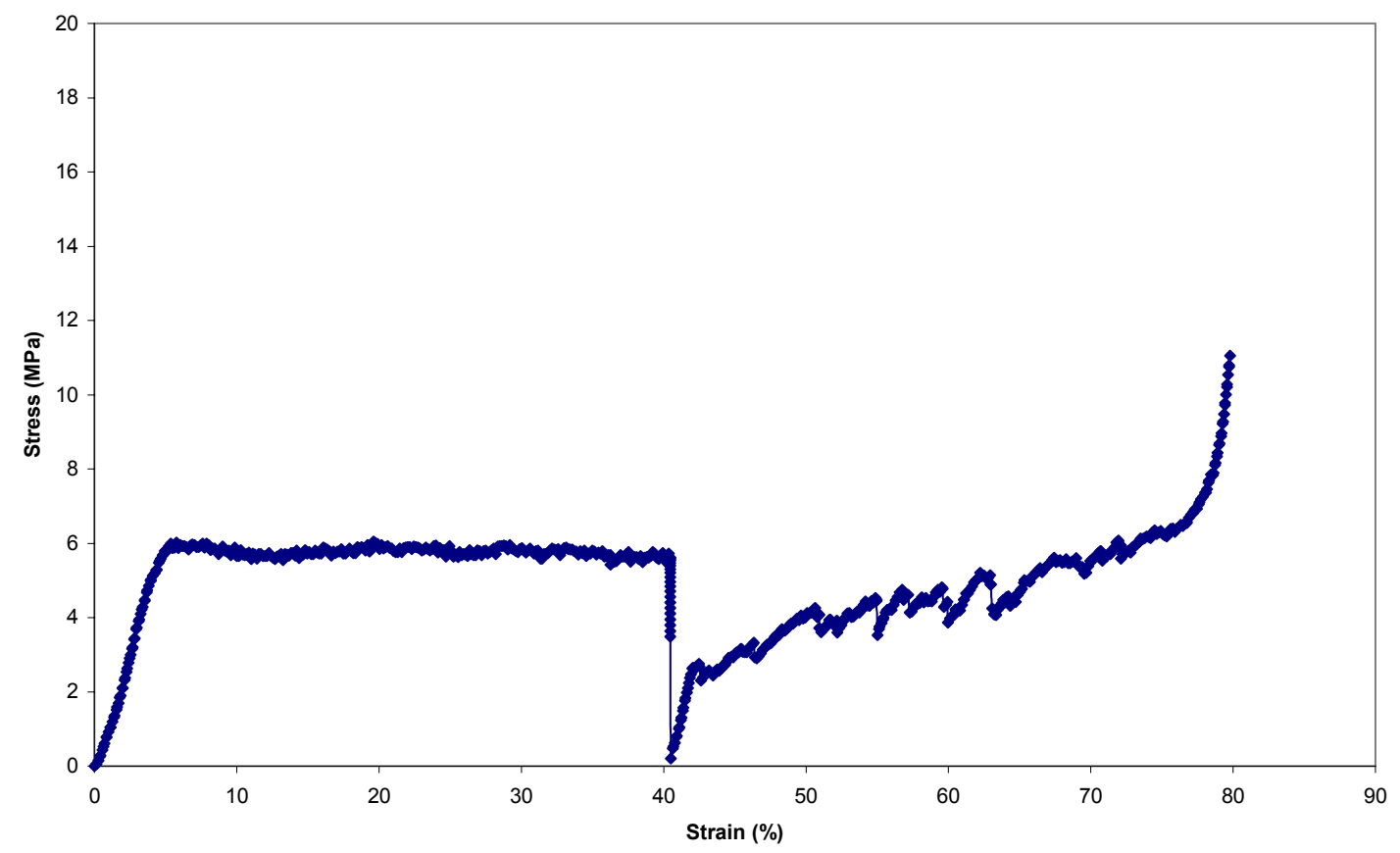

Figure A.36. Stress-Strain Plots for Samples 1 and 2 of Test 10.3. 


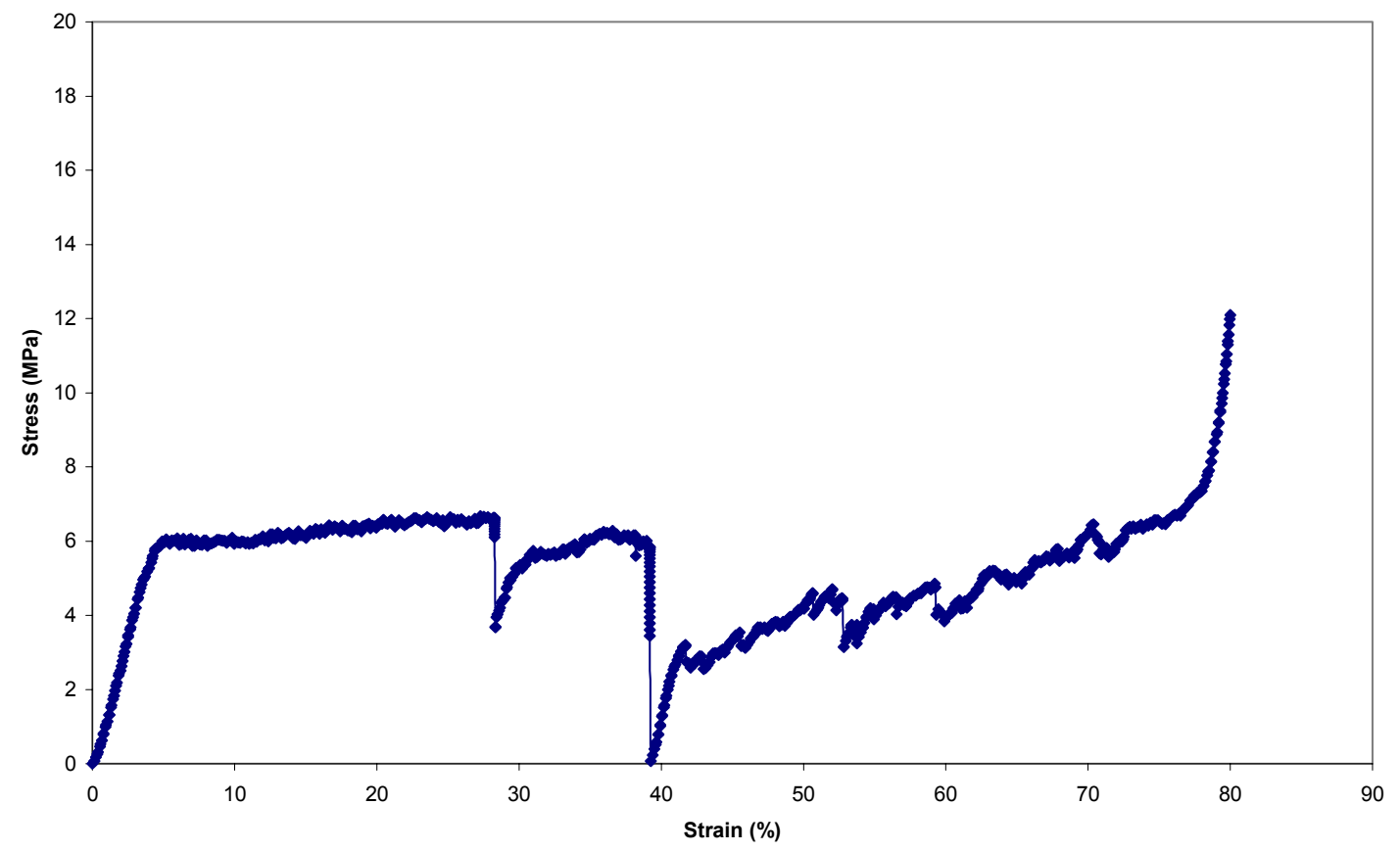

10.3.4

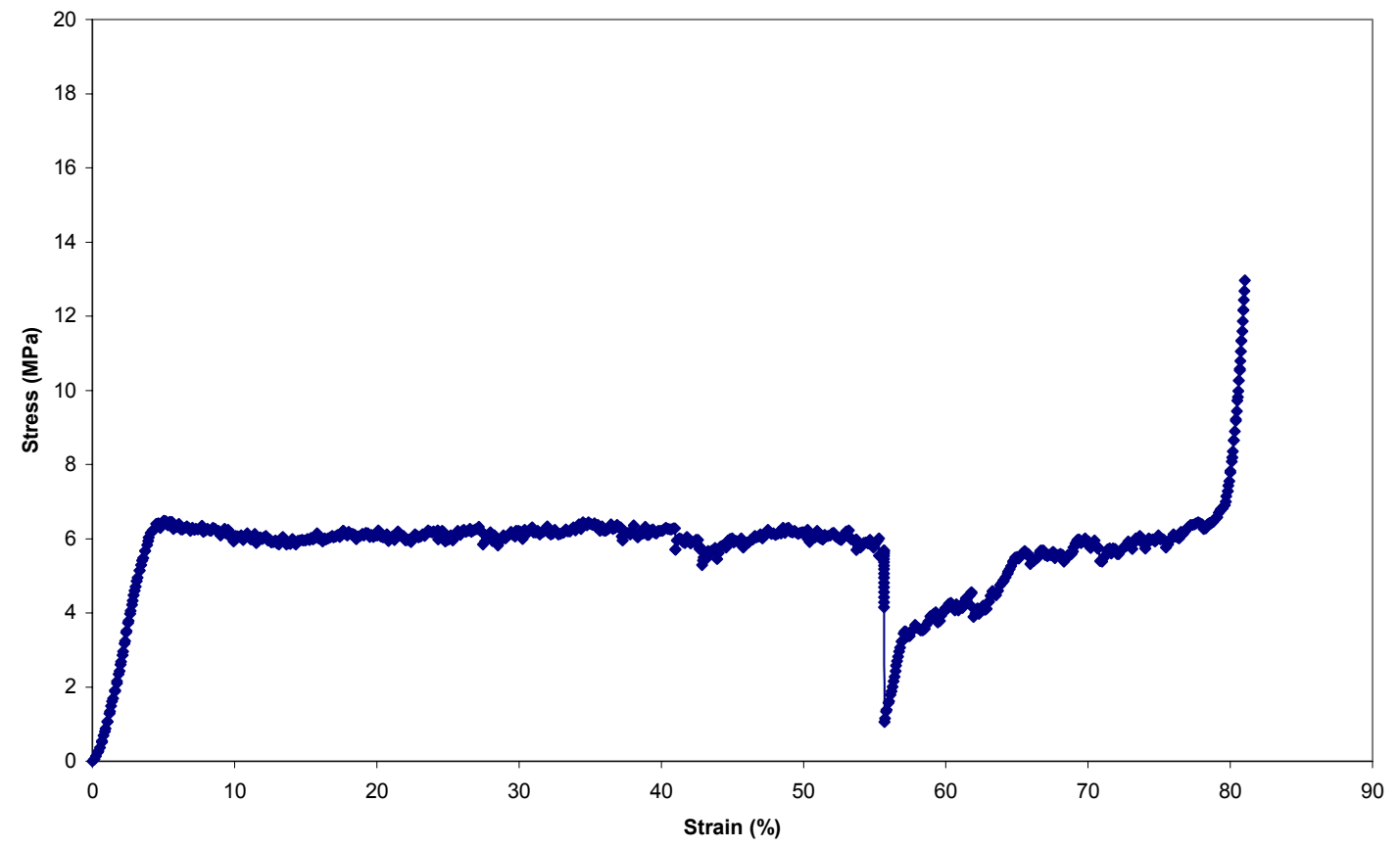

Figure A.37. Stress-Strain Plots for Samples 3 and 4 of Test 10.3. 


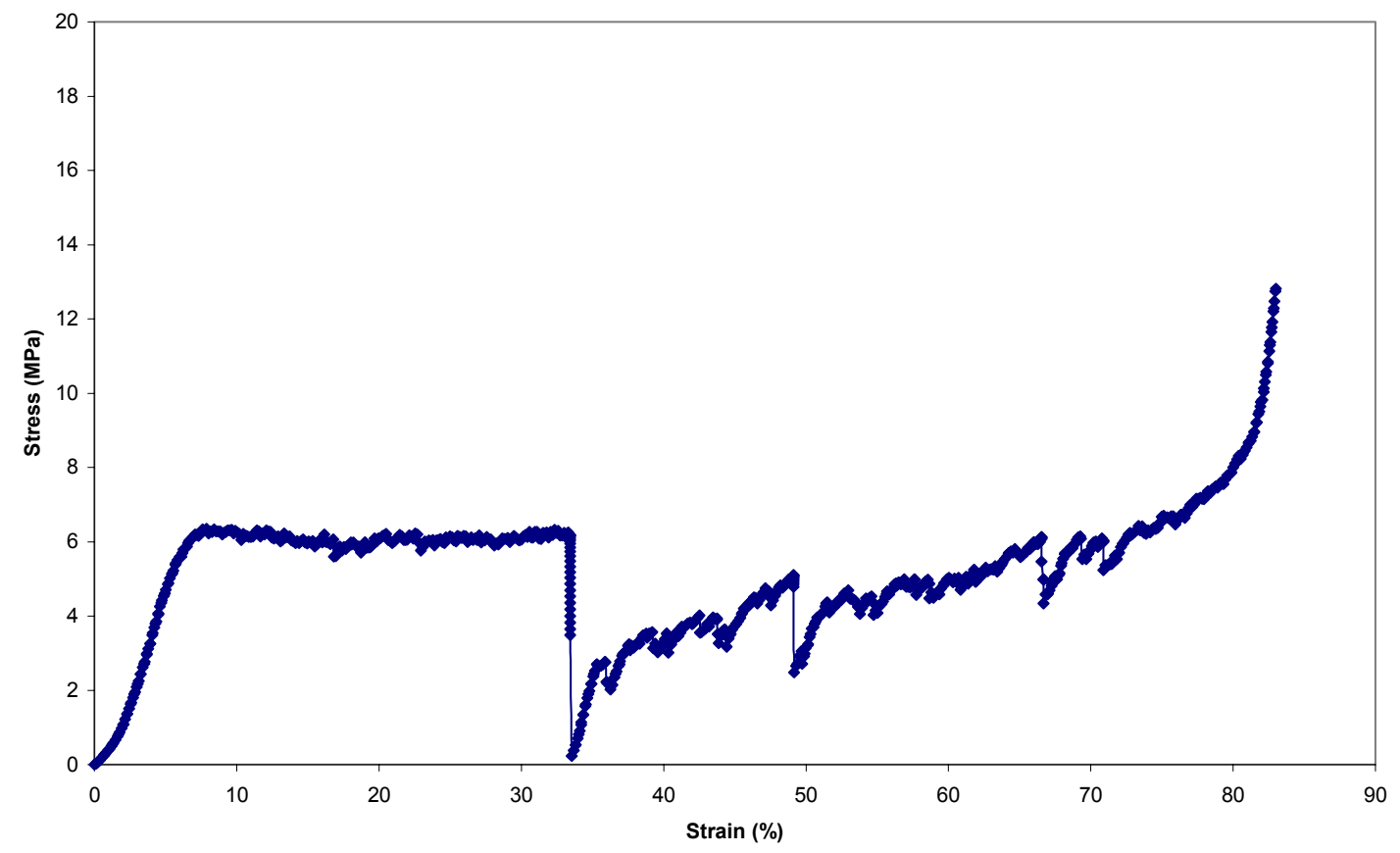

10.3.6

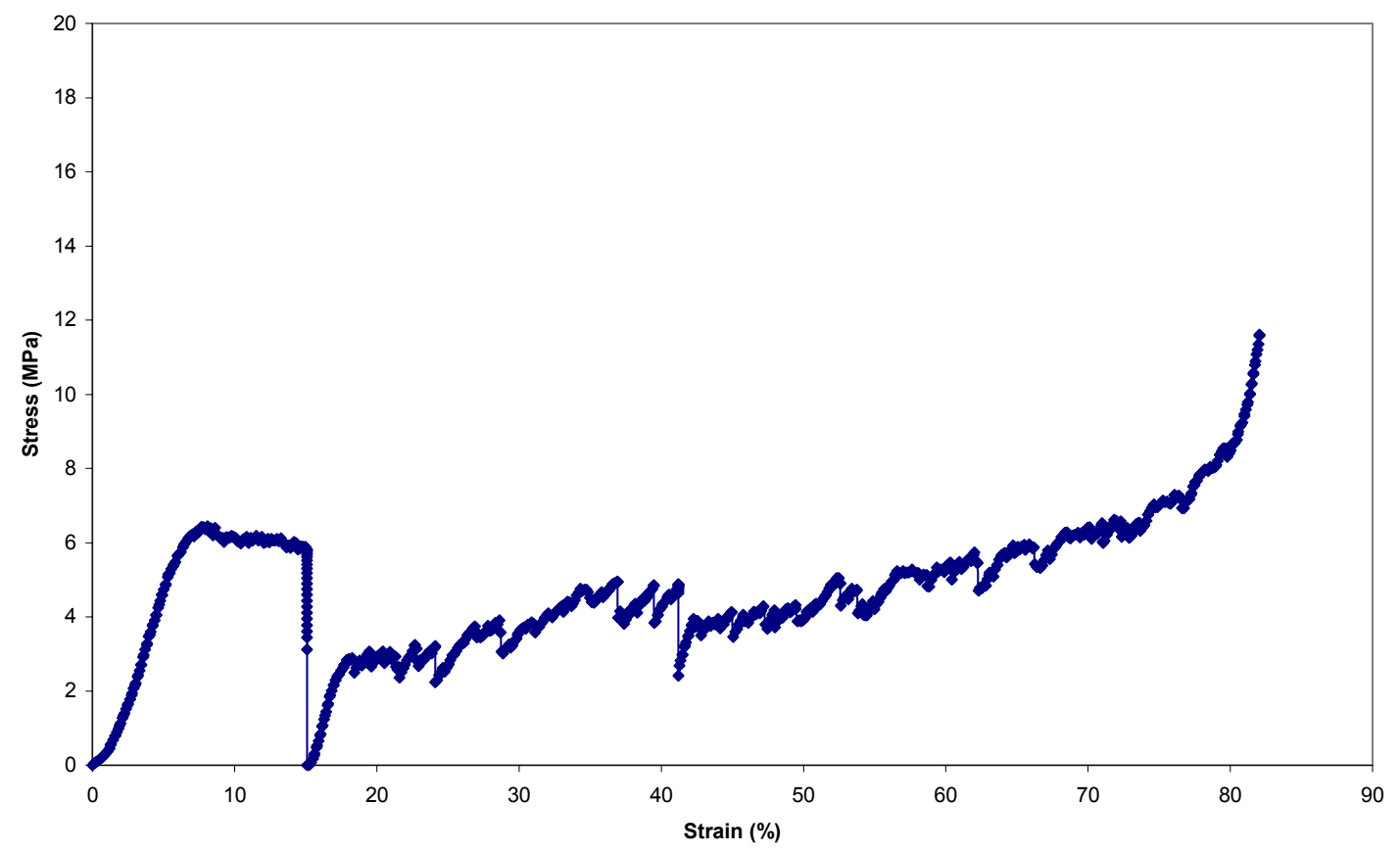

Figure A.38. Stress-Strain Plots for Samples 5 and 6 of Test 10.3. 


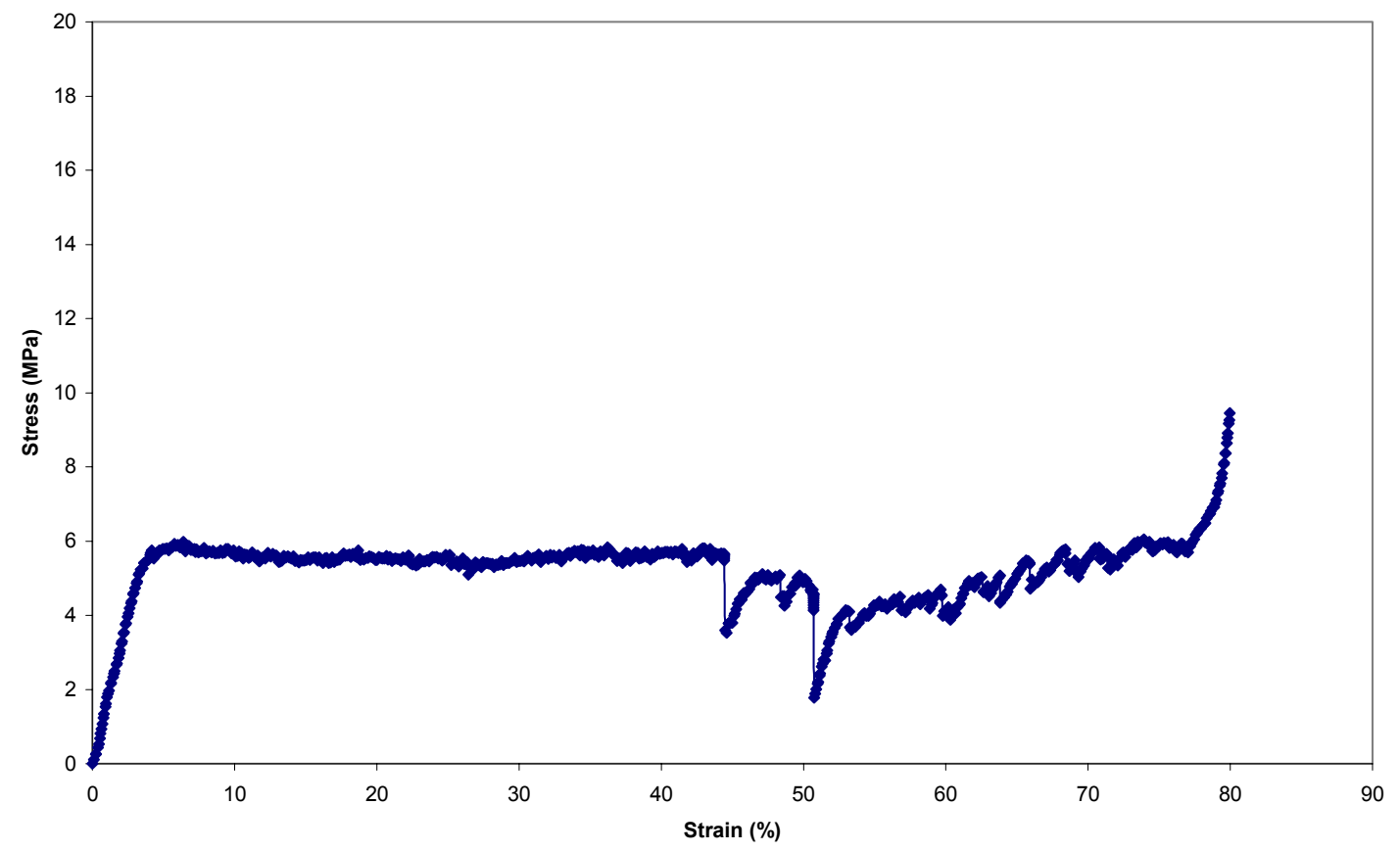

10.3.8

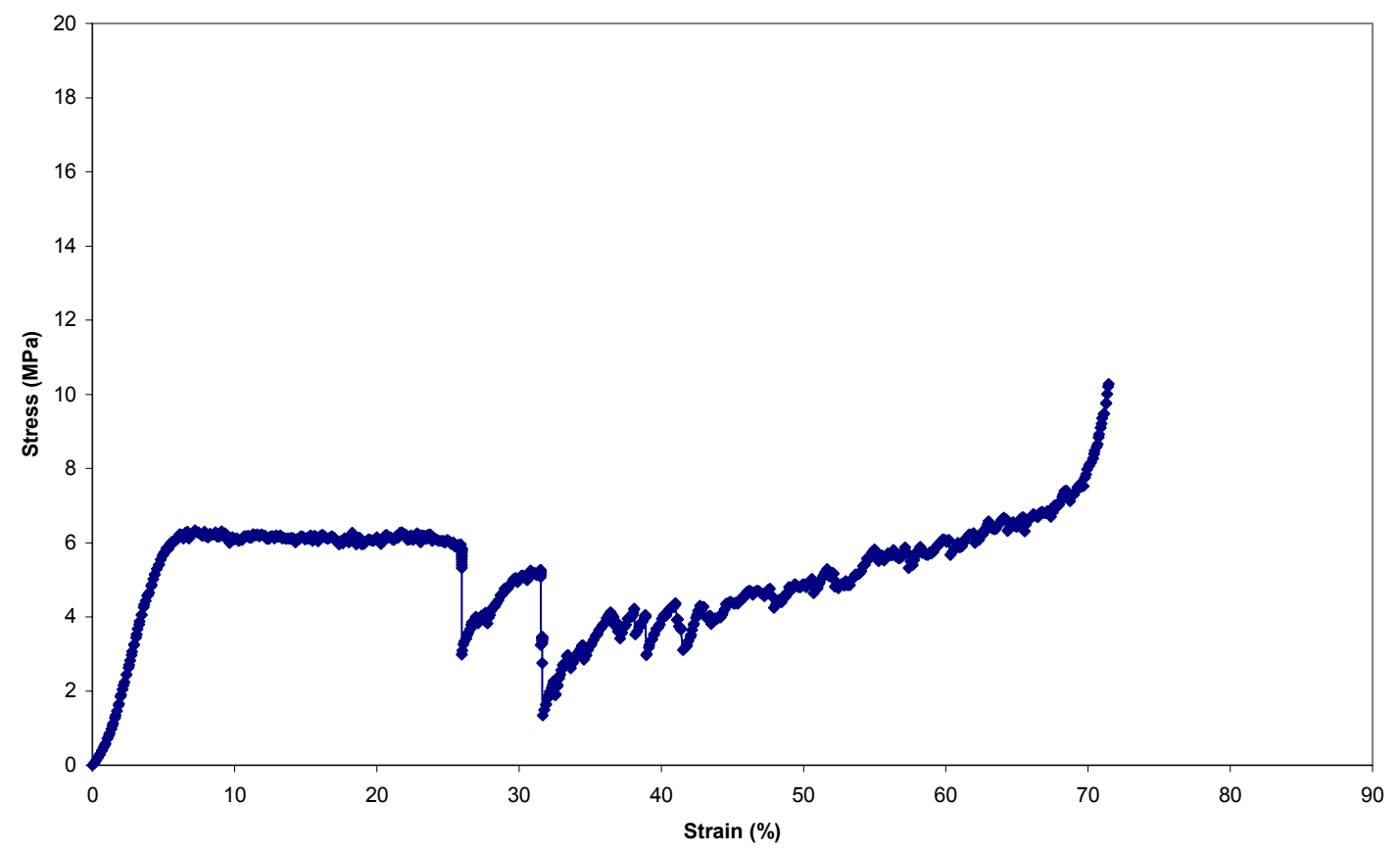

Figure A.39. Stress-Strain Plots for Samples 7 and 8 of Test 10.3. 


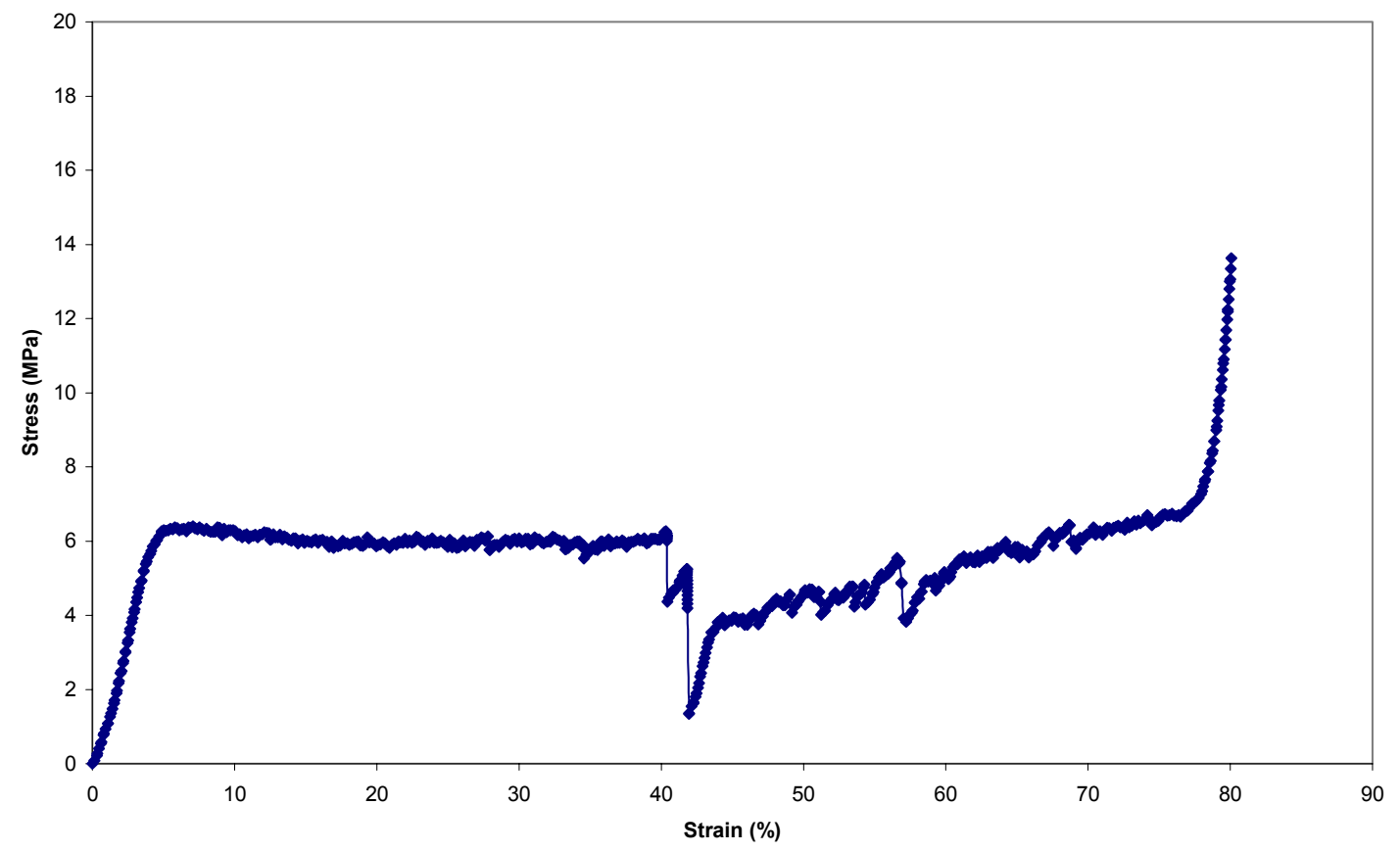

10.3.10

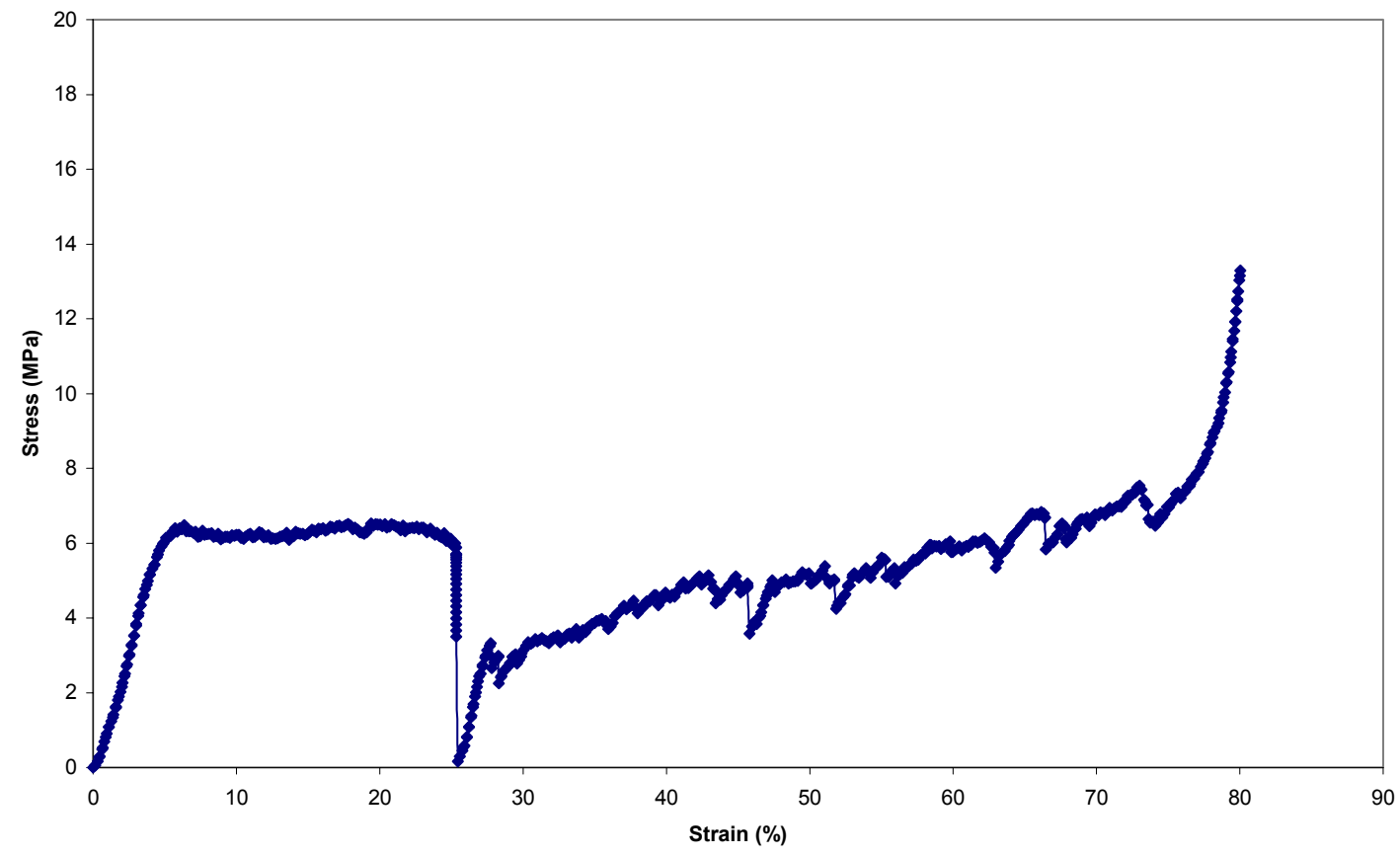

Figure A.40. Stress-Strain Plots for Samples 9 and 10 of Test 10.3 . 


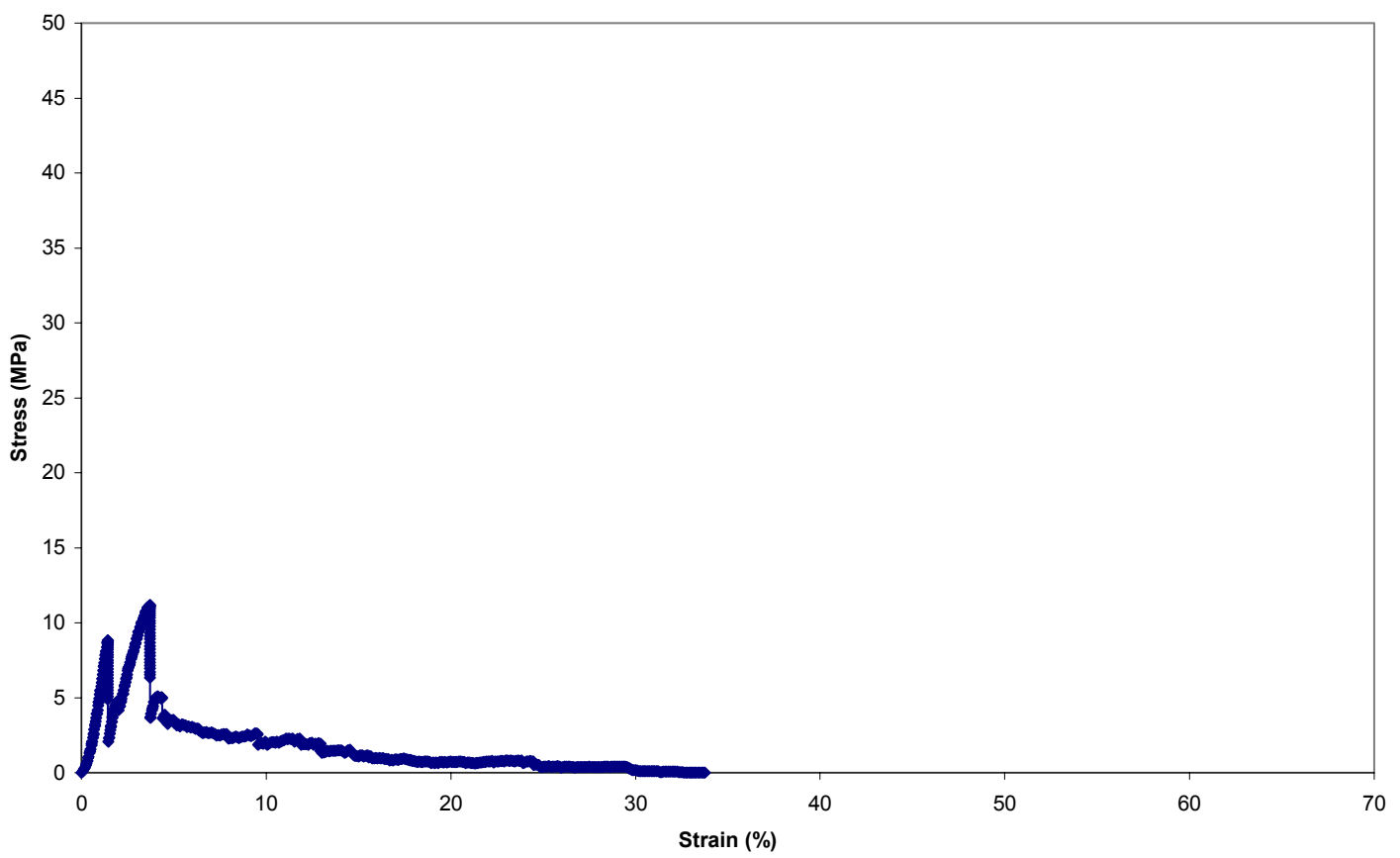

20.1.2

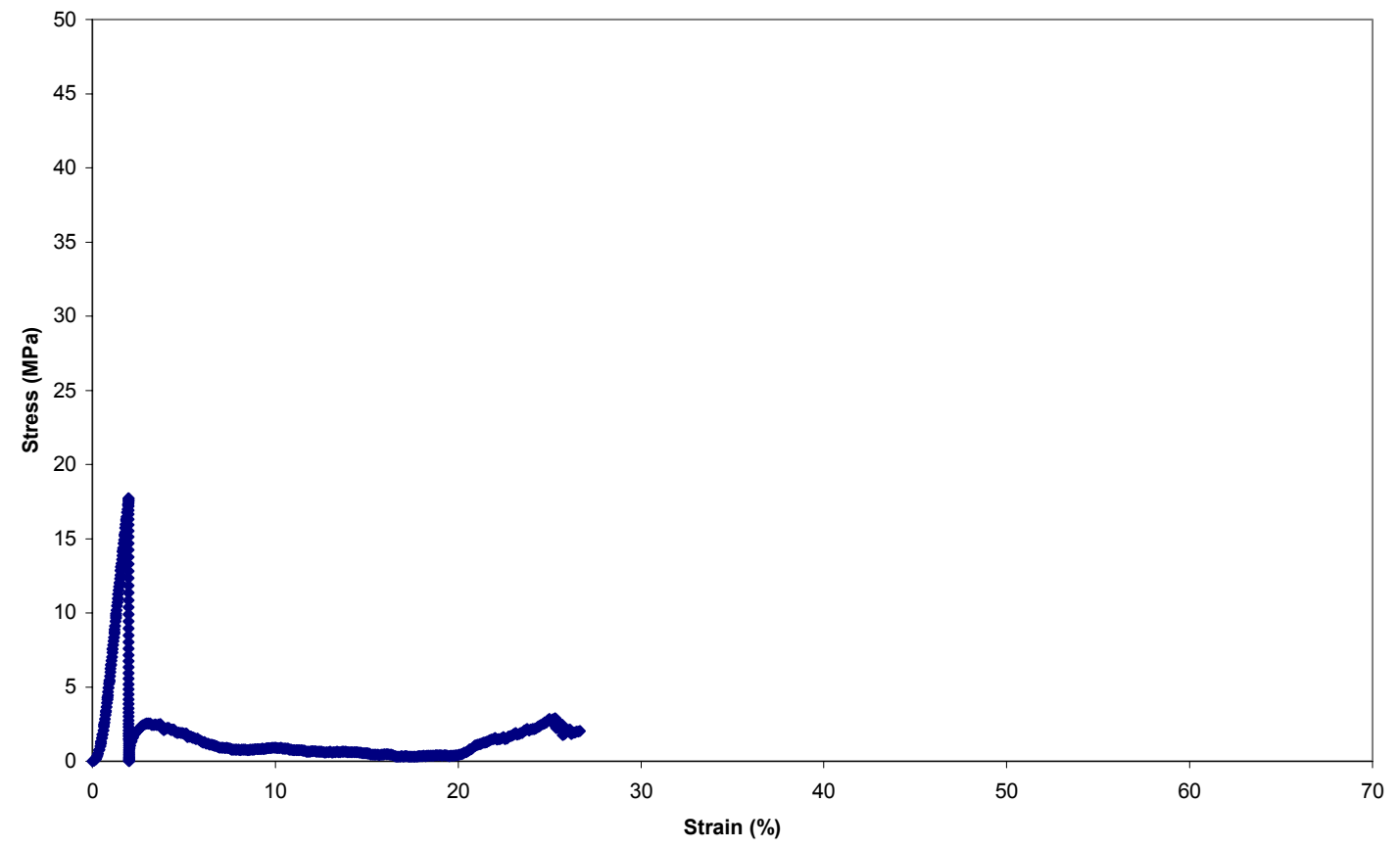

Figure A.41. Stress-Strain Plots for Samples 1 and 2 of Test 20.1. 


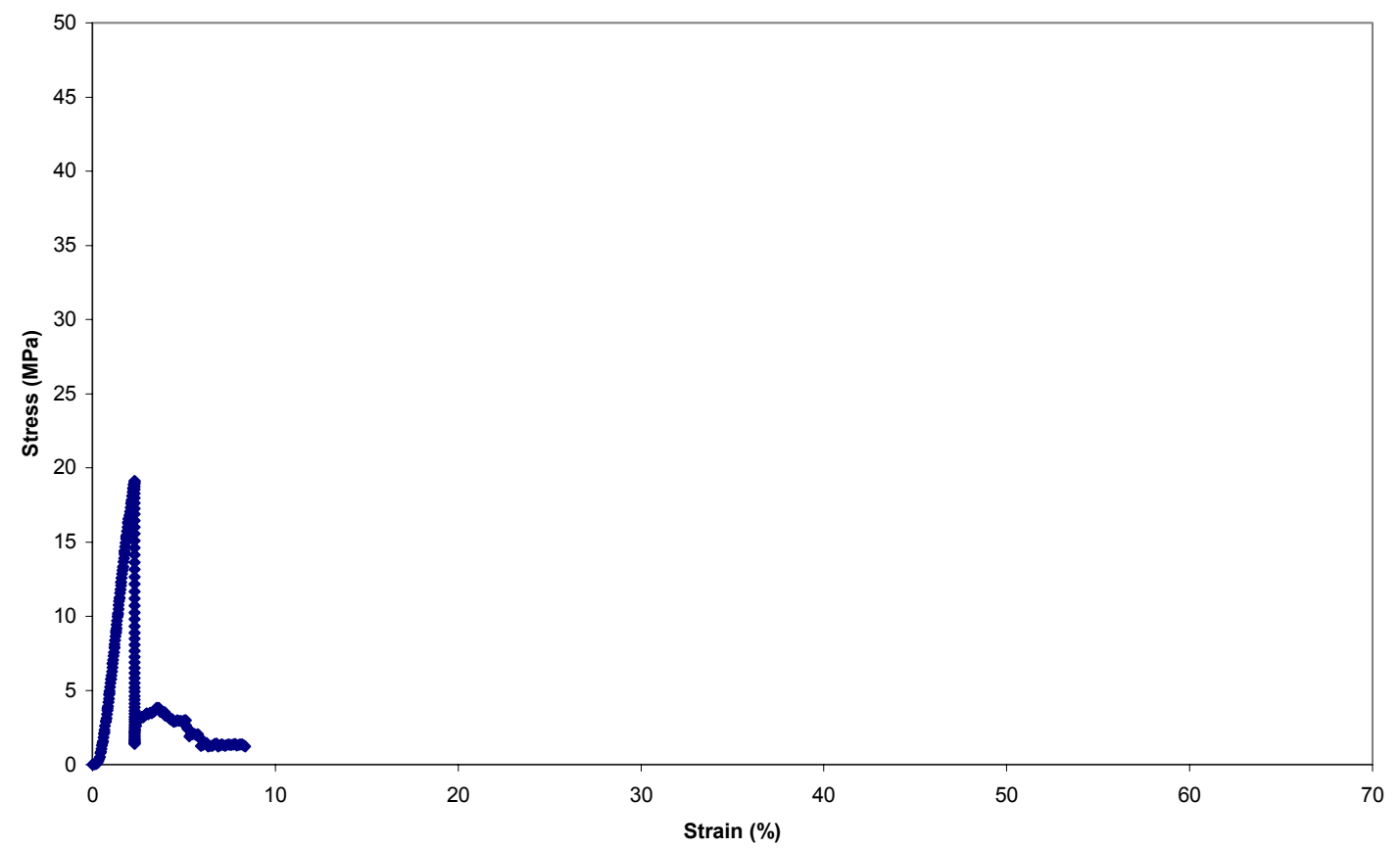

20.1.4

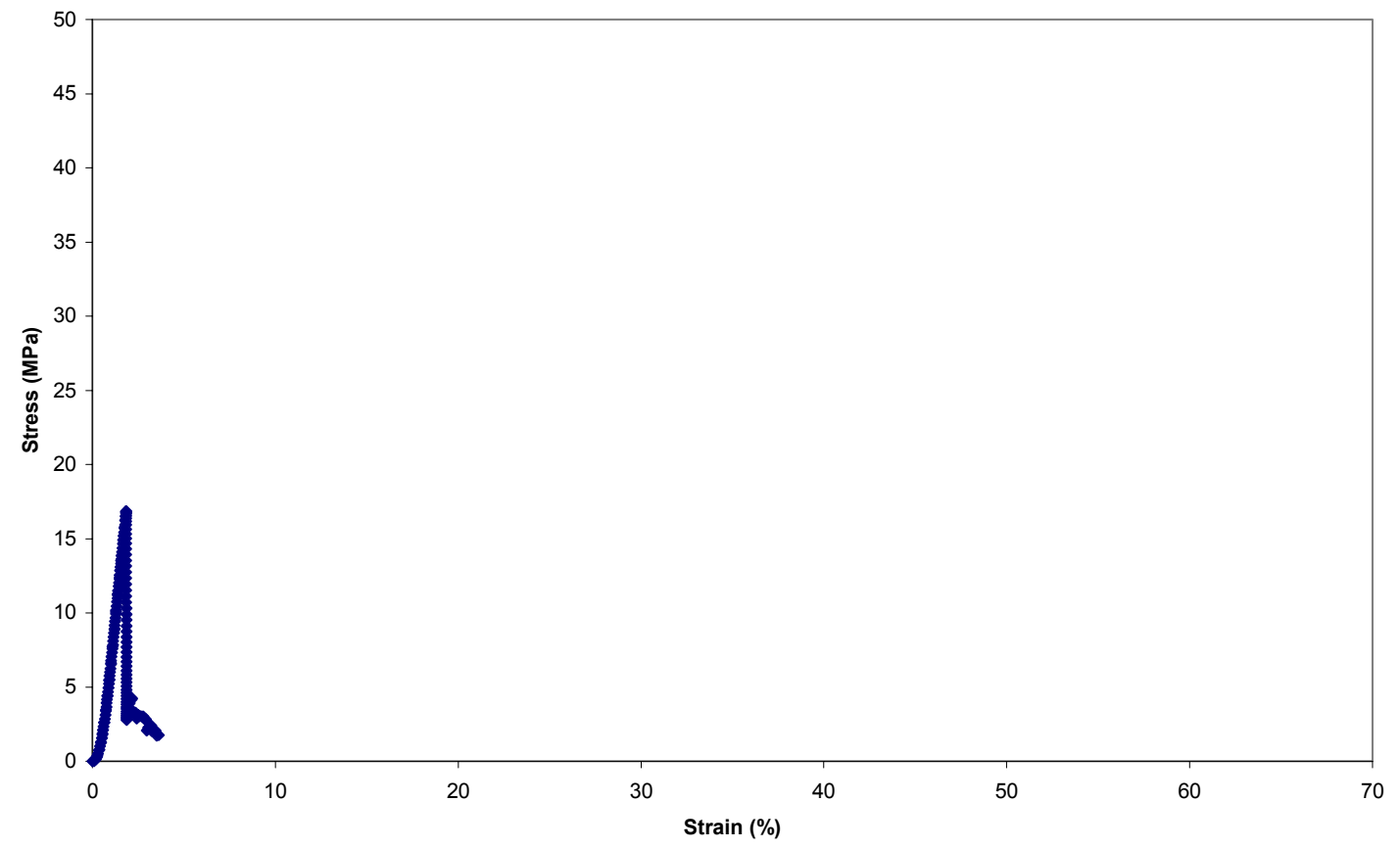

Figure A.42. Stress-Strain Plots for Samples 3 and 4 of Test 20.1. 


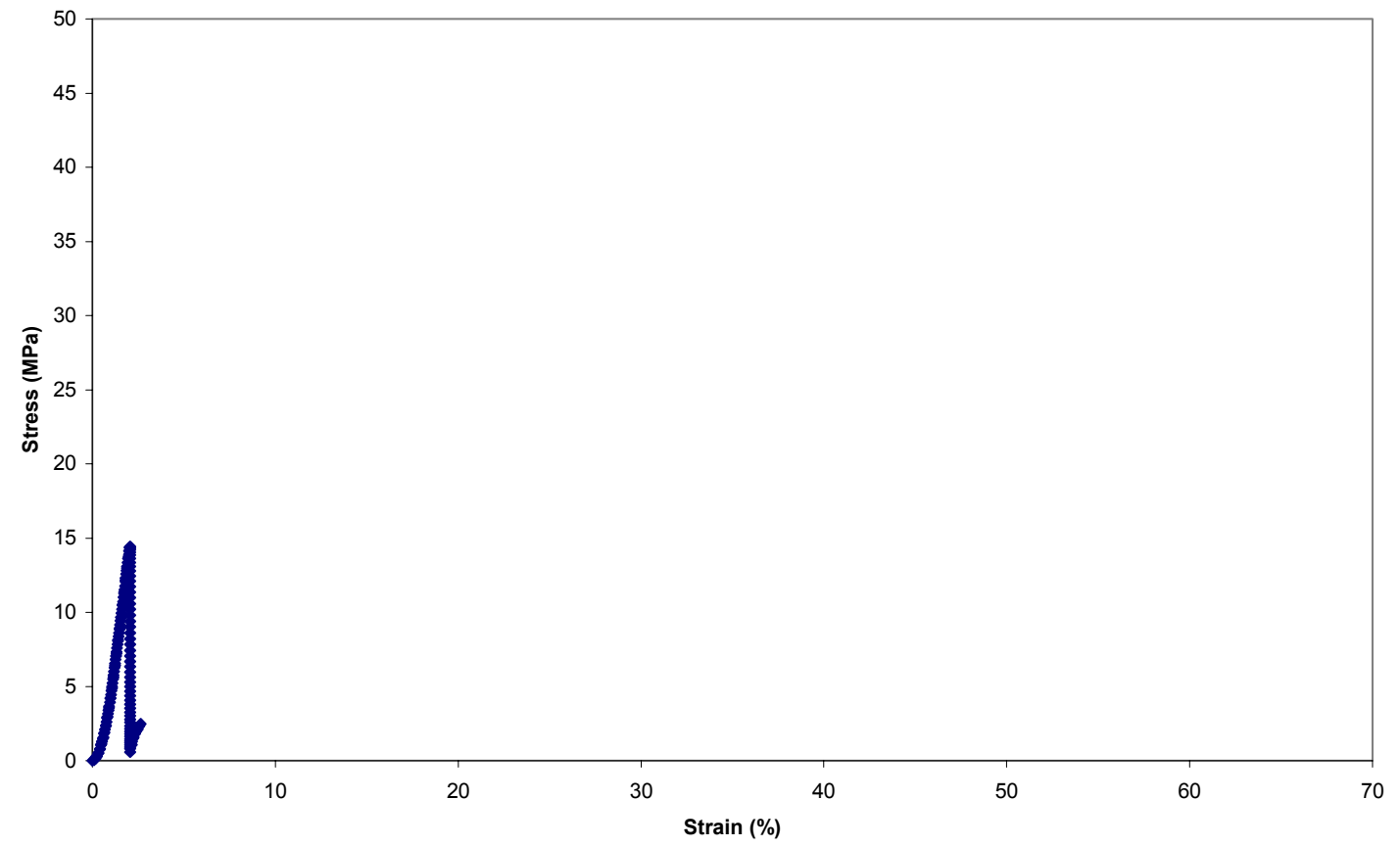

20.1.6

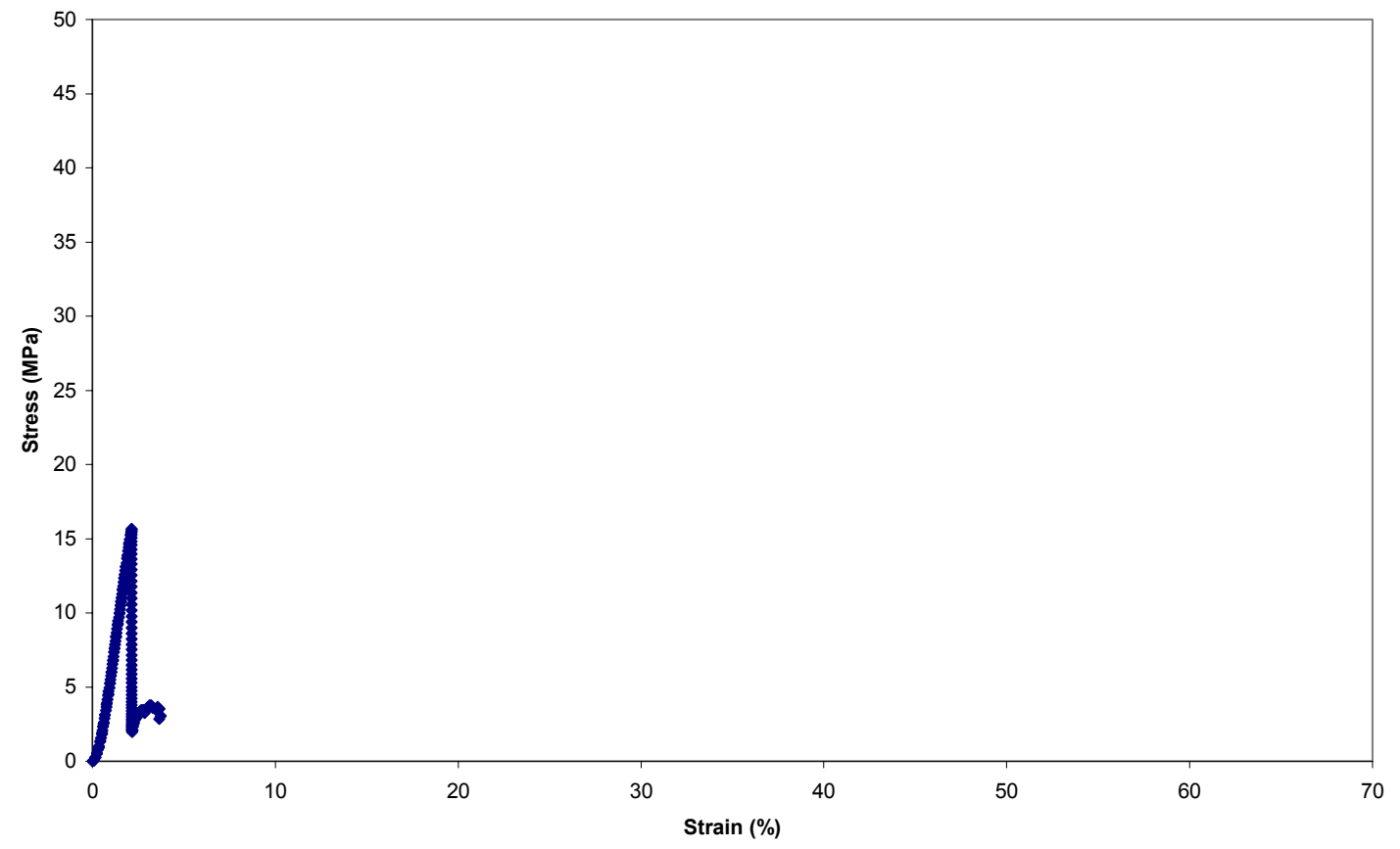

Figure A.43. Stress-Strain Plots for Samples 5 and 6 of Test 20.1. 


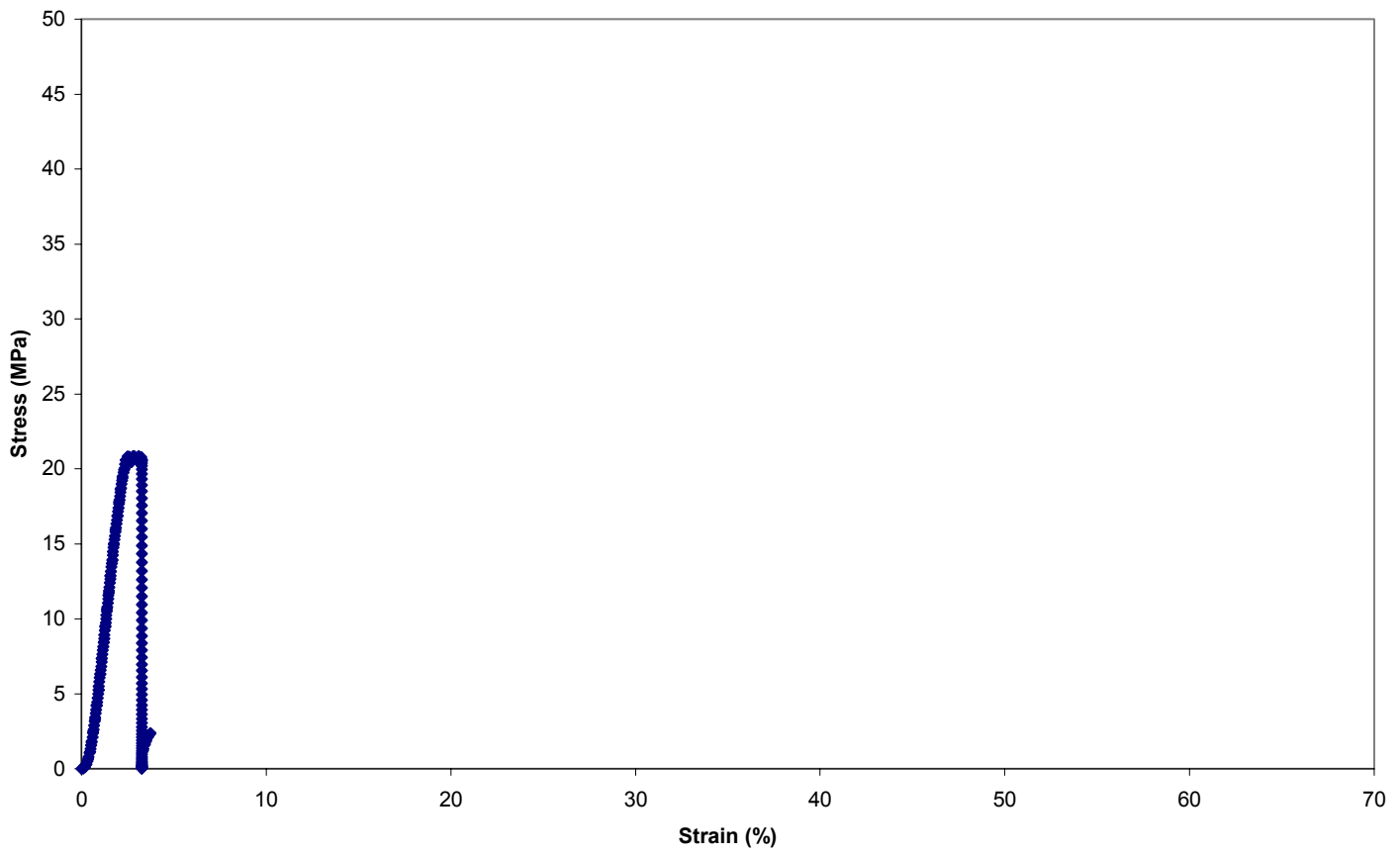

20.1.8

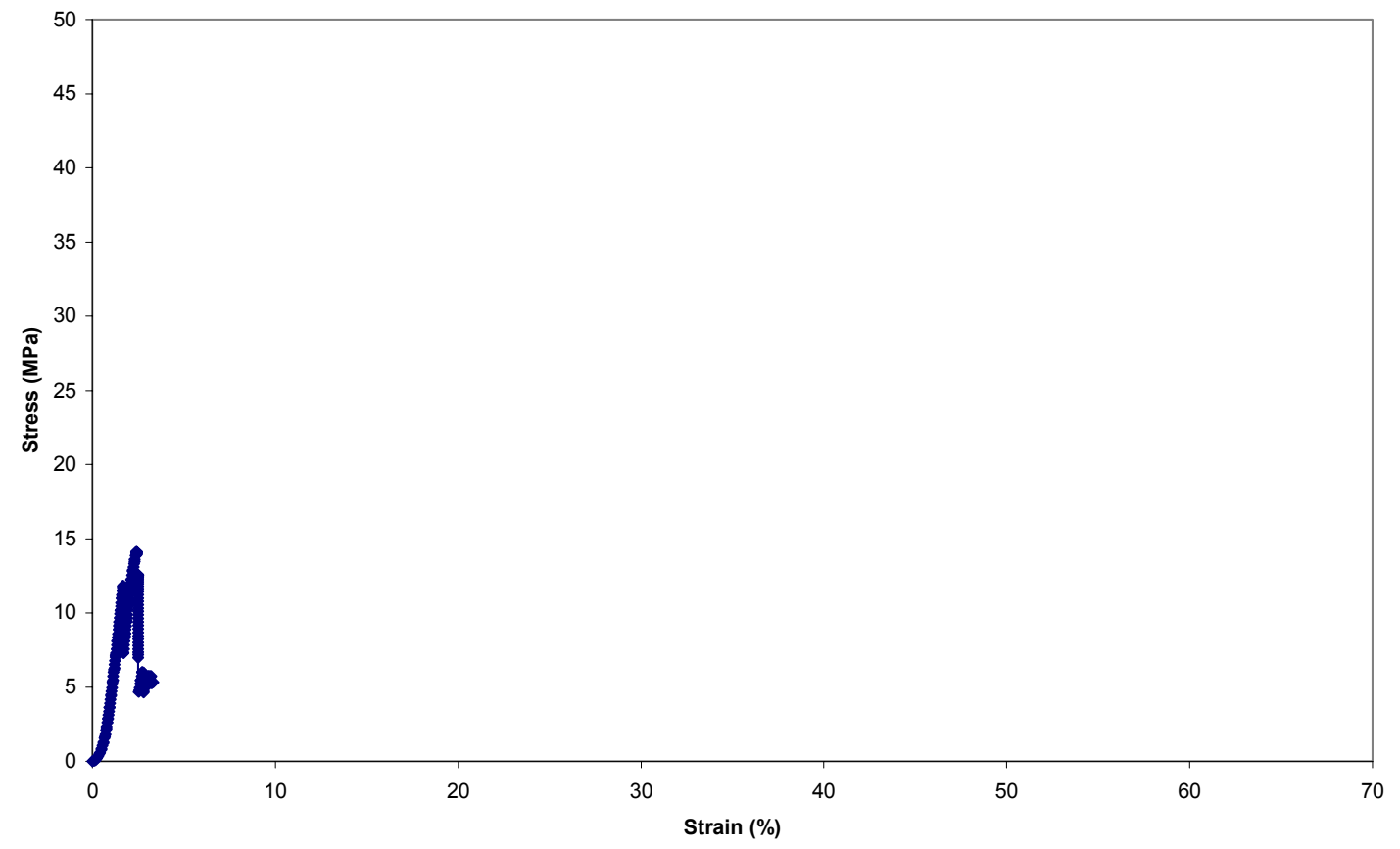

Figure A.44. Stress-Strain Plots for Samples 7 and 8 of Test 20.1. 


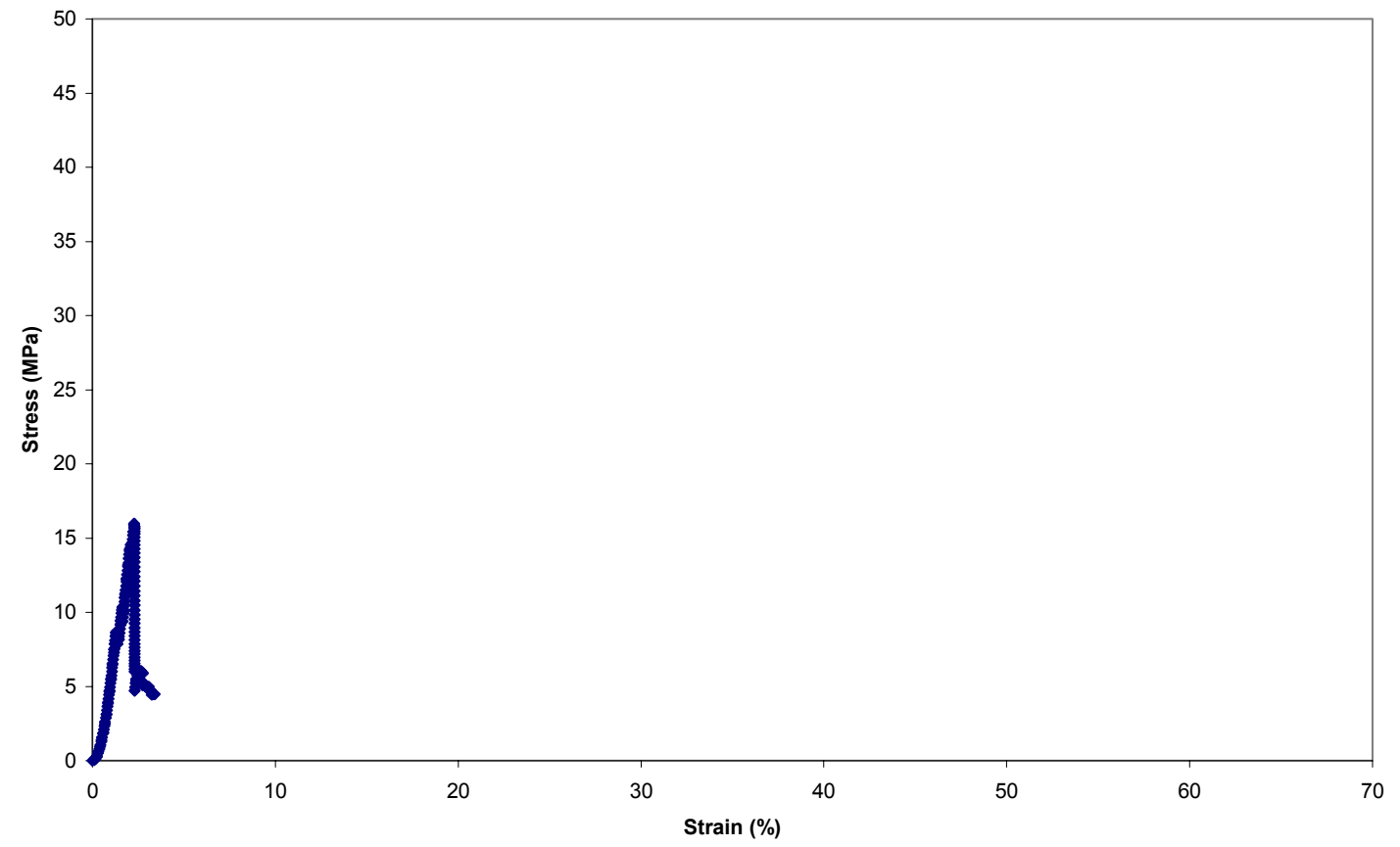

20.1.10

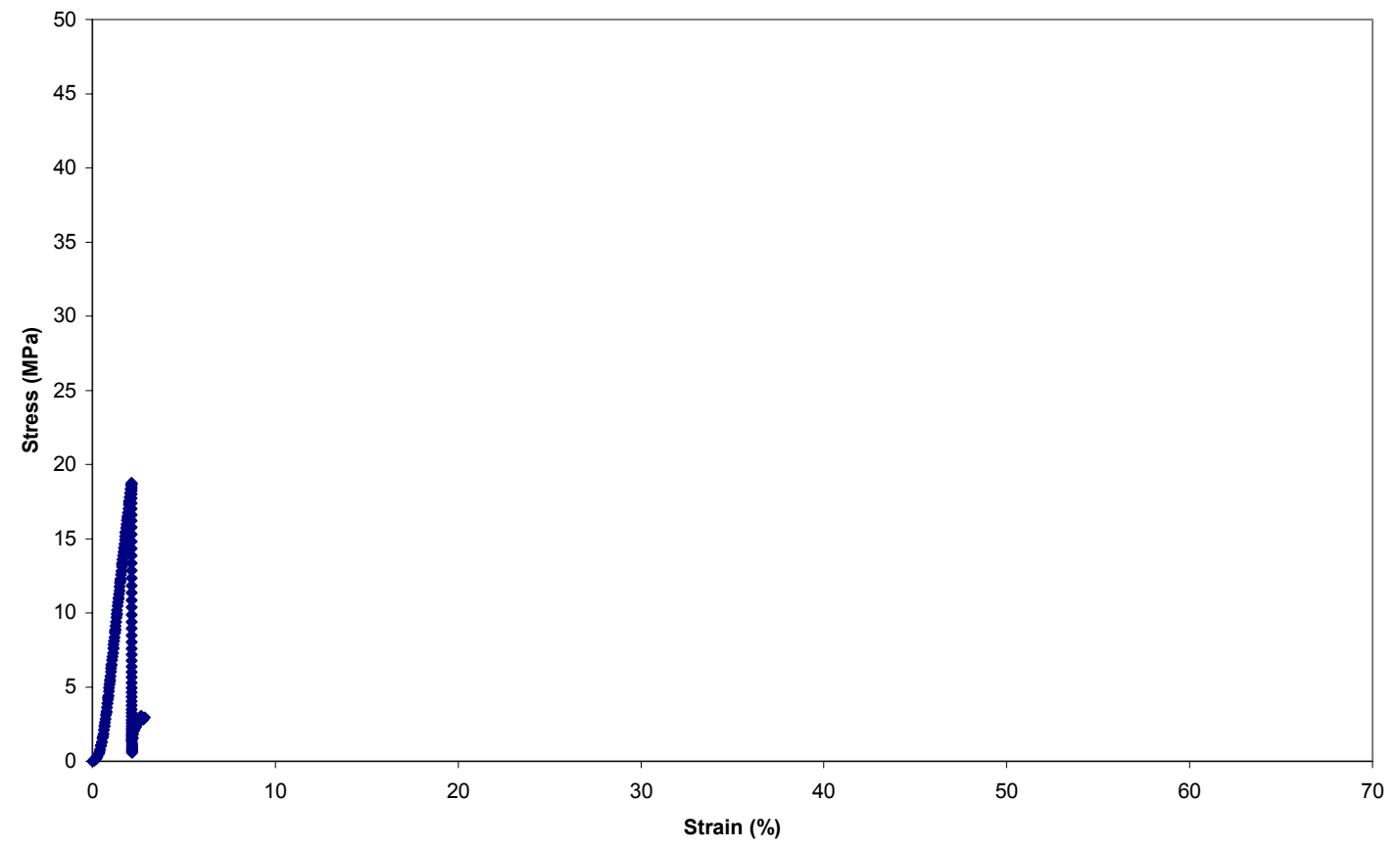

Figure A.45. Stress-Strain Plots for Samples 9 and 10 of Test 20.1. 
20.2.1

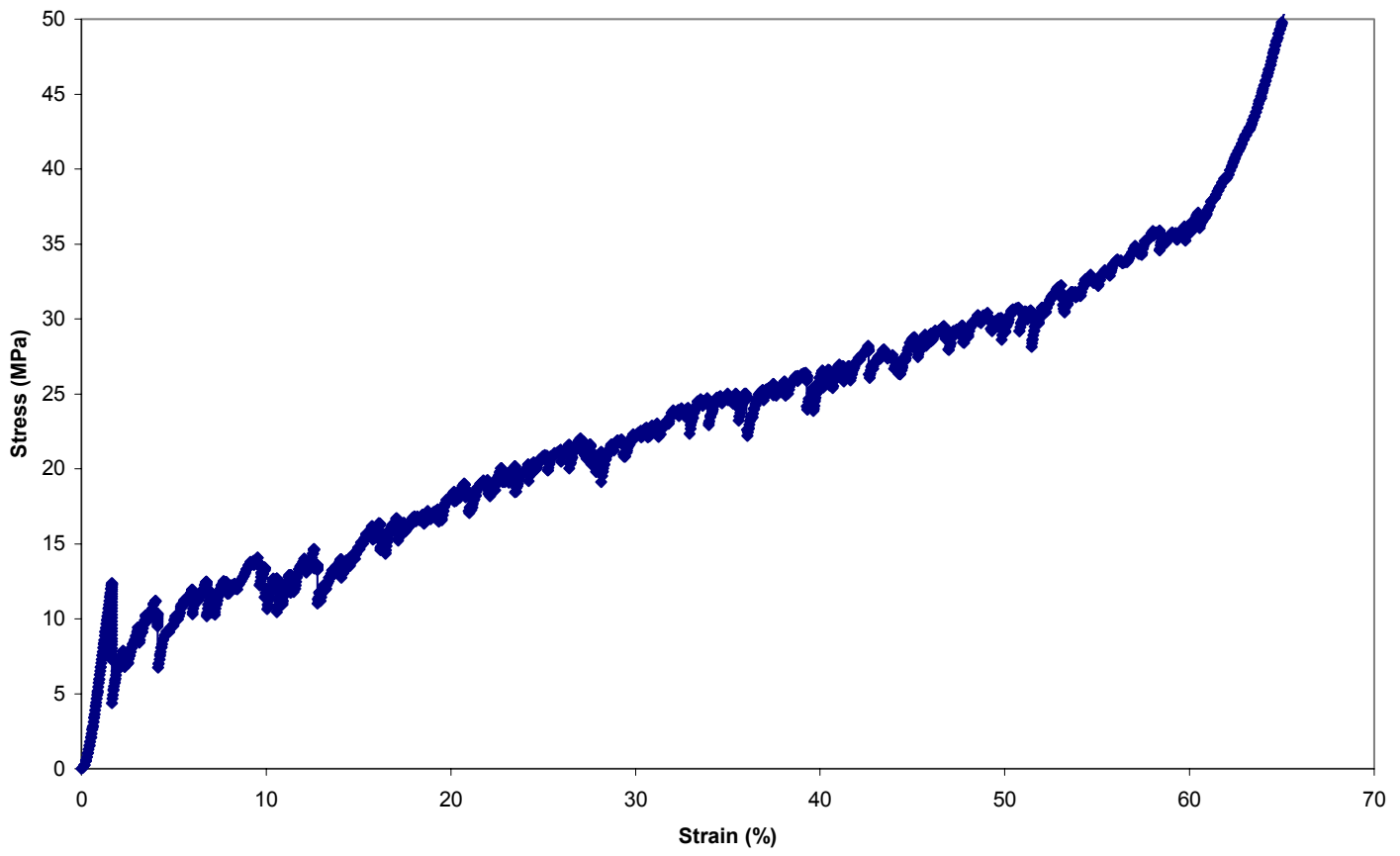

20.2.2

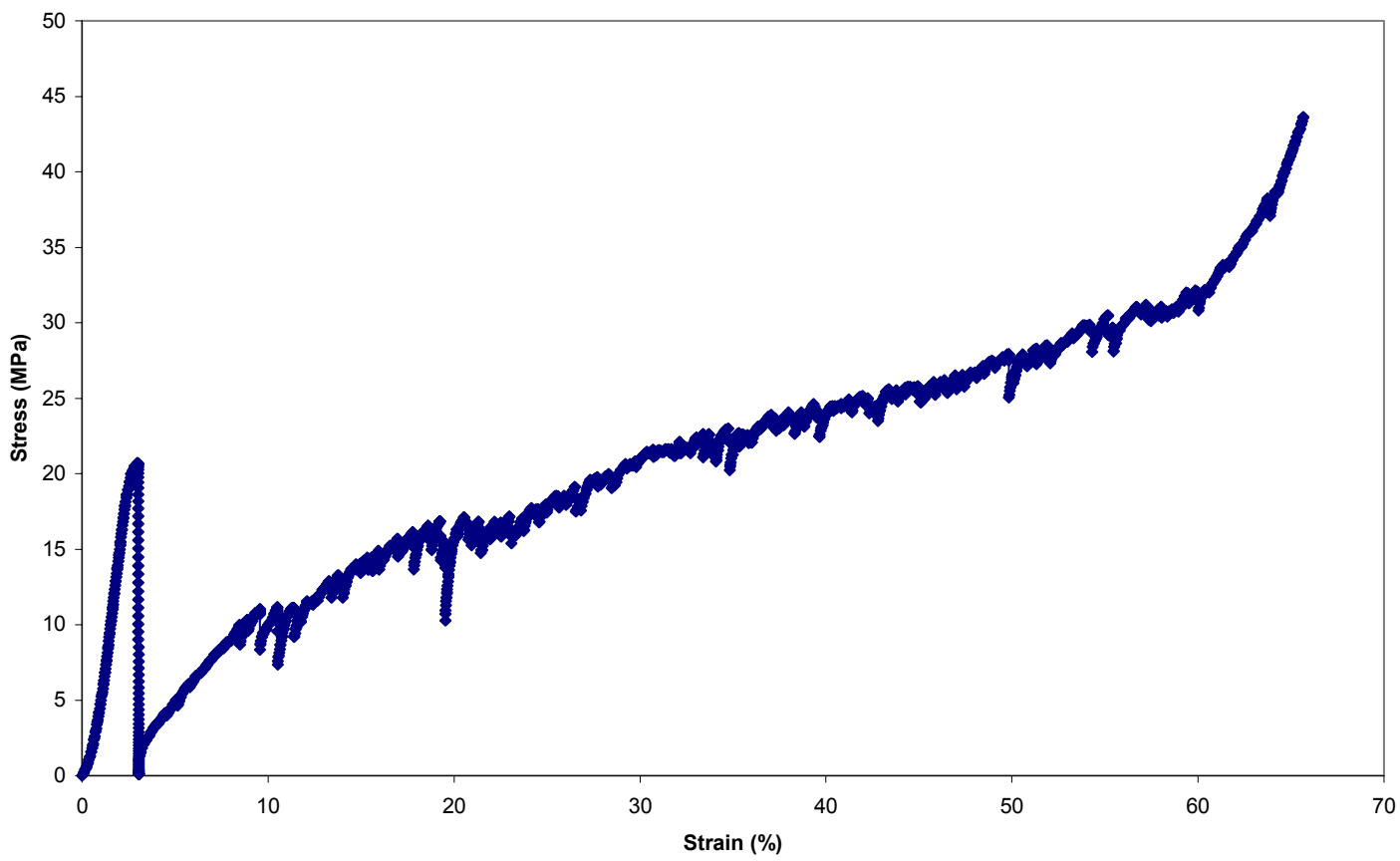

Figure A.46. Stress-Strain Plots for Samples 1 and 2 of Test 20.2. 


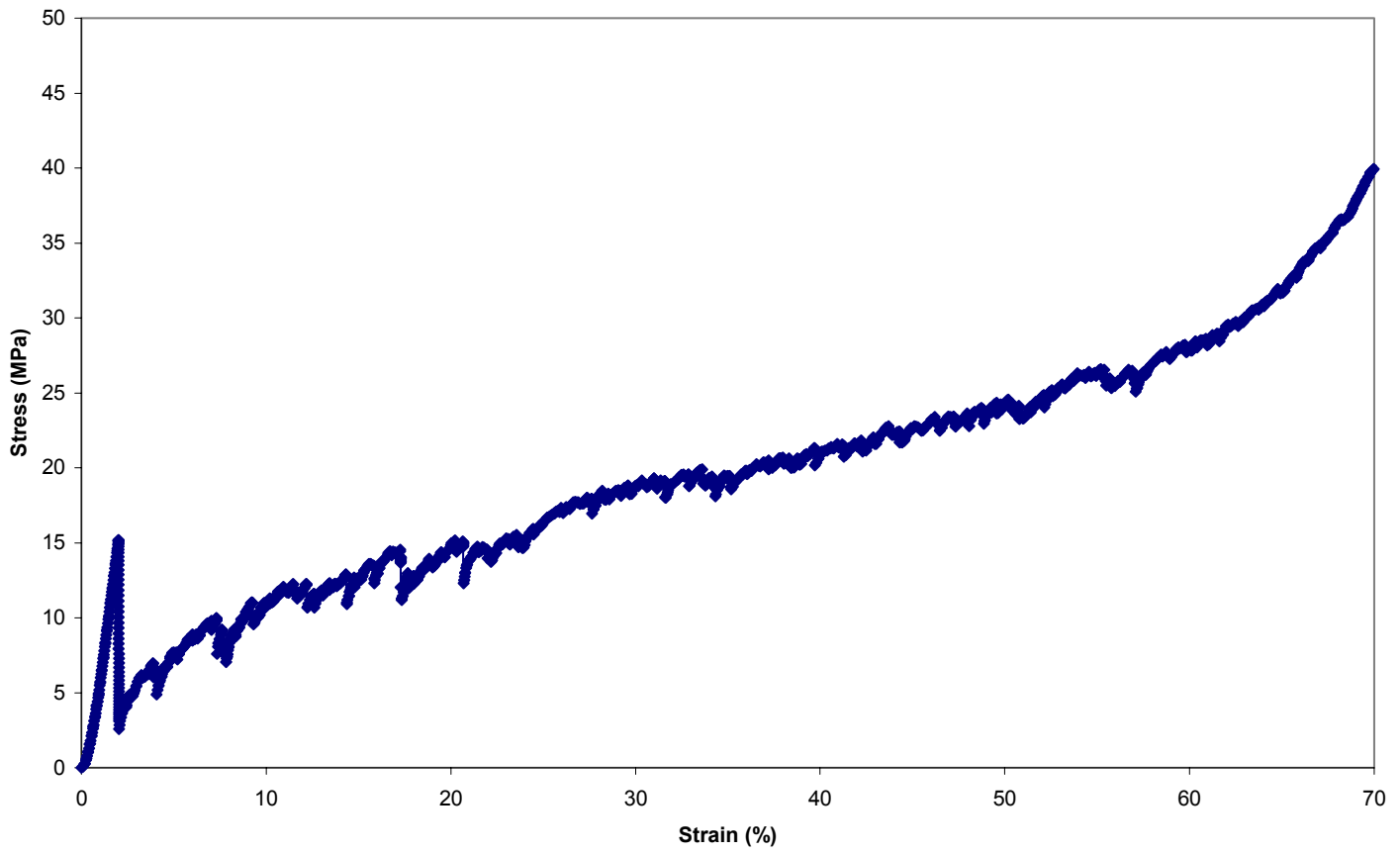

20.2.5

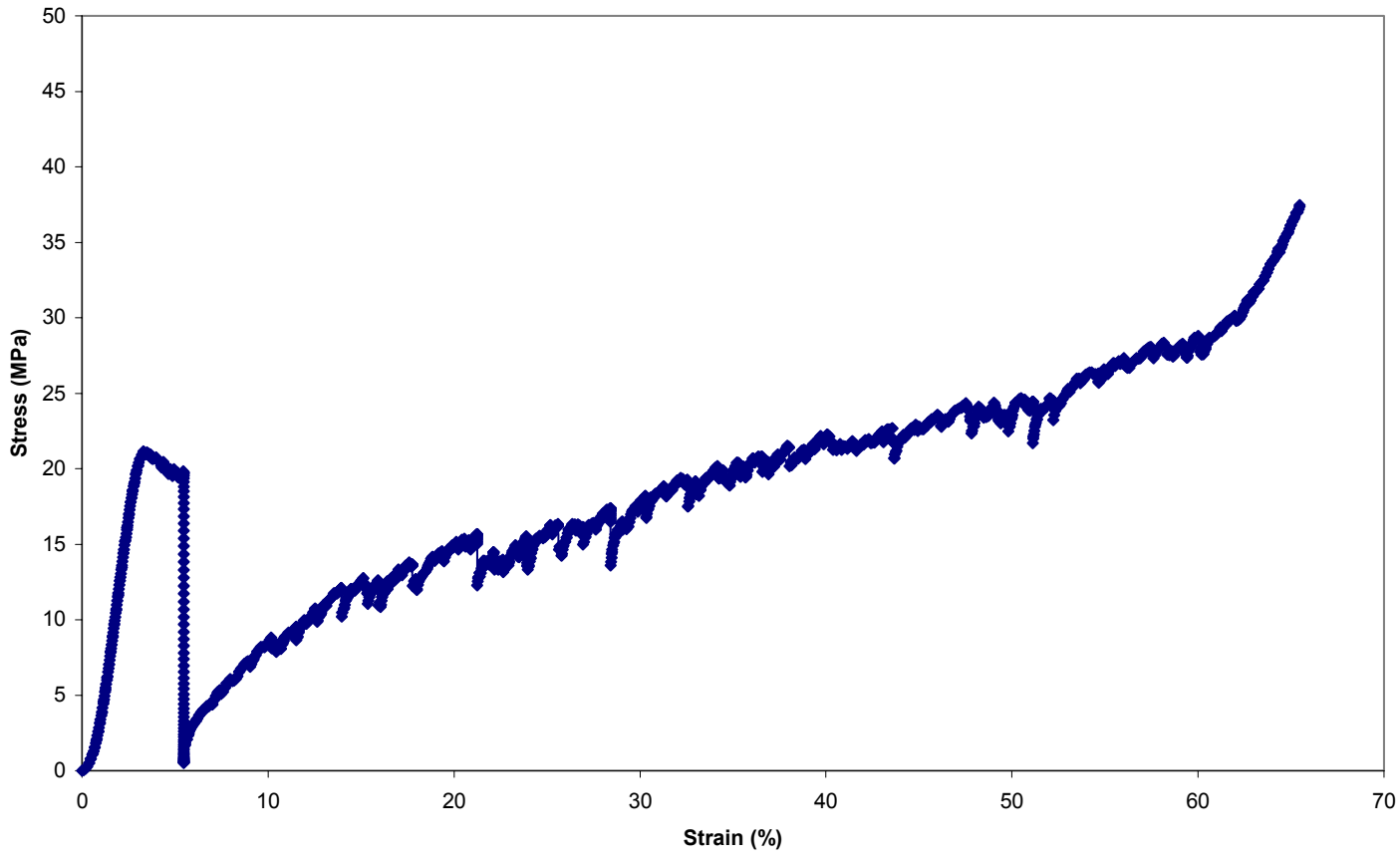

Figure A.47. Stress-Strain Plots for Samples 3 and 5 of Test 20.2. 


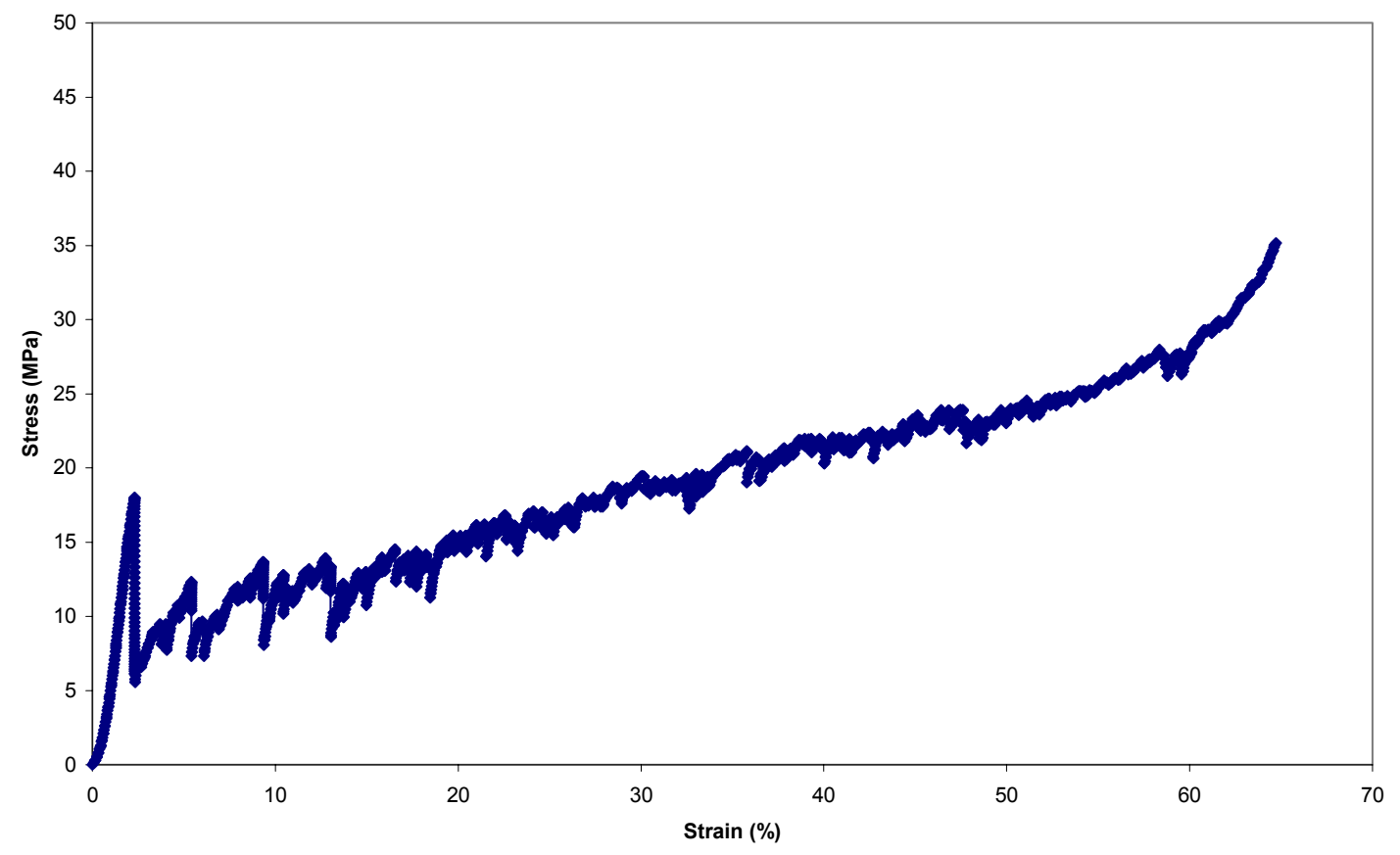

20.2.7

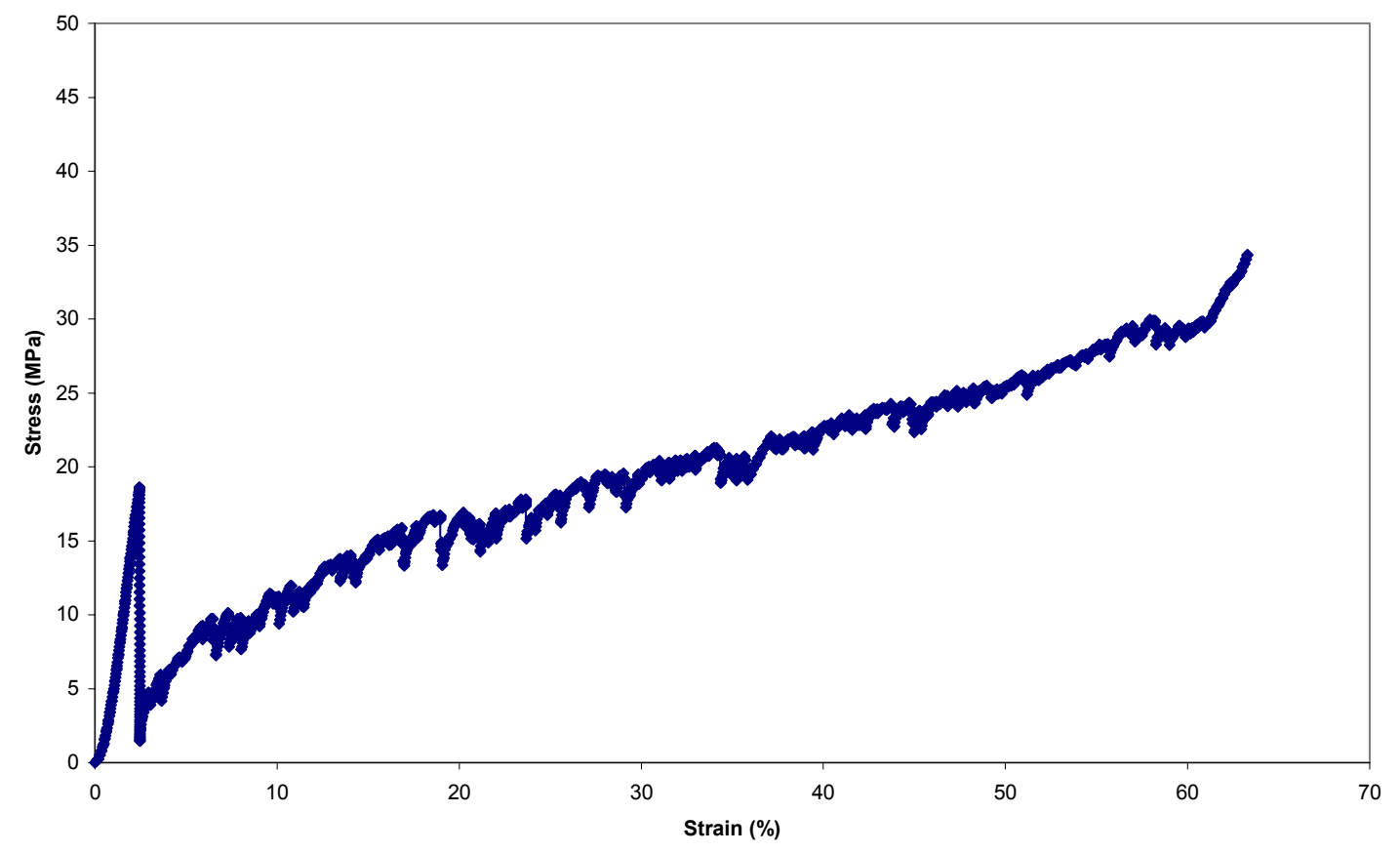

Figure A.48. Stress-Strain Plots for Samples 6 and 7 of Test 20.2. 


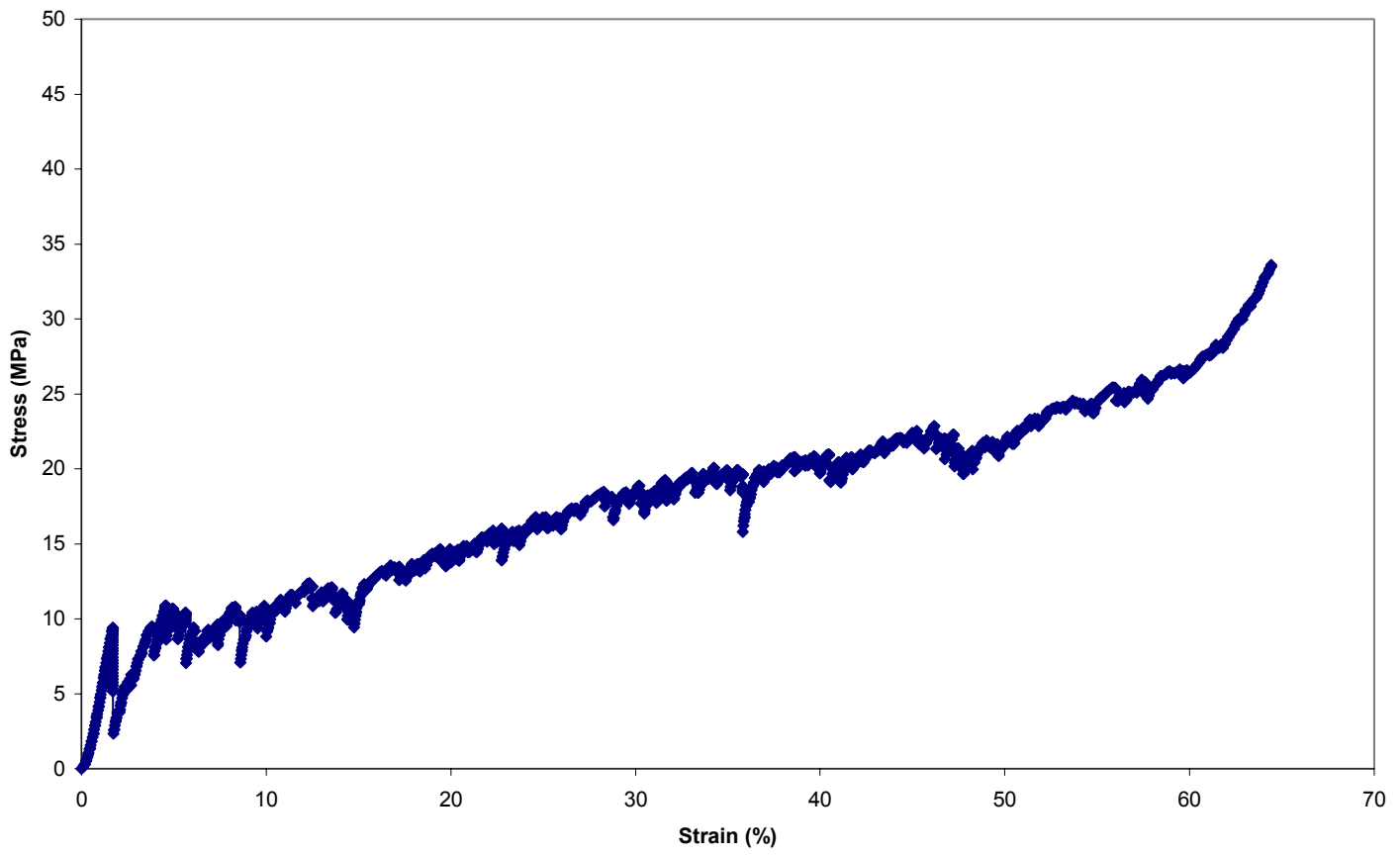

20.2.9

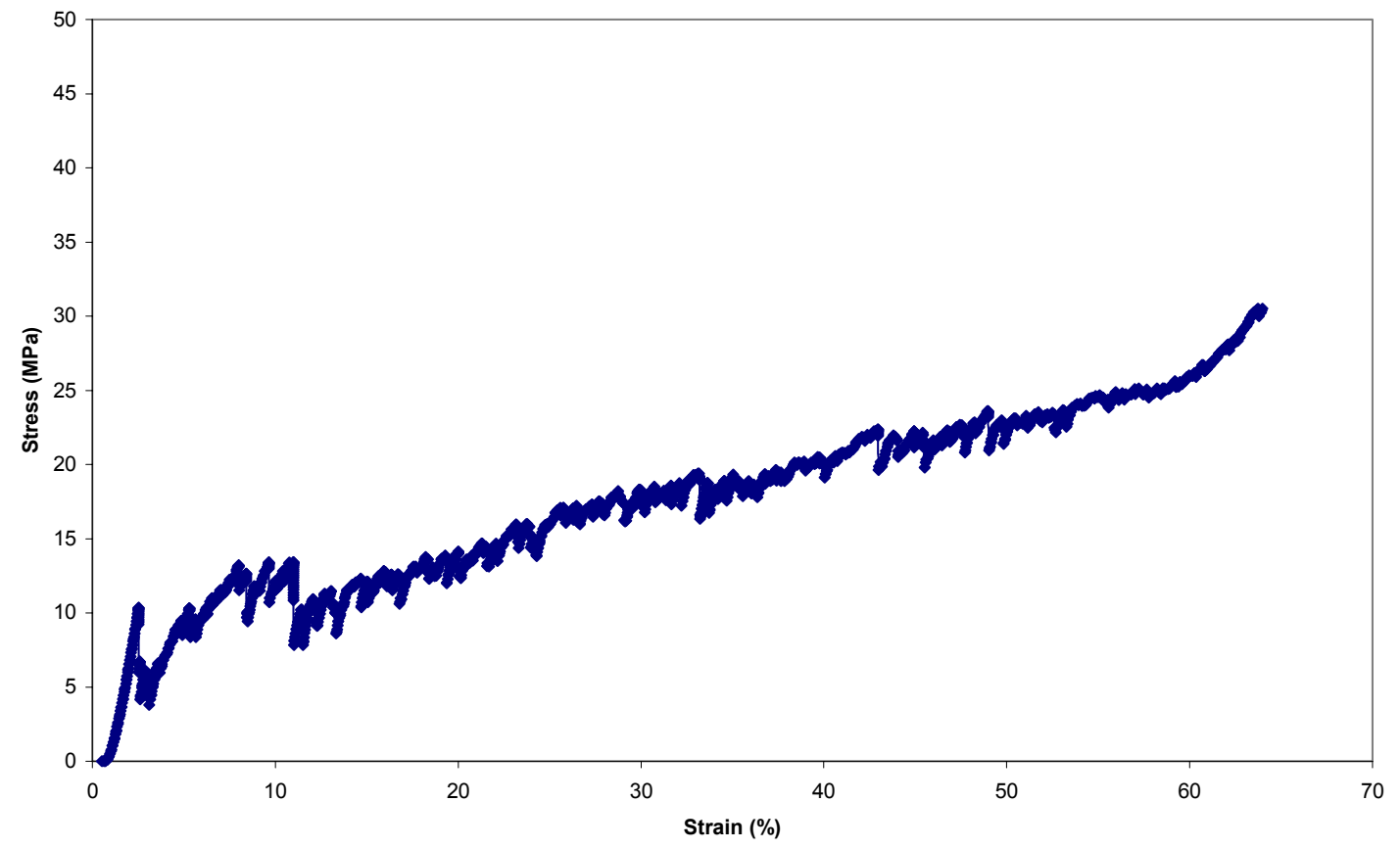

Figure A.49. Stress-Strain Plots for Samples 8 and 9 of Test 20.2. 
20.2.10

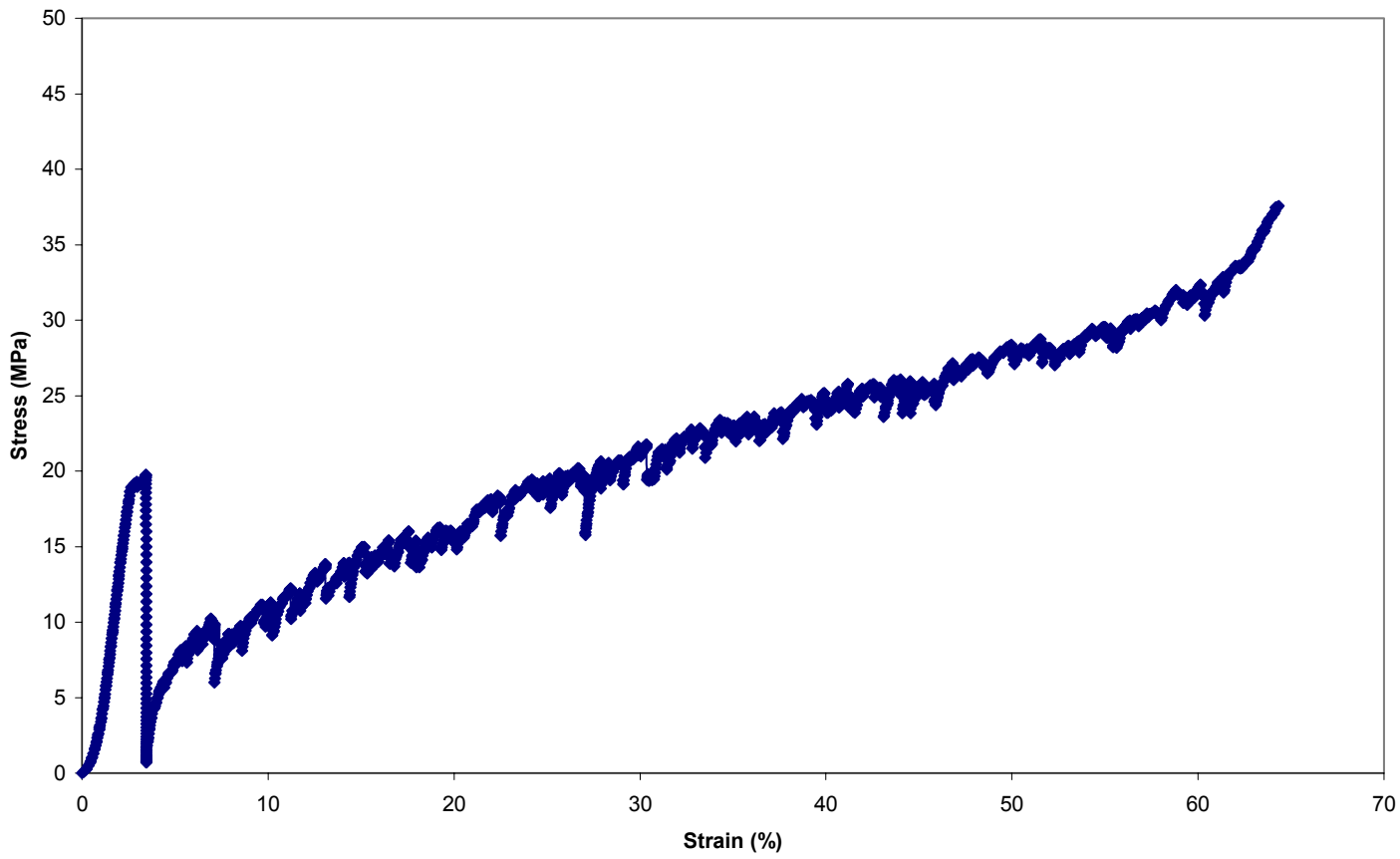

Figure A.50. Stress-Strain Plot for Sample 10 of Test 20.2. 


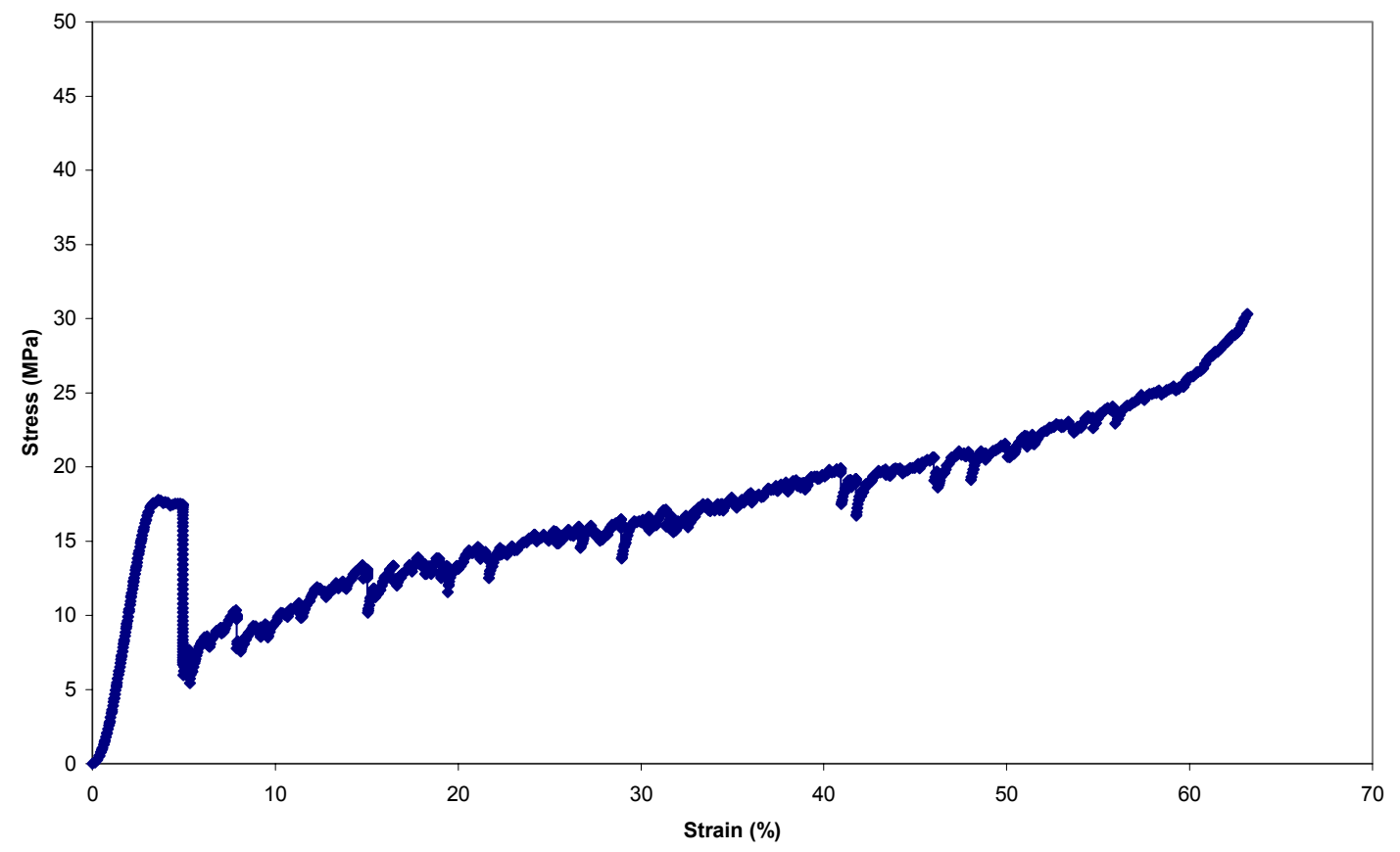

20.3.2

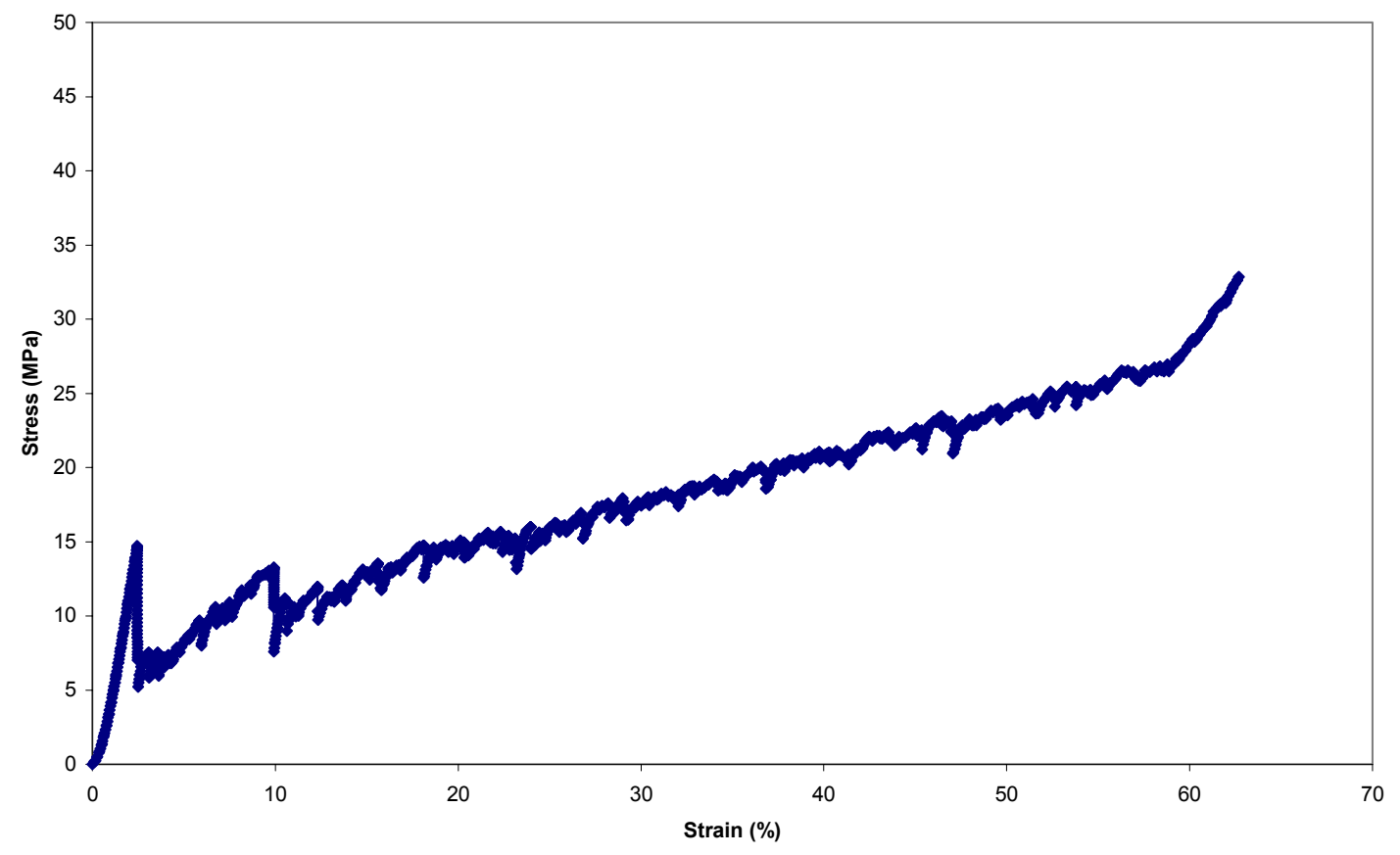

Figure A.51. Stress-Strain Plots for Samples 1 and 2 of Test 20.3. 


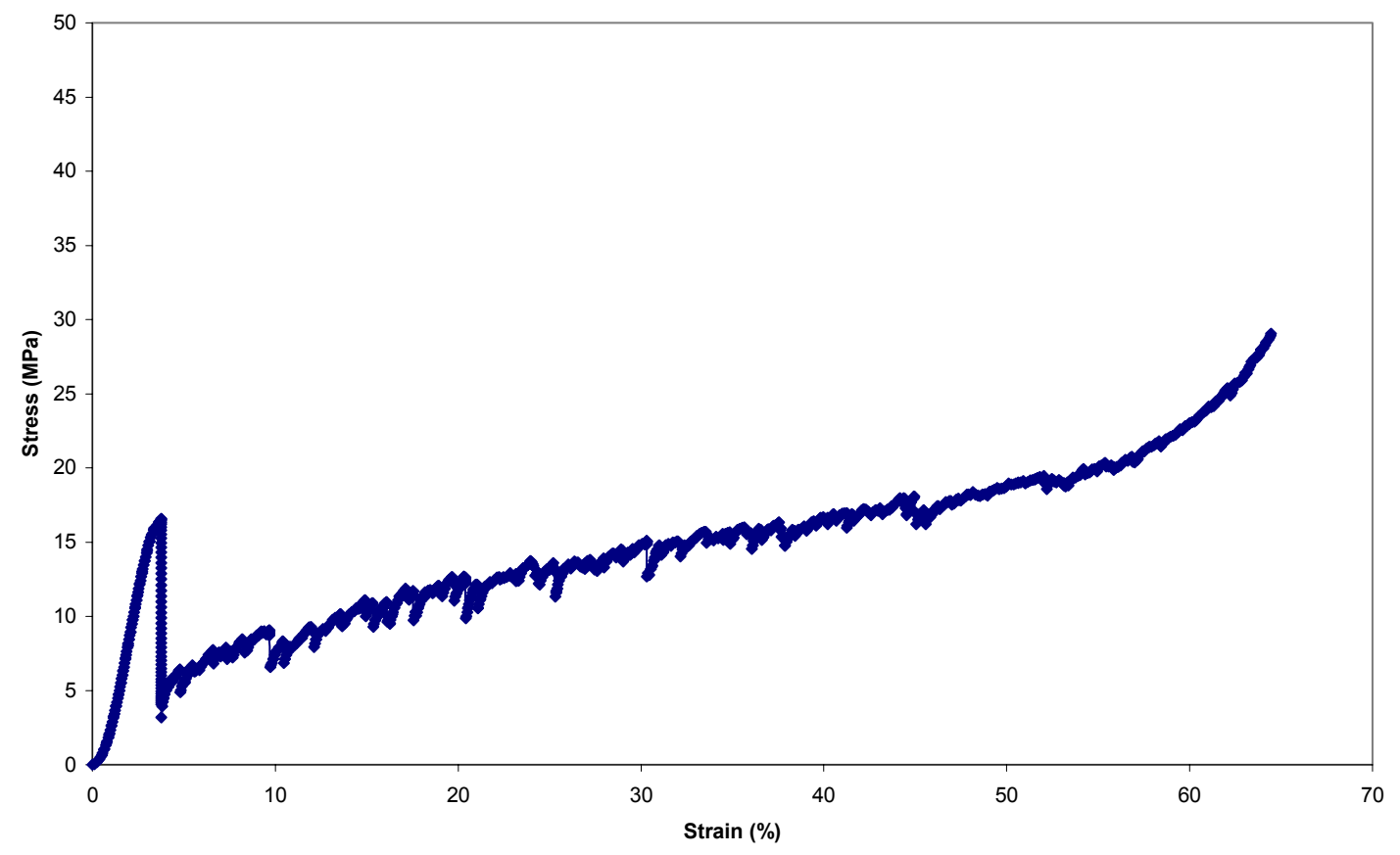

20.3.4

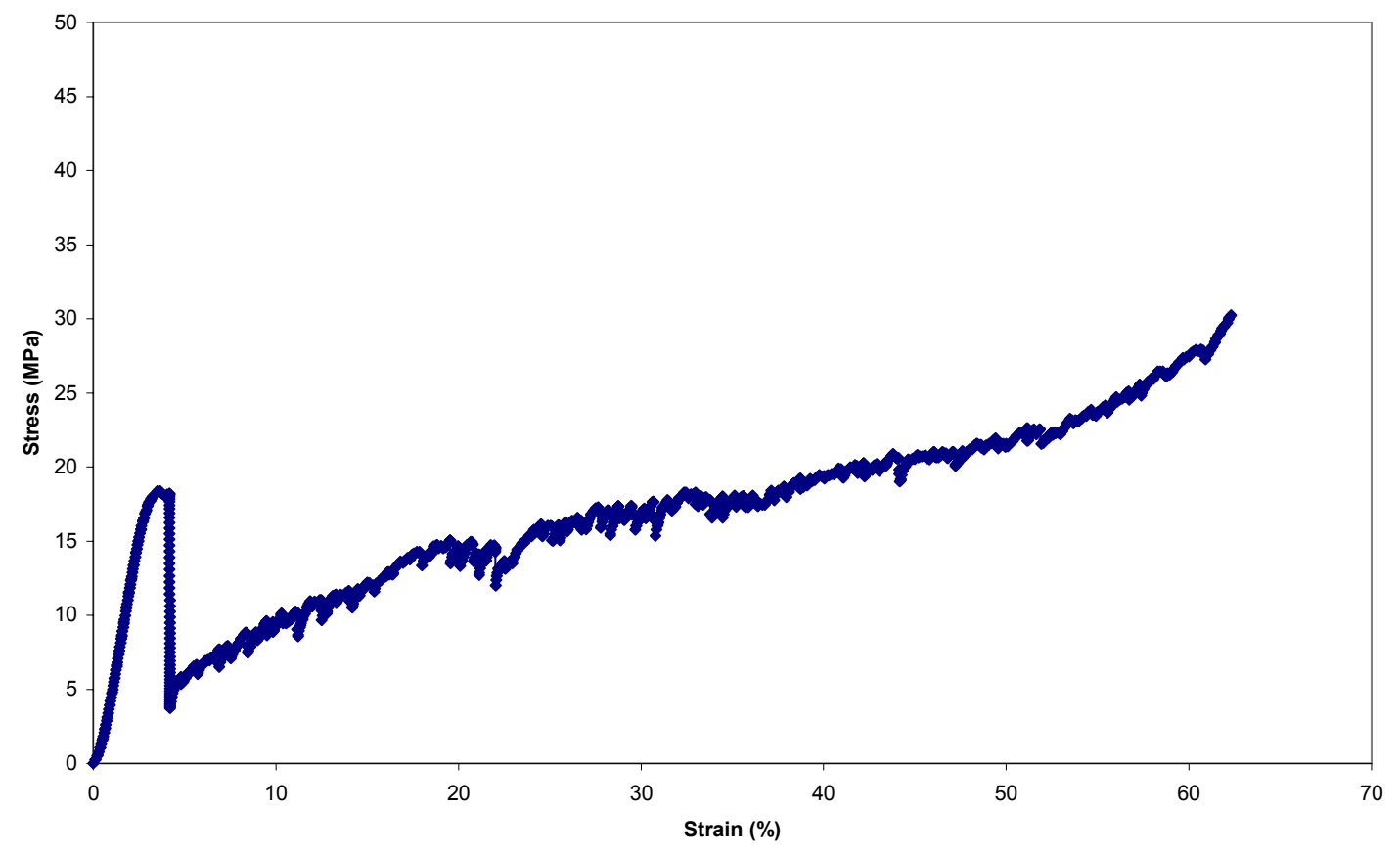

Figure A.52. Stress-Strain Plots for Samples 3 and 4 of Test 20.3. 


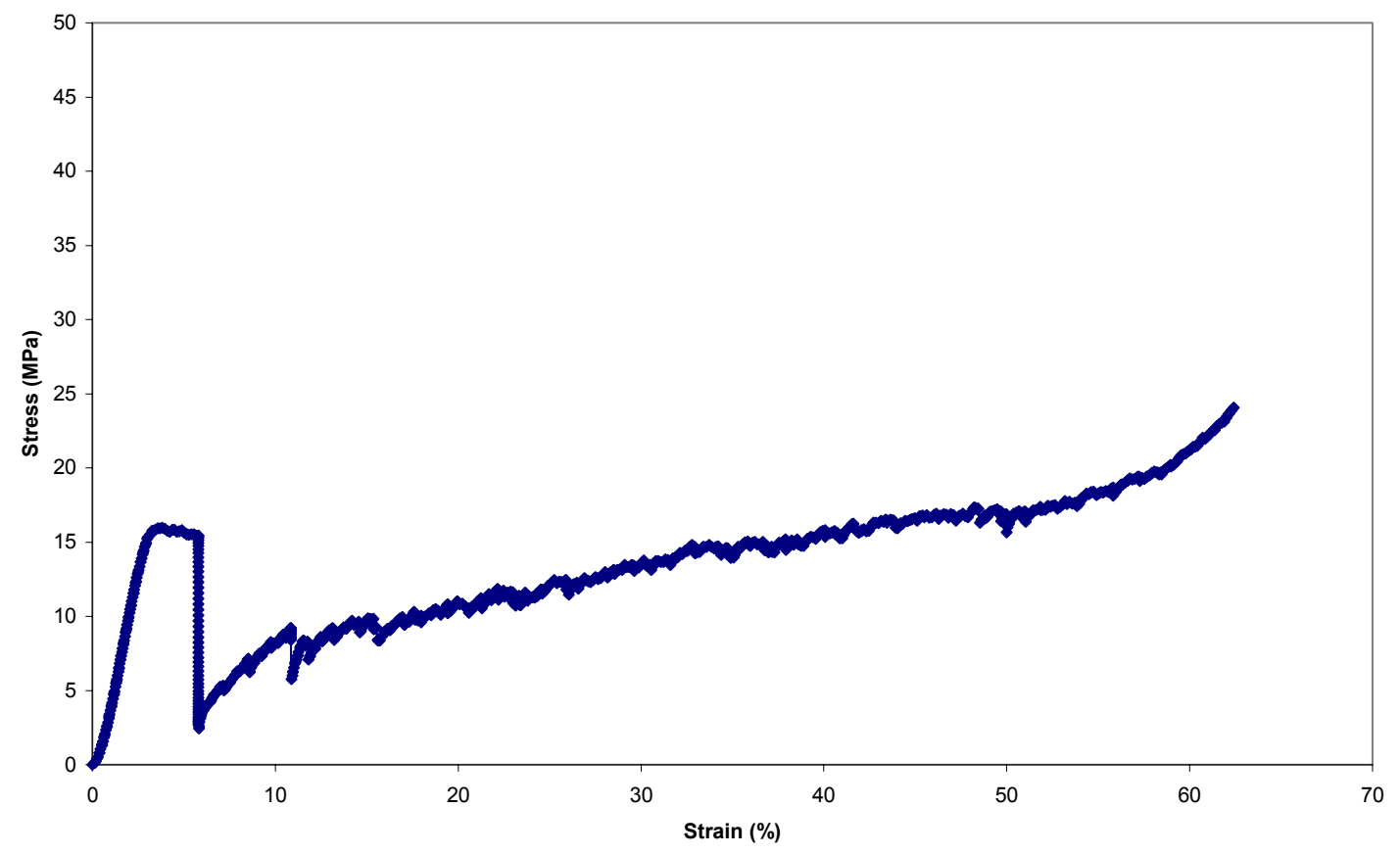

20.3.6

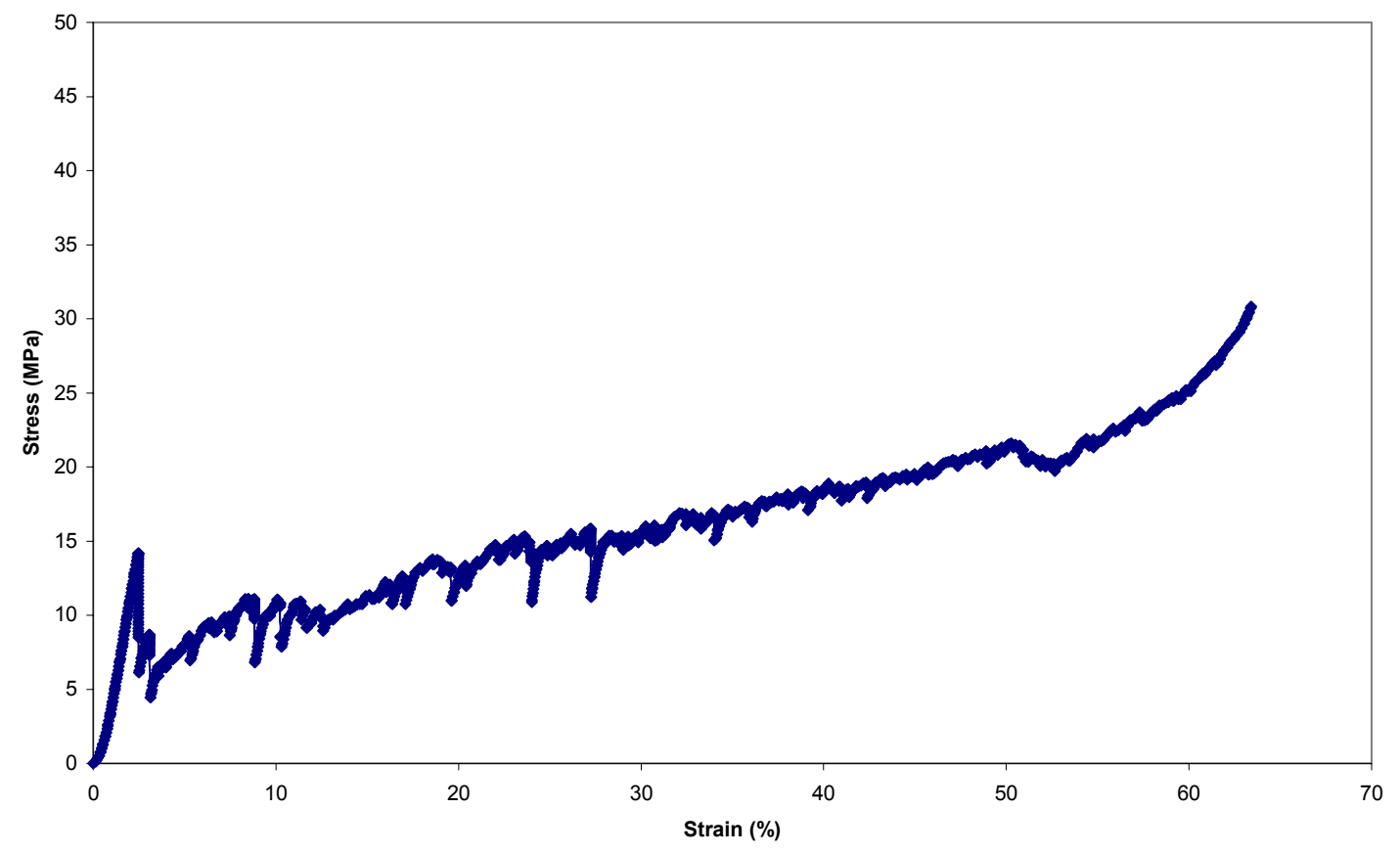

Figure A.53. Stress-Strain Plots for Samples 5 and 6 of Test 20.3. 


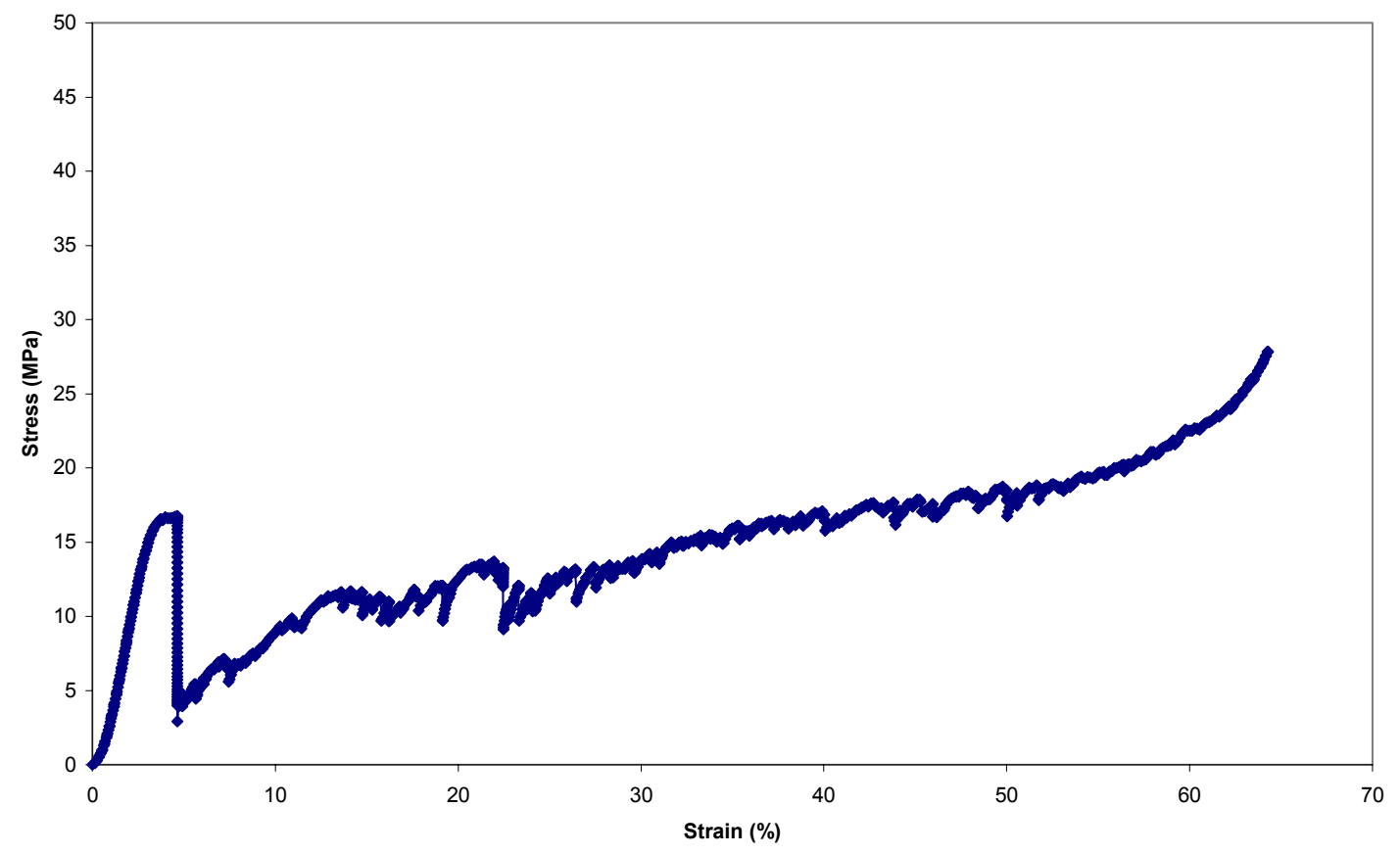

20.3.8

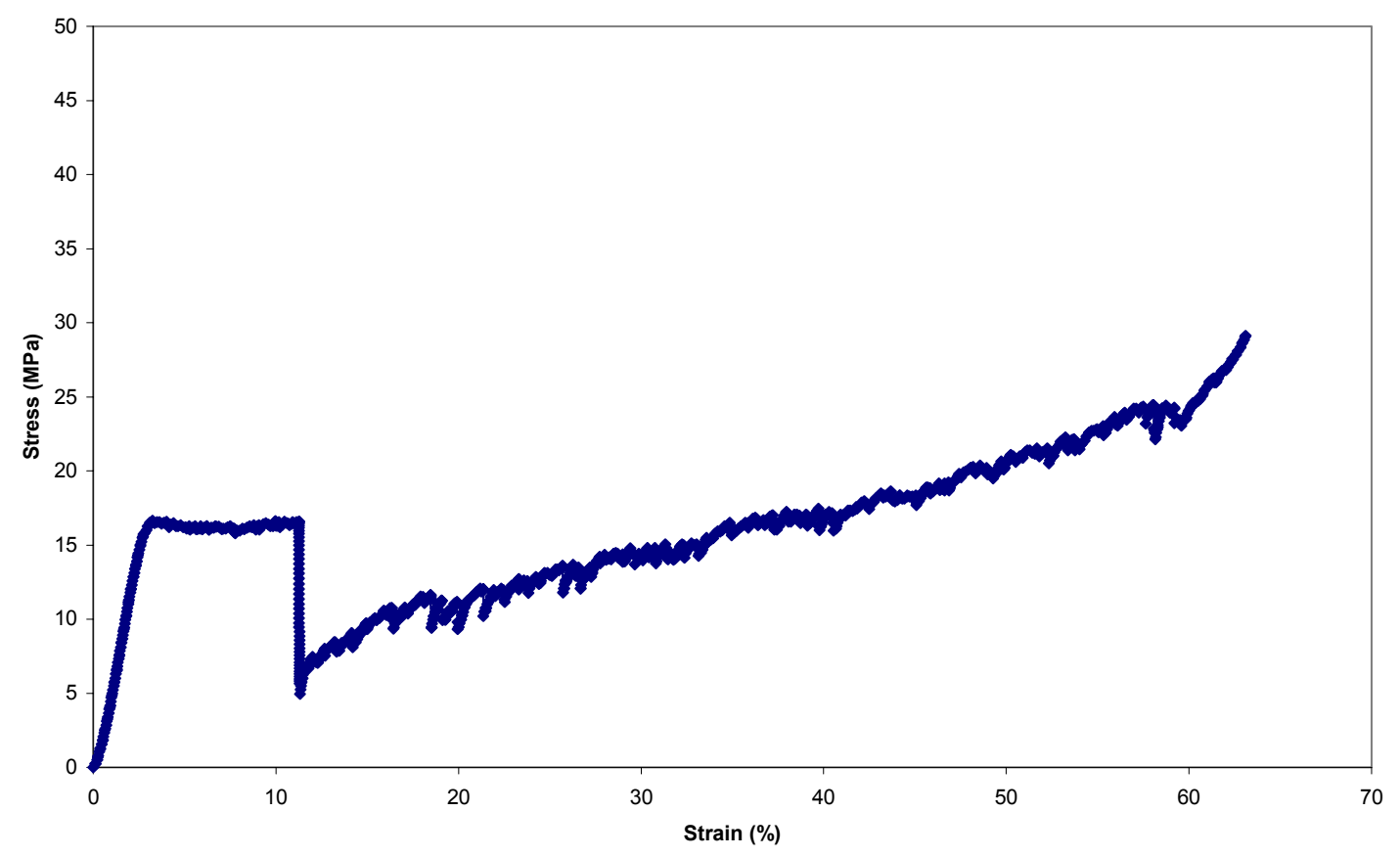

Figure A.54. Stress-Strain Plots for Samples 7 and 8 of Test 20.3. 


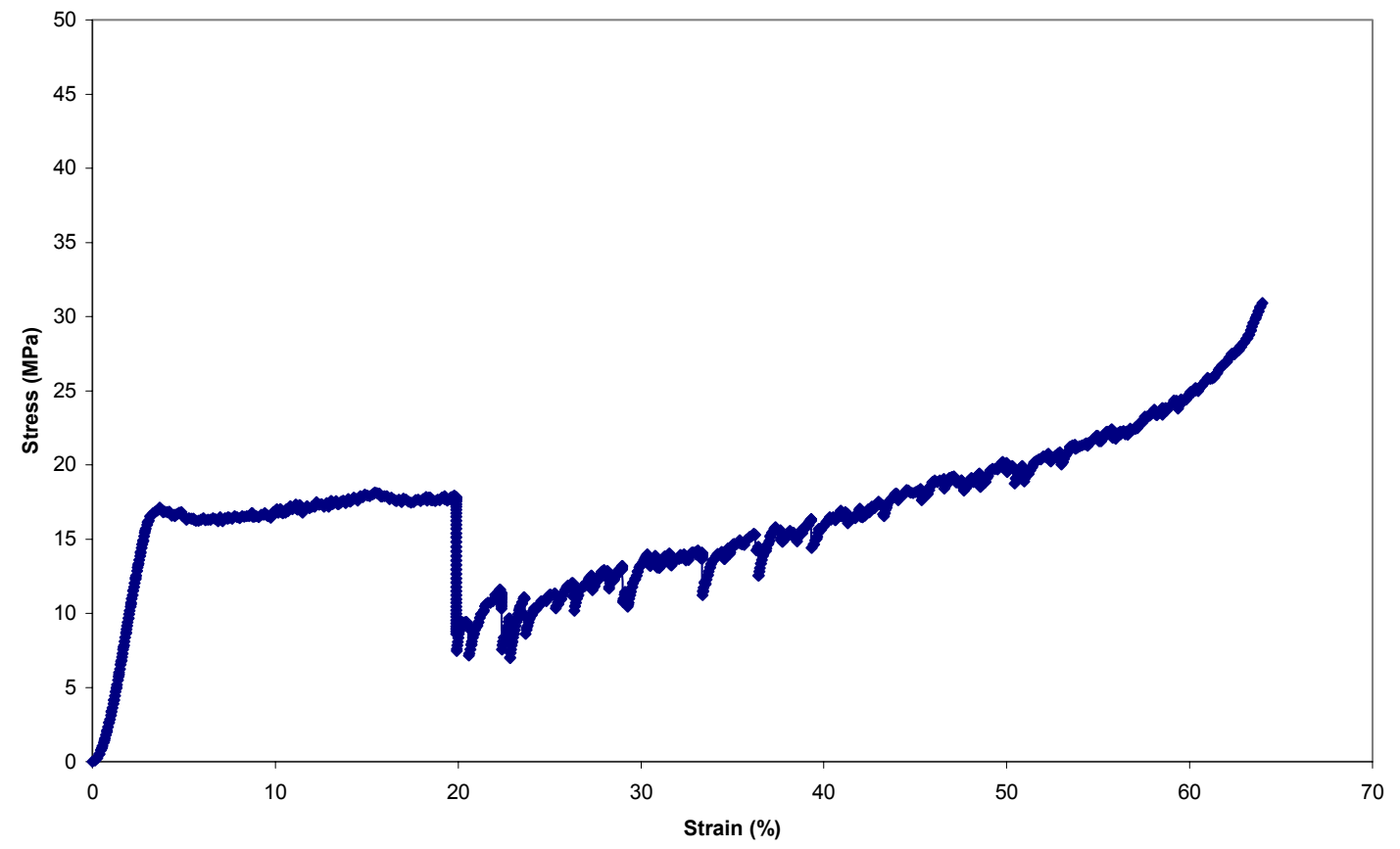

20.3.10

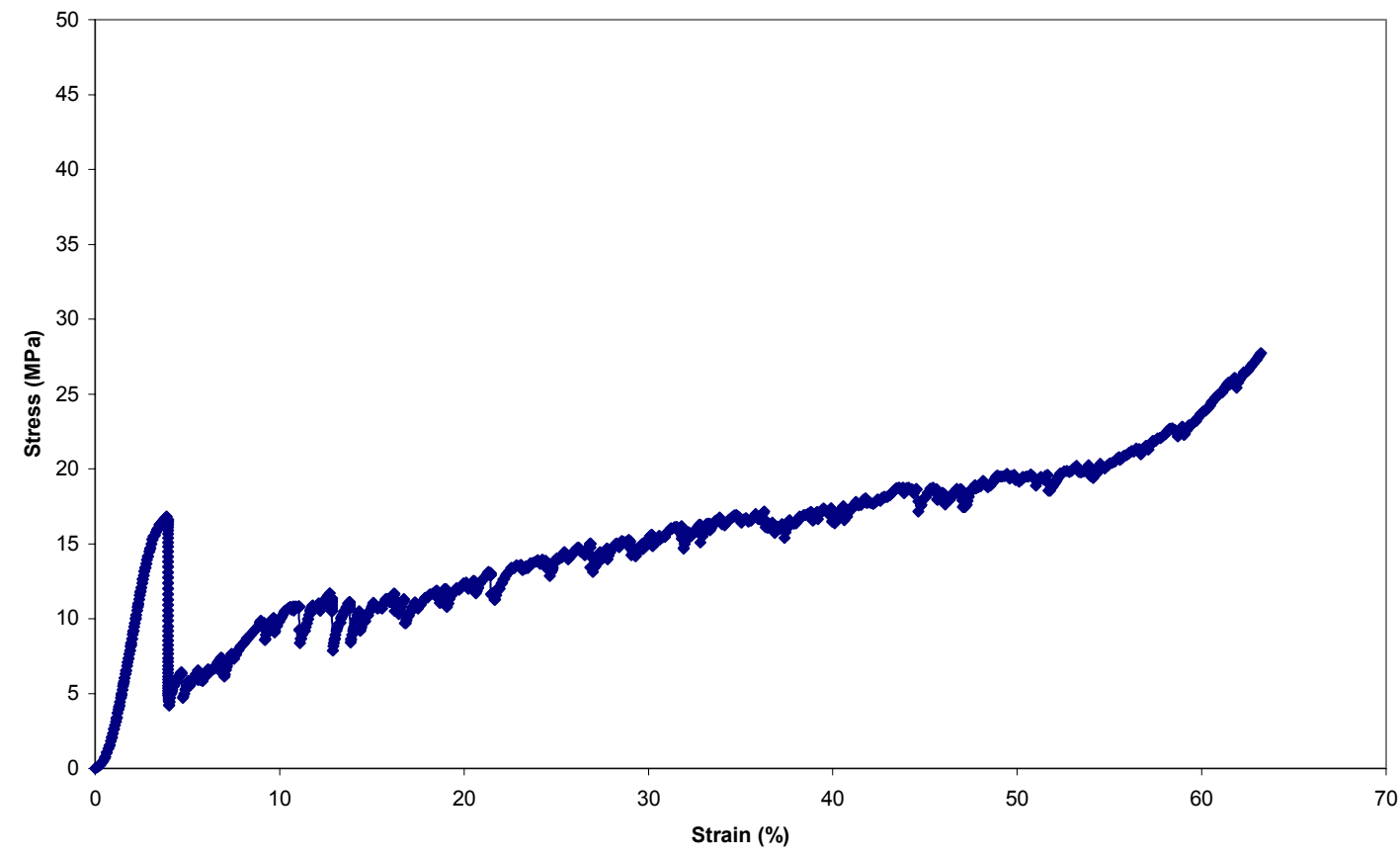

Figure A.55. Stress-Strain Plots for Samples 9 and 10 of Test 20.3 . 
MER.1.1

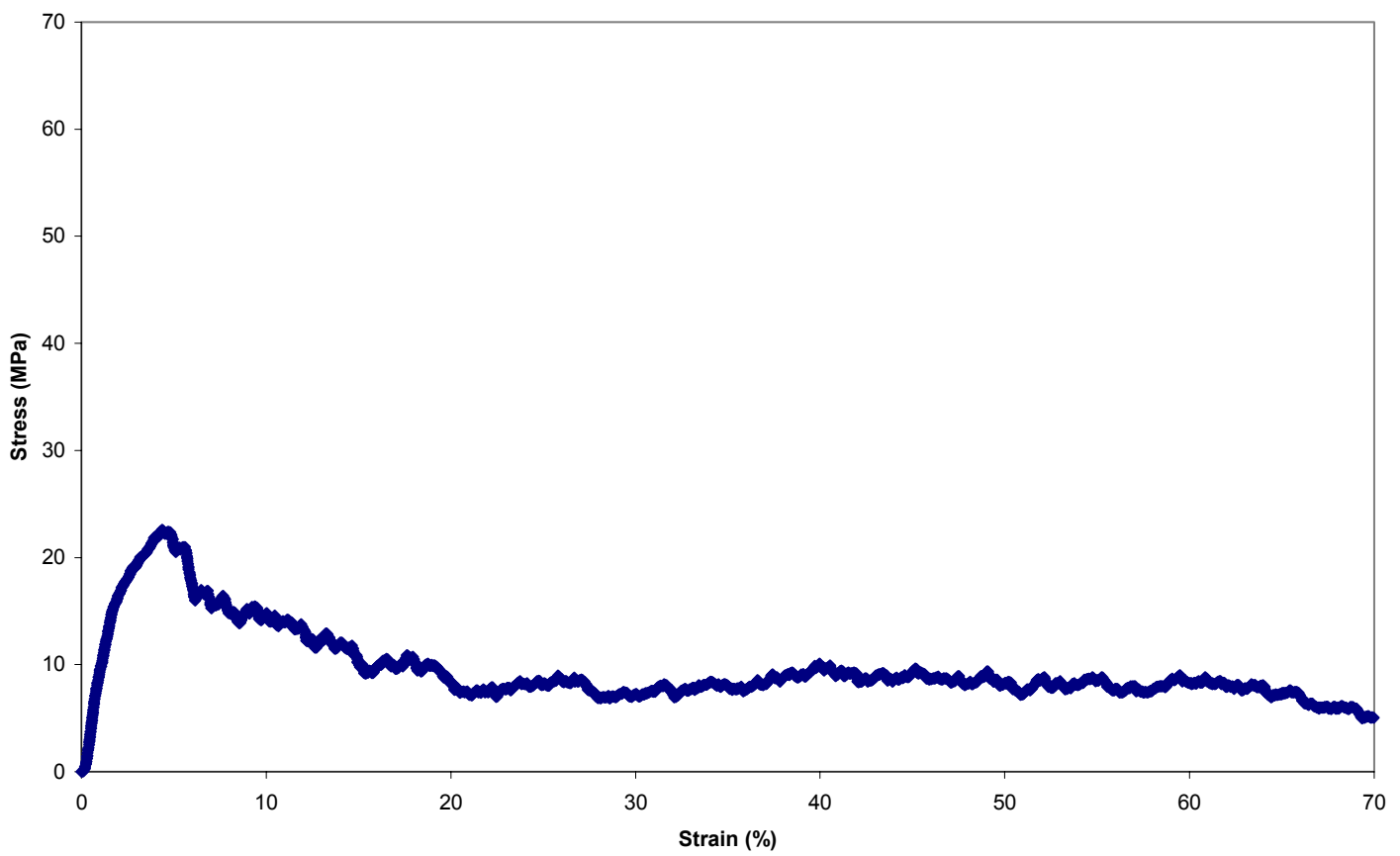

MER.1.2

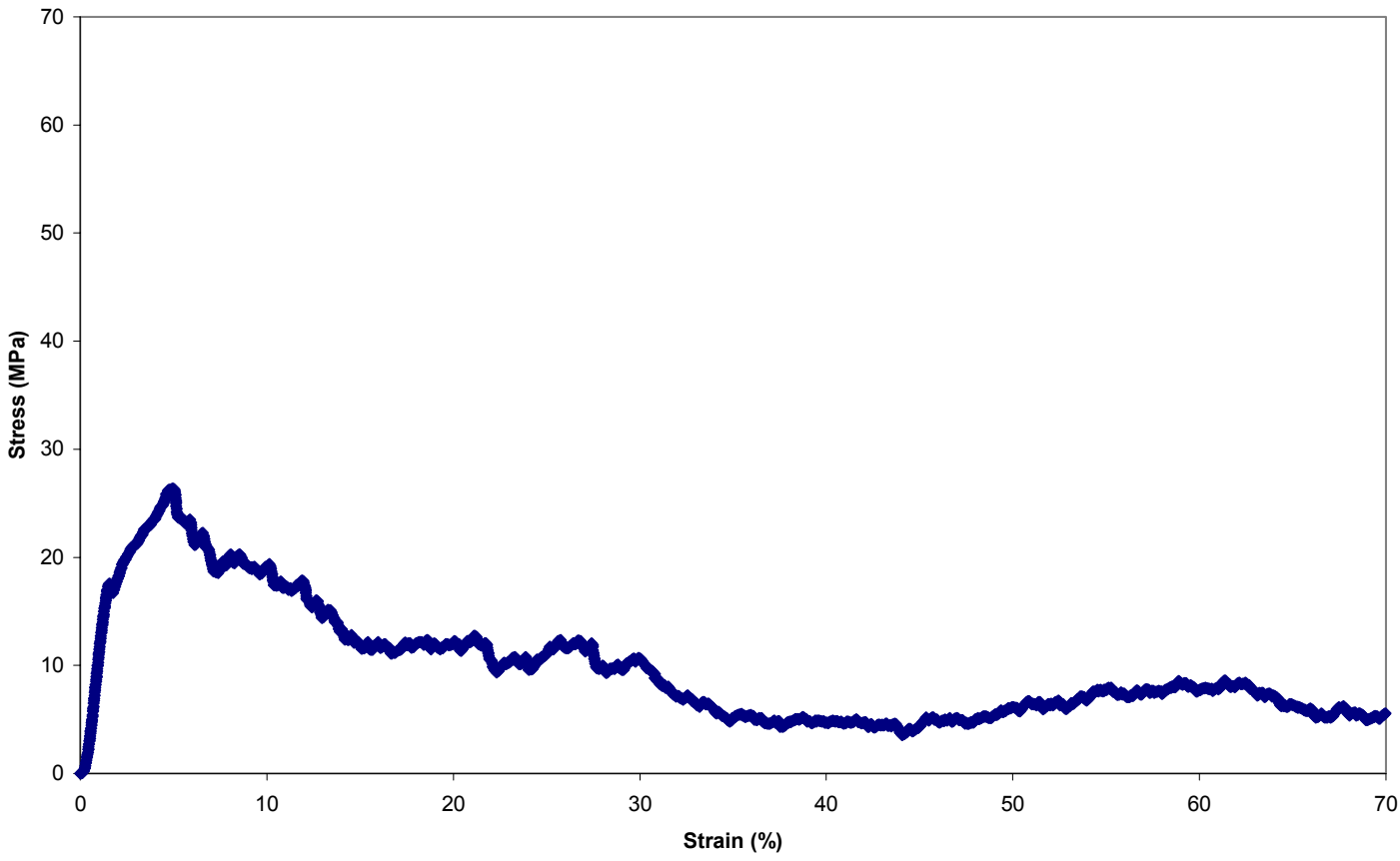

Figure A.56. Stress-Strain Plots for Samples 1 and 2 of Test MER.1. 
MER.1.3

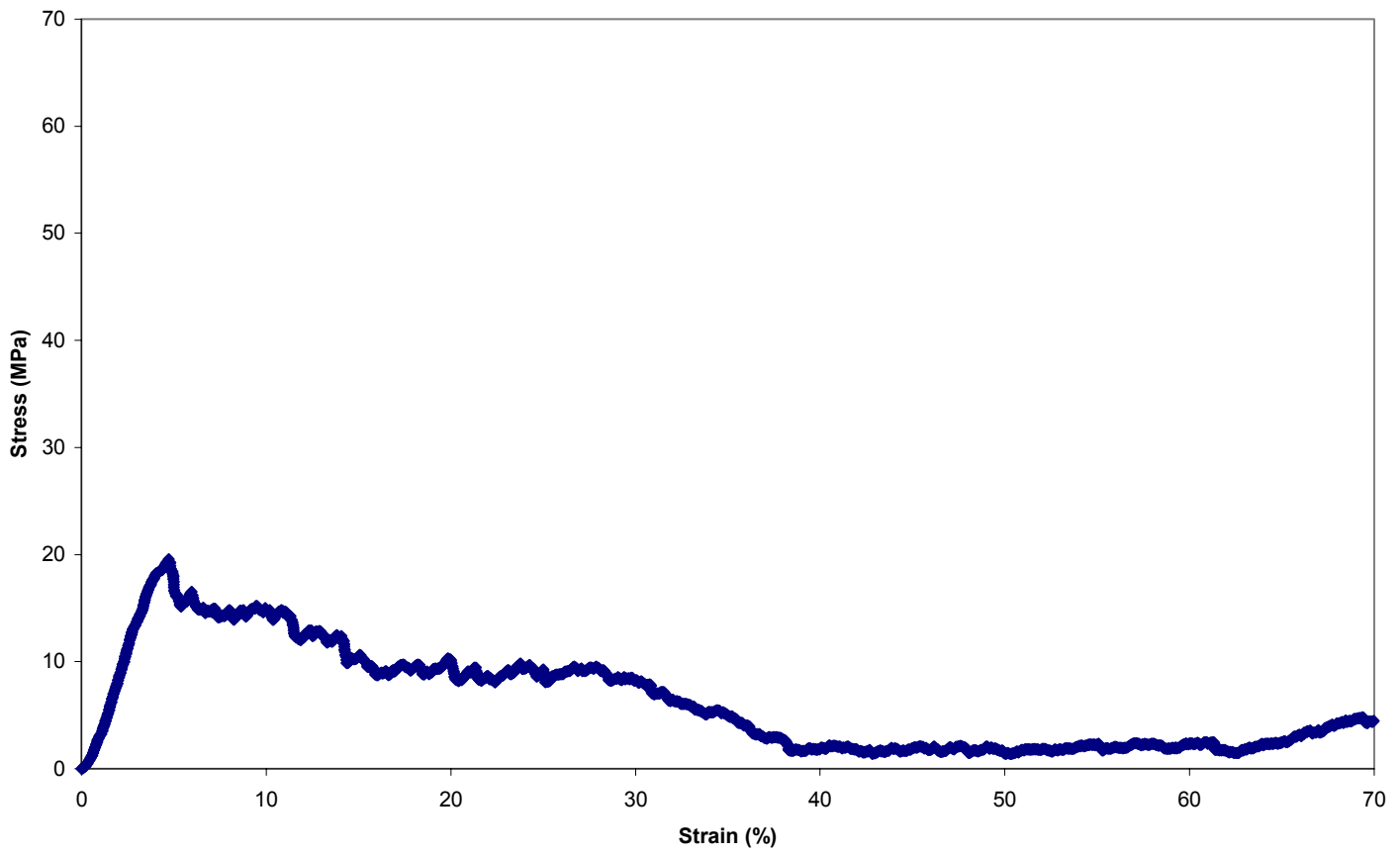

MER.2.1

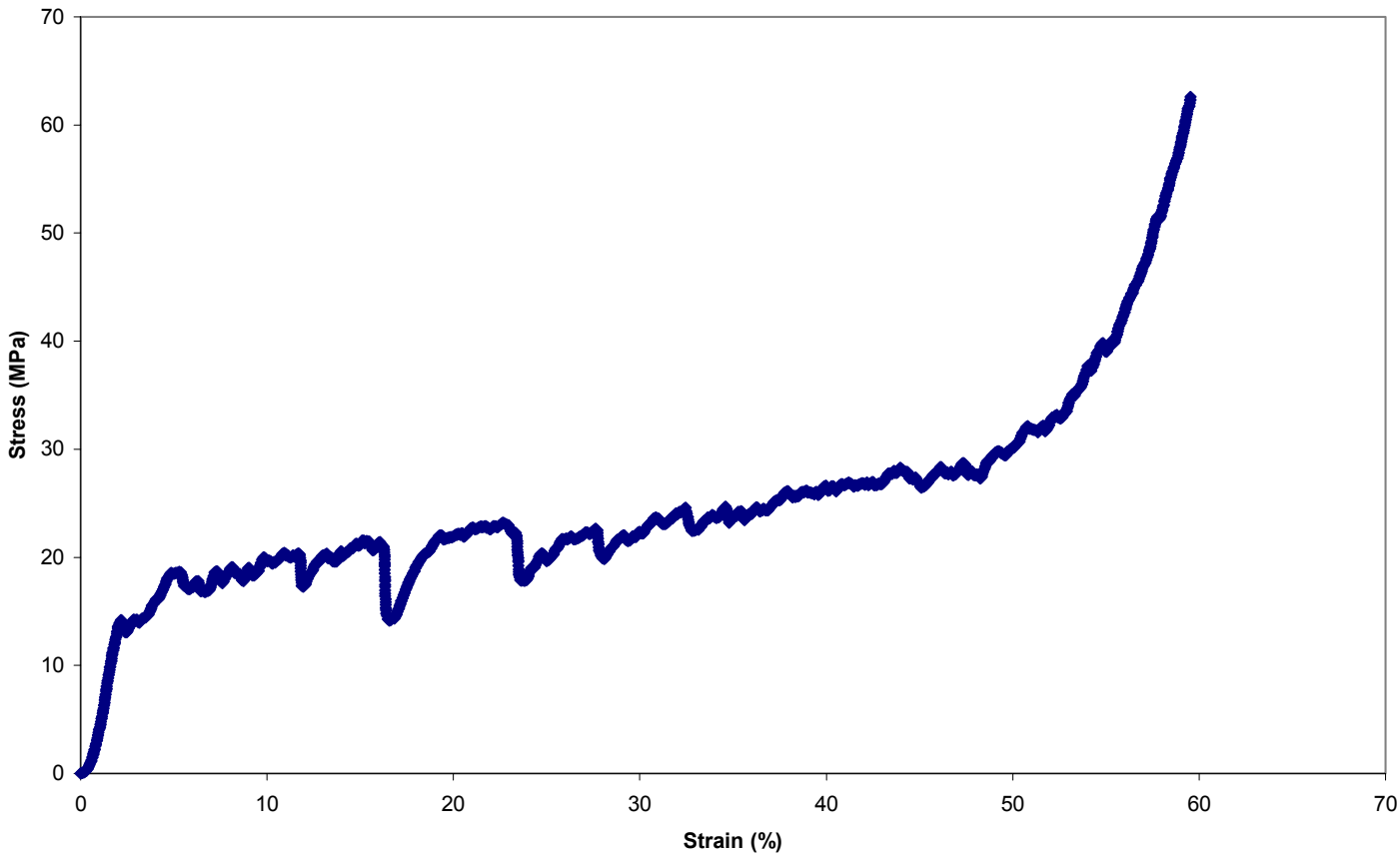

Figure A.57. Stress-Strain Plots for Sample 3 of Test MER.1 and Sample 1 of Test MER.2. 
MER.2.2

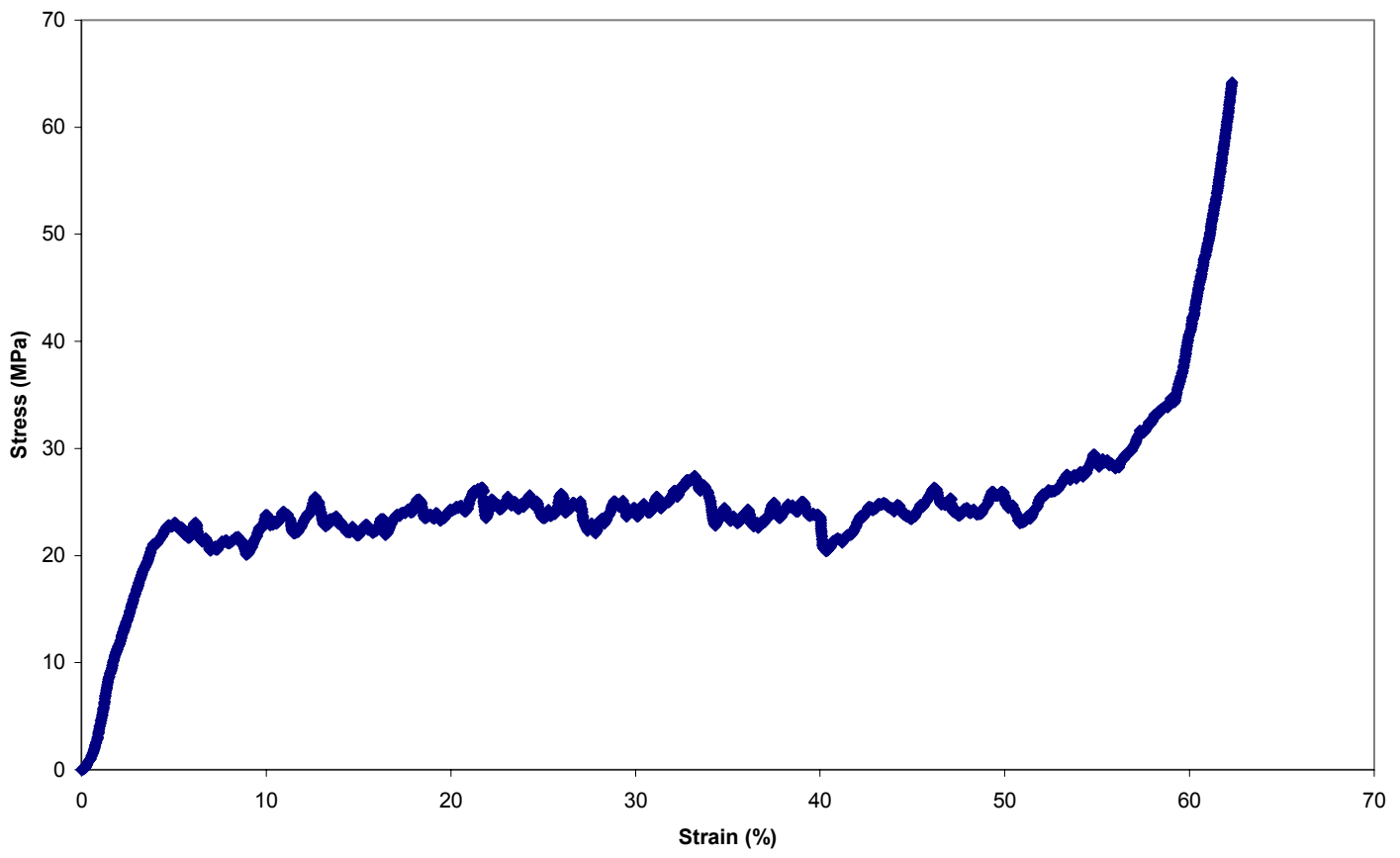

MER.2.3

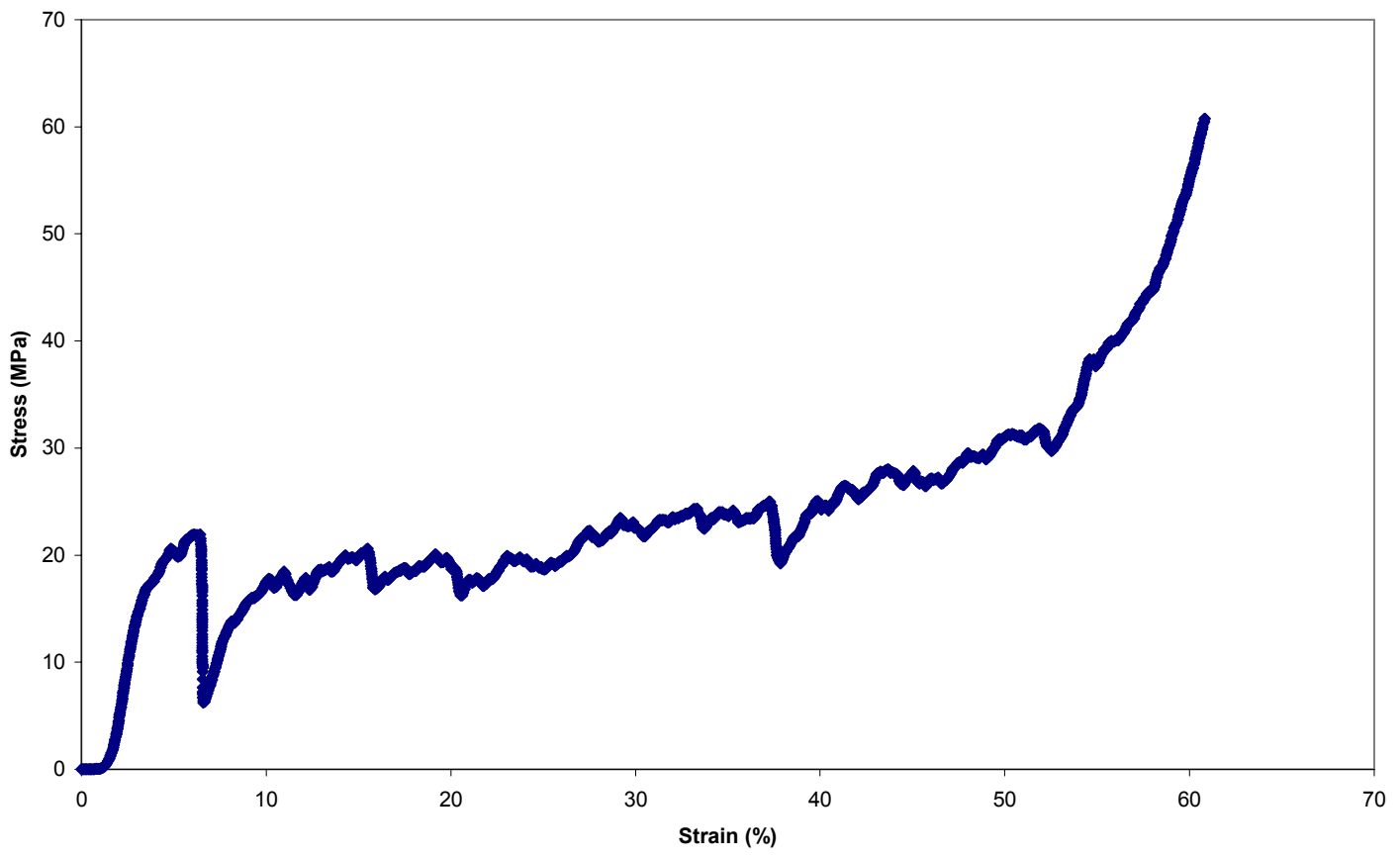

Figure A.58. Stress-Strain Plots for Samples 2 and 3 of Test MER.2. 
WVU.1.1

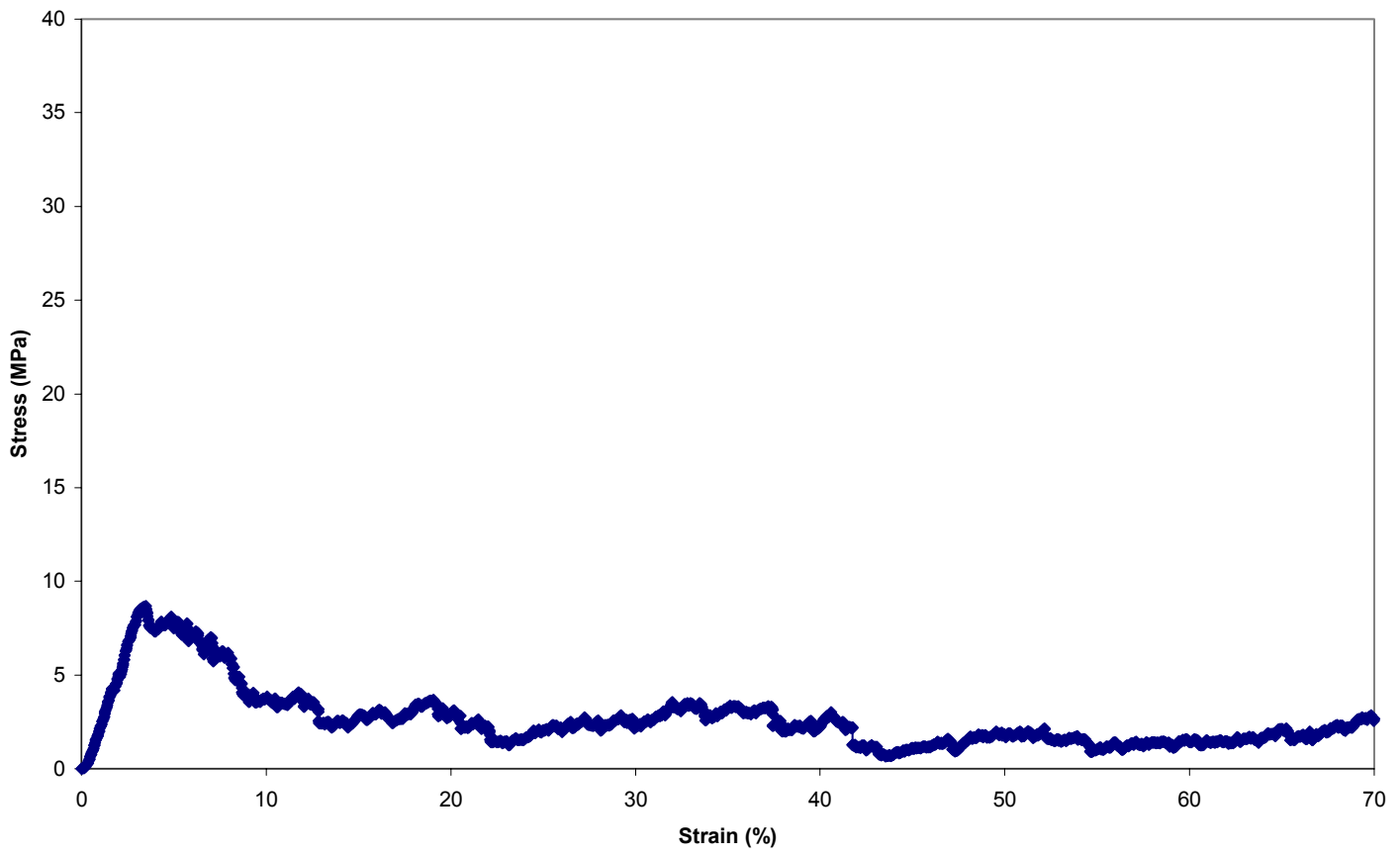

WVU.1.2

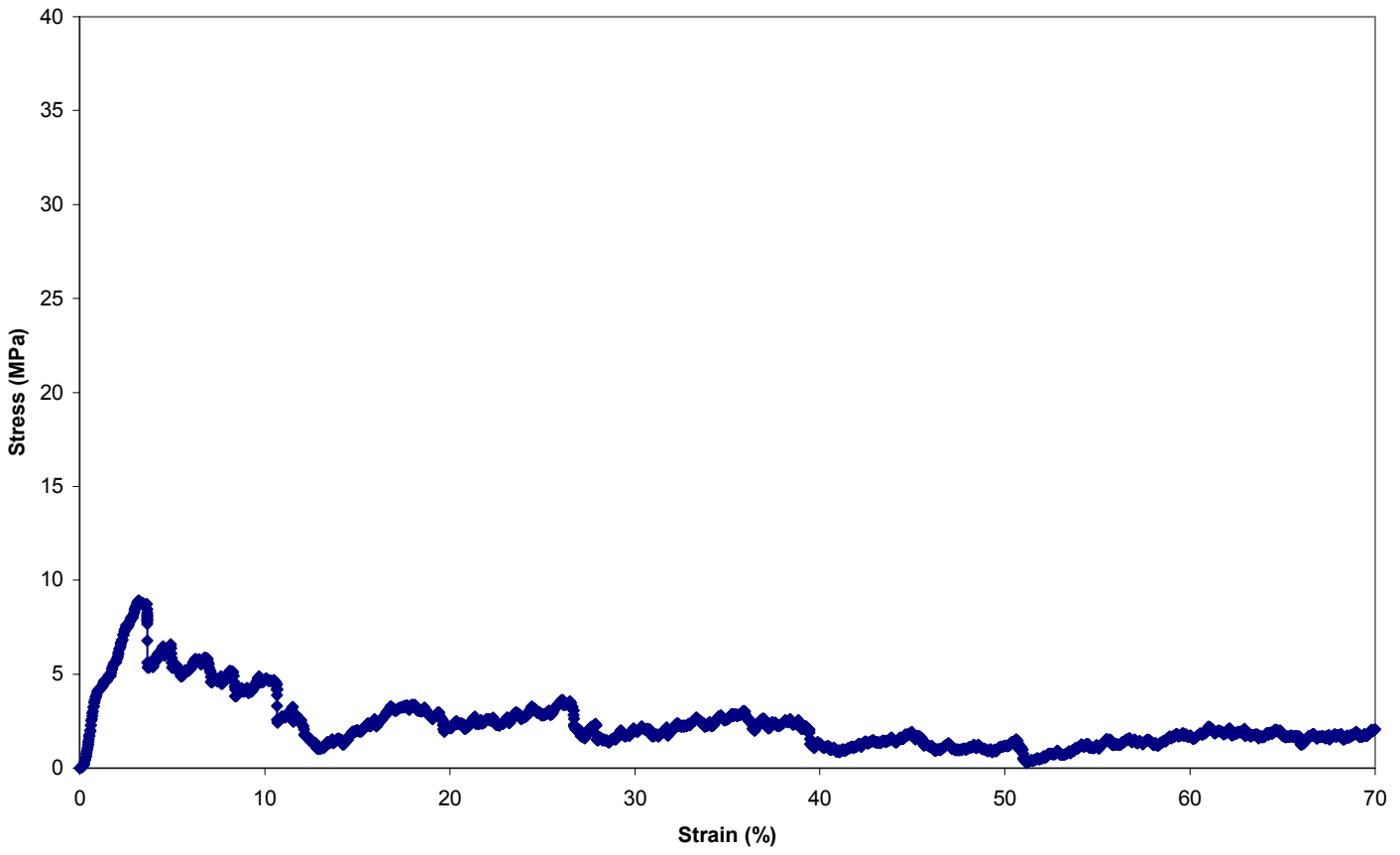

Figure A.59. Stress-Strain Plots for Samples 1 and 2 of Test WVU.1. 
WVU.1.3

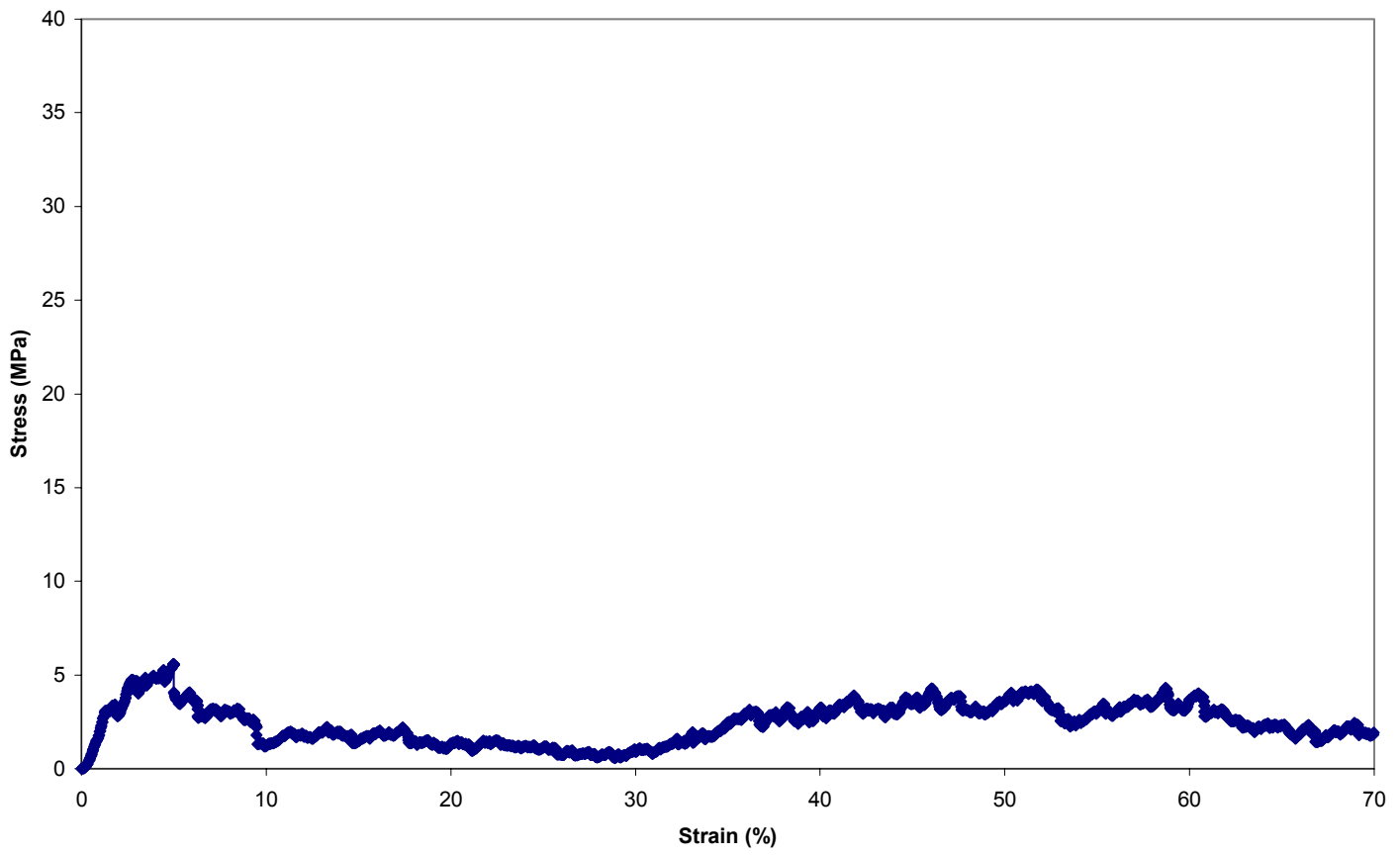

WVU.1.4

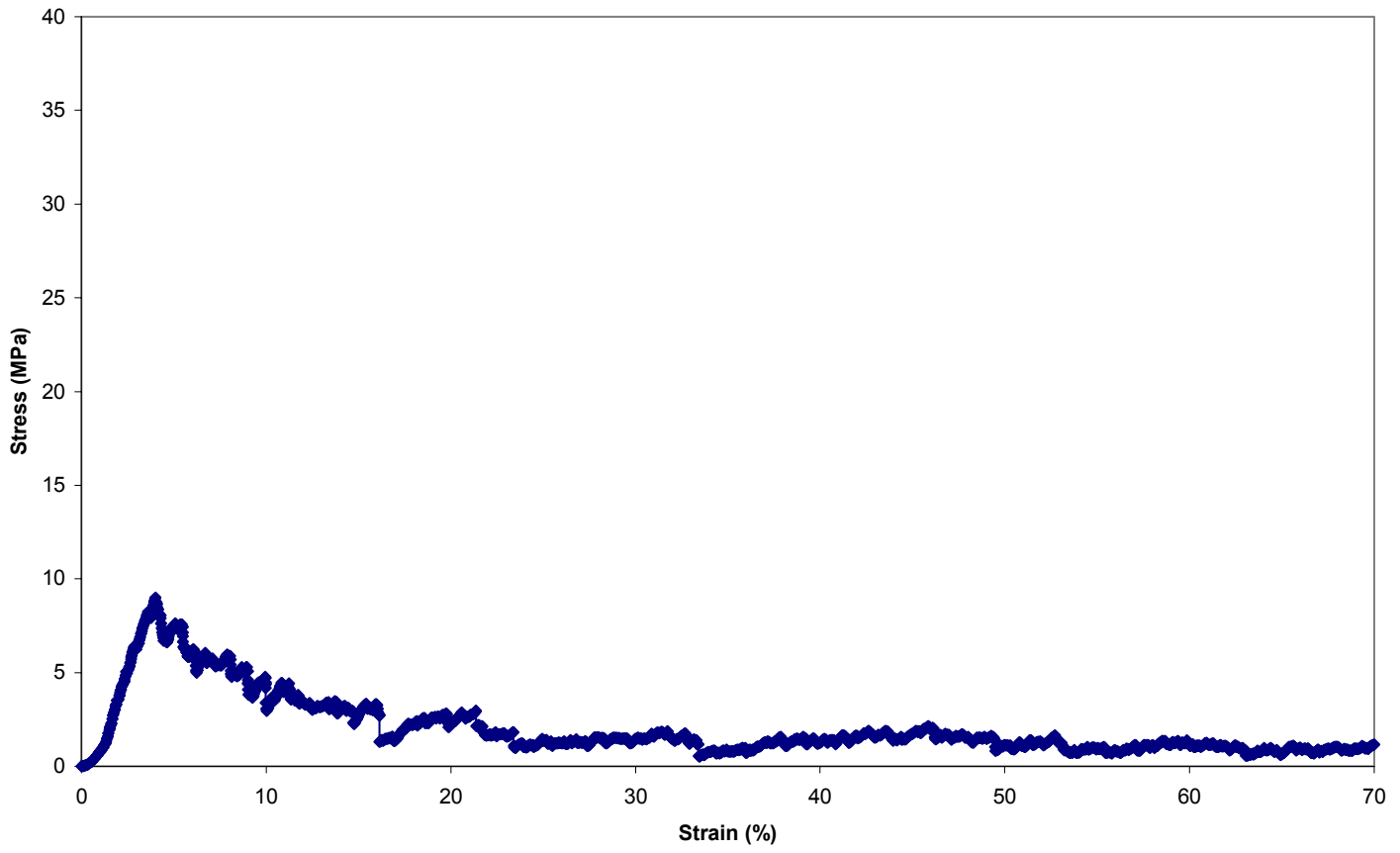

Figure A.60. Stress-Strain Plots for Samples 3 and 4 of Test WVU.1. 
WVU.1.5

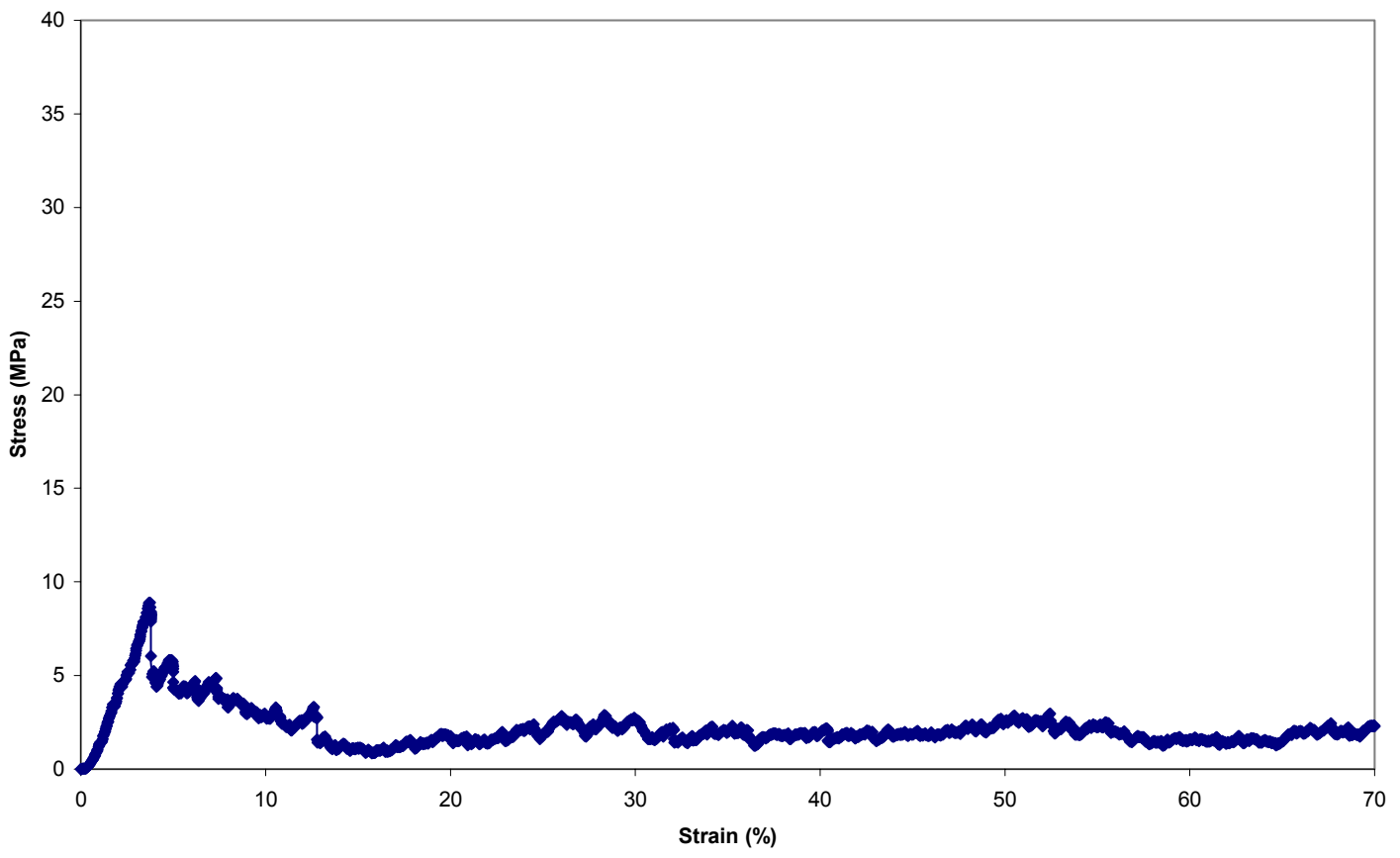

WVU.2.6

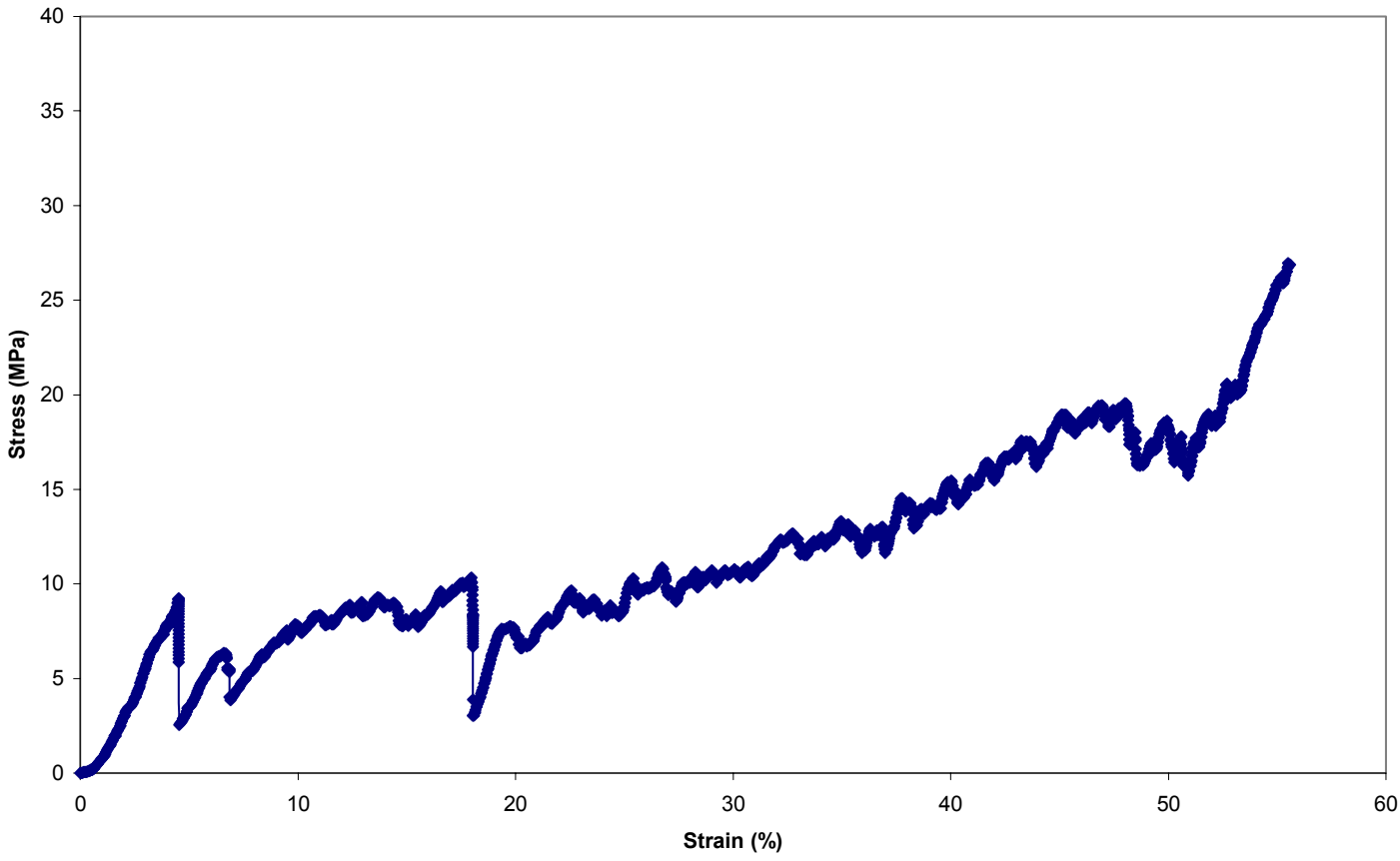

Figure A.61. Stress-Strain Plots for Sample 5 of Test WVU.1 and Sample 6 of Test WVU.2. 
WVU.2.7

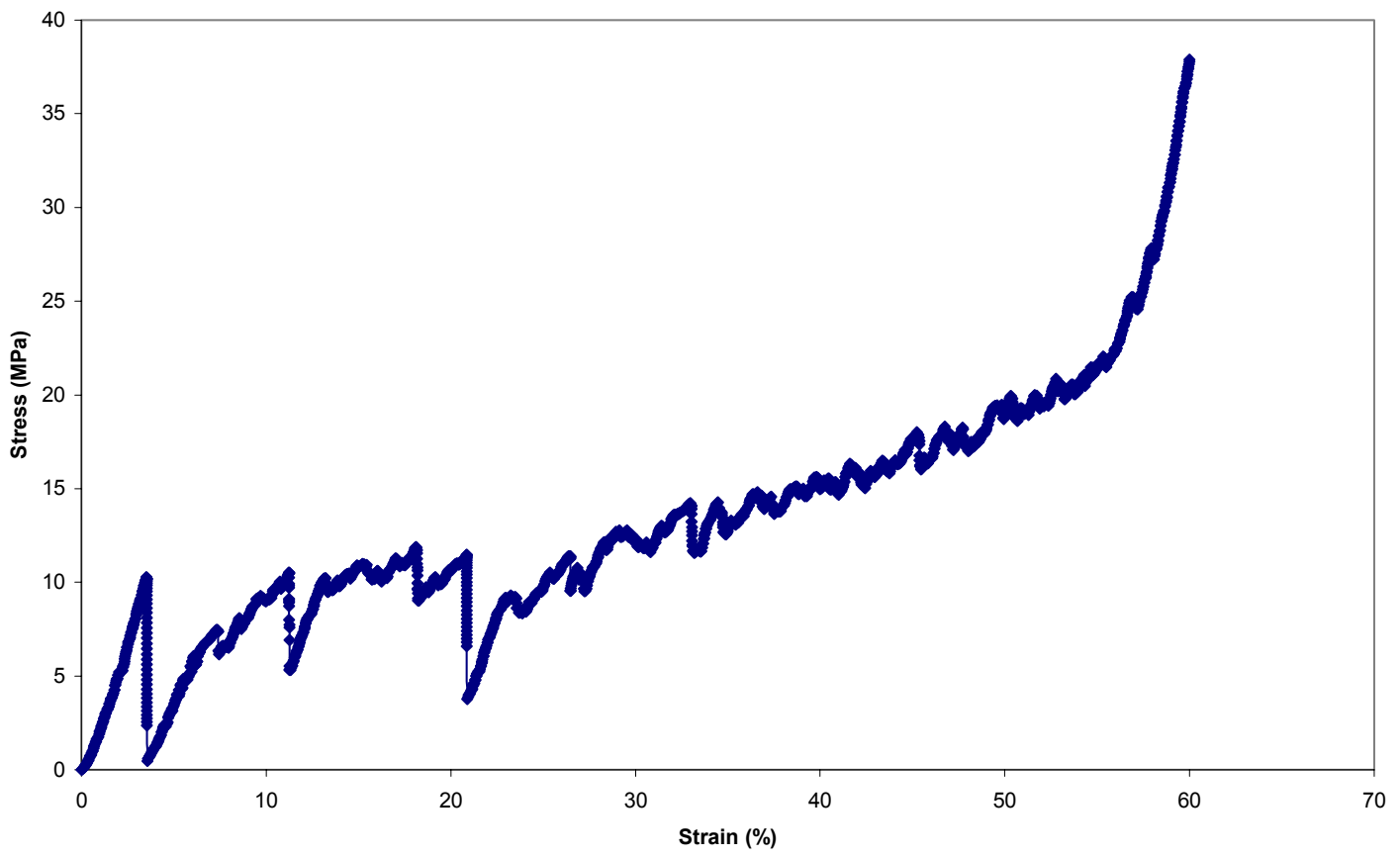

WVU.2.8

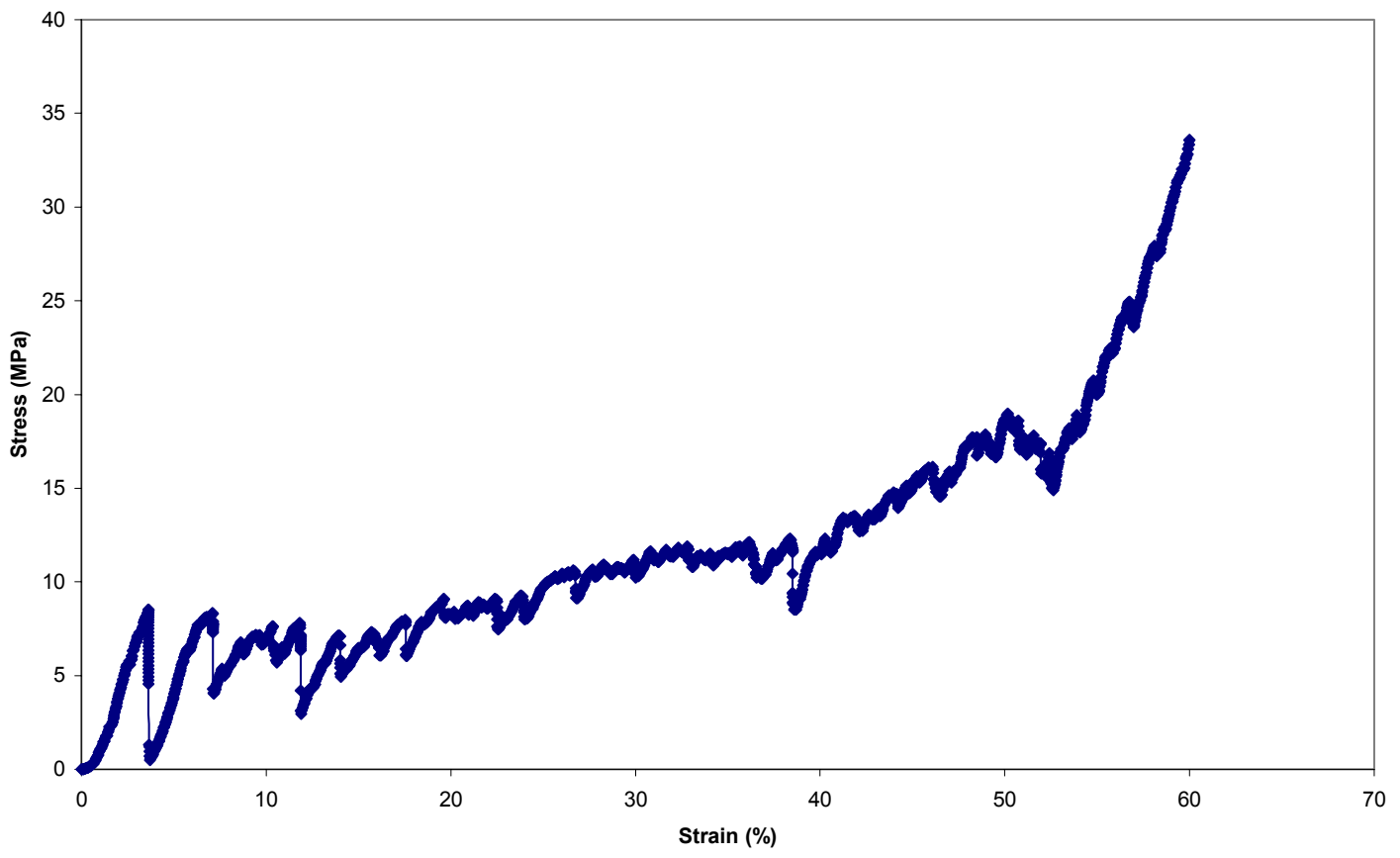

Figure A.62. Stress-Strain Plots for Samples 7 and 8 of Test WVU.2. 
WVU.2.9

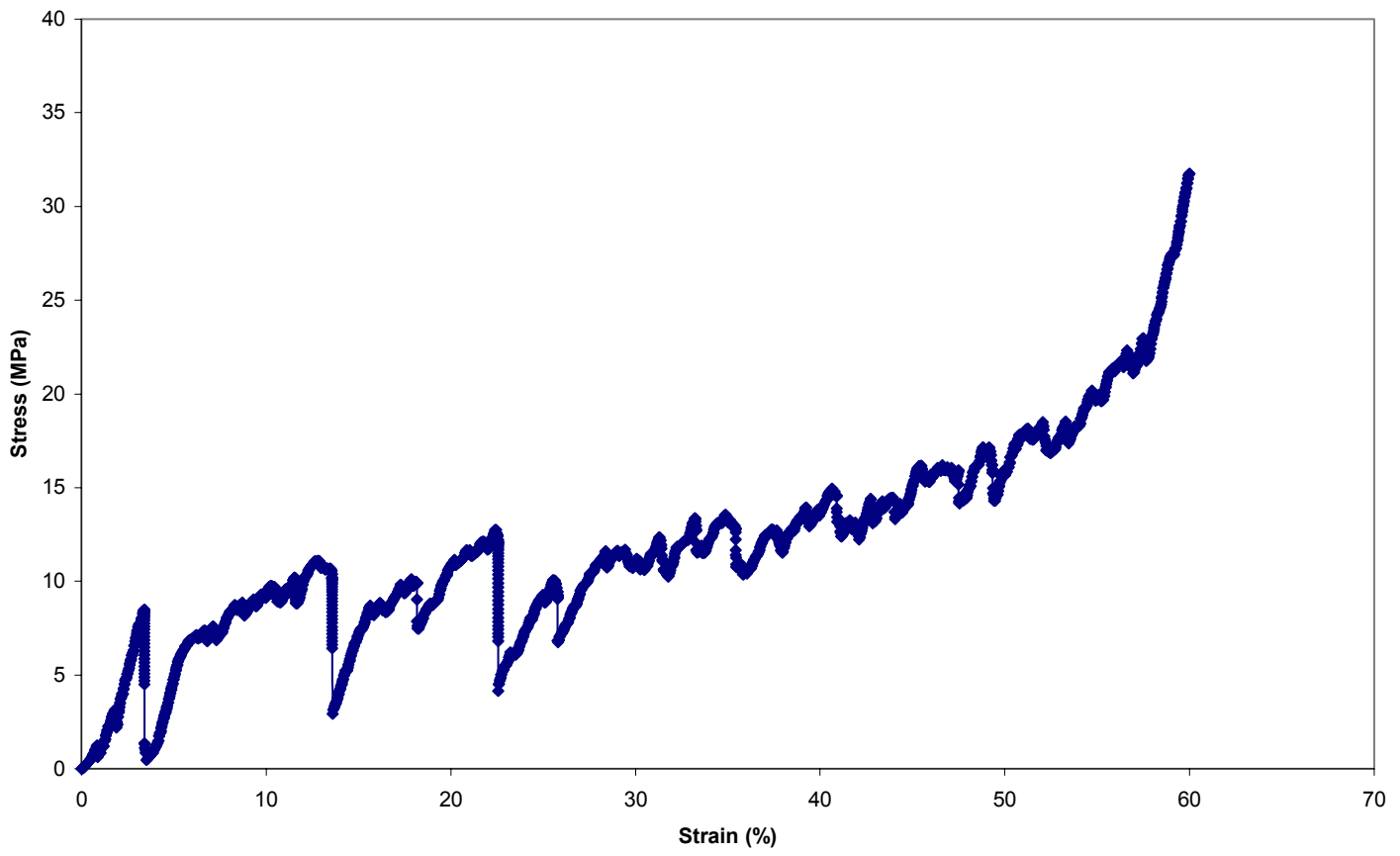

WVU.2.10

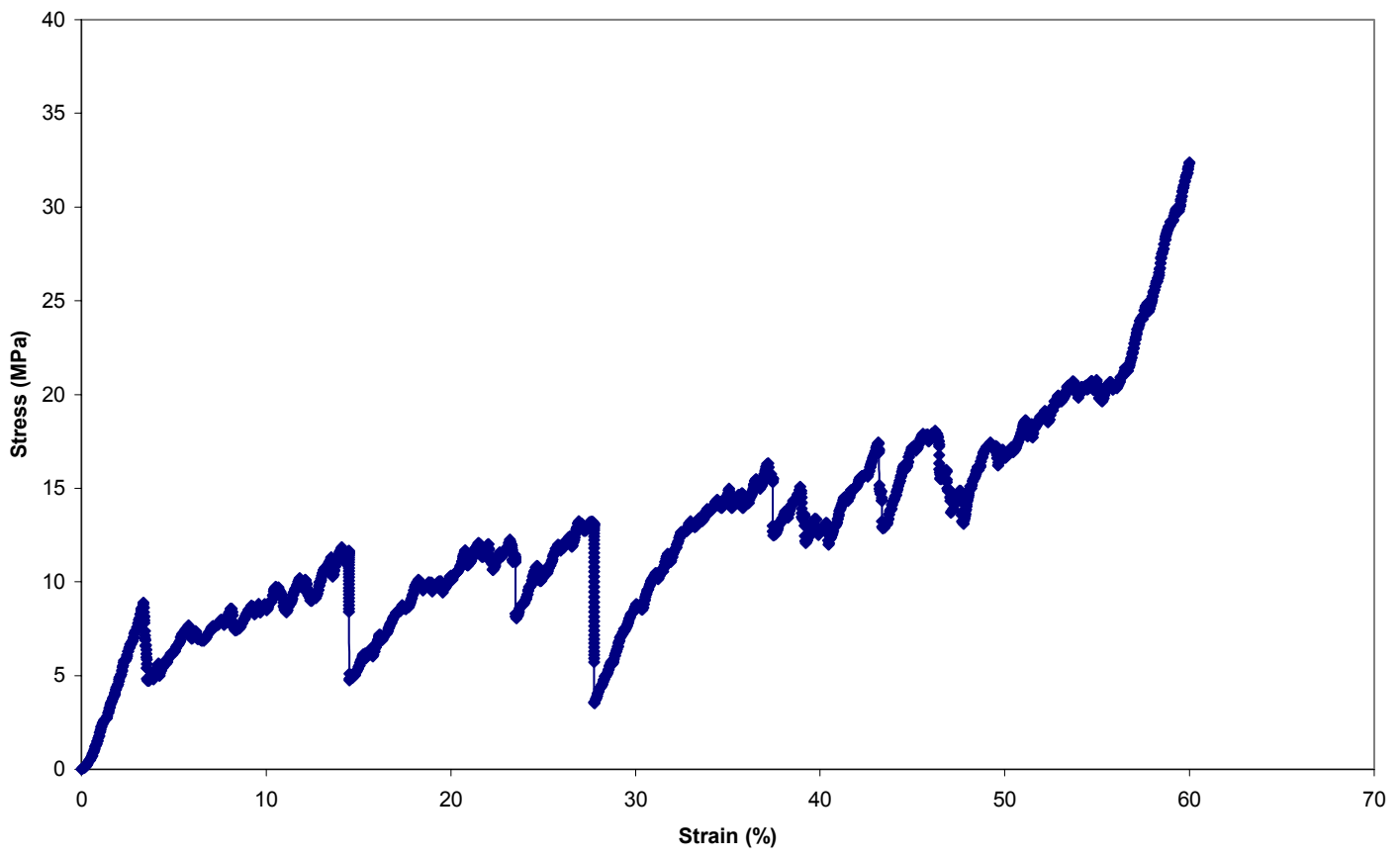

Figure A.63. Stress-Strain Plots for Samples 9 and 10 of Test WVU.2. 


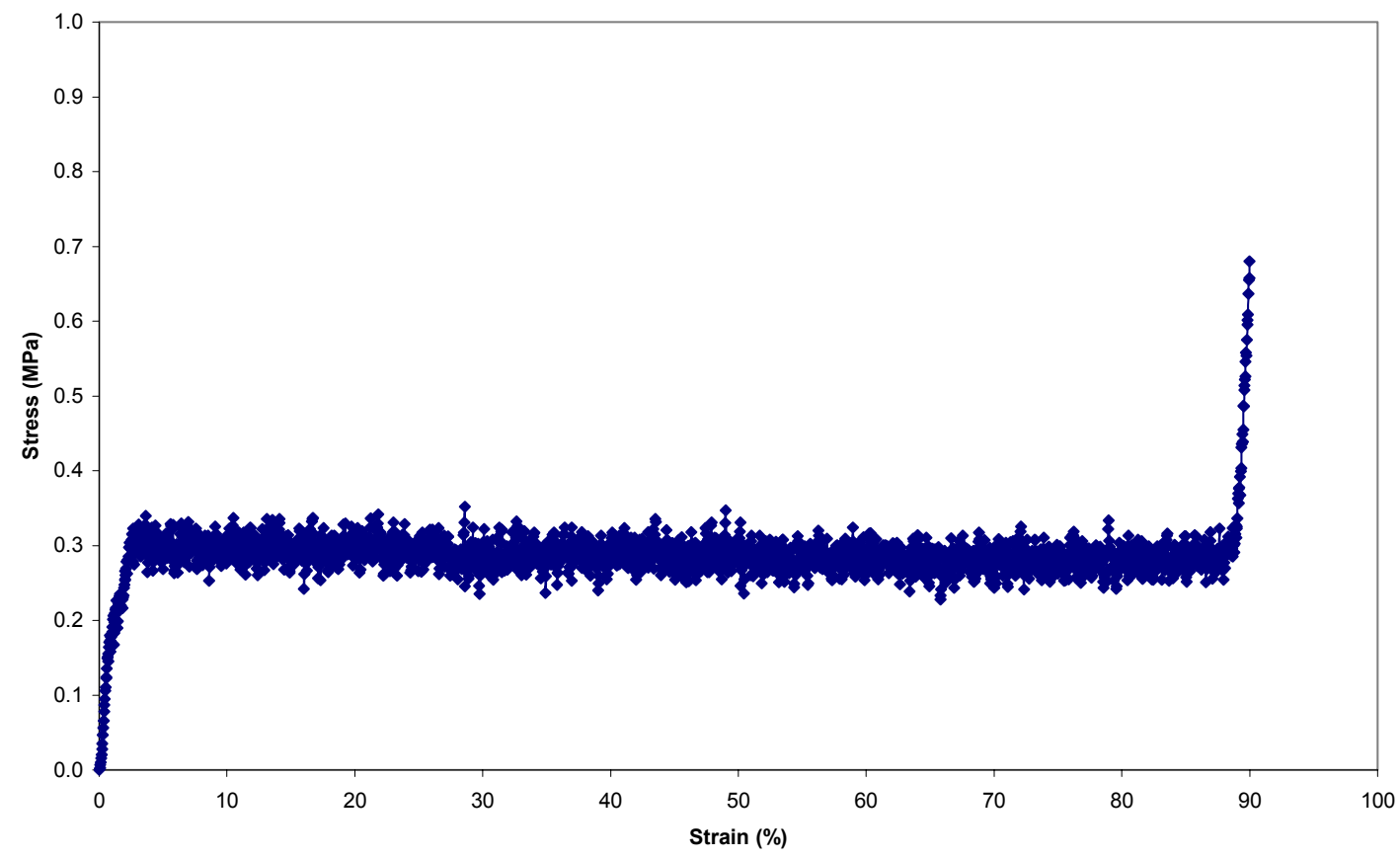

U.1.2

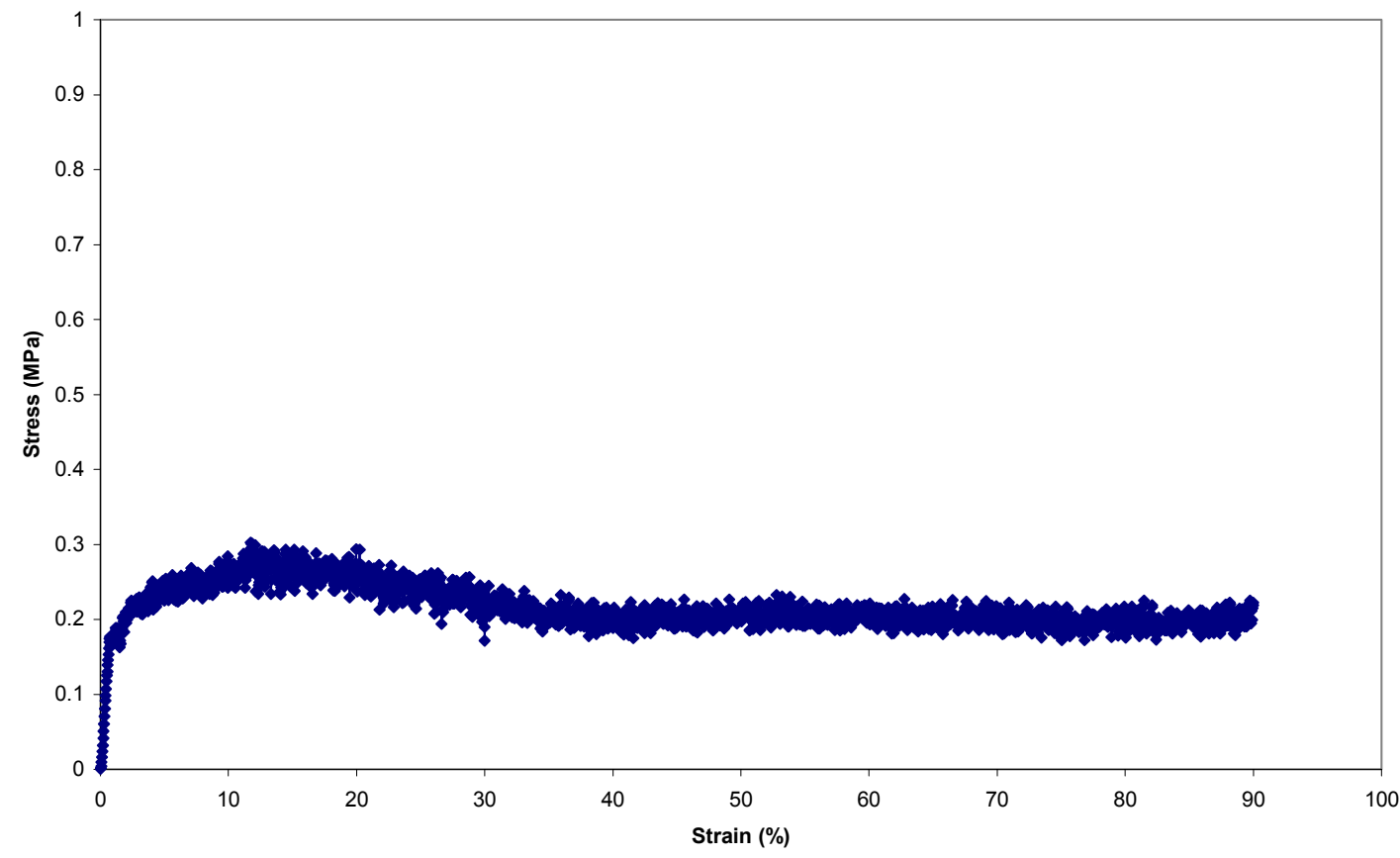

Figure A.64. Stress-Strain Plots for Samples 1 and 2 of Test U.1. 


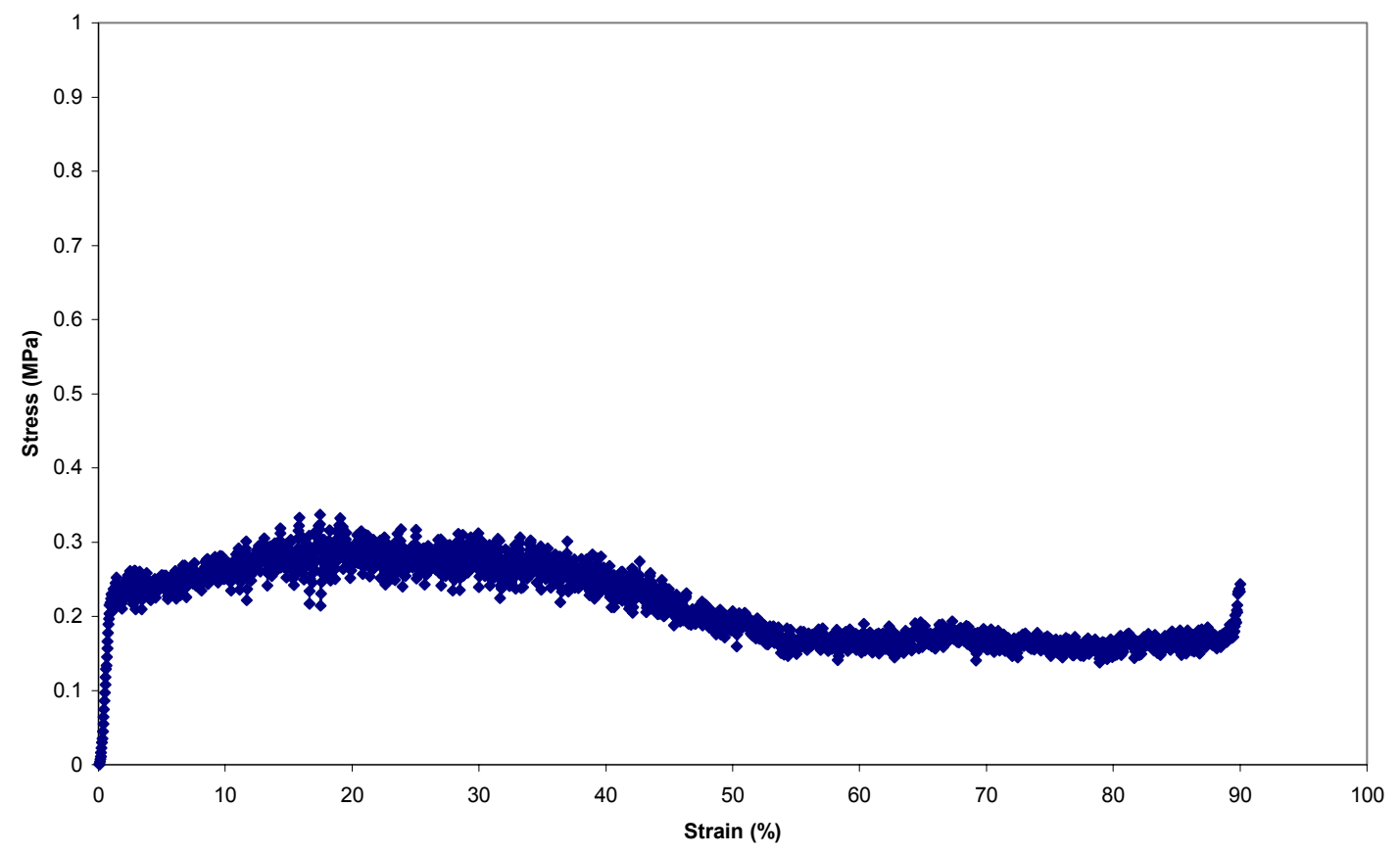

U.1.4

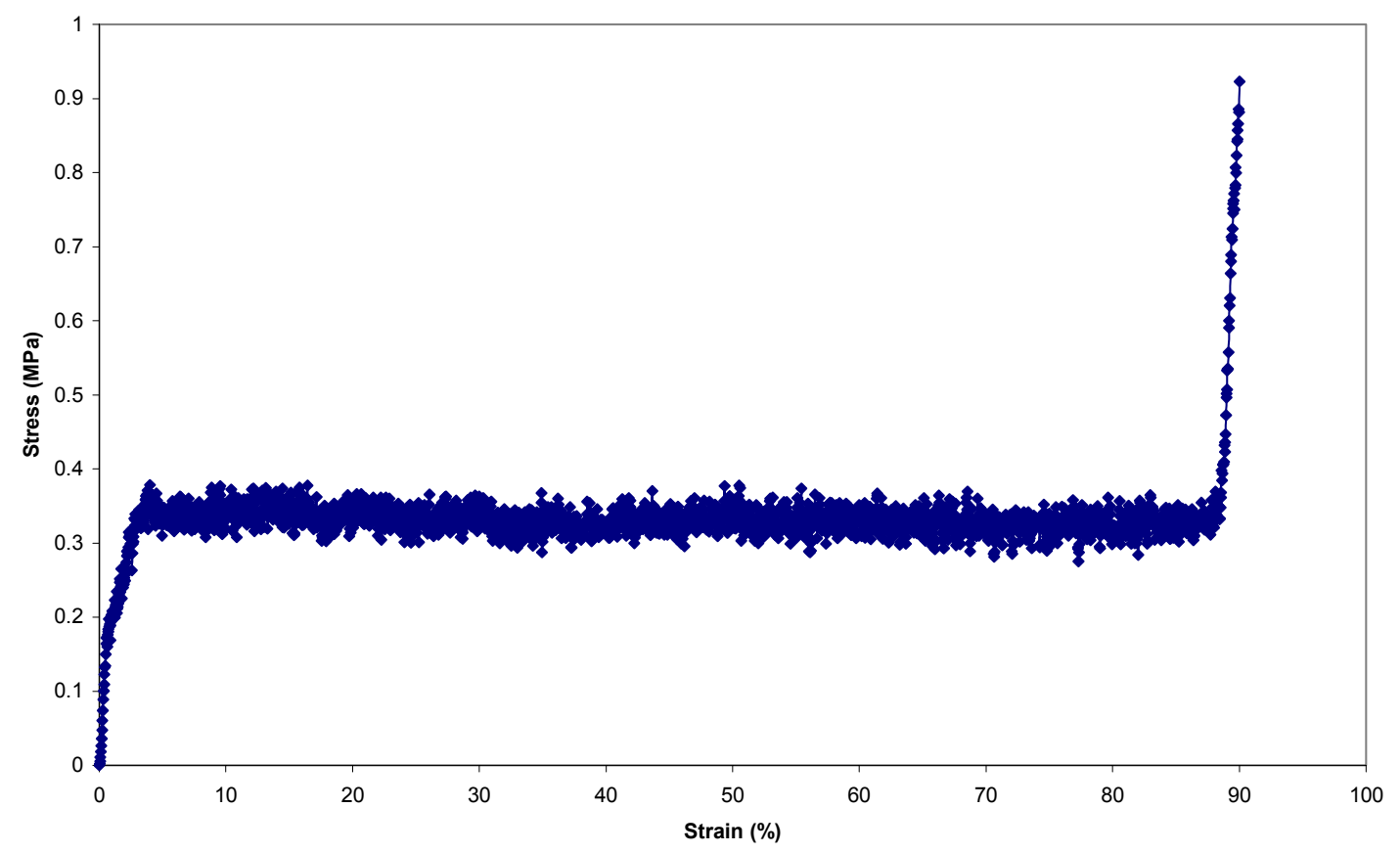

Figure A.65. Stress-Strain Plots for Samples 3 and 4 of Test U.1. 


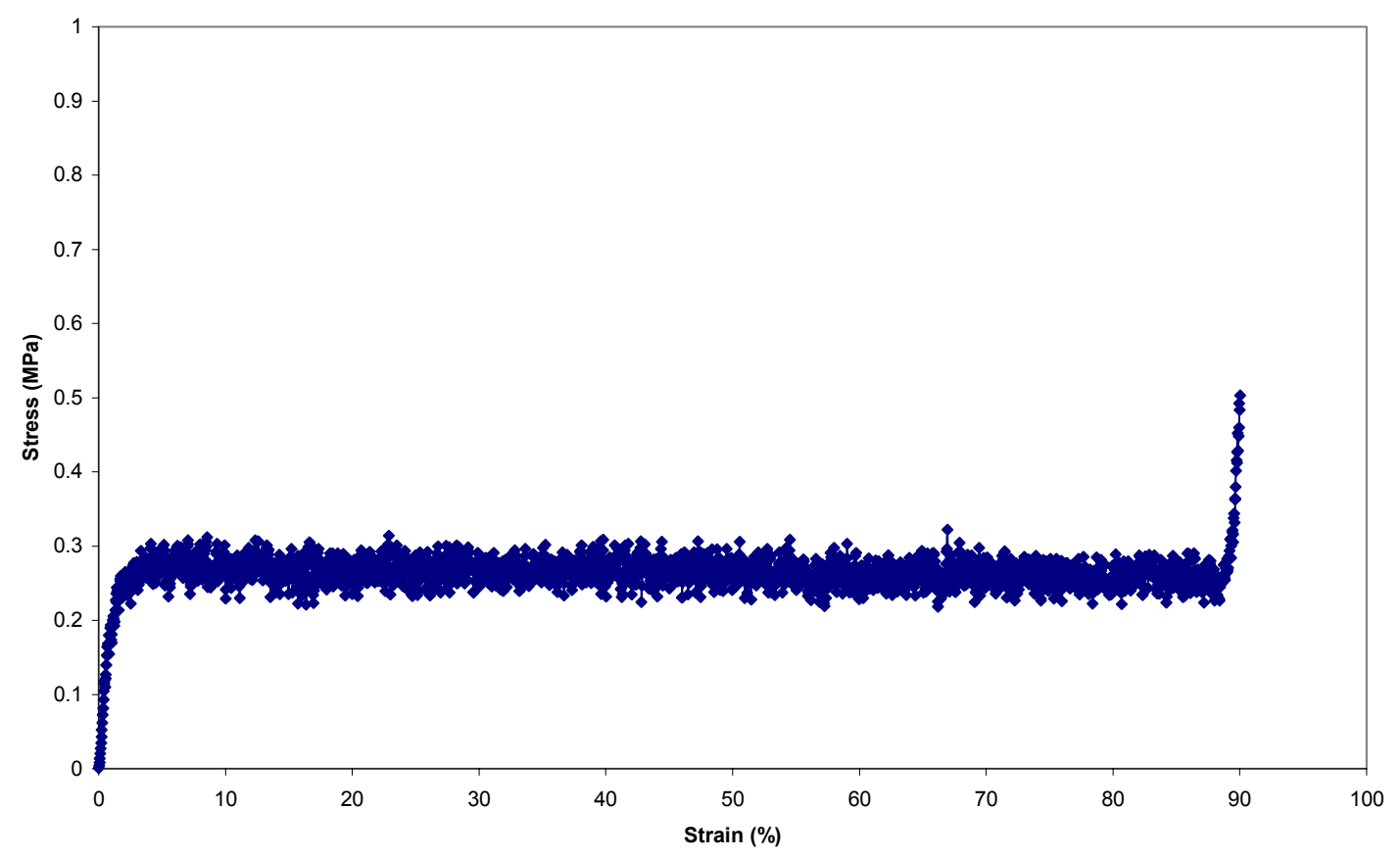

U.2.1

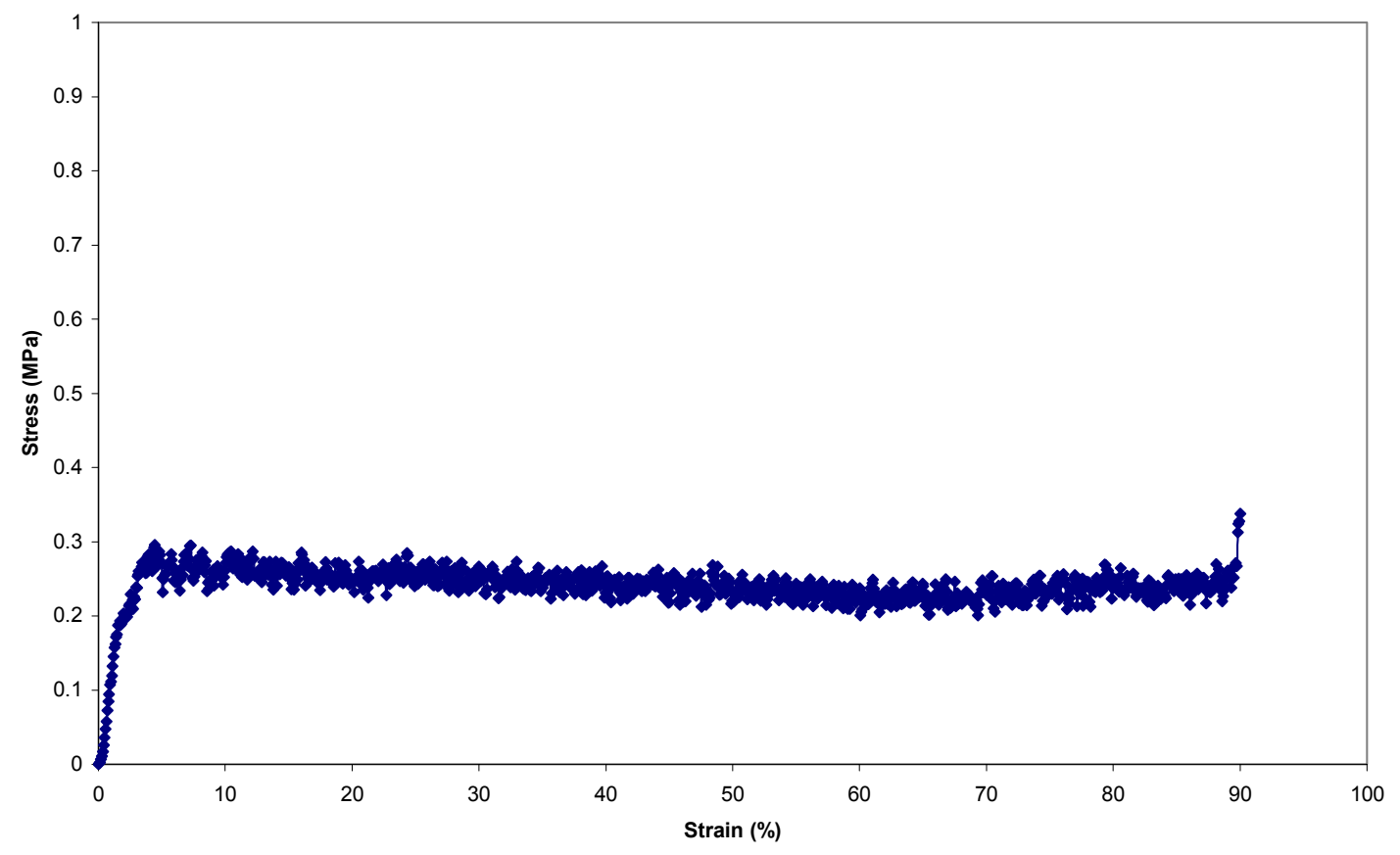

Figure A.66. Stress-Strain Plots for Sample 5 of Test U.1 and Sample 1 of Test U.2. 


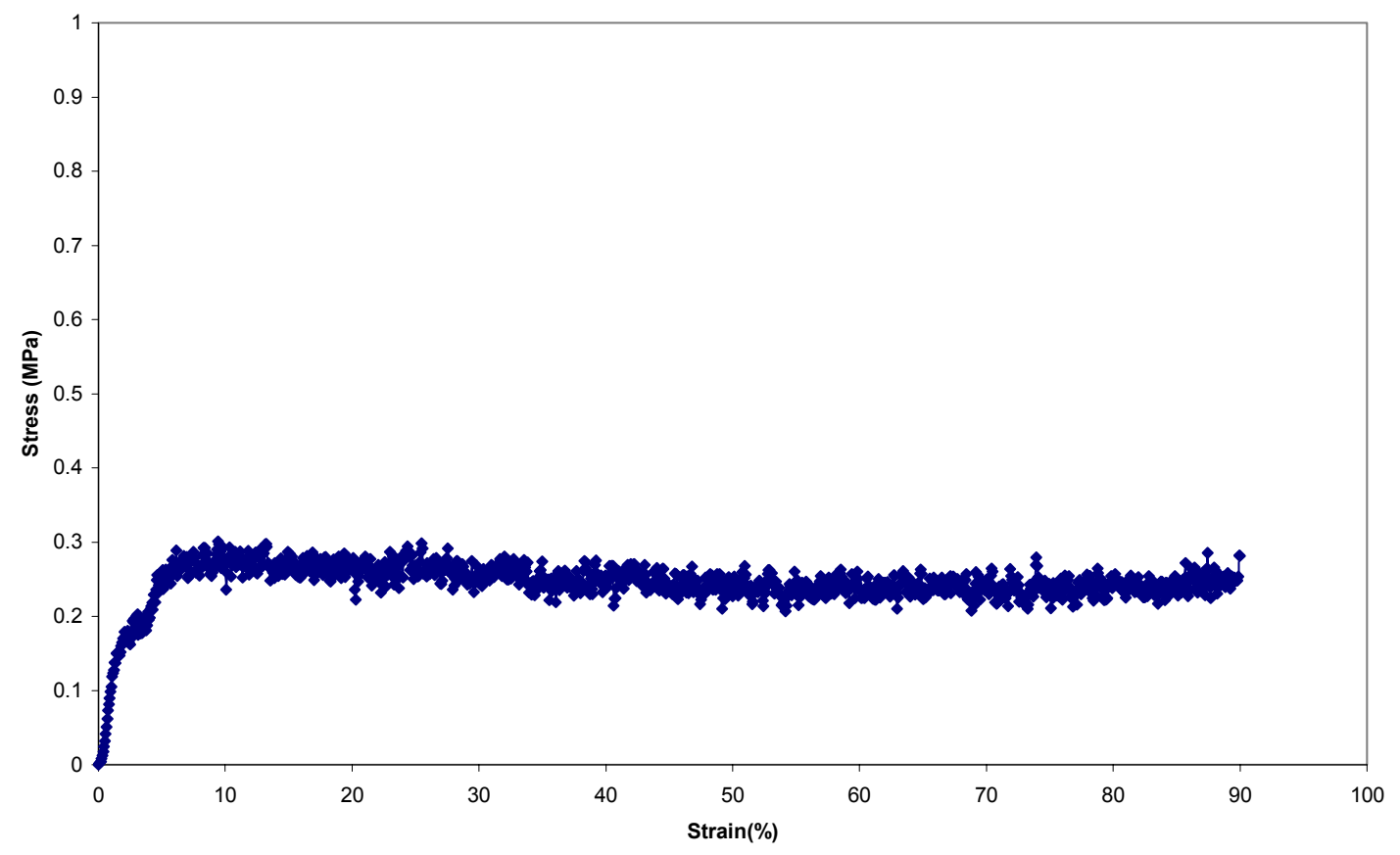

U.2.3

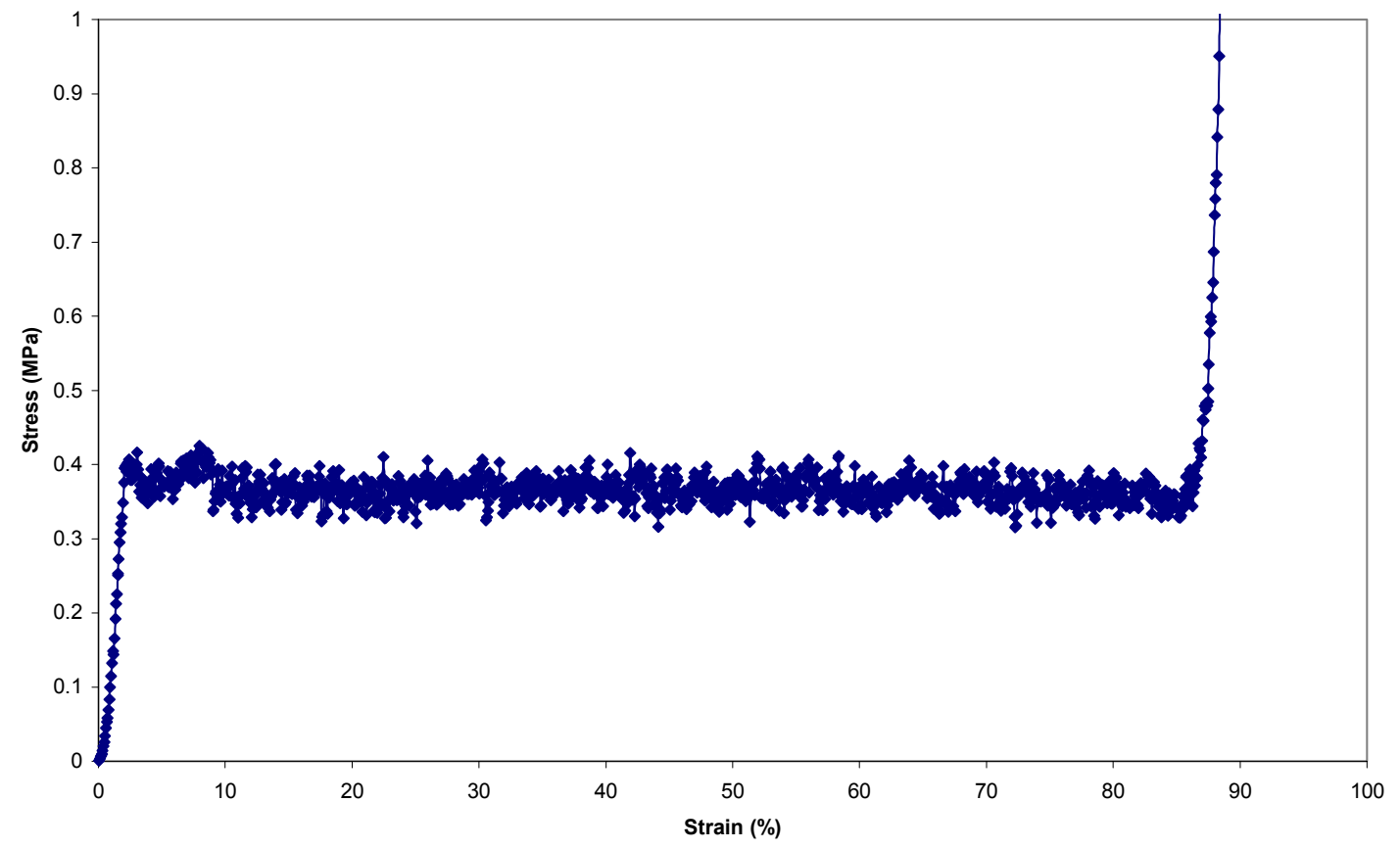

Figure A.67. Stress-Strain Plots for Samples 2 and 3 of Test U.2. 


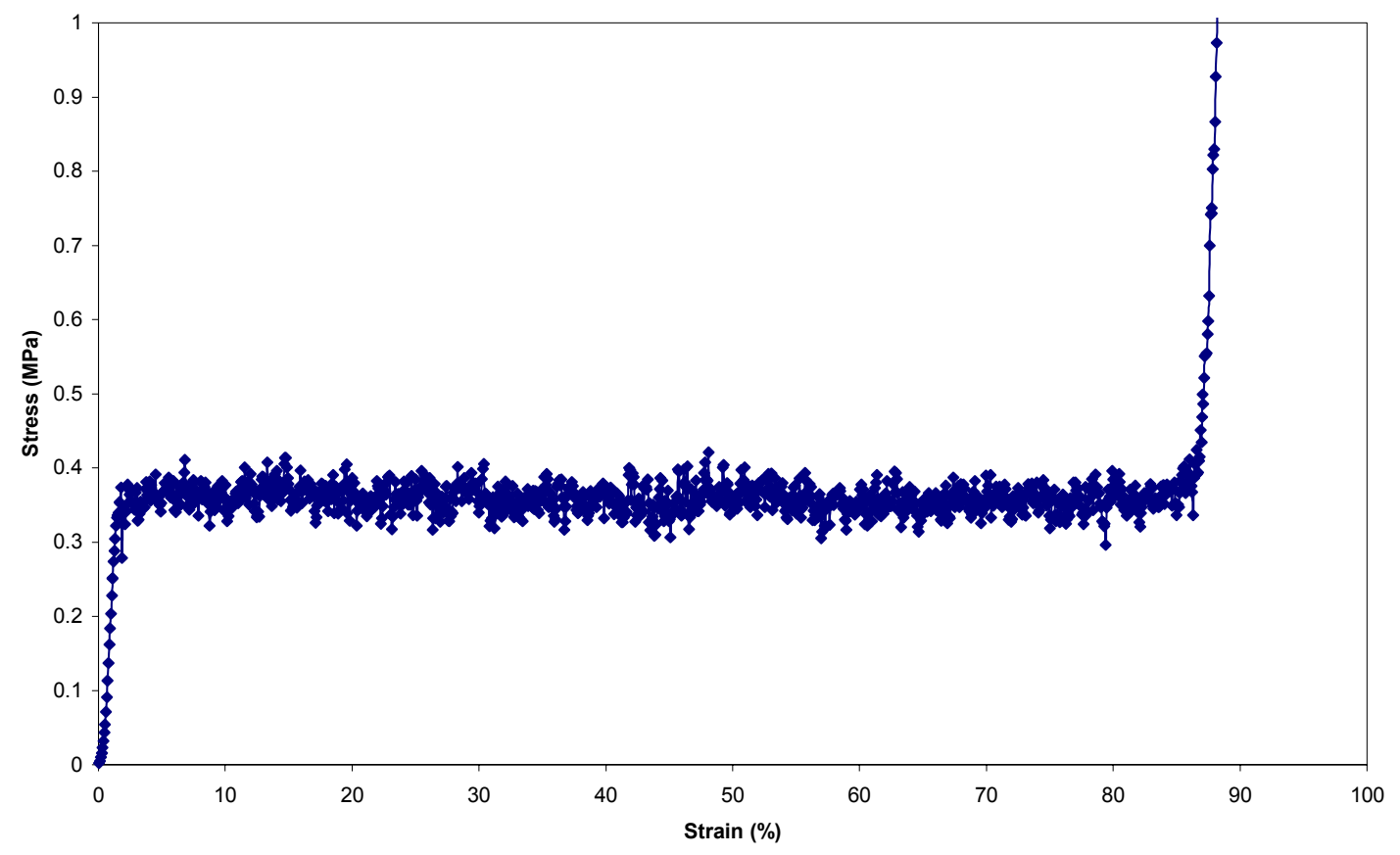

U.3.6

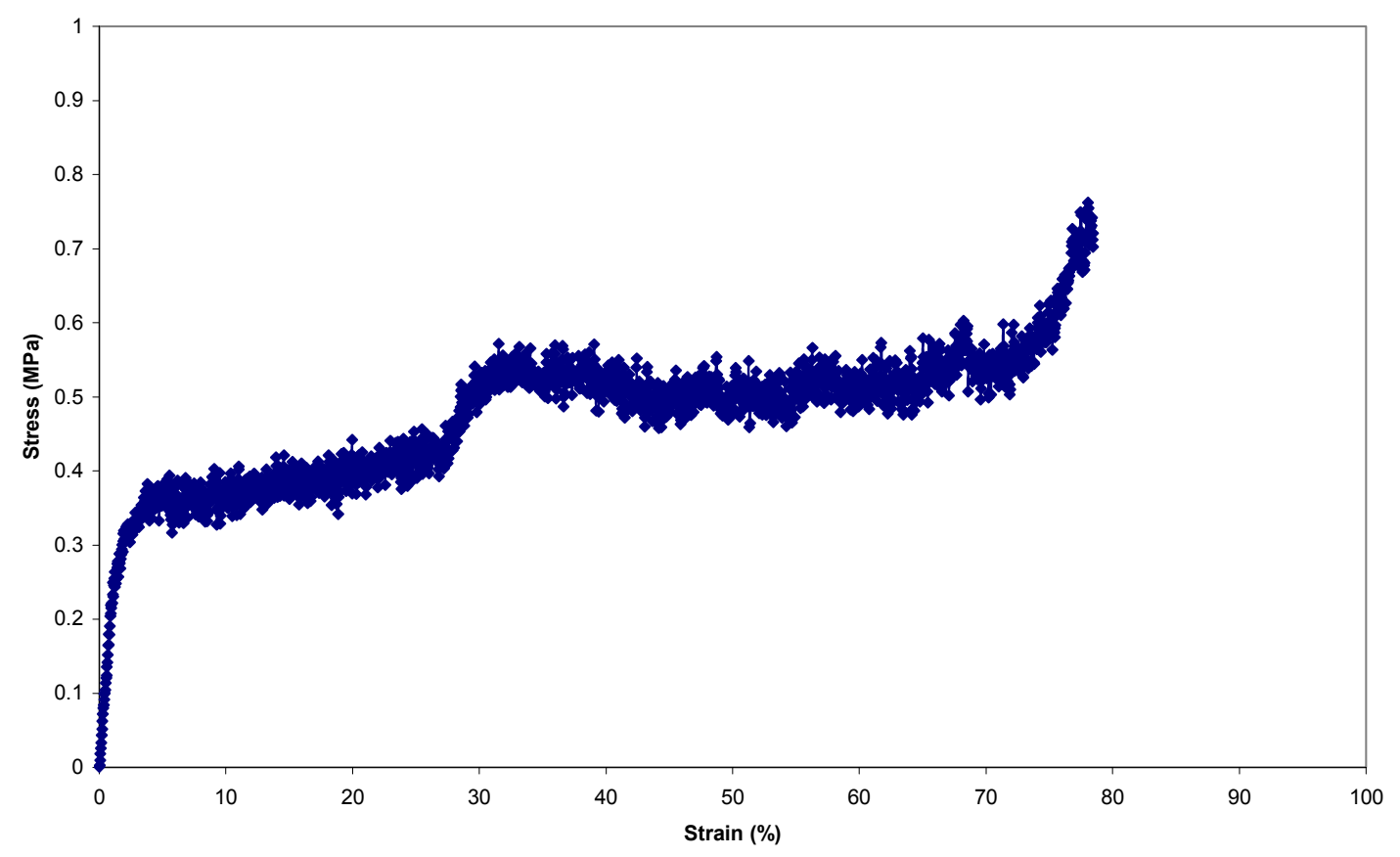

Figure A.68. Stress-Strain Plots for Sample 4 of Test U.2 and Sample 6 of Test U.3. 


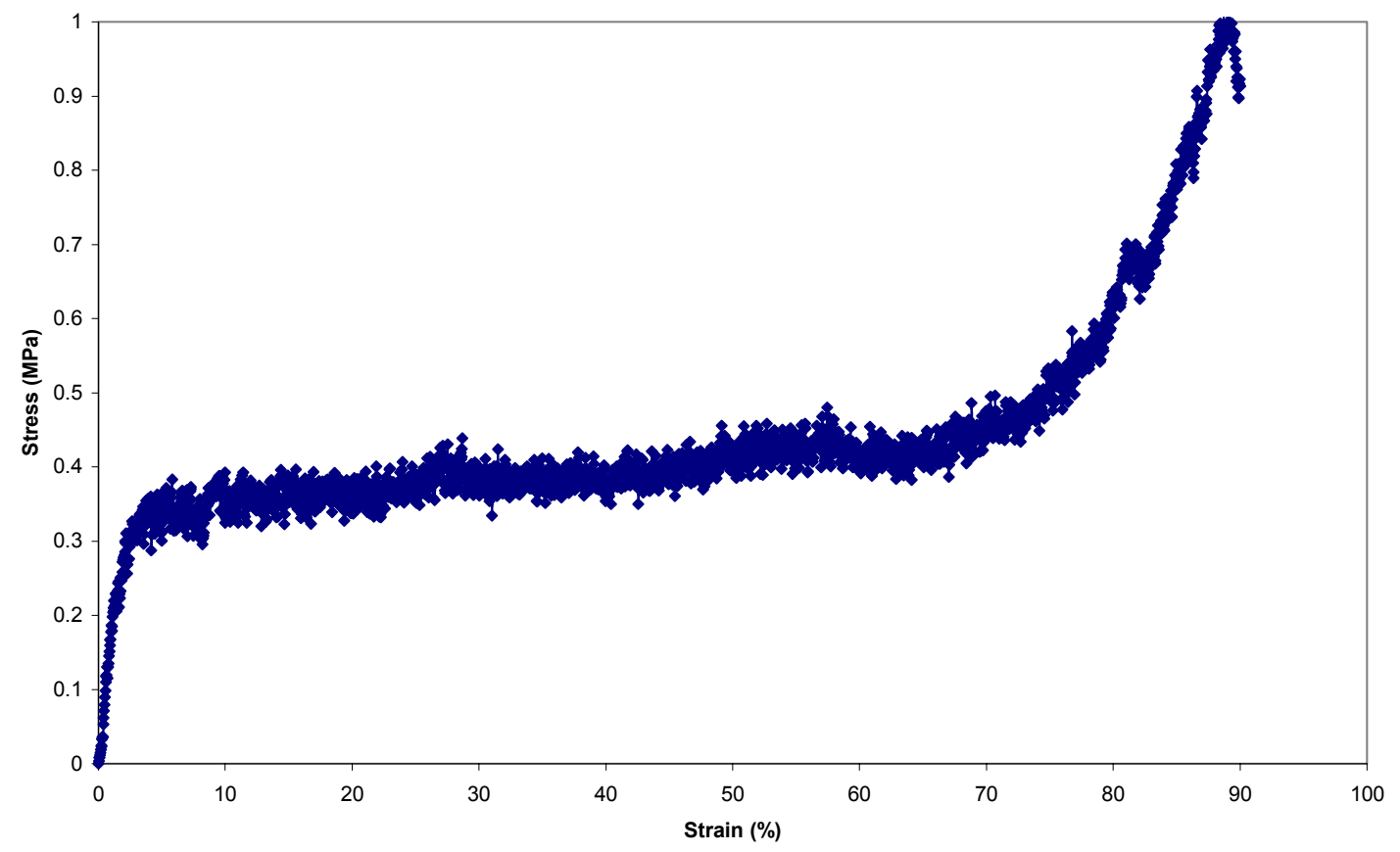

U.3.8

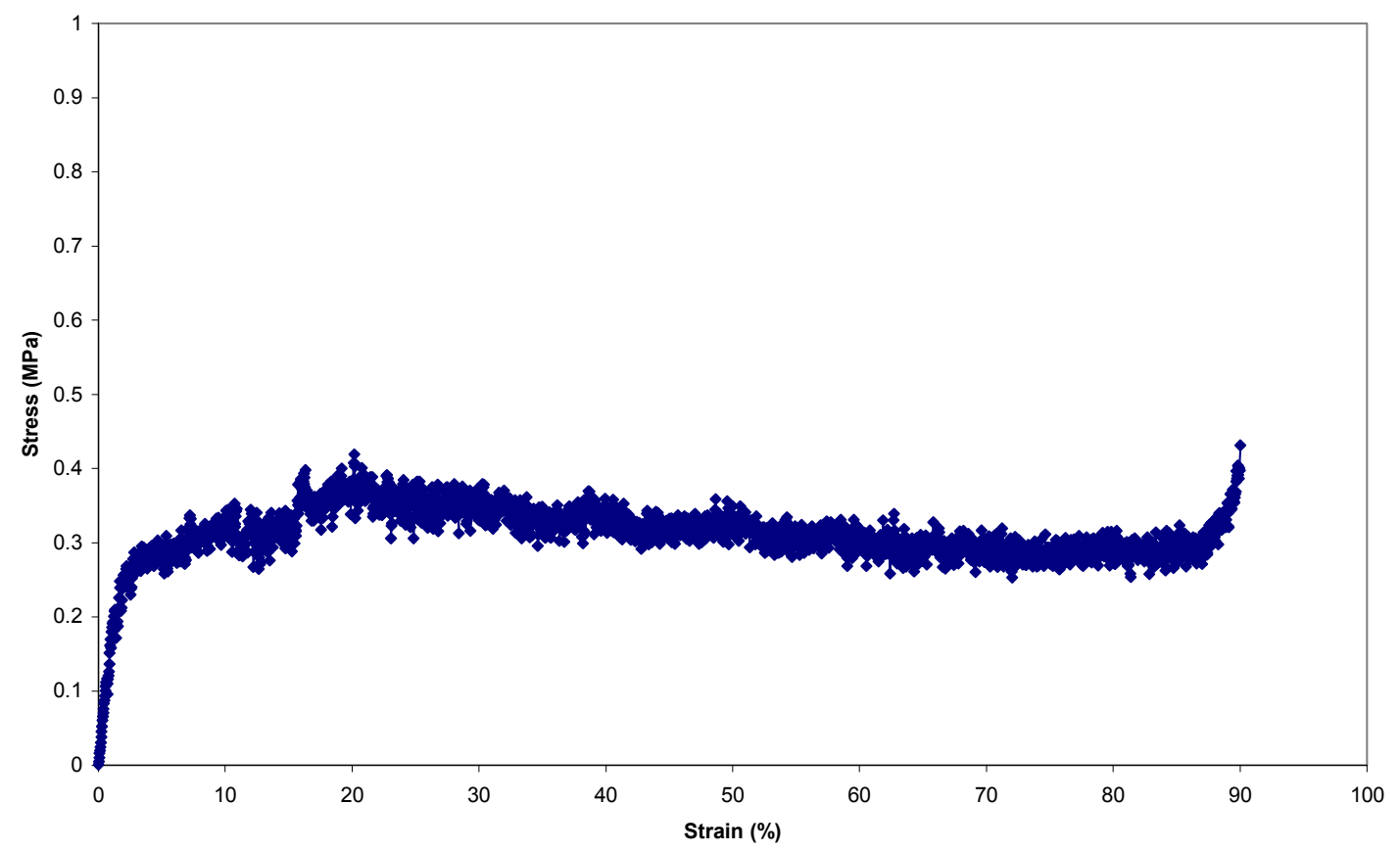

Figure A.69. Stress-Strain Plots for Samples 7 and 8 of Test U.3. 


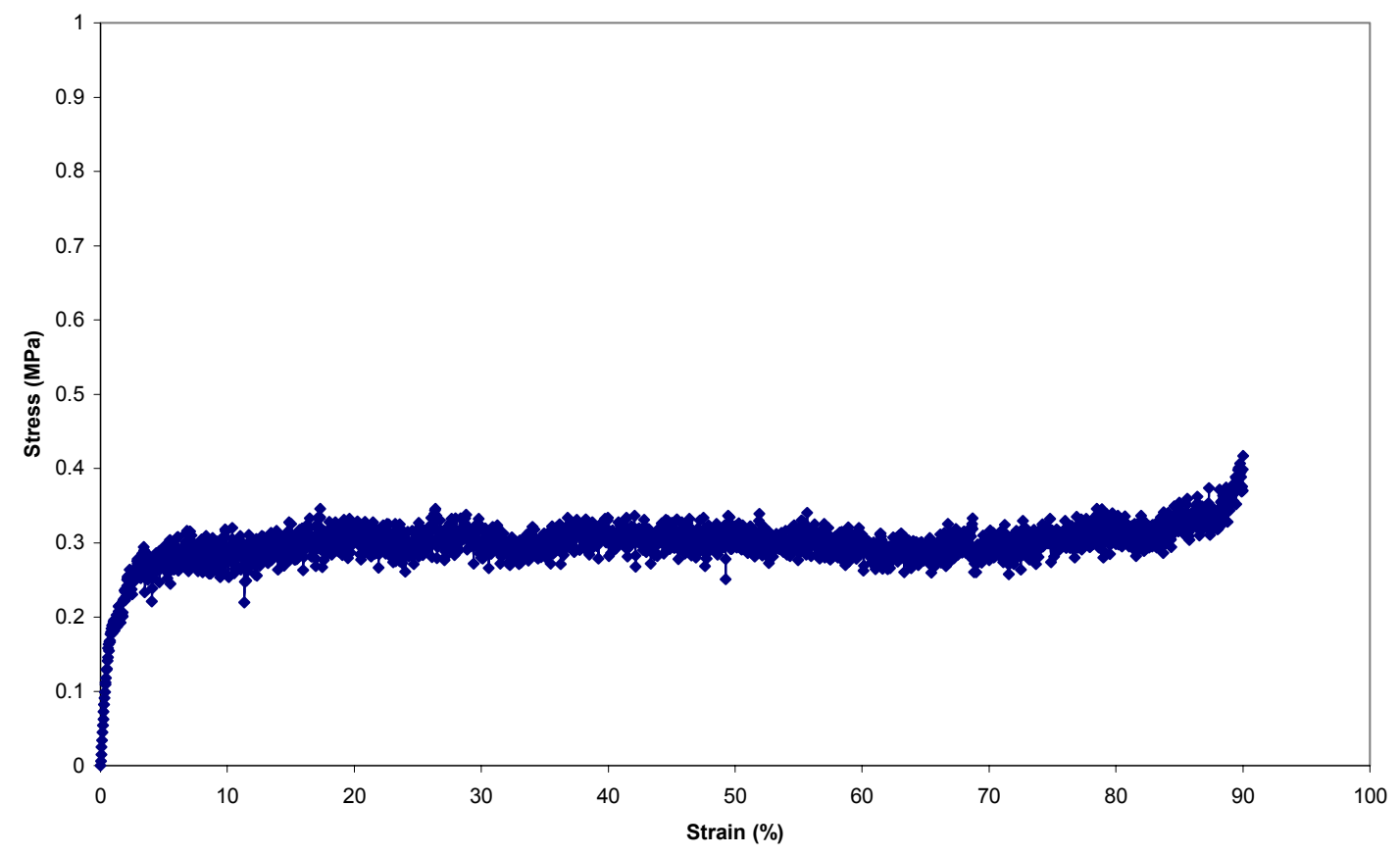

U.3.10

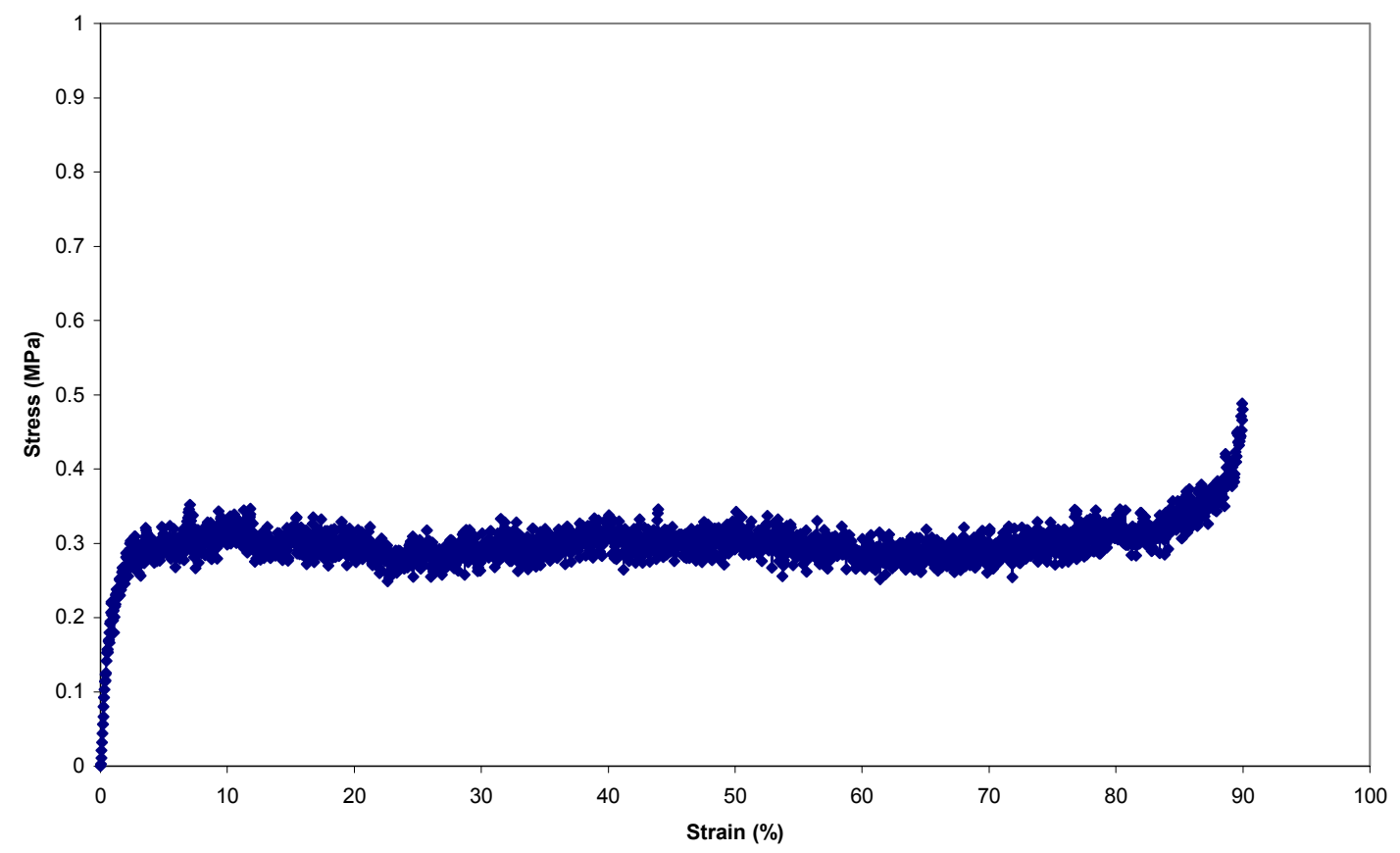

Figure A.70. Stress-Strain Plots for Samples 9 and 10 of Test U.3. 


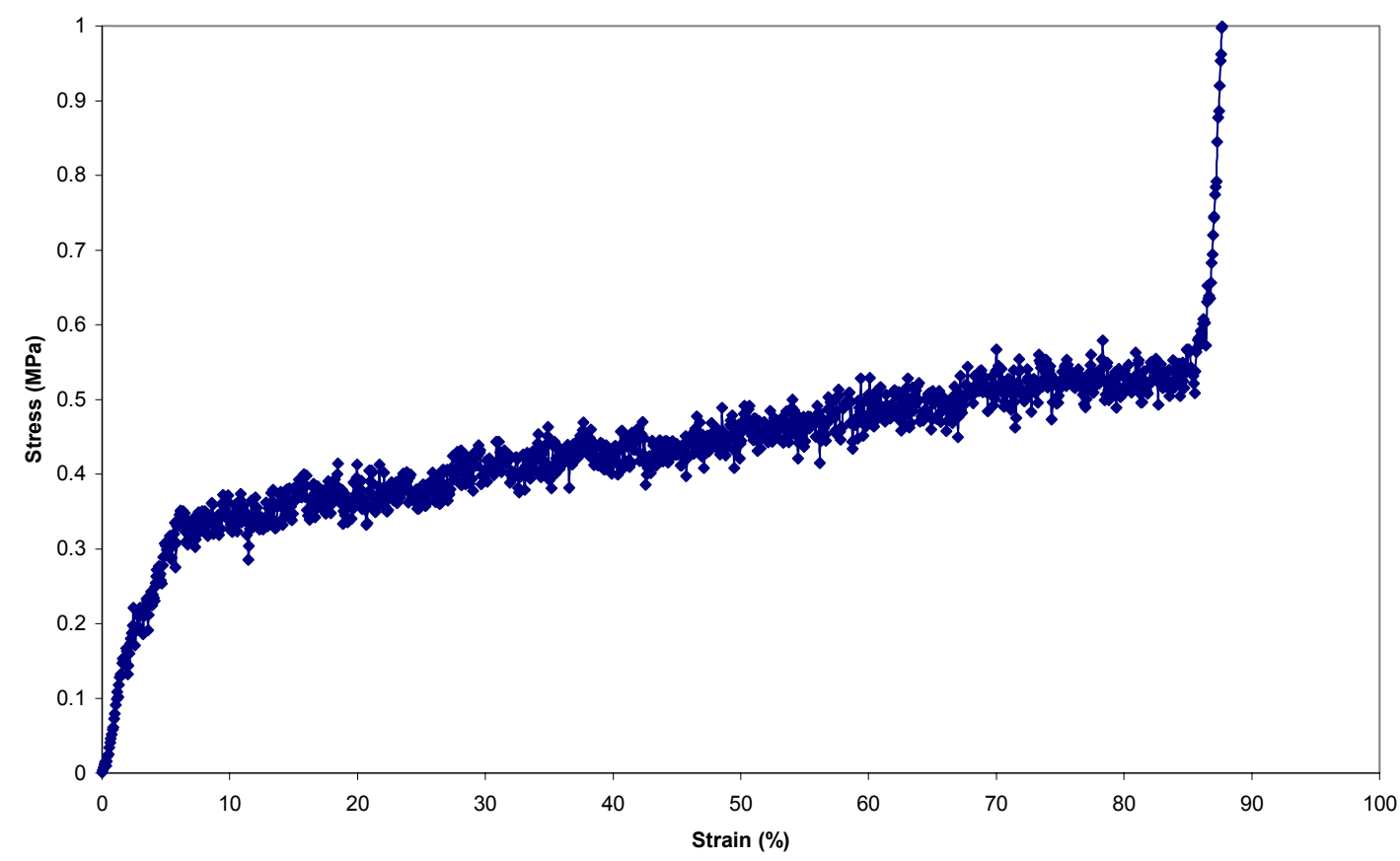

U.4.2

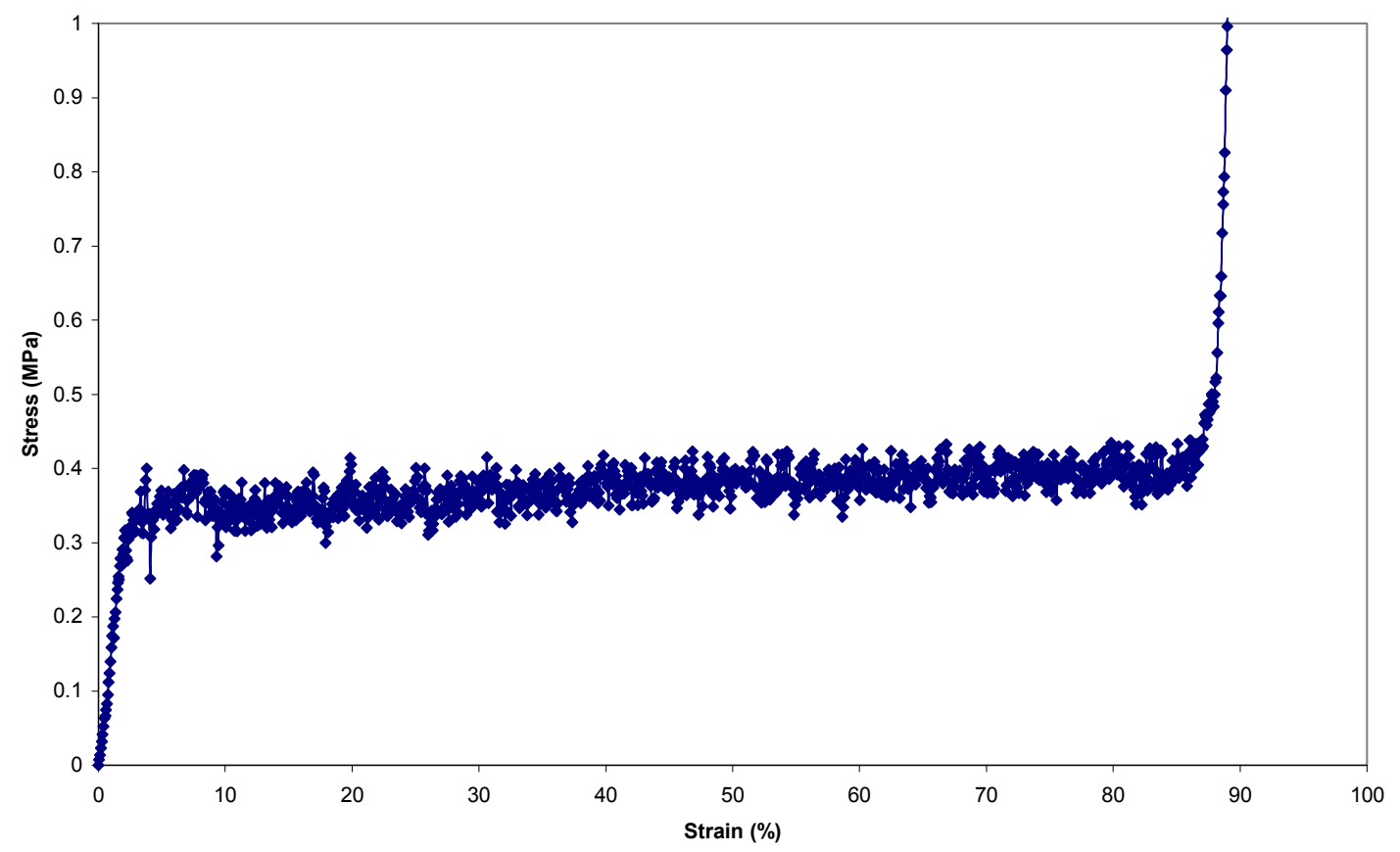

Figure A.71. Stress-Strain Plots for Samples 1 and 2 of Test U.4. 


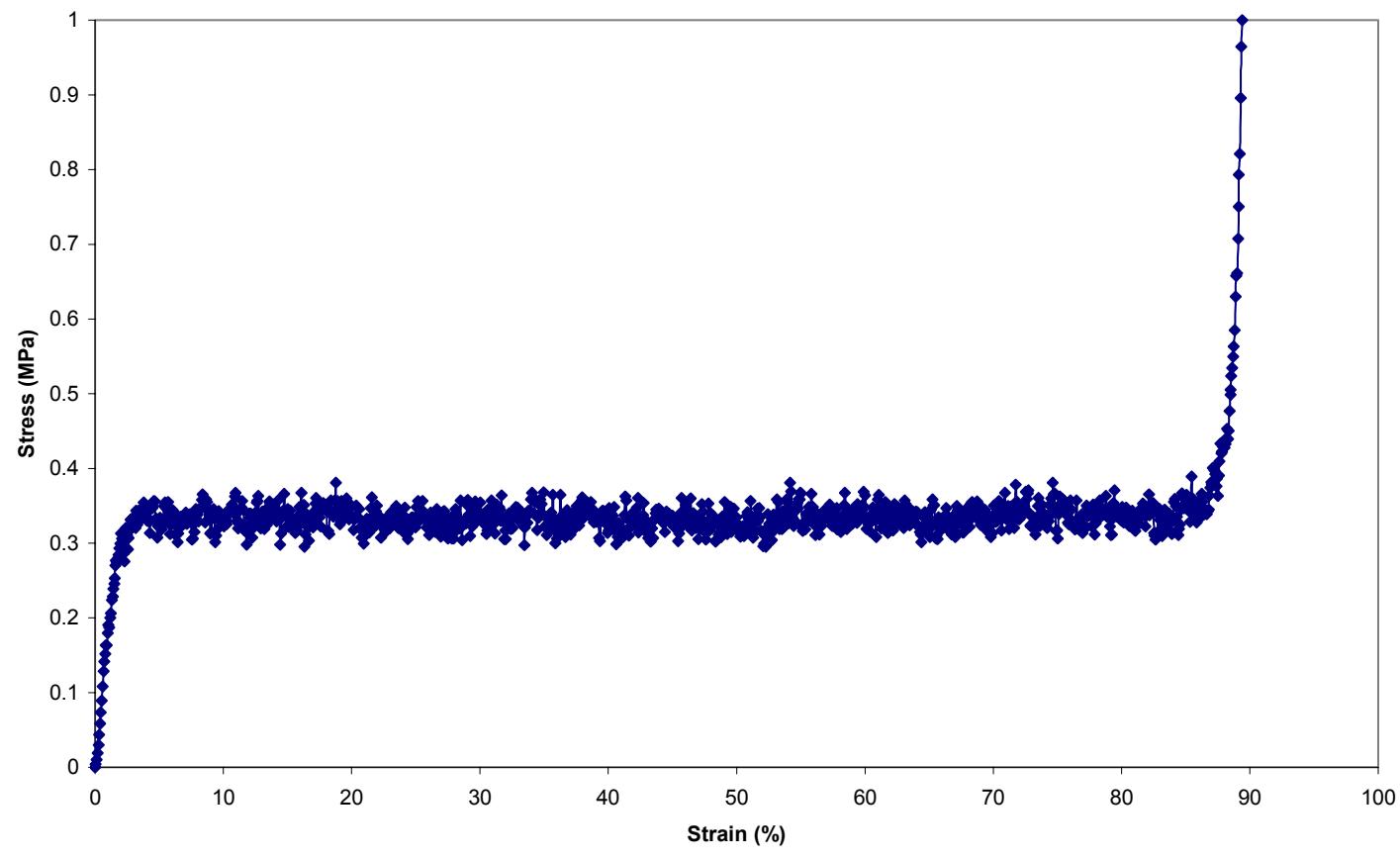

U.4.4

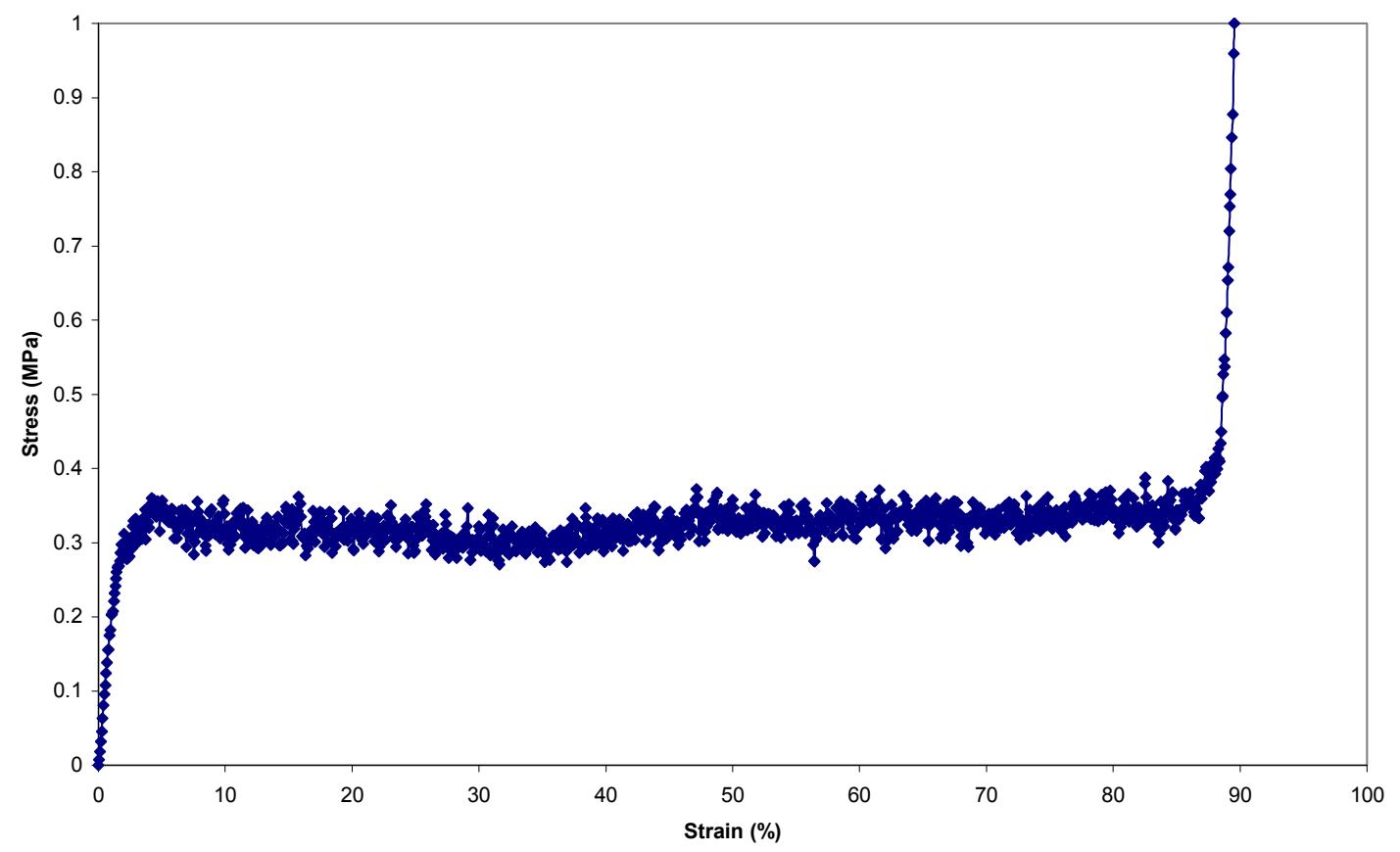

Figure A.72. Stress-Strain Plots for Samples 3 and 4 of Test U.4. 